\title{
Computation with Finitely L-Presented Groups
}

\author{
DisSERTATION \\ zur Erlangung des mathematisch-naturwissenschaftlichen \\ Doktorgrades \\ "Doctor rerum naturalium" \\ der Georg-August-Universität Göttingen
}

vorgelegt von

René Hartung

aus Magdeburg

Göttingen 2012 
Referent: Prof. Dr. Laurent Bartholdi Korreferent: Prof. Dr. Thomas Schick Tag der mündlichen Prüfung: 1. Juni 2012 


\section{Table of Contents}

\begin{tabular}{lll}
\hline & Introduction & 1
\end{tabular}

2 Groups and Presentations 9

2.1 Finite Presentations . . . . . . . . . . . . . . . . . . 9

2.2 Decision Problems for Finitelv Presented Groups . . . . . . . . . . 11

2.3 Algorithms for Finitelv Presented Groups . . . . . . . . . . . . 15

2.4 Recursive Presentations . . . . . . . . . . . . . . . . . . . . . . . 21

$2.5 \quad$ Finite $L$-Presentations . . . . . . . . . . . . . . . . . . . . . . 22

2.6 Examples of Finitely $L$-Presented Groups … . . . . . . . . . 25

2.7 Decision Problems for Finitelv $L$-Presented Groups. . . . . . . . . . . 28

2.8 First Results for Finitelv $L$-Presented Groups . . . . . . . . . . . . . . 31

2.9 Algorithms for Finitely $L$-Presented Groups . . . . . . . . . . . . 32

3 The Group Homomorphism Problem 35

3.1 The Class of Finite Grouns . . . . . . . . . . . . . . . . . . . 35

3.2 The Class of Finitelv Generated Nilpotent Groups . . . . . . . . . . . 44

3.3 Finitely Presented Images . . . . . . . . . . . . . . . . . . . 44

$4 \quad$ Tietze Transformations for $L$-presentations $\quad 47$

4.1 Tietze Transformations for finite $L$-presentations . . . . . . . . . 48

4.2 Tietze's Theorem for Finite $L$-Presentations . . . . . . . . . . . . . . 53

5 Finitelv Based Infinite Rewriting Systems 55

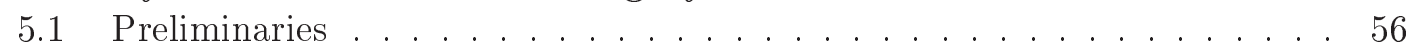

$5.2 \quad$ Monoid $L$-Presentations . . . . . . . . . . . . . . . . . . . . . . . 59

$5.3 \quad$ A Reduction Ordering on $\mathcal{S}(\mathcal{X}, \Phi) \ldots \ldots \ldots \ldots \ldots \ldots$

5.4 The Critical Pair Lemma . . . . . . . . . . . . . . . . . . 64

$5.5 \quad$ A Generalized Knuth-Bendix Completion . . . . . . . . . . . . . 68

5.6 An Application of the Knuth-Bendix Approach . . . . . . . . . . . 71

\begin{tabular}{lll}
\hline A Investigating self-similar groups & 79
\end{tabular}

A.1 Introduction . . . . . . . . . . . . . . . . . . . . . . . 79

A.2 Self-Similar Groups . . . . . . . . . . . . . . . . . . . . . . 81

A.3 A Nilpotent Quotient Algorithm . . . . . . . . . . . . . . . 83

A.4 Computing Dwyer Quotients of the Schur Multiplier . . . . . . . . . 86 
A.5 Coset Enumeration for Finite Index Subgroups . . . . . . . . . . . . . . . 88

A.6 Computing Solvable Quotients . . . . . . . . . . . . . . . . . . . . . . 91

B A Note on Invariantly Finitely $L$-Presented Groups 95

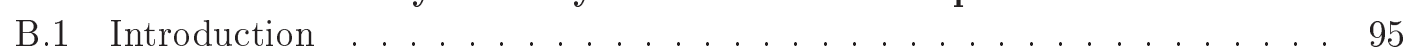

B.2 Preliminaries . . . . . . . . . . . . . . . . . . . . . . 97

B.3 Tietze Transformations for $L$-Presentations . . . . . . . . . . . . . . . . . 99

B.4 Applications of Tietze Transformations . . . . . . . . . . . . . . . . 105

B.5 Finitely generated normal subgroups of finitely presented groups . . . . . 108

C Coset Enumeration for Certain Infinitely Presented Groups 123

C.1 Introduction . . . . . . . . . . . . . . . . . . . . . . 123

C.2 Preliminaries . . . . . . . . . . . . . . . . . . . . . . . . . . 124

C.3 Coset Enumeration for Finitely $L$-Presented Groups . . . . . . . . . . . . 125

C.4 Deciding Validity of a Permutation Representation . . . . . . . . . . . . 127

C.5 Further Applications . . . . . . . . . . . . . . . . . . . . . 130

D A Reidemeister-Schreier Theorem for Finitely $L$-Presented Groups 135

D.1 Introduction . . . . . . . . . . . . . . . . . . . . . . . 135

D.2 Preliminaries . . . . . . . . . . . . . . . . . . . . . . . . . . . 137

D.3 The Reidemeister-Schreier Process . . . . . . . . . . . . . . . . . . . 141

D.4 A Trpical Example of a Subgroup $L$-Presentation . . . . . . . . . . . . 142

D.5 Stabilizing Subgroups . . . . . . . . . . . . . . . . . . . . . . 143

D.6 The Reidemeister-Schreier Theorem . . . . . . . . . . . . . . . . 150

D.7 Invariant Subgroup $L$-Presentations . . . . . . . . . . . . . . . . 153

D.8 Examples of Subgroup L-Presentations . . . . . . . . . . . . . . . . . . 158

\begin{tabular}{|ll}
\hline E Approximating the Schur Multiplier & 163
\end{tabular}

E.1 Introduction . . . . . . . . . . . . . . . . . . . . . . . 163

E.2 Preliminaries . . . . . . . . . . . . . . . . . . . . . . . . . . . 164

E.3 Adiusting an Invariant $L$-Presentation . . . . . . . . . . . . . . 166

E.4 A Generating Set for the Schur Multiplier . . . . . . . . . . . . . . . 168

E.5 Approximating the Schur Multiplier . . . . . . . . . . . . . . . . . . . 169

E.6 Applications . . . . . . . . . . . . . . . . . . . . . . . . . . 170

\begin{tabular}{ll}
\hline Bibliography & 177
\end{tabular}

\begin{tabular}{lr}
\hline Curriculum Vitæ & 187
\end{tabular} 
chaperer 1

\section{Introduction}

The Burnside problems are among the most influential problems in combinatorial group theory. The general Burnside problem asks whether a finitely generated group in which every element has finite order is finite [30]. This question was answered negatively by Golod [51]. The first explicit counter-examples to the general Burnside problem were constructed by Alešin [5], Grigorchuk [53], and Gupta \& Sidki [62]. These counterexamples can be realized as subgroups of the automorphism group of a rooted regular tree. They are the first examples of so-called 'self-similar groups'.

Self-similar groups have appeared across a wide range of mathematics, answering classical questions in infinite group theory. For instance, beside being a counter-example to the general Burnside problem, the group considered by Grigorchuk in [53] is the first example of a group with an intermediate word growth [54], answering a classical question by Milnor [100]. Moreover, this group was used in the construction of a finitely presented amenable group which is not elementary amenable [55], answering a classical question by Day [37].

The groups considered by Grigorchuk [53] and Gupta \& Sidki 62] are not finitely presented [56, 96, 128]. However, they admit recursive presentations which are nowadays known as finite L-presentations [56] or endomorphic presentations [6]. A finite $L$ presentation for Grigorchuk's group was computed by Lysënok [96]. As a homage to this work, it was suggested in [56] that a recursive presentation similar to the one computed by Lysënok [96] be called a finite $L$-presentation. This notion of a finite $L$-presentation was generalized in [6] as follows: a finite L-presentation is a group presentation of the form

$$
\left\langle\mathcal{X} \mid \mathcal{Q} \cup \bigcup_{\sigma \in \Phi^{*}} \mathcal{R}^{\sigma}\right\rangle,
$$

where $\mathcal{X}$ is a finite alphabet, $\mathcal{Q}$ and $\mathcal{R}$ are finite subsets of the free group $F$ over the alphabet $\mathcal{X}$, and $\Phi^{*}$ denotes the monoid of endomorphisms of $F$ that is generated by the finite set $\Phi \subseteq \operatorname{End}(F)$. On the one hand, this notion allows one to prove that various finitely generated self-similar groups are finitely $L$-presented but do not admit a finite presentation [6]. On the other hand, this notion is sufficiently general so that other 
infinitely presented groups are finitely $L$-presented; e.g., the groups in [18, 26, 85] admit finite $L$-presentations. Even though a finite $L$-presentation is possibly an infinite group presentation, it can be described in finite terms. Thus a finite $L$-presentation provides a finite data structure that allows one to define infinitely presented groups in a computer algebra system.

Group presentations play an important role in computational group theory. In particular finite group presentations have been the subject of intensive research during the last decades - dating back to the early days of computer algebra systems [102]. Finite $L$ presentations were recently shown to be applicable to computer algorithms as well: A first algorithm for finitely $L$-presented groups is the nilpotent quotient algorithm in [9,64. A major motivation for introducing this algorithm was the desire to understand better the striking patterns along the lower central series of various self-similar groups [7, 10, 123].

Beside using a finite $L$-presentation to study the structure of a self-similar group, its recursive action on the tree often allows one to use finite approximations of this action and thus to deal with finite quotients of the group. For various self-similar groups, including Grigorchuk's group from [53], it suffices to consider finite approximations [8] because they have the congruence property: every finite index subgroup contains the fixator of a level of the tree. However, there exist self-similar groups which do not have the congruence property and thus may not be understood via finite approximations [14. For these groups, a finite $L$-presentation is essential in studying the structure of the group.

In this thesis, we concentrate on finite $L$-presentations. More precisely, analogously to the standard reference for computing with finitely presented groups [129], it is the aim of this thesis to develop methods for studying the structure of a group for which a finite $L$-presentation is known. Because every finite presentation can be considered as a finite $L$-presentation, most algorithmic problems for finitely $L$-presented groups are not decidable - in the sense that there exists no algorithm which terminates and which returns a correct answer to a given question (e.g., whether or not a group defined by a finite $L$-presentation is finite). In special cases, though, we are able to derive algorithms for finitely $L$-presented groups. For instance, we develop a coset enumerator for finite index subgroups of a finitely $L$-presented groups in [67] or Appendix C.

The algorithms developed in this thesis can be applied by hand to obtain a solution to a given problem and they also provide explicit solutions to some decision problems. Moreover, our algorithms have been implemented in the computer algebra system GAP [50]. In Appendix A or [68, we demonstrate how detailed information on some self-similar groups could be obtained with the help of our implementations.

Parts of this thesis have appeared in [66, 67, 70]. These papers are attached in the Appendices $\mathrm{C} \mathrm{E}$. Moreover, few parts of Appendix $\mathrm{A}$ were intended to be published in [42]. 


\section{An Overview of this Thesis}

In the following, we give an overview of this thesis and its appendices. In Chapter 2, we summarize the well-known results for finitely presented groups and we formally introduce the notion of a finite $L$-presentation. Moreover, Chapter2 contains first results for finitely $L$-presented groups as, for instance, obtained in [6, 9, 64].

We then address, in Chapter 3, a first algorithmic problem for finite $L$-presentations, the so-called group homomorphism problem: In general, a group presentation is useful to decide whether or not a map from the generators of the group into another group extends to a group homomorphism; namely, if and only if all relations are mapped trivially. The group homomorphism problem for a class $\mathcal{H}$ of groups asks for an algorithm that decides whether or not such a map extends to a group homomorphism. For finite presentations, the group homomorphism problem is (semi-)decidable whenever the groups in the class $\mathcal{H}$ have a (semi-)decidable word problem. For finitely $L$-presented groups, though, there are possibly infinitely many relations which need to be considered. In Chapter 3, we give an explicit solution to the group homomorphism problem for the class of finite groups. Our solution allows us to develop various algorithms for finitely $L$-presented groups. For instance, the coset enumerator in [67] or Appendix C is a consequence of our solution to the group homomorphism problem for the class of finite groups.

In Chapter 4, we generalize Tietze's theorem for finite presentations [132 to finite $L$-presentations. This theorem and the transformations introduced in its proof allow one to modify a finite $L$-presentation without changing the isomorphism type of the group.

Finally, in Chapter [5, we generalize the Knuth-Bendix completion for finite rewriting systems to possibly infinite rewriting systems, the so-called finitely based infinite rewriting systems. These rewriting systems are derived from a finite $L$-presentation of a group. An implementation of our Knuth-Bendix completion for finitely based infinite rewriting systems does not terminate within a reasonable amount of time for Grigorchuk's group [53], for the Basilica group [61], or even for the wreath products $\mathbb{Z} \imath \mathbb{Z}$ and $\mathbb{Z}_{2} \imath \mathbb{Z}$. Therefore, our generalized Knuth-Bendix completion does not allow us to solve the word problem of the groups using their finite $L$-presentations.

The appendices to this thesis could be summarized as follows:

\section{Investigating the Schur Multiplier of a Finitely $L$-Presented Group}

It was shown in [56] that the group $\mathfrak{G}$ considered by Grigorchuk in [53] is not finitely presented. The strategy in this proof is to consider the Schur multiplier of the group. It is well-known that the Schur multiplier is a finitely generated abelian group whenever the group admits a finite presentation. The group $\mathfrak{G}$ is infinitely presented because its Schur multiplier is infinitely generated, 2-elementary abelian [56]. The explicit calculations in [56] use the finite $L$-presentation from [96. Moreover, it was shown in [56] that the set of relations in [96] is minimal in the sense that no proper subset of relations is sufficient to define the group.

The Schur multiplier of a finitely presented group is not computable in general [52]. 
Since every finitely presented group admits a finite $L$-presentation, the Schur multiplier of a finitely $L$-presented group is not computable either. Even though most algorithmic problems are not decidable in general, there exist various algorithms that allow one to gain insight into the structure of a finitely presented group. Among the most important algorithms are quotient methods that compute factor groups of a finitely presented group and thus allows one to gain insight into the structure of the group. Our generalization of the nilpotent quotient algorithm [9,64] in [66] or Appendix 国follows the general idea of a quotient method: Since it cannot compute the Schur multiplier of a finitely $L$-presented group in general, it attempts to gain insight into its structure by computing successive Dwyer quotients. These quotients of the Schur multiplier often exhibit a periodic growth in their ranks. This could be considered as computational evidence that the self-similar groups considered in [6, 9, 66] have an infinitely generated Schur multiplier and thus, are infinitely presented. However, there are other successful attempts to compute the Schur multiplier of a self-similar group [14]. These calculations possibly generalize to an algorithm for other self-similar groups.

\section{A Reidemeister-Schreier Theorem for Finite $L$-Presentations}

For finitely presented groups, it is a well-known result by Reidemeister and Schreier [119, 124 that every finite index subgroup of a finitely presented group is finitely presented. The Reidemeister-Schreier process is an algorithm that allows one to compute a finite presentation for finite index subgroups of a finitely presented group. In [70] or Appendix D, we have generalized the Reidemeister-Schreier theorem and the Reidemeister-Schreier process for finitely presented groups to finitely $L$-presented groups. More precisely, we have proved that every finite index subgroup of a finitely $L$-presented group is finitely $L$ presented. Moreover, we studied conditions on a finite-index subgroup of an invariantly finitely $L$-presented group to be invariantly finitely $L$-presented.

\section{Computing with Finite Index Subgroups}

Another direction of this thesis is the coset enumerator for finitely $L$-presented groups in 67] or Appendix C. This generalized coset enumerator has various interesting applications: For instance, it shows that the generalized word problem for finite index subgroups of a finitely $L$-presented group is decidable. Moreover, our coset enumerator allows one to compute all subgroups of a finitely $L$-presented group up to a given (moderate) index. This is of particular interest because the lattice of finite index subgroups of most selfsimilar groups is widely unknown [58; e.g., even though Grigorchuk's group has been investigated for decades, little is known on the lattice of finite-index subgroups: There are seven subgroups of index two [58] which are the only maximal subgroups [116]. Moreover, there exists a method to compute all normal subgroups of Grigorchuk's group [7, 11,33]. However, its general subgroup growth is still unknown [58]. Our coset enumerator allows us to compute the number of low-index subgroups of Grigorchuk's group. There were already attempts in [11,12] which tried to compute the number of low-index subgroups. Our coset enumeration is a first, automatic computer algorithm that allows us to compute 
the correct subgroup counts in [67] or Appendix C.

\section{A Note on Invariant Finite $\boldsymbol{L}$-Presentations}

In Appendix B or [69], we introduce Tietze transformations for (possibly infinite) $L$ presentations. These transformations allow us to generalize Tietze's theorem for finite presentations [132] to invariant finite $L$-presentations. More precisely, we show that two invariant finite $L$-presentations define isomorphic groups if and only if it is possible to pass from one invariant $L$-presentation to the other by a finite sequence of Tietze transformations. The intermediate $L$-presentations in our proof are always invariant $L$-presentations.

Moreover, in Appendix B or [69], we use Tietze transformations for finite $L$-presentations to prove that 'being invariantly finitely $L$-presented' is an abstract property of a group that does not depend on the generating set. This latter result generalizes a wellknown result for finitely presented groups: if a group admits a finite presentation with respect to one generating set, then so it does with respect to any other finite generating set [38, Chapter V].

Finally, we consider finitely generated normal subgroups of finitely presented groups. We show that every finitely generated normal subgroup $H$ of a finitely presented group $G$ is invariantly finitely $L$-presented whenever $G$ splits over $H$. This generalizes a recent result by Benli on indicable groups [22]. In fact, Benli proved that every finitely generated subgroup $H \unlhd G$ is invariantly finitely $L$-presented whenever $G / H \cong \mathbb{Z}$ holds. We also extend this latter result to the case where $G / H$ is a finitely generated abelian group with torsion-free rank at most two. Our results address a question in [22] on finitely generated subgroups which embed as normal subgroups into finitely presented groups. More precisely, analogously to Higman's embedding theorem 81, Benli asked whether or not a finitely generated group embeds as a normal subgroup into a finitely presented group if and only if the group admits an invariant finite $L$-presentation all of whose endomorphisms induce automorphisms of the group; see Appendix B or [69] for definitions. It is immanent that every such finitely $L$-presented group embeds as a normal subgroup into a finitely presented groups. However, the converse still remains open.

\section{A Note on our Implementations}

Most of our algorithms for finitely $L$-presented groups have been implemented in the computer algebra system GAP [50]. In Appendix A or [68], we demonstrate how these implementations yield detailed information on the structure of some self-similar groups, the so-called Fabrykowski-Gupta groups. In particular, we obtain new information on their lower central series, on their Schur multiplier, on their low-index subgroups, as well as new information on their derived series.

Minor parts of Appendix \$ were intended to be published in [42]. This latter manuscript also contains a method for gaining insight into the structure of the outer automorphism group of a finitely $L$-presented group: Since the lower central series subgroups 
are characteristic, this approach considers the outer automorphisms of the nilpotent quotients of the $L$-presented group. It is a straightforward application of the nilpotent quotient algorithm in [9,64.

\section{An Overview of our Algorithms}

The algorithms for finitely $L$-presented groups that were developed in this thesis could be found in the following parts: A finite $L$-presentation of a group allows one to

- decide whether or not a map from the generators of a finitely $L$-presented group into a finite permutation group (or into a finitely generated nilpotent group) extends to a group homomorphism; see Chapter 3 ,

- compute a permutation representation for the action of a finitely $L$-presented group on the cosets of a finite index subgroup (coset enumeration); see [67] or Appendix C.

- compute the index of a subgroup, which is given by its finitely many generators, of a finitely $L$-presented group provided that this index is finite; see [67] or Appendix C.

- solve the generalized word problem for finite index subgroups of a finitely $L$ presented group; see [67] or Appendix C]

- compute the intersection of two finite index subgroups of a finitely $L$-presented group or to compute the normal core of a finite index subgroup of a finitely $L$ presented group; see Chapter 3 .

- compute all subgroups of a finitely $L$-presented group up to a given (moderate) index (low-index subgroup algorithm); see [67] or Appendix [C.

- compute a finite $L$-presentation for a finite index subgroup of a finitely $L$-presented group; see [70] or Appendix D,

- compute the sections $G^{(i)} / G^{(i+1)}$ of the derived series of a finitely $L$-presented group $G$ provided that $G / G^{(i)}$ is finite; see Chapter 3 or [68].

- modify a finite $L$-presentation of a group using Tietze transformations for finitely $L$-presented groups; see Chapter 4, Appendix B, or 69.

- compute the Dwyer quotients of the Schur multiplier of an invariantly finitely $L$ presented group; see [66] or Appendix [E.

For investigating self-similar groups with the help of our algorithms, it would be interesting if the methods in [6] could be transformed into a procedure that, given a self-similar group by its recursive action on a regular rooted tree, computes a finite $L$-presentation for the group. We are not aware of an automatic procedure which could solve this problem.

\section{Acknowledgments}

I would like to express my deepest gratitude to Laurent Bartholdi for supervising my dissertation and for providing an outstanding model of how to perform excellent research. His tremendous support during the last years made this project possible. Thank you for 
the opportunity to come to Göttingen and for your invaluable advice and help during all stages of my dissertation.

I am also grateful to Thomas Schick who accepted to be my co-advisor. His true interest in the topic was a great encouragement.

Many thanks go to Michele D'Adderio who introduced me to the (great!) Italian way of life, to Dzmitry 'Dima' Dudko, whose patience helped a lot to enlighten the 'true mathematics' behind drawing pictures in complex dynamics, and to Olivier Siegenthaler. The discussions I had with Olivier suggested some of the problems considered in this thesis.

I am also grateful to the folks who bore my presence in the same room during the last fews years: Bodgan Nica, Mahesh Sunkula, Jehad Al Jaraden, Colin Reid, Tatyana Levitina, and Hossein Narmin.

Thanks to my friends and my family for their enduring support. In particular, I am deeply indebted to Doreen. Her enduring encouragement during the last years made this project possible.

Göttingen, May 2012

René Hartung. 


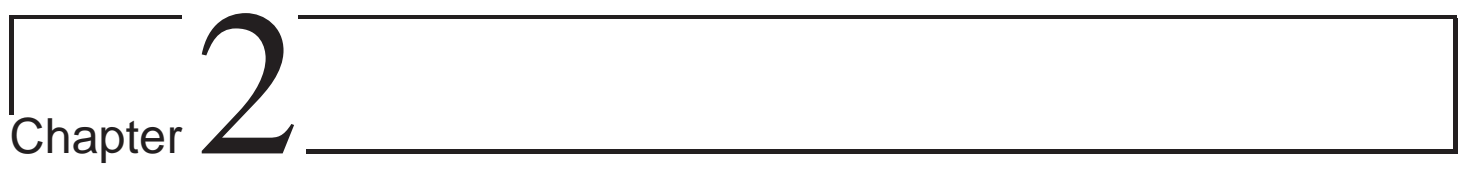

\section{Groups and Presentations}

The notion of a group presentation dates back to Walter von Dyck who has introduced the modern notion of free groups and group presentations in 1882. Group presentations play an important role in computational group theory. They define a group by its generators and relations. A finite presentation is group presentation with finitely many generators and finitely many relations. Therefore, finite presentations provide an effective definition of a group up to isomorphism. They can be used to define a group in computer algebra systems such as GAP [50] or MAGMA [28]. Recently it was shown that even a class of infinite presentations (so-called finite $L$-presentations) provide an effective way to define infinitely presented groups.

Even though finite presentations provide an effective description of a group, various algorithmic problems are unsolvable in general. Therefore, solving algorithmic problems for infinite presentations seems even more infeasible. However, in this thesis we show that most algorithms for finitely presented groups generalize to finite $L$-presentations.

In this chapter, we recall the notion of a group presentation and we formally introduce the notion of a finite $L$-presentation from [6]. Moreover, we give an overview of the algorithms known for finitely presented and finitely $L$-presented groups.

\subsection{Finite Presentations}

In the following, we recall the notion of a (finite) group presentation. For further details on finitely presented groups, we refer to [95, 97.

A group $F$ is a free group over the alphabet $\mathcal{X} \subseteq F$ if each map $f: \mathcal{X} \rightarrow G$ into a group $G$ extends to a (unique) homomorphism $\pi: F \rightarrow G$. If $G$ is generated by $\mathcal{X} \subseteq G$, the existence of a free group $F$ over the alphabet $\mathcal{X}$ shows that $G$ is a homomorphic image of $F$. In particular, if $\pi: F \rightarrow G$ denotes the natural homomorphism, $G \cong F / \operatorname{ker}(\pi)$ holds. A group presentation defines the group $G$ in terms of the free presentation $\pi: F \rightarrow G$ : Let $\mathcal{R} \subseteq F$ be a set of normal generators of the $\operatorname{kernel} \operatorname{ker}(\pi)$; i.e., the normal subgroup $\operatorname{ker}(\pi)$ is generated by $\left\{r^{g} \mid r \in \mathcal{R}, g \in F\right\}$. Then the pair $\langle\mathcal{X} \mid \mathcal{R}\rangle$ is a group presentation 
for $G$ and the group $G$ is presented by $\langle\mathcal{X} \mid \mathcal{R}\rangle$. On the other hand, a group presentation $\langle\mathcal{X} \mid \mathcal{R}\rangle$ is an abstract object that defines a group up to isomorphism: Denote by $F$ the free group over the alphabet $\mathcal{X}$ and let $K=\langle\mathcal{R}\rangle^{F}$ be the normal closure in $F$ of the relations $\mathcal{R}$; i.e., $K=\langle\mathcal{R}\rangle^{F}$ is the smallest normal subgroup of $F$ that contains $\mathcal{R}$. Then the presentation $\langle\mathcal{X} \mid \mathcal{R}\rangle$ defines the group $G=F / K$. We will also write $G=\langle\mathcal{X} \mid \mathcal{R}\rangle$ in this case.

A group presentation $\langle\mathcal{X} \mid \mathcal{R}\rangle$ is finitely generated if the set of generators $\mathcal{X}$ is finite. It is finitely related if the set of relators $\mathcal{R}$ is finite. If $\langle\mathcal{X} \mid \mathcal{R}\rangle$ is both, finitely generated and finitely related, it is a finite presentation and the group $G=\langle\mathcal{X} \mid \mathcal{R}\rangle$ it defines is finitely presented. A group that admits a finite presentation is finitely presentable (or finitely presented). If either $\mathcal{X}$ or $\mathcal{R}$ is infinite, the presentation $\langle\mathcal{X} \mid \mathcal{R}\rangle$ is an infinite presentation and the group it defines is infinitely presented by $\langle\mathcal{X} \mid \mathcal{R}\rangle$.

Being finitely presentable does not depend on a chosen generating set of the group [38, Chapter V] in the following sense: If $\langle\mathcal{X} \mid \mathcal{S}\rangle$ is an arbitrary (possibly infinite) presentation of a finitely presentable group $G$, there exists a finite subset $\mathcal{X}^{\prime} \subseteq \mathcal{X}$ and a finite set $\mathcal{S}^{\prime}$ of reduced words over $\mathcal{X}^{\prime}$ so that $G \cong\left\langle\mathcal{X}^{\prime} \mid \mathcal{S}^{\prime}\right\rangle$ holds. This can be seen as a consequence of a fundamental theorem by Tietze 132 which relates possibly different group presentations of the same group by the following Tietze transformations: Suppose that $G$ is presented by $\langle\mathcal{X} \mid \mathcal{R}\rangle$. Denote by $F$ the free group over $\mathcal{X}$ and let $K=\langle\mathcal{R}\rangle^{F}$ be given. Then $G=F / K$. Each element $r \in K$ is a consequence of the relations in $\mathcal{R}$. The following well-known transformations allow one to modify the group presentation $\langle\mathcal{X} \mid \mathcal{R}\rangle$ :

(i) If $r \in K$ is a consequence, the group $G$ is presented by $\langle\mathcal{X} \mid \mathcal{R} \cup\{r\}\rangle$.

(ii) If $z \notin \mathcal{X}$ and $w \in F$, the group presented by $\left\langle\mathcal{X} \cup\{z\} \mid \mathcal{R} \cup\left\{z^{-1} w\right\}\right\rangle$ is isomorphic to $G$.

These transformations are reversible with the following inverse transformations:

(iii) If $K=\langle\mathcal{R} \backslash\{r\}\rangle^{F}$ holds, the group $G$ is presented by $\langle\mathcal{X} \mid \mathcal{R} \backslash\{r\}\rangle$.

(iv) If $z^{-1} w \in \mathcal{R}$, with $z \in \mathcal{X}$, and $z$ does not appear in the reduced word $w, G$ is isomorphic to the group presented by $\langle\mathcal{X} \backslash\{z\} \mid \mathcal{S}\rangle$ where $\mathcal{S}$ is obtained from $\mathcal{R} \backslash\left\{z^{-1} w\right\}$ by replacing each occurrence of $z$ and $z^{-1}$ by $w$ and $w^{-1}$, respectively.

For finite presentations, these transformations are sufficient to obtain Tietze's theorem for finitely presented groups:

Theorem 2.1 (Tietze [132]) Two finite presentations define isomorphic groups if and only if it is possibly to pass for one presentation to the other by a finite sequence of Tietze transformations.

By the Nielsen-Schreier theorem [121, 6.1.1], a finite index subgroup of a finitely generated free group is finitely generated. Therefore, if $G=F / K$ is a finite group, the kernel $K$ of the free presentation $F \rightarrow G$ for a finitely generated free group $F$ is finitely generated. Thus every finite group is finitely presentable. Further examples of finitely 
presented groups are the infinite dihedral group $\left\langle\{a, b\} \mid\left\{a^{2}, b^{2}\right\}\right\rangle$, Baumslag-Solitar groups $B(m, n)=\left\langle\{a, b\} \mid\left\{b^{-1} a^{m} b a^{-n}\right\}\right\rangle$, with $n, m \in \mathbb{N}$, from [21], polycyclic groups, etc. More examples naturally arise with the following

Theorem 2.2 (Reidemeister-Schreier Theorem [119,124]) Each finite index subgroup of a finitely presented group is finitely presented.

Even though there are numerous finitely presented groups, most finitely generated groups are not finitely presented:

Proposition 2.3 (B. H. Neumann [103]) There are uncountably many non-isomorphic groups generated by two elements.

As there are countably many finite presentations but uncountably many non-isomorphic 2 -generated groups, Proposition 2.3 yields

Corollary 2.4 There are finitely generated groups that are not finitely presentable.

\subsection{Decision Problems for Finitely Presented Groups}

A finitely presented group is uniquely described by its finitely many generators and defining relations. Therefore, a finite presentation could be useful to apply computer algorithms in the investigation of the structure of the group. However, there are various problems for which it is known that there exists no algorithm that, given a finite presentation of a group, terminates and returns a correct answer to a given question (e.g. whether or not a group given by a finite presentation is finite). More precisely, we call an abstract property (i.e., a property is an abstract property of a group if it does not depend on the presentation) of a finitely presented group recursively recognizable or decidable if there exists an algorithm which takes as input a finite presentation of the group and decides whether or not the group satisfies this property.

Many abstract properties of finitely presented groups are not decidable in general. For instance, Dehn [39] stated the following decision problems: the word problem, the conjugacy problem, and the isomorphism problem. These problems are not decidable in general [1,27, 112, 113]. However, if the class of groups under consideration is restricted, decision problems that are not decidable in general may become decidable; e.g., polycyclic groups have a solvable word- and conjugacy problem 125] and even their isomorphism problem is solvable [126].

Beside Dehn's decision problems there are various other abstract properties that are not decidable. In this section, we give a brief survey on decision problems for finitely presented groups. These unsolvable problems demonstrate the limitations of computer algorithms in the study of finitely presented groups. For further details on decision problems, we refer to the survey [99] or to [95, Chapter IV]. 


\subsubsection{The Word Problem}

A first decision problem is Dehn's word problem for a finitely presented group. It asks for an algorithm that, given a finite presentation of a group $G=\langle\mathcal{X} \mid \mathcal{R}\rangle$ and a word $w$ over the alphabet $\mathcal{X} \cup \mathcal{X}^{-}$, decides whether or not the element $w$ is trivial in the group $G$. More precisely, if $F$ denotes the free group over $\mathcal{X}$ and $K \unlhd F$ is a normal subgroup so that $G=F / K$ holds, the word problem asks for an algorithm that decides whether or not $w \in K$ holds (in this case, we will also write $w=_{G} 1$ ). An element $w \in F$ is contained in the normal closure $K$ if and only if it has the form

$$
w=u_{1}^{-1} r_{1}^{\varepsilon_{1}} u_{1} \cdots u_{m}^{-1} r_{m}^{\varepsilon_{m}} u_{m}, \quad \text { with } u_{i} \in F, r_{i} \in \mathcal{R} \text { and } \varepsilon_{i}= \pm 1,
$$

for each $1 \leq i \leq m$. Clearly, if the word problem of a finitely presented group is decidable, so it is for each of its subgroups. If a group has a solvable word problem with respect to one finite presentation, then so it has for any other finite presentation [99, Lemma 2.2]. The word problem of a finitely presented group is not decidable in general:

Theorem 2.5 (Novikov-Boone Theorem [27, 113]) There exists a finitely presented group whose word problem is not decidable.

The word problem could be stated equivalently as follows: it asks for an algorithm that decides whether or not two words $w$ and $v$ over the alphabet $\mathcal{X} \cup \mathcal{X}^{-}$define the same element of the finitely presented group; i.e. if $w={ }_{G} v$ holds. Even though the word problem is not decidable in general, restricting the class of finitely presented groups may result in a class of groups with a solvable word problem. For instance each finite, free, or polycyclic group is finitely presented and has a decidable word problem.

\subsubsection{The Conjugacy Problem}

The conjugacy problem asks for an algorithm that, given a finite presentation $\langle\mathcal{X} \mid \mathcal{R}\rangle$ of a group $G$ and elements $u, v \in F$ of the free group $F$ over $\mathcal{X}$, decides whether or not the elements are conjugate in the group; i.e., if there exists $x \in F$ so that the elements $u^{x}=x^{-1} u x$ and $v$ define the same element of the group $G$; i.e. if $x^{-1} u x={ }_{G} v$ holds. Since a conjugate $x^{-1} u x$ is trivial in the group $G=\langle\mathcal{X} \mid \mathcal{R}\rangle$ if and only if the element $u$ itself is trivial, a solution to the conjugacy problem implies a solution to the word problem. Therefore, Theorem 2.5 also shows that the conjugacy problem is not decidable [112]. Again, restricting to the class of finite groups, free groups or polycyclic groups [125], yields that the conjugacy problem becomes decidable.

\subsubsection{The Generalized Word Problem}

It is easy to see that a finitely presented group has decidable word problem if there exists a finite index subgroup that has a decidable word problem. Conversely, each subgroup of a finitely presented group with a decidable word problem has a decidable word problem. Therefore, finite index subgroups play an important role in the investigation of a finitely presented group. For instance, by the Reidemeister-Schreier Theorem 2.2, each finite 
index subgroup of a finitely presented group is itself finitely presented. An explicit proof of Theorem 2.2 yields a Reidemeister-Schreier process that computes a finite presentation for the subgroup given a finite presentation of the group and a finite generating set of the subgroup [95].

A decision problem that is related to finitely generated subgroups of a finitely presented group is the generalized word problem or subgroup membership problem. It asks for an algorithm that, given a finite presentation $\langle\mathcal{X} \mid \mathcal{R}\rangle$ of a group and a finite set $\left\{w_{1}, \ldots, w_{n}, w\right\}$ of words over the alphabet $\mathcal{X} \cup \mathcal{X}^{-}$decides whether or not the subgroup generated by $\left\{w_{1}, \ldots, w_{n}\right\}$ contains the element $w$. Since an element $w \in F$ is trivial in the finitely presented group $G=\langle\mathcal{X} \mid \mathcal{R}\rangle$ if and only if it is contained in the trivial subgroup $\{1\} \leq G$, a solution to the generalized word problem implies a solution to the word problem. Hence, Theorem 2.5 also shows that the generalized word problem is not decidable in general. However, if the subgroup has finite index, coset enumeration (as outlined in Section 2.3 .1 below) solves the generalized word problem.

\subsubsection{The Isomorphism Problem}

Dehn's third decision problem is the isomorphism problem. It asks for an algorithm that decides whether or not two finite presentations define isomorphic groups. The isomorphism problem is not decidable in general [1].

Recall that Tietze's Theorem 2.1 relates two finite presentations of a group: it shows that two finite presentations define isomorphic groups if and only if there exists a finite sequence of Tietze transformations that allows one to transform one presentation into the other. However, it should be emphasized that Tietze's theorem does not provide a solution to the isomorphism problem in general, as there is no effective procedure for choosing the sequence of Tietze transformations. In particular, it does not allow to decide if two finite presentations define non-isomorphic groups; even though an enumeration of Tietze transformations may allow to eventually prove that two finite presentations define isomorphic groups 95 .

\subsubsection{Markov Properties}

Beside Dehn's decision problems many other abstract properties are not decidable. A large class of decision problems are the so-called Markov properties; see, for instance, the survey [99]. An abstract property of a finitely presented group is a Markov property if there exist finitely presented groups $G$ and $H$ so that $G$ has the given property and, if $H$ embeds into a finitely presented group, this group cannot have this property. For instance, being finite is a Markov property because the infinite dihedral group is finitely presented by $\left\langle\{a, b\} \mid\left\{a^{2}, b^{2}\right\}\right\rangle$ and infinite while the 2-elementary abelian group $\mathbb{Z}_{2} \times \mathbb{Z}_{2}=$ $\left\langle\{a, b\} \mid\left\{a^{2}, b^{2},[a, b]\right\}\right\rangle$ is finite. Further examples of Markov properties are being trivial, abelian, nilpotent, solvable, free, and torsion-free [99]. An example of an abstract property that is not a Markov property is being perfect as there exists a finitely presented perfect group in which every finitely presented group embeds [99]. Moreover, there exists an algorithm that decides whether or not a finitely presented group is perfect; see 
Section 2.3.5. A fundamental theorem for Markov properties is the following theorem:

Theorem 2.6 (Adian-Rabin Theorem [2, 3, 118]) A Markov property of a finitely presented group is not decidable.

In particular, there exists no algorithm that will compute the order of a finitely presented group.

\subsubsection{Further Decision Problems}

Beside Dehn's decision problems and Markov properties, there are other group theoretic properties that are not decidable in general.

Recall that the deficiency of a finite presentation $\langle\mathcal{X} \mid \mathcal{R}\rangle$ is $d(\langle\mathcal{X} \mid \mathcal{R}\rangle)=|\mathcal{X}|-|\mathcal{R}|$. For a finitely presented group $G$, the deficiency of the group $G$ is the maximal deficiency of all its finite presentations. The deficiency of a finitely presented groups is not computable:

Theorem 2.7 (Gordon [52]) There is no algorithm for computing the deficiency of a group from a finite presentation.

The Schur multiplier of a group $G$ can be defined as the second homology group $H_{2}(G, \mathbb{Z})$ with integer coefficients. It is related to a group presentation $G=F / R$ by Hopf's formula:

$$
H_{2}(G, \mathbb{Z}) \cong\left(R \cap F^{\prime}\right) /[R, F] .
$$

It is not hard to see that Hopf's formula shows that each finitely presented group with deficiency zero has a trivial Schur multiplier. However, even a group with a trivial Schur multiplier might not be finitely presentable [18]. The Schur multiplier of a finitely presented groups is not computable:

Theorem 2.8 (Gordon [52]) There is no algorithm for deciding, given a finite presentation of a group $G$, whether or not $H_{2}(G, \mathbb{Z})=\{0\}$ holds.

The properties in Theorems 2.7 and Theorem 2.8 are not Markov properties [99. In particular, these results are not just consequences of the Adian-Rabin theorem in Theorem 2.6. Further decision problems for finitely presented groups are given by the following proposition; see also [99]:

Proposition 2.9 (Baumslag et al. [20]) There is a finitely presented group such that there is no algorithm to determine whether or not the subgroup generated by an arbitrary finite set of words is

(i) trivial, finite, free, locally free, cyclic, abelian, nilpotent, soluble, simple, directly decomposable, freely indecomposable, or whether it is a group with a decidable word problem.

(ii) a finitely related subgroup, a subgroup with finite index, a normal subgroup, a subgroup with finitely many conjugates.

Again, restricting the class of subgroups in Proposition 2.9 to subgroups with finite index yields the existence of various algorithms that explicitly solve the problems in Proposition [2.9, (ii). 


\subsubsection{Semi-Decidability}

We call an abstract property of a finitely presented group $G$ semi-decidable if there exists an algorithm that allows one to verify that the group satisfies the given property; e.g. if there exists an algorithm that, given a finite presentation of a group, eventually terminates and proves the group to be finite. Clearly, an abstract property that is decidable is also semi-decidable. Various decision problems that are not decidable in general are semi-decidable though. For instance, the word problem of a finitely presented groups is semi-decidable: For a finitely presented group $G=\langle\mathcal{X} \mid \mathcal{R}\rangle$ and an element $w \in F$ of the free group $F$ over $\mathcal{X}$, there exists an algorithm that enumerates the elements $u_{1}^{-1} r_{1}^{\varepsilon_{1}} u_{1} \cdots u_{m}^{-1} r_{m}^{\varepsilon_{m}} u_{m}$ with $r_{i} \in \mathcal{R}, u_{i} \in F$, and $\varepsilon_{i}= \pm 1$. Therefore, the elements of the normal closure $K=\langle\mathcal{R}\rangle^{F}$ could be listed algorithmically one after another. If an element $w \in F$ is trivial in the group $G$, this algorithm would eventually list the element $w$ and therefore it would prove that $w={ }_{G} 1$ holds. Hence, the word problem of a finitely presented group is semi-decidable but not decidable.

Further examples of semi-decidable problems are the conjugacy problem and the isomorphism problem (an enumeration of Tietze transformations allows one to eventually prove that two finite presentations define isomorphic groups). Further applications of Tietze's theorem provide algorithms that allow one to prove that a finite presentation defines a trivial, abelian, nilpotent, or polycyclic group.

\subsection{Algorithms for Finitely Presented Groups}

Even though most group theoretic properties are not recursively recognizable [4], there are various 'algorithms' for finitely presented groups whose termination is often not guaranteed. If the underlying decision problem is semi-decidable, though, the algorithm terminates and gives a correct answer to the problem; e.g. it is semi-decidable if a finite presentation defines a finite group and coset enumeration would allow to verify this; see Section 2.3.1. In this section, we give an overview of the algorithms for finitely presented groups. Even though termination of these algorithms is often not guaranteed, they have become a useful tool to investigate the structure of a finitely presented group. Most of these algorithms are available in computer algebra systems such as GAP [50] or Magma [28] or even as stand-alone C-packages such as the QuOTPIC-package [83] or the ACE-package [75]. For further details on algorithms for finitely presented groups, we refer to the standard reference [129].

\subsubsection{Coset Enumeration}

A first algorithm for finitely presented groups is the coset enumeration process introduced by Todd and Coxeter [36,133. This process attempts to enumerate the cosets of a finitely generated subgroup in a finitely presented group. If the index of the subgroup is finite, coset enumeration terminates and it computes the index of the subgroup together with a permutation representation for the group's action on the cosets. In particular, coset enumeration allows one to prove that a given finite presentation defines a finite group - 
even though being finite is a Markov property! On the other hand, though, if the index of the subgroup happens to be infinite, coset enumeration will not terminate. Moreover, by Proposition 2.9, there exists no algorithm that allows one to decide whether or not a finitely generated subgroup has finite index in a finitely presented groups. In fact, there is no upper bound on the complexity of coset enumeration. Therefore, even proving a finitely presented group being trivial is computationally a challenging problem [76, 104]. Nevertheless, coset enumeration is often used for proving finiteness of certain finitely presented groups [78 80].

Because coset enumeration, if it terminates, additionally computes a permutation representation for group's action on the cosets, it also solves the generalized word problem for a finite index subgroup of a finitely presented group. Therefore, coset enumeration has become a fundamental tool for computing with finite index subgroups; e.g., it allows one to compute the intersection of two finite index subgroups as well as the normalizer and the normal core of a finite index subgroup.

Coset enumeration is usually a first step in the investigation of finitely presented groups. For instance, the Schur multiplier of a finite permutation group is computable with the methods in [82. If a finite group is given by a finite presentation, coset enumeration will compute a permutation representation for the group and thus it could be a first step for computing the Schur multiplier of a finite, finitely presented group.

Coset enumeration is among the first algorithms in group theory that have been implemented as a computer algorithm [32,88, 102,129]. It has been developed, improved, and investigated since, see [32,48], so that numerous coset enumeration techniques are currently available. For further historical details on coset enumeration, we refer to the historical notes in [129] or to the survey [102].

\subsubsection{The Low-Index Subgroup Algorithm}

A variation of coset enumeration is the low-index subgroup algorithm [40] which attempts to compute a representative for each conjugacy class of subgroups of a finitely presented group up to a given index $n \in \mathbb{N}$. The central idea uses a backtrack-search for finding all permutation representations of a finitely presented group that acts transitively on at most $n$ points. In practice, the low-index subgroup algorithm allows one to compute representatives of conjugacy classes of subgroups with index up to a few hundred. For further details on the low-index subgroup algorithm, we refer to [129, Section 5.6].

A variation of the low-index subgroup algorithm was developed in 35. This modified algorithm computes the normal subgroups of a finitely presented groups up to a given index. In practice, it allows one to compute possibly larger indices than the standard low-index subgroup algorithm. An implementation of both algorithms is available in the stand-alone C-package Lowx [41.

The low-index subgroup algorithm yields insight into the lattice of finite index subgroups of a finitely presented group top-down and therefore, it is a useful tool in the investigation of finitely presented groups. For instance, since 'being trivial' is a Markov property, there is no algorithm for deciding whether or not a finite presentation defines 
a trivial group. However, if a finite presentation does define the trivial group, coset enumeration is guaranteed to terminate and it will compute the order of group. On the other hand, there cannot exists an algorithm which proves that a given finite presentation defines a non-trivial group (otherwise we would obtain a contradiction to the Adian-Rabin Theorem (2.6). The low-index subgroup algorithm could be employed to this problem because it may help to find a subgroup with non-trivial index. If the low-index subgroup algorithm succeeds with this search, it would prove that the given finite presentation defines a non-trivial group. An alternative approach for proving that a finite presentation defines a non-trivial group is to search for non-trivial homomorphic images of the finitely presented group; see Section 2.3.5 below.

\subsubsection{The Reidemeister-Schreier Process}

The Reidemeister-Schreier Theorem 2.2 shows that each finite index subgroup of a finitely presented group is finitely presented. A constructive proof of Theorem 2.2 is given by the Reidemeister-Schreier process: it takes as input a finite presentation of a group and a finite generating set of a subgroup and it computes a finite presentation for the subgroup provided that it has a finite index in the group. By Proposition 2.9, there cannot exist such an algorithm in general (in particular if the subgroup has infinite index). Suppose that the finitely generated subgroup $U$ has finite index in the finitely presented group $G=\langle\mathcal{X} \mid \mathcal{R}\rangle$. Then the following steps yield a Reidemeister-Schreier process:

(i) Compute a permutation representation $\tau: F \rightarrow \operatorname{Sym}(U \backslash G)$ for $G$ 's action on the cosets $U \backslash G$ using a coset enumerator.

(ii) The permutation representation $\tau$ allows one to compute finitely many Schreier generators of the subgroup [124] with the Nielsen-Schreier theorem [121, 6.1.1]; i.e., a Schreier generating set is a free generating set of the full preimage of the subgroup $U \leq G$ in the free group $F$ over $\mathcal{X}$.

(iii) The permutation representation $\tau$ yields a Reidemeister rewriting that rewrites $G$ 's relations in $\mathcal{R}$ to finitely many relations of the subgroup $U$.

For further details on the Reidemeister-Schreier process, we refer to [95, Section II.4] or Section D.3 below.

The Reidemeister-Schreier process gives an explicit method to apply the algorithms for finitely presented groups to finite index subgroups of finitely presented groups. In particular, in combination with the low-index subgroup algorithm, the ReidemeisterSchreier process yields a method to gain insight into the structure of a finitely presented group [77]. For instance, it allows one to compute the quotients of the derived series provided that these quotients are finite; see, for instance, Section A.6 below. An early implementation of the Reidemeister-Schreier process can be found in [72].

\subsubsection{Tietze Transformations}

The finite presentations that were computed with the Reidemeister-Schreier process often contain redundant generators and redundant relations. Indeed, given a finitely presented 
group $G=\langle\mathcal{X} \mid \mathcal{R}\rangle$ with $n=|\mathcal{X}|$ generators and a finite index subgroup $H \leq G$ with $m=[G: H]$, the Reidemeister-Schreier process in Section 2.3.3 computes a finite presentation with $m n-m+1$ generators [121, 6.1.1]. Numerous generators and relators are often redundant. Therefore, one wishes to remove redundant generators and relations from a presentation. Here, the Tietze transformations from Section 2.1 apply.

Since the isomorphism problem is not decidable in general, applications of Tietze transformations are limited in practice. However, an implementation of a Tietze transformation program was described in [73, 120. This procedure is part of the ReidemeisterSchreier program [72] where it simplifies the obtained finite presentation.

\subsubsection{Quotient Methods}

Most algorithmic problems for finitely presented groups are not decidable [4. Therefore, it surprises that computer algorithms help in the investigation of the structure of a finitely presented group. However, there is a wide range of algorithms for computing factor groups of a finitely presented group. For instance, the abelianization of a finitely presented group (i.e., the abelianization is the isomorphism type of the largest abelian quotient $G /[G, G]$ of a group $G$ ) can be effectively computed from a finite presentation [129, Section 11.2]. In particular, there exists an algorithm that decides whether or not a finitely presented group is perfect (i.e. a group $G$ is perfect if the abelianization $G /[G, G]$ is trivial). Moreover, there exists an algorithm that solves the membership problem for the derived subgroup $G^{\prime}=[G, G]$ in a finitely presented group.

Beside the numerous variations of coset enumeration, the main tools for investigating finitely presented groups are quotient methods. In general, they compute successive quotients of a finitely presented group or, equivalently, they compute homomorphisms into computationally 'nice' groups (e.g. finite groups, finite $p$-groups, or polycyclic groups which could be handled well in computer algebra systems). These methods also allow one to decide whether or not a map from the generating set $\mathcal{X}$ of a finitely presented group $G=\langle\mathcal{X} \mid \mathcal{R}\rangle$ into a 'nice' group $H$ extends to a homomorphism $G \rightarrow H$; see Chapter 3 . In the following, we give a survey of the quotient methods for finitely presented groups:

\section{The Abelianization Algorithm}

A first quotient method is the abelianization algorithm for computing the isomorphism type of the largest abelian quotient of a finitely presented group; see [129, Chapter 11.2]. This algorithm relies on linear algebra only. More precisely, it computes the Smith normal form of an integer matrix that can be read off directly from the relations of the finite presentation. These computations are usually fast. Therefore, the abelianization algorithm is often a first tool in the investigation of a finitely presented group. For instance, the abelianization algorithm and the low-index subgroup algorithm can be used to search for finite index subgroups with infinite abelianization. This could help proving a finitely presented group being infinite. Moreover, the derived subgroup of a finitely generated group $G=\langle\mathcal{X} \mid \mathcal{R}\rangle$ has a decidable membership problem: An element $w \in F$ of the free group $F$ over $\mathcal{X}$ defines an element of the derived subgroup $G^{\prime}=[G, G]$ 
if and only if its exponent vector vanishes (i.e. the exponent vector is the image of the element $w \in F$ in the free abelian group $\left.F / F^{\prime}\right)$.

\section{The $p$-Quotient Algorithm}

For a prime $p$, the lower exponent-p series (or Frattini series) is defined recursively by $\lambda_{1} G=G$ and $\lambda_{n+1} G=\left[G, \lambda_{n} G\right]\left(\lambda_{n} G\right)^{p}$. The sections $\lambda_{n} G / \lambda_{n+1} G$ are $p$-elementary abelian so that $G / \lambda_{n} G$ are finite $p$-groups. Finite $p$-groups can be described effectively by nilpotent presentations. A nilpotent presentation is a finite presentation which admits an effective solution to the word problem of the group [129].

The p-quotient algorithm computes a nilpotent presentation for the factor group $G / \lambda_{n} G$ together with a homomorphism $G \rightarrow G / \lambda_{n} G$. It uses an induction on $n$ and it computes the quotient $G / \lambda_{n} G$ as a finite central extension of a $p$-elementary abelian group by a finite $p$-group. The $p$-quotient algorithm was originally designed for the construction of finite Burnside groups in [74, 105].

\section{The Nilpotent Quotient Algorithm}

The p-quotient algorithm was generalized in [107] to the nilpotent quotient algorithm for finitely presented groups; see also [129, Section 11]. For a finitely presented group $G$ and a positive integer $c$, the nilpotent quotient algorithm computes a nilpotent presentation for the lower central series quotient $G / \gamma_{c+1} G$ together with a homomorphism $G \rightarrow G / \gamma_{c+1} G$. The lower central series is defined recursively by $\gamma_{1} G=G$ and $\gamma_{c+1} G=\left[G, \gamma_{c} G\right]$. Similar to the $p$-quotient algorithm, the nilpotent quotient algorithm uses an induction on $n$. The induction starts with the largest abelian quotient $G / G^{\prime}$ of a finitely presented group $G$ and thus it starts with the abelianization algorithm for finitely presented groups. Then the quotients $G / \gamma_{c+1} G$ are constructed as central extension of a finitely generated abelian group by a finitely generated nilpotent group.

\section{Soluble Quotient Algorithms}

For a group $G$, the derived series is defined recursively by $G^{(1)}=G^{\prime}=[G, G]$ and $G^{(i+1)}=\left[G^{(i)}, G^{(i)}\right]$. If, for each $1 \leq i \leq j$, the sections $G^{(i)} / G^{(i+1)}$ are finitely generated, the quotient $G / G^{(j)}$ is polycyclic. A polycyclic group can be described by a polycyclic presentation that (like a nilpotent presentation) yields an effective solution to the word problem.

A soluble quotient algorithm computes a polycyclic presentation of the quotient $G / G^{(i)}$ together with a homomorphism $G \rightarrow G^{(i)}$ provided that $G / G^{(i)}$ is polycyclic. A first straightforward approach towards a soluble quotient algorithm combines the abelianization algorithm and the Reidemeister-Schreier process; see Section A.6 below. This approach computes a permutation representation for $G / G^{(i)}$ provided that this quotient is finite. Alternative approaches which compute polycyclic presentations for finite solvable quotients were described by Wamsley [135], Leedham-Green [89], Plesken [117, and Niemeyer [108 110]. These methods possibly use a different series of normal subgroups. 
A more general soluble quotient algorithm is the polycyclic quotient algorithm in [92, 93]. This algorithm computes a polycyclic presentation for $G / G^{(i)}$. Similar to the nilpotent and the $p$-quotient algorithm, it uses an induction on $i$ and it therefore computes a polycyclic presentation for $G / G^{(i+1)}$ from a polycyclic presentation of $G / G^{(i)}$. For this purpose, it uses a Gröbner bases approach which is guaranteed to terminate. Even if $G$ and $G / G^{(i)}$ are finitely generated, the section $G^{(i)} / G^{(i+1)}$ is possibly not finitely generated. In this case, $G / G^{(i+1)}$ is not polycyclic. The algorithm in [92,93] either computes a polycyclic presentation for $G / G^{(i+1)}$ or it returns fail if the section $G^{(i)} / G^{(i+1)}$ is not finitely generated. If a finite presentation defines a polycyclic group, the algorithm in 92,93 w would compute a polycyclic presentation for it.

\subsubsection{Knuth-Bendix Completion}

A practical method for solving the word problem is given by a confluent rewriting system. The Knuth-Bendix completion attempts to construct a confluent rewriting system from a monoid presentation of a finitely presented group [130]. Since the word problem of a finitely presented group is not decidable in general, termination of the Knuth-Bendix completion is not guaranteed. If the Knuth-Bendix completion terminates, though, the confluent rewriting system solves the word problem as it allows one to compute normal forms for each element of the group. For further details on rewriting systems, we refer to [129, Chapter 2] or Chapter 5 below.

A finitely presented group $G=\langle\mathcal{X} \mid \mathcal{R}\rangle$ can be considered as a finitely presented monoid

$$
\left\langle\mathcal{X} \cup \mathcal{X}^{-} \mid\{r=\varepsilon \mid r \in \mathcal{R}\} \cup\left\{x^{-1} x=x x^{-1}=\varepsilon \mid x \in \mathcal{X}\right\}\right\rangle,
$$

where $\varepsilon$ denotes the empty string. A rewriting system for a finitely presented monoid can be seen as a binary relation on the words over the alphabet $\mathcal{X} \cup \mathcal{X}^{-}$together with a reduction ordering $\prec$; i.e., a reduction ordering is a translation invariant well-ordering. From a finite presentation of a monoid one can obtain a rewriting system by defining, for each relation $A=B$ of the presentation, either the rewriting rule $A \rightarrow B$, if $B \prec A$ holds, or $B \rightarrow A$, otherwise. A reduction of a word $W \in\left(\mathcal{X} \cup \mathcal{X}^{-}\right)^{*}$ replaces a subword $A$ of $W$ by $B$ whenever $A \rightarrow B$ is a rewriting rule. As $W=U A V$ holds and $\prec$ is translation invariant, the reduction $W=U A V \rightarrow U B V$ yields that $W=U A V \succ U B V$ as $A \succ B$ holds. Thus, as the reduction ordering $\prec$ is a well-ordering, a reduction of $W$ yields a finite sequence $W=W_{1} \succ W_{2} \succ \ldots \succ W_{n}$ with $W_{i} \rightarrow W_{i+1}$.

There are possibly different choices of the next rewriting rule to apply within a reduction of a given word $W$. These choices may result into different least elements $W_{n}$. A rewriting system is called confluent, though, if, for each word $W$, the least element $W_{n}$ does not depend on the choice of the intermediate reductions. A confluent rewriting system provides a method for transforming any word over $\mathcal{X} \cup \mathcal{X}^{-}$into its unique normal form. In fact, it solves the word problem of the group. For instance, finitely generated nilpotent groups or polycyclic groups are examples of groups which admit a finite confluent rewriting system [129], so-called confluent polycyclic presentations. 
The Knuth-Bendix completion (see, for instance, [129, 130]) transforms a rewriting system for a finitely presented monoid into a confluent rewriting system. For this purpose, it checks certain overlaps of the rules and it adds rewriting rules if necessary. We outline the details of a Knuth-Bendix completion within our generalization in Chapter 5 below.

\subsection{Recursive Presentations}

In this section, we introduce the notion of a recursive presentation and the notion of a recursively presented group. For further details on recursively presented groups, we refer to Chapter II of [95].

Let $F$ be a finitely generated free group. We call a subset $S \subseteq F$ recursively enumerable if there exists an algorithm that lists the elements of $S$ in some (arbitrary) order. In this case, we can write $S=\left\{s_{1}, s_{2}, s_{3}, \ldots\right\}$ where $s_{n}$ denotes the result of the algorithm that is returned after the $n$-th step. For instance, a finite set and a finitely generated free monoid are recursively enumerable.

A subset $S \subset F$ is recursive if there exists an algorithm that decides whether or not a given element $w \in F$ belongs to the set $S$. A recursive set $S \subseteq F$ is clearly recursively enumerable because if an algorithm that lists the elements of the finitely generated free group $F$ can be combined with the algorithm that decides whether or not an element belongs to the recursive set $S$. A set $S$ is recursive if and only if $S$ and its complement $F \backslash S$ are recursively enumerable. On the other hand, it is a fundamental result in logic that there exists a recursively enumerable set that is not recursive. This is the reason for most problems in group theory not being decidable [95, 99].

A recursive presentation is a group presentation $\langle\mathcal{X} \mid \mathcal{R}\rangle$ with a finite alphabet $\mathcal{X}$ and a recursively enumerable set of relations $\mathcal{R} \subseteq F$ where $F$ denotes the free group over the alphabet $\mathcal{X}$. The group $G=\langle\mathcal{X} \mid \mathcal{R}\rangle$ defined by a recursive presentation is recursively presented. As finite sets are recursively enumerable, every finite presentation is a recursive presentation. Recursively presented groups have been classified by Higman's embedding theorem:

Theorem 2.10 (Higman's Embedding Theorem [81]) A finitely generated group embeds into a finitely presented group if and only if it is recursively presented.

This is a fundamental result in combinatorial group theory. For instance, the existence of a recursively enumerable set of integers that is not recursive is used in [95, Section IV.7] for the construction of a recursively presented group whose word problem is not decidable. As this recursively presented group embeds into a finitely presented group by Theorem 2.10, there exists a finitely presented group whose word problem is not decidable [95, Theorem IV 7.2]. This would prove the Novikov-Boone Theorem [2.5.

If a group $G$ is finitely generated by $\mathcal{X}$, it has a solvable word problem if and only if the set $\mathcal{S}=\left\{w \in F(\mathcal{X}) \mid w={ }_{G} 1\right\}$ is recursive. In particular, the word problem is decidable if and only if $\mathcal{S}$ and its complement $F \backslash \mathcal{S}$ are recursively enumerable. In this case, a recursive presentation for the group $G$ is given by $\left\langle\mathcal{X} \mid\left\{w \in F(\mathcal{X}) \mid w=_{G} 1\right\}\right\rangle$. 
Therefore, every finitely generated group with a solvable word problem is recursively presented. Hence, it embeds into a finitely presented group with Higman's Embedding Theorem 2.10, More specifically, one can prove the following

Proposition 2.11 (Clapham [34, 99]) A finitely generated group with a decidable word problem embeds into a finitely presented group whose word problem is decidable.

\section{$2.5 \quad$ Finite $L$-presentations}

In this section, we finally introduce the notion of a finite $L$-presentation and the notion of a finitely $L$-presented group. The study of finite $L$-presentations was initiated by Lysënok's result in [96] for the group $\mathfrak{G}$ constructed by Grigorchuk [53]: he proved that $\mathfrak{G}$ is infinitely presented by

$$
\mathfrak{G} \cong\left\langle\{a, b, c, d\} \mid\left\{a^{2}, b^{2}, c^{2}, d^{2}, b c d\right\} \cup \bigcup_{i \in \mathbb{N}_{0}}\left\{(a d)^{4},(a d a c a c)^{4}\right\}^{\sigma^{i}}\right\rangle,
$$

where $\sigma$ is an endomorphism of the free group over $\{a, b, c, d\}$ that is induced by the map $a \mapsto a c a, b \mapsto d, c \mapsto b$, and $d \mapsto c$. As a homage to this work and as a reference to the $L$-systems from [91], it was suggested in [56] that a group is called finitely $L$-presented if it admits a group presentation of the form

$$
\left\langle\mathcal{X} \mid \mathcal{Q} \cup \bigcup_{i \in \mathbb{N}_{0}} \mathcal{R}^{\sigma^{i}}\right\rangle
$$

with a finite alphabet $\mathcal{X}$, finite subsets $\mathcal{Q}$ and $\mathcal{R}$ of the free group over $\mathcal{X}$, and a single endomorphism $\sigma$ of the free group over $\mathcal{X}$. This notion was generalized in [6] as follows:

Definition 2.12 (Bartholdi [6]) An L-presentation is a group presentation of the form

$$
\left\langle\mathcal{X} \mid \mathcal{Q} \cup \bigcup_{\sigma \in \Phi^{*}} \mathcal{R}^{\sigma}\right\rangle
$$

where $\Phi^{*}$ denotes the free monoid of endomorphisms of the free group $F$ over $\mathcal{X}$ that is generated by $\Phi$; i.e., the closure of $\{\mathrm{id}\} \cup \Phi$ under taking composition of group homomorphisms.

The group presentation in Eq. (2.3) is denoted by $\langle\mathcal{X}|\mathcal{Q}| \Phi \mid \mathcal{R}\rangle$. The group $G$ it defines is L-presented by $\langle\mathcal{X}|\mathcal{Q}| \Phi \mid \mathcal{R}\rangle$. We also write $G=\langle\mathcal{X}|\mathcal{Q}| \Phi \mid \mathcal{R}\rangle$ in this case. An $L$-presentation $\langle\mathcal{X}|\mathcal{Q}| \Phi \mid \mathcal{R}\rangle$ is a finite L-presentation if $\mathcal{X}, \mathcal{Q}, \Phi$, and $\mathcal{R}$ are finite. A group presented by a finite $L$-presentation is finitely L-presented by $\langle\mathcal{X}|\mathcal{Q}| \Phi \mid \mathcal{R}\rangle$. The relations in $\mathcal{Q}$ and $\mathcal{R}$ are the fixed relations and iterated relations, respectively. The endomorphisms in $\Phi$ are the substitutions of the $L$-presentations. 
Remark 2.13 In the following, we always assume that the substitutions in $\Phi$ of a finite $L$-presentation generate a free monoid. This is not necessary for the algorithms constructed below and, in particular, it does not change the isomorphism type of the group. More precisely, given a finite set of endomorphisms $\left\{\sigma_{1}, \ldots, \sigma_{n}\right\} \subseteq \operatorname{End}(F)$ and a finite set of symbols $\Phi=\left\{\phi_{1}, \ldots, \phi_{n}\right\}$, we consider the free monoid $\Phi^{*}$ which naturally maps onto the monoid $M=\left\langle\sigma_{1}, \ldots, \sigma_{n}\right\rangle$ generated by the endomorphism $\left\{\sigma_{1}, \ldots, \sigma_{n}\right\}$ of the free group. In the following, we always identify the free monoid $\Phi^{*}$ with its image in $\operatorname{End}(F)$. This identification does not change the isomorphism type of the L-presented group. However, the free monoid $\Phi^{*}$ is well-ordered [67, 129] which we often use for the algorithms constructed below.

With the notion of a finite $L$-presentation, Lysënok's result in Eq. (2.2) could be restated as follows:

Theorem 2.14 (Lysënok [96]) The Grigorchuk group $\mathfrak{G}$ is finitely L-presented by $\left.\left\langle\{a, b, c, d\}\left|\left\{a^{2}, b^{2}, c^{2}, d^{2}, b c d\right\}\right|\{\sigma\}\right|\left\{(a d)^{4},(\text { adacac })^{4}\right\}\right\rangle$ where $\sigma$ denotes the endomorphism of the free group over $\{a, b, c, d\}$ that is induced by the map $a \mapsto a c a, b \mapsto d, c \mapsto b$, and $d \mapsto c$.

An $L$-presentation of the form $\langle\mathcal{X}|\emptyset| \Phi \mid \mathcal{R}\rangle$ is an ascending L-presentation. Ascending $L$-presentations generalize the concept of a finite presentation:

Remark 2.15 A finitely presented group $\langle\mathcal{X} \mid \mathcal{R}\rangle$ is finitely L-presented by $\langle\mathcal{X}|\emptyset| \emptyset \mid \mathcal{R}\rangle$.

The substitutions $\sigma \in \Phi$ of an ascending $L$-presentation $\langle\mathcal{X}|\emptyset| \Phi \mid \mathcal{R}\rangle$ stabilize the normal subgroup $K=\left\langle\bigcup_{\sigma \in \Phi^{*}} \mathcal{R}^{\sigma}\right\rangle^{F}$. Therefore, each substitution of an ascending $L$-presentation induces an endomorphism of the $L$-presented group $G=F / K$. An $L$-presentation where every substitution induces an endomorphism of the $L$-presented group is called an invariant L-presentation. The group presented by an invariant $L$ presentation is invariantly L-presented. Some algorithms developed in this thesis will work for invariant $L$-presentations only; e.g. the algorithm in Appendix E for computing Dwyer quotients of the Schur multiplier of an invariantly finitely $L$-presented group. Additionally, the nilpotent quotient algorithm in [9,64 is more effective on invariant finite $L$-presentations. Note that invariant and ascending $L$-presentations are related by the following

Remark 2.16 Every ascending L-presentation is invariant. For each invariant L-presentation $\langle\mathcal{X}|\mathcal{Q}| \Phi \mid \mathcal{R}\rangle$ there exists an ascending L-presentation $\langle\mathcal{X}|\emptyset| \Phi \mid \mathcal{Q} \cup \mathcal{R}\rangle$ which defines the same group.

The finite $L$-presentation in Theorem 2.14 for the group $\mathfrak{G}$ constructed by Grigorchuk is an invariant $L$-presentation as the fixed relations $\mathcal{Q}=\left\{a^{2}, b^{2}, c^{2}, d^{2}, b c d\right\}$ are mapped by the substitution $\sigma$ to

$$
\left(a^{2}\right)^{\sigma}=(a c a)^{2}, \quad\left(b^{2}\right)^{\sigma}=d^{2}, \quad\left(c^{2}\right)^{\sigma}=b^{2}, \quad\left(d^{2}\right)^{\sigma}=c^{2}, \text { and }(b c d)^{\sigma}=d b c=(b c d)^{d^{-1}} .
$$


These images are obviously relations in the group $\mathfrak{G}$. Therefore, the substitution $\sigma$ factors through to an endomorphism of $\mathfrak{G}$; see also [55]. In particular, $\mathfrak{G}$ is finitely $L$-presented by the ascending finite $L$-presentations

$$
\begin{aligned}
& \left.\langle\{a, b, c, d\}|\emptyset|\{\sigma\}|\left\{a^{2}, b^{2}, c^{2}, d^{2}, b c d,(a d)^{4},(\text { adacac })^{4}\right\}\right\rangle \\
= & \left.\langle\{a, b, c, d\}|\emptyset|\{\sigma\}|\left\{a^{2}, b c d,(a d)^{4},(\text { adacac })^{4}\right\}\right\rangle .
\end{aligned}
$$

Finite $L$-presentations are recursive:

Lemma 2.17 A finite L-presentation is a recursive presentation.

Proof. Let $\langle\mathcal{X}|\mathcal{Q}| \Phi \mid \mathcal{R}\rangle$ be a finite $L$-presentation. Since the finitely generated free monoid $\Phi^{*}$ is recursively enumerable, the set of relations $\mathcal{Q} \cup \bigcup_{\sigma \in \Phi^{*}} \mathcal{R}^{\sigma}$ of a finitely $L$-presented group is recursively enumerable.

Higman's embedding theorem 2.10 and Lemma 2.17 have the following immediate

Corollary 2.18 Each finitely L-presented group embeds into a finitely presented group. Each finitely generated subgroup of a finitely L-presented group is recursively presented.

Proof. Since a finitely $L$-presented group is recursively presented, a finitely $L$-presented group embeds into a finitely presented group by Higman's embedding theorem 2.10 . Thus a finitely generated subgroup of a finitely $L$-presented group embeds into a finitely presented group and hence, it is recursively presented.

In Appendix D, we generalize the Reidemeister-Schreier theorem for finitely presented groups as we prove that each finite index subgroup of finitely $L$-presented group is finitely $L$-presented itself.

Proposition 2.19 The class of finitely presented groups is properly contained in the class of finitely L-presented groups. The class of finitely L-presented groups is contained in the class of recursively presented groups. There are finitely generated groups that are not finitely L-presented.

Proof. By Remark 2.15, each finitely presented group is finitely $L$-presented. Grigorchuk's group is finitely $L$-presented by Theorem 2.14 but it is not finitely presented [56]. Therefore, the class of finitely presented groups is properly contained in the class of finitely $L$-presented groups. By Lemma 2.17 , every finitely $L$-presented group is recursively presented. Because there are only countably many finite $L$-presentations but, by Proposition 2.3. uncountably many non-isomorphic groups generated by two elements, there are finitely generated groups that are not finitely $L$-presented.

Since we are not aware of a method to prove or disprove that a finitely generated group is finitely $L$-presented (or even invariantly finitely $L$-presented), we have no answer to the following problem:

Question 2.20 Is there an explicit example of a recursively presented group that is not finitely L-presented? Is there a finitely L-presented group that is not invariantly finitely L-presented? 
Note that, in Appendix B. we prove that 'being invariantly finitely $L$-presented' is an abstract property of a group which does not depend on the generating set. Therefore, it suffices to construct a finitely $L$-presented group which does not admit an invariant $L$-presentation with respect to the same generating set.

\subsection{Examples of Finitely $L$-Presented Groups}

Because finite $L$-presentations generalize the concept of a finite presentation, there are various examples of finitely $L$-presented groups. More interesting examples of finitely $L$-presented groups are those which are not finitely presented. In this section, we show that the concept of a finite $L$-presentation is quite general so that various examples of finitely generated groups exist which are finitely $L$-presented. The algorithms developed in this thesis apply to these groups.

\subsubsection{Self-Similar Groups}

A major motivation for introducing the notion of a finite $L$-presentation in [6,56] was the desire to understand better some examples of self-similar groups. Self-similar groups have appeared across a wide range of mathematics, answering classical questions in infinite group theory as well as establishing new links to complex dynamics [6, 9].

A self-similar group is defined by its recursive action on a rooted regular tree. We consider the $d$-regular rooted tree as the free monoid over the alphabet $\mathcal{T}=\{0, \ldots, d-1\}$. Then a group $G$ that acts faithfully on the free monoid $\mathcal{T}^{*}$ is a self-similar group if for each $g \in G$ and $x \in \mathcal{T}$ there exist $h \in G$ and $y \in \mathcal{T}$ with

$$
(x w)^{g}=y w^{h} \text { for each } w \in \mathcal{T}^{*} .
$$

In order to define a self-similar group, it suffices to specify the action of its generators on the regular rooted tree. For instance, we have the following well-known examples of self-similar groups:

Example 2.21 (Grigorchuk [53]) The Grigorchuk group $\mathfrak{G}=\langle a, b, c, d\rangle$ is defined by the following action on the rooted binary tree $\mathcal{T}^{*}=\{0,1\}^{*}$ :

$$
\begin{aligned}
(0 w)^{a} & =1 w & (1 w)^{a} & =0 w \\
(0 w)^{b} & =0 w^{a} & (1 w)^{b} & =1 w^{c} \\
(0 w)^{c} & =0 w^{a} & (1 w)^{c} & =1 w^{d} \\
(0 w)^{d} & =0 w & (1 w)^{d} & =1 w^{b} .
\end{aligned}
$$

Example 2.22 (Gupta \& Sidki [62]) The Gupta-Sidki group $G=\langle a, r\rangle$ is defined by the following action on the rooted 3 -regular tree $\mathcal{T}^{*}=\{0,1,2\}^{*}$ :

$$
\begin{aligned}
(0 w)^{a} & =1 w & (0 w)^{r} & =0 w^{a} \\
(1 w)^{a} & =2 w & (1 w)^{r} & =1 w^{a^{-1}} \\
(2 w)^{a} & =0 w & (2 w)^{r} & =2 w^{r} .
\end{aligned}
$$


Example 2.23 (Fabrykowski \& Gupta [45, 46], Grigorchuk [57]) For $d \geq 3$, the generalized Fabrykowski-Gupta group $\Gamma_{d}=\langle a, r\rangle$ is defined by the following action on the rooted d-regular tree $\mathcal{T}^{*}=\{0, \ldots, d-1\}$ :

$$
\begin{aligned}
& (x w)^{a}=x+1 \quad(\bmod d) w, \quad \text { for } 0 \leq x \leq d-1 \\
& (0 w)^{r}=0 w^{a} \\
& (x w)^{r}=x w, \quad \text { for } 1 \leq x<d-1 \\
& (d-1 w)^{r}=d-1 w^{r} .
\end{aligned}
$$

Various self-similar groups are finitely $L$-presented but not finitely presented:

Theorem 2.24 (Bartholdi [6]) Each finitely generated, contracting, semi-fractal, regular branch group is finitely L-presented but not finitely presented.

The constructive proof of Theorem 2.24 provides a method for computing finite $L$ presentations for self-similar groups; see [6, 14, 128]. For further details on self-similar groups groups, we refer to [6, 12, 101]. Throughout this thesis, we often use the testbedgroups from [6]9] to provide computational evidence for the effectiveness of our algorithms in practice.

\subsubsection{Groups Satisfying Finitely Many Identities}

In the style of [6, Section 2.2], we define an identity to be an element $w$ of a free group $E$ over some alphabet $\mathcal{Y}$. A group $G$ satisfies the identity $w \in E$, if for each homomorphism $\pi: E \rightarrow G$ we have that $w^{\pi}={ }_{G} 1$. For instance, all abelian groups satisfy the identity $w=[x, y]$. The free group over $\mathcal{X}$ in the variety of groups satisfying the identities $\left\{w_{1}, \ldots, w_{n}\right\} \subseteq E$ is

$$
\mathcal{F}=F /\left\langle w_{1}^{\pi}, \ldots, w_{n}^{\pi} \mid \pi \in \operatorname{Hom}(E, F)\right\rangle^{F}
$$

where $F$ denotes the free group over $\mathcal{X}$. The group $\mathcal{F}$ is universal in the sense that every group generated by $\mathcal{X}$ and satisfying the identities $w_{1}, \ldots, w_{n}$ is a homomorphic image of $\mathcal{F}$. A finitely generated group $G$ is finitely presented in the variety of groups satisfying the identities $w_{1}, \ldots, w_{n}$ if the kernel $\mathcal{F} \rightarrow G$ is finitely generated as normal subgroup. The following proposition is easily derived from [6, Proposition 2.13]:

Proposition 2.25 Each group that is finitely presented in the variety of groups satisfying finitely many identities is finitely L-presented.

The proof of [6, Proposition 2.13] uses a variation of Nielsen transformations. It generalizes to further examples of finitely $L$-presented groups; see Section 2.6 .3 below. For instance, the free Burnside group $B(m, n)$ on $m$ generators that satisfies the identity $x^{n}$ is finitely $L$-presented; see the AMS review of [114. Another example is given explicitly by the following $L$-presentation from [64]: 
Example 2.26 An $n$-Engel group is a group $G$ that satisfies the $n$-th Engel identity $\left[t,,_{n} u\right]={ }_{G} 1$, for all $t, u \in G$, where $\left[t,_{n} u\right]$ is defined recursively by $\left[t,,_{1} u\right]=[t, u]$ and $[t, n+1 u]=[[t, n u], u]$.

The free group $E(m, n)$ in the variety of m-generated $n$-Engel groups is finitely $L$ presented by $\left\langle\left\{x_{1}, \ldots, x_{m}\right\} \cup\{t, u\}|\{t, u\}| \Phi \mid\left\{\left[t,,_{n} u\right]\right\}\right\rangle$ where the substitutions $\Phi=$ $\left\{\sigma_{i}^{+}, \sigma_{i}^{-}, \tau_{i}^{+}, \tau_{i}^{-} \mid 1 \leq i \leq m\right\}$ are induced by the maps

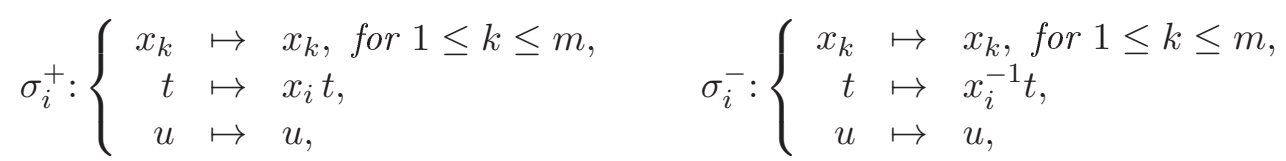

and

$$
\tau_{i}^{+}:\left\{\begin{aligned}
x_{k} & \mapsto x_{k}, \text { for } 1 \leq k \leq m, \quad \tau_{i}^{2}:\left\{\begin{aligned}
x_{k} & \mapsto x_{k}, \text { for } 1 \leq k \leq m, \\
t & \mapsto t, \\
u & \mapsto x_{i} u,
\end{aligned}\right. \text { 界, } \\
u & \mapsto x_{i}^{-1} u,
\end{aligned}\right.
$$

respectively.

\subsubsection{Further Examples of Finitely $L$-Presented Groups}

The notion of a finite $L$-presentation is quite natural. It was used intrinsically in many instances for the construction of groups with interesting properties. The constructive proof of Theorem 2.25 (as indicated in the Example 2.26), allows one to prove that many infinitely presented groups in literature are finitely $L$-presented. For instance, the lamplighter group $\mathbb{Z}_{2} \prec \mathbb{Z}$ admits a finite $L$-presentation:

Proposition 2.27 If $H$ is a finitely presented group, the wreath product $H \backslash \mathbb{Z}$ is finitely L-presented.

Proof. Let $\langle\mathcal{X} \mid \mathcal{R}\rangle$ be a finite presentation for the group $H$. Then the wreath product $H \imath \mathbb{Z}$ admits the presentation

$$
\left\langle\mathcal{X} \cup\{t\} \mid \mathcal{R} \cup\left\{\left[x^{t^{i}}, y^{t^{j}}\right] \mid x, y \in \mathcal{X}, i, j \in \mathbb{Z}, i \neq j\right\}\right\rangle .
$$

It is easy to see that we can replace the relations $\left[x^{t^{i}}, y^{t^{j}}\right]=\left[x, y^{t^{j-i}}\right]^{t^{i}}$, with $i, j \in \mathbb{Z}$, by the relations $\left[x, y^{t^{i}}\right]$ with $i \in \mathbb{N} \backslash\{0\}$ without changing the isomorphism type of the group. In particular, we have that

$$
H\left\langle\mathbb{Z} \cong\left\langle\mathcal{X} \cup\{t\} \mid \mathcal{R} \cup\left\{\left[x, y^{t^{i}}\right] \mid x, y \in \mathcal{X}, i \in \mathbb{N} \backslash\{0\}\right\}\right\rangle .\right.
$$

For each $x \in \mathcal{X}$, we introduce a stable letter $u_{x} \notin \mathcal{X}$ and we write $\mathcal{U}=\left\{u_{x} \mid x \in \mathcal{X}\right\}$ so that $\mathcal{X} \cap \mathcal{U}=\emptyset$ holds. Consider the finite $L$-presentation

$$
\left\langle\mathcal{X} \cup \mathcal{U} \cup\{t\}\left|\left\{x^{-1} u_{x}\right\}_{x \in \mathcal{X}}\right|\left\{\sigma_{x}\right\}_{x \in \mathcal{X}} \mid \mathcal{R} \cup\left\{\left[x, u_{y}^{t}\right]\right\}_{x, y \in \mathcal{X}}\right\rangle,
$$


where $\sigma_{y}$ denotes the endomorphism of the free group over $\mathcal{X} \cup \mathcal{U} \cup\{t\}$ that is induced by the map

$$
\sigma_{y}:\left\{\begin{aligned}
x & \mapsto x, \quad \text { for each } x \in \mathcal{X} \\
t & \mapsto t \\
u_{x} & \mapsto u_{x}, \quad \text { for each } x \in \mathcal{X} \backslash\{y\} \\
u_{y} & \mapsto\left(u_{y}\right)^{t} .
\end{aligned}\right.
$$

The map $x \mapsto x, t \mapsto t$, and $u_{x} \mapsto x$ induces a surjective homomorphism from the group defined by the finite $L$-presentation in Eq. (2.5) onto the wreath product in Eq. (2.4). The map that identifies the generators $\mathcal{X} \cup\{t\}$ of the wreath product in Eq. (2.4) with the corresponding generators of the finite $L$-presentation in Eq. (2.5) defines a surjective homomorphism. These homomorphisms are clearly inverses of each other. Thus the finite $L$-presentation in Eq. (2.5) defines $H$ < $\mathbb{Z}$.

The finite $L$-presentation for the wreath product in Eq. (2.5) is not ascending. In general, we are not aware of an ascending $L$-presentation for the wreath products $H \imath \mathbb{Z}$. However, in contrast to [6], Bartholdi noticed that the lamplighter group $\mathbb{Z}_{2} \prec \mathbb{Z}$ is finitely $L$ presented by

$$
\mathbb{Z}_{2} \prec \mathbb{Z} \cong\left\langle\{a, t\}|\emptyset|\{\sigma\} \mid\left\{a^{2},\left[a, a^{t}\right]\right\}\right\rangle,
$$

where $\sigma$ denotes the endomorphism of the free group over $\{a, t\}$ that is induced by the map $a \mapsto a^{t} a$ and $t \mapsto t$. We generalize this construction in the following proposition:

Proposition 2.28 If $H$ is a finitely generated abelian group, the wreath product $H \imath \mathbb{Z}$ is invariantly finitely L-presented.

Proof. For a proof, we refer to Proposition 2.3 in Appendix B.

Another group that is finitely $L$-presented was constructed in [85]: Consider the subgroup $H=\left\langle a_{1}, \ldots, a_{r}\right\rangle$ of the group $G=\left\langle\left\{a_{1}, \ldots, a_{r}, t\right\} \mid\left\{w_{1}, \ldots, w_{s}, a_{1}^{t} v_{1}^{-1}, \ldots, a_{r}^{t} v_{r}^{-1}\right\}\right\rangle$ where $w_{j}$ are freely and cyclically reduced words in $\mathcal{X}^{ \pm}=\left\{a_{1}, \ldots, a_{r}\right\}^{ \pm}$. If $G$ satisfies some small-cancellation condition, $H$ is not finitely presented 85. However, by Theorem 3.1 of [85], $H$ is finitely $L$-presented by $\left\langle\left\{a_{1}, \ldots, a_{r}\right\}|\emptyset|\{\phi\} \mid\left\{w_{1}, \ldots, w_{r}\right\}\right\rangle$ where $\phi$ denotes the endomorphism of the free group $F$ over $\left\{a_{1}, \ldots, a_{r}\right\}$ that is induced by the map

$$
\phi: F \rightarrow F,\left\{\begin{array}{ccc}
a_{1} & \mapsto & v_{1}, \\
& \vdots & \\
a_{r} & \mapsto & v_{r} .
\end{array}\right.
$$

Similarly, the finitely generated group with trivial Schur multiplier in [18 is finitely $L$-presented but not finitely presented. We are not aware of an invariant finite $L$ presentation for this group [64]. Another example of a finite $L$-presentation is given by the recursive presentation for Mihailova's subgroup in [26].

\subsection{Decision Problems for Finitely $L$-Presented Groups}

In this section, we consider decision problems for finitely $L$-presented groups. Since each finite presentation can be considered as a finite $L$-presentation, we have the following 
Remark 2.29 If an abstract property is not recursively recognizable for finitely presented groups, it is not recursively recognizable for finitely L-presented groups.

Since a finitely $L$-presented group is recursively presented, the following lemma applies to finitely $L$-presented groups:

Lemma 2.30 The word problem of a recursively presented group is semi-decidable.

Proof. Let $\langle\mathcal{X} \mid \mathcal{R}\rangle$ be a recursive presentation of a group. Suppose that $r_{1}, r_{2}, \ldots$ is a recursive enumeration of the group's relations; i.e., $r_{n} \in \mathcal{R}$ denotes the element that is returned as the $n$-th step of the algorithm that list the elements of $\mathcal{R}$ one after another. The word problem is semi-decidable if the set of elements $g \in\langle\mathcal{R}\rangle^{F}$ is recursively enumerable where $F$ denotes the free group over $\mathcal{X}$. For $g \in\langle\mathcal{R}\rangle^{F}$, we have $g=u_{1}^{-1} r_{1}^{\varepsilon_{1}} u_{1} \cdots u_{m}^{-1} r_{m}^{\varepsilon_{m}} u_{m}$ with $r_{i} \in \mathcal{R}, u_{i} \in F$, and $\varepsilon= \pm 1$. Since the free group $F$ is finitely generated by $\mathcal{X}$, elements of this form are recursively enumerable and thus $g \in\langle\mathcal{R}\rangle^{F}$ can be listed one after another.

However, there are recursively presented groups whose word problem is not decidable 95 . Section IV.7]. In the following, we consider two decision problems which could be faced with our algorithms in special cases. Decision problems for recursively presented groups were also studied in [94].

\subsubsection{Finite Presentability Problem}

Because 'being finitely presented' does not depend on the generating set of the group, it is an abstract property [38]. Since the class of finitely presented groups is contained in the class of finitely $L$-presented groups, a natural decision problem for a finitely $L$ presented group is to ask for an algorithm that decides whether or not a given finite $L$-presentation defines a finitely presented group. In general, this finite presentability problem for finitely $L$-presented groups is not decidable. In order to prove that the finite presentability problem is not decidable, we consider

Theorem 2.31 (Baumslag [17]) For finitely presented groups $G$ and $H$, the wreath product $H>G$ is finitely presented if and only if either $H$ is trivial or $G$ is finite.

An immediate consequence of Proposition 2.27 and Theorem 2.31 is the following

Proposition 2.32 There exists no algorithm that decides whether or not a finite $L$ presentation defines a finitely presented group.

Proof. Let $H=\langle\mathcal{X} \mid \mathcal{R}\rangle$ be a finitely presented group. By Proposition 2.27 the wreath product $H \backslash \mathbb{Z}$ is finitely $L$-presented. By Theorem 2.31 , though, $H \backslash \mathbb{Z}$ is finitely presented if and only if $H$ is trivial. If an algorithm existed for deciding whether or not a finite $L$-presentation defines a finitely presented group, there would exist an algorithm for deciding whether or not $H$ is trivial. However, 'being trivial' is a Markov property. By the Adian-Rabin Theorem 2.6, it is therefore impossible to decide whether or not a finite $L$-presentation defines a finitely presented group.

As every finitely $L$-presented group is recursively presented, Proposition 2.32 yields 
Corollary 2.33 The finite presentability problem for a recursively presented group is not decidable.

Even though it is not decidable whether or not a finite $L$-presentation defines a finitely presented group, the algorithm in [66] or Appendix E addresses this decision problem practically. It attempts to gain computational evidence for an invariantly finitely $L$ presented group to be infinitely related. Our approach follows the ideas from [56]. It computes successive (still finitely generated) factor groups of the Schur multiplier of an invariantly finitely $L$-presented group.

The Schur multiplier $M(G)$ of a group $G$ is an invariant which is related to a group presentation by Hopf's formula: If $F$ is a free group and $R \unlhd F$ is a normal subgroup so that $G=F / R$ holds, the Schur multiplier $M(G)$ satisfies

$$
M(G) \cong\left(R \cap F^{\prime}\right) /[R, F] .
$$

The Schur multiplier $M(G)$ is related to the finite presentability problem:

Lemma 2.34 The Schur multiplier of a finitely presented group is a finitely generated abelian group.

Proof. If $G$ is finitely presented, there exists a finite presentation $\langle\mathcal{X} \mid \mathcal{R}\rangle$ for $G$. Let $F$ be the free group over $\mathcal{X}$ and denote by $R$ the normal closure in $F$ of the finitely many relations in $\mathcal{R}$. Then $G=F / R$ holds. By Hopf's formula, the Schur multiplier satisfies $M(G) \cong\left(R \cap F^{\prime}\right) /[R, F]$. The subgroup $\left(R \cap F^{\prime}\right) /[R, F]$ is contained in the central subgroup $R /[R, F] \leq F /[R, F]$. Since $R$ is finitely generated as normal subgroup, $R /[R, F]$ is a finitely generated abelian group. Thus, all subgroups of $R /[R, F]$ are finitely generated. In particular, the Schur multiplier $M(G) \cong\left(R \cap F^{\prime}\right) /[R, F] \leq R /[R, F]$ is a finitely generated abelian group.

Lemma 2.34 was used in [56] to prove that the group $\mathfrak{G}$ constructed by Grigorchuk 53 ] is not finitely presented. These explicit calculations were generalized to an algorithm for computing Dwyer quotients of the Schur multiplier in [66] or Appendix E.

Notice that the converse of Lemma 2.34 is not true: A counter-example, namely a group with finitely generated (even trivial) Schur multiplier which is not finitely presented, was constructed in [18]:

Theorem 2.35 (Baumslag [18]) The group

$$
B=\left\langle\{a, b, t\} \mid\left\{a^{t} a^{-4}, b^{t^{-1}} b^{-2},\left[a, b^{t^{i}}\right] \mid i \in \mathbb{Z}\right\}\right\rangle
$$

is a met-abelian, infinitely related group with trivial Schur multiplier.

The constructions in the proof of Proposition 2.27 also show that Baumslag's group in Theorem 2.35 is finitely $L$-presented; see [64].

Even though the Schur multiplier of a finitely $L$-presented group is not computable in general [52], our approach in [66] or Appendix E computes successive quotients, so-called Dwyer quotients, of the Schur multiplier of an invariantly finitely $L$-presented group. 
Various self-similar groups admit striking patterns along the successive Dwyer quotients which suggest that their Schur multiplier is infinitely generated. The algorithm in [66] or Appendix E can be seen as a quotient method for the Schur multiplier of an invariantly finitely $L$-presented group.

\subsubsection{The Group Homomorphism Problem}

In this section, we consider a computational problem which is fundamental for any quotient method for finitely $L$-presented groups. Even though an answer to this problem may depend on the $L$-presentation, a solution to this problem allows us to develop interesting algorithms for finitely $L$-presented groups; e.g. the coset enumerator in [67] is a consequence of our solution for the class of finite groups.

Let $\mathcal{H}$ denote a class of groups (e.g. an important case is that $\mathcal{H}$ is the class of finite groups). Suppose that $G$ is finitely $L$-presented by $\langle\mathcal{X}|\mathcal{Q}| \Phi \mid \mathcal{R}\rangle$ and assume that $f: \mathcal{X} \rightarrow H$ is a map into a group $H \in \mathcal{H}$. We say that the group homomorphism problem for the class $\mathcal{H}$ is decidable if there exists an algorithm that decides whether or not the map $f$ extends to a homomorphism $G \rightarrow H$.

By the universal property of free groups, the map $f: \mathcal{X} \rightarrow H$ extends to a unique group homomorphism $\varphi: F \rightarrow H$ from the free group $F$ over the alphabet $\mathcal{X}$. The homomorphism $\varphi$ induces a group homomorphism $G \rightarrow H$ if and only if $G$ 's relations are mapped trivially; i.e., $\mathcal{Q} \cup \bigcup_{\sigma \in \Phi^{*}} \mathcal{R}^{\sigma} \subseteq \operatorname{ker}(\varphi)$ holds. A solution to the group homomorphism problem may depend on the class of groups.

If $H \in \mathcal{H}$ has a decidable word problem, there exists an algorithm that decides whether or not an element $r \in F$ satisfies $r^{\varphi}={ }_{H} 1$. Therefore, for a finitely presented group, the group homomorphism problem for a class $\mathcal{H}$ of groups with a (semi-)decidable word problem is (semi-)decidable because it suffices to check finitely many relations. If the group is finitely $L$-presented, though, there are possibly infinitely many relations that need to be considered for solving the group homomorphism problem. In Chapter 3, we obtain a solution to the group homomorphism problem for the class of finite groups:

Theorem 2.36 For a finitely L-presented group, the group homomorphism problem for the class of finite groups is decidable.

We also solve the group homomorphism problem for the class of finitely generated nilpotent groups (and even the class of polycyclic groups is likely to admit a solution to the group homomorphism problem). In general, though, we have no solution to the group homomorphism problem.

\subsection{First Results for Finitely $L$-Presented Groups}

In this section, we summarize some well-known results on the structure of a finitely $L$ presented group. The following group theoretic constructions preserve the property of being finitely $L$-presented: 
Theorem 2.37 (Bartholdi [6]) For finitely L-presented groups $G$ and $H$, the following groups are finitely L-presented:

- every factor group $G / N$ for a normal subgroup $N \unlhd G$ which is finitely generated as a normal subgroup of $G$,

- every split-extension $H \rtimes G$,

- every group extension of $G$ by a finitely presented group,

- the free product $G * H$, and

- every HNN-extension of $G$ relative to an isomorphism $\phi: K \rightarrow L$ where $K$ and $L$ are finitely generated subgroups of $G$.

An immediate consequence of Theorem 2.37 is

Corollary 2.38 Let $G$ and $H$ be finitely L-presented groups. If $F$ is a finitely generated group with embeddings $\psi: F \rightarrow G$ and $\phi: F \rightarrow H$, the amalgamated free product $G *_{F} H$ is finitely L-presented.

Furthermore, it was already suggested in [6] that each finite index subgroup of a finitely $L$-presented group is finitely $L$-presented. The proof in [6, Proposition 2.9] contains a gap which we addressed in [70] or Appendix D. However, finitely $L$-presented groups satisfy

Theorem 2.39 (Reidemeister-Schreier Theorem) Each finite index subgroup of a finitely L-presented group is finitely L-presented.

In [70] or Appendix D we also study conditions on a finite index subgroup of an invariantly finitely $L$-presented group to be invariantly finitely $L$-presented.

\subsection{Algorithms for Finitely $L$-Presented Groups}

It was shown in [9,64] that finite $L$-presentations allow computer algorithms to be applied in the investigation of finitely $L$-presented groups. So far, the only algorithm for finitely $L$-presented groups is the nilpotent quotient algorithm in [9, 64]:

\subsubsection{The Nilpotent Quotient Algorithm}

Most results on the structure of a self-similar group were obtained using their realization as automorphism groups of a regular rooted tree. For instance, the faithful action on the binary tree of group $\mathfrak{G}$ constructed by Grigorchuk [53] was used in [123] to compute its lower central series sections $\gamma_{c} \mathfrak{G} / \gamma_{c+1} \mathfrak{G}$; see also [10]. The lower central series of $\mathfrak{G}$ admits striking patterns so that the sections $\gamma_{c} \mathfrak{G} / \gamma_{c+1} \mathfrak{G}$ have a nice description (see Section A.3.1 below). In fact, $\mathfrak{G}$ is a group of finite width. Similar results were obtained in [7] for other self-similar groups including the Fabrykowski-Gupta group from [45, 46].

The striking patterns along the lower central series $\gamma_{c} G / \gamma_{c+1} G$ were a major motivation for generalizing the nilpotent quotient algorithm for finitely presented groups [107] 
to finitely $L$-presented groups in [9,64]. Similar to the original algorithm, this generalization computes a polycyclic presentation for the lower central series quotients $G / \gamma_{c}(G)$ of a finitely $L$-presented group $G$ together with a group homomorphism $G \rightarrow G / \gamma_{c} G$. The successful application of our nilpotent quotient algorithm to various self-similar groups yields detailed conjectures on the structure of their lower central series in general. Moreover, it provides a first algorithm for computing within the lower central series quotients $G / \gamma_{c} G$ of a finitely $L$-presented group (the groups $G / \gamma_{c} G$ have a decidable word problem). For further details on the nilpotent quotient algorithm for finitely $L$-presented groups, we refer to [9,64] or Section A.3 below.

Our generalization of the nilpotent quotient algorithm for finitely presented groups in [107] to finite $L$-presentations suggested that the $p$-quotient algorithm [74, 105] and the polycyclic quotient algorithm from [92,93] generalize to finite $L$-presentations [63]; see also [43. 


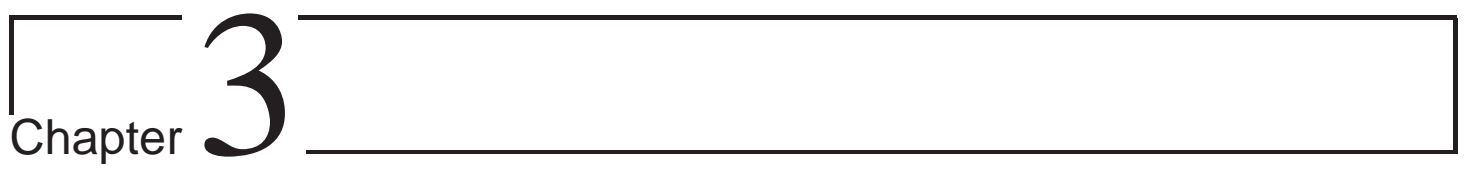

\section{The Group Homomorphism Problem}

A group presentation is a useful tool to decide if a map from a generating set of a group into another group induces a group homomorphism. More precisely, given a group presentation $\langle\mathcal{X} \mid \mathcal{R}\rangle$ of $G$ and a map $f: \mathcal{X} \rightarrow H$ from the generating set $\mathcal{X}$ of $G$ into a group $H$, the presentation is useful to decide whether or not $f$ induces a (unique) group homomorphism $G \rightarrow H$ : By the universal property of free groups, the map $f$ extends to a unique homomorphism $\varphi: F \rightarrow H$ from the free group $F$ over the alphabet $\mathcal{X}$ into the group $H$. The homomorphism $\varphi$ induces a group homomorphism $G \rightarrow H$ if and only if all relations in $\mathcal{R}$ of $G^{\prime}$ 's group presentation are mapped trivially. If $\langle\mathcal{X} \mid \mathcal{R}\rangle$ is a finite presentation, there are finitely many relations that need to be considered. The group homomorphism problem is decidable if both $G$ and $H$ are finite [90] and, even more general, if $G$ is finitely presented and $H$ has a decidable word problem. For finitely $L$-presented groups, though, there are possibly infinitely many relations that need to be checked in order to verify that $\varphi$ induces a group homomorphism $G \rightarrow H$.

In this chapter, we show that the group homomorphism problem is solvable if $H$ is finite or finitely generated and nilpotent. Our solution for the class of finite groups is the basis for our coset enumerator for finitely $L$-presented groups in 67] or Appendix C

\subsection{The Class of Finite Groups}

In this section, we consider the group homomorphism problem for the class $\mathcal{H}$ of finite groups. Let $G=\langle\mathcal{X}|\mathcal{Q}| \Phi \mid \mathcal{R}\rangle$ be a finitely $L$-presented group. We explicitly describe an algorithm that decides whether or not a map $f: \mathcal{X} \rightarrow H$ into a finite group $H \in \mathcal{H}$ induces a group homomorphism $G \rightarrow H$. Denote by $F$ the free group over the alphabet $\mathcal{X}$. By the universal property of free groups, $f$ extends (uniquely) to a homomorphism $\varphi: F \rightarrow H$. Then $\varphi$ induces a group homomorphism $\pi: G \rightarrow H$ if and only if all relations of $G$ 's finite $L$-presentation are mapped trivially by $\varphi$; i.e., we have $r^{\varphi}={ }_{H} 1$ for all $r \in \mathcal{Q} \cup \bigcup_{\sigma \in \Phi^{*}} \mathcal{R}^{\sigma}$.

Since $G$ is finitely $L$-presented there are only finitely many fixed relations in $\mathcal{Q}$ that can be checked one after another. Therefore, it remains to consider the (possibly infinitely 
many) relations $\bigcup_{\sigma \in \Phi^{*}} \mathcal{R}^{\sigma}$. We show that finitely many relations of the form $r^{\sigma}$, with $r \in \mathcal{R}$ and $\sigma \in \Phi^{*}$, are sufficient in order to decide whether or not $f: \mathcal{X} \rightarrow H$ induces to a group homomorphism $G \rightarrow H$. First, we consider the following

Lemma 3.1 For a finitely generated free group $F$ and a positive integer $n \in \mathbb{N}$, there are only finitely many subgroups in $F$ with index at most $n$.

Proof. Let $F$ be a finitely generated free group and let $n \in \mathbb{N}$ be given. Suppose that $U \leq F$ has finite index $m=[F: U] \leq n$. Then $F$ acts transitively on the right-cosets $U \backslash F$. Therefore, there exists a group homomorphism $\pi_{U}: F \rightarrow \operatorname{Sym}(U \backslash F) \cong \mathcal{S}_{m}$. On the other hand, each group homomorphism $\pi: F \rightarrow \mathcal{S}_{m}$ whose image $F^{\pi}$ acts transitively on the points $\{1, \ldots, m\}$ corresponds to a subgroup $U=\operatorname{Stab}_{F}(1)$ of $F$ with index $m$. In fact, there is a one-to-one correspondence between the subgroups with index $m$ and the group homomorphisms $\pi: F \rightarrow \mathcal{S}_{m}$ whose image acts transitively on $\{1, \ldots, m\}$.

By the universal property of a free group, a group homomorphism $\pi: F \rightarrow \mathcal{S}_{m}$ is uniquely defined by the images of the basis $\mathcal{X}$ of $F$. Since $F$ is finitely generated and $\mathcal{S}_{m}$ is finite, there are only finitely many homomorphisms in $\operatorname{Hom}\left(F, \mathcal{S}_{m}\right)$. Thus, there are only finitely many subgroups $U \leq F$ with index $m \leq n$.

Since every finitely generated group is a homomorphic image of a finitely generated free group, this yields the following

Corollary 3.2 For a finitely generated group $G$ and a positive integer $n \in \mathbb{N}$, there are only finitely many subgroup $H \leq G$ with $[G: H] \leq n$.

In the following, we consider endomorphisms $\sigma \in \operatorname{End}(F)$ of the free group $F$ over $\mathcal{X}$ and the kernels $\operatorname{ker}(\sigma \varphi)$ where $\varphi: F \rightarrow H$ denotes the (unique) extension of the map $f: \mathcal{X} \rightarrow H$ to the free group $F$.

Lemma 3.3 For endomorphisms $\sigma, \delta \in \operatorname{End}(F)$ of the free group $F$ and a homomorphism $\varphi: F \rightarrow H$ into a finite group, there exists an algorithm that decides whether or not $\operatorname{ker}(\sigma \varphi) \leq \operatorname{ker}(\delta \varphi)$ holds.

Proof. Since $H$ is finite and $\sigma$ is an endomorphism of the free group $F$, the image $\operatorname{im}(\sigma \varphi) \cong F / \operatorname{ker}(\sigma \varphi)$ is finite. Therefore, $\operatorname{ker}(\sigma \varphi)$ has finite index in $F$. The group $F$ acts transitively on $F / \operatorname{ker}(\sigma \varphi)$ and there exists an isomorphism $\iota: F / \operatorname{ker}(\sigma \varphi) \rightarrow \operatorname{im}(\sigma \varphi)$. This isomorphism allows us to compute a Schreier transversal $T$ for $\operatorname{ker}(\sigma \varphi)$ in $F$. Then Schreier's theorem (as, for instance, in [95, Proposition I.3.7]) allows us to compute a finite basis $\mathcal{Y}$ for the free subgroup $\operatorname{ker}(\sigma \varphi)$. The Schreier generators $\mathcal{Y}$ give a method to decide whether or not $\operatorname{ker}(\sigma \varphi) \leq \operatorname{ker}(\delta \varphi)$ holds: it suffices to evaluate the images $y^{\delta \varphi}$ for finitely many Schreier generators $y \in \mathcal{Y}$. Clearly, we have $\operatorname{ker}(\sigma \varphi) \leq \operatorname{ker}(\delta \varphi)$ if and only if $y^{\delta \varphi}={ }_{H} 1$ holds for each $y \in \mathcal{Y}$. Since $H$ is finite, it has a solvable word problem and thus $y^{\delta \varphi}={ }_{H} 1$ is decidable.

The condition $\operatorname{ker}(\sigma \varphi) \leq \operatorname{ker}(\delta \varphi)$ in Lemma 3.3 yields the following

Lemma 3.4 There exists a homomorphism $\pi: \operatorname{im}(\sigma \varphi) \rightarrow \operatorname{im}(\delta \varphi)$ with $\delta \varphi=\sigma \varphi \pi$ if and only if $\operatorname{ker}(\sigma \varphi) \leq \operatorname{ker}(\delta \varphi)$ holds. 
Proof. Suppose that there exists a homomorphism $\pi: \operatorname{im}(\sigma \varphi) \rightarrow \operatorname{im}(\delta \varphi)$ with $\delta \varphi=$ $\sigma \varphi \pi$. If $g \in \operatorname{ker}(\sigma \varphi)$, then $1=1^{\pi}=\left(g^{\sigma \varphi}\right)^{\pi}=g^{\sigma \varphi \pi}=g^{\delta \varphi}$. Thus $g \in \operatorname{ker}(\delta \varphi)$. If, on the other hand, $\operatorname{ker}(\sigma \varphi) \leq \operatorname{ker}(\delta \varphi)$ holds, there are isomorphisms $F / \operatorname{ker}(\sigma \varphi) \rightarrow$ $\operatorname{im}(\sigma \varphi), g \operatorname{ker}(\sigma \varphi) \mapsto g^{\sigma \varphi}$ and $F / \operatorname{ker}(\delta \varphi) \rightarrow \operatorname{im}(\delta \varphi), g \operatorname{ker}(\delta \varphi) \mapsto g^{\delta \varphi}$. Since $\operatorname{ker}(\sigma \varphi) \leq$ $\operatorname{ker}(\delta \varphi)$ holds, there is a natural homomorphism $F / \operatorname{ker}(\sigma \varphi) \rightarrow F / \operatorname{ker}(\delta \varphi), g \operatorname{ker}(\sigma \varphi) \mapsto$ $g \operatorname{ker}(\delta \varphi)$. A combination of these homomorphisms yields a homomorphism $\pi: \operatorname{im}(\sigma \varphi) \rightarrow$ $\operatorname{im}(\delta \varphi), g^{\sigma \varphi} \mapsto g^{\delta \varphi}$ that satisfies $\sigma \varphi \pi=\delta \varphi$.

An alternative proof of Lemma 3.3 is given by the algorithm in [90]: This algorithm allows one to decide whether or not a map from a generating set of a finite group into finite group induces a group homomorphism. By Lemma 3.4, we have $\operatorname{ker}(\sigma \varphi) \leq \operatorname{ker}(\delta \varphi)$ if and only if there exists a homomorphism $\pi: \operatorname{im}(\sigma \varphi) \rightarrow \operatorname{im}(\delta \varphi)$ with $\delta \varphi=\sigma \varphi \pi$. For the latter problem, the algorithm in [90] applies.

Our algorithm for deciding whether or not $f: \mathcal{X} \rightarrow H$ induces a homomorphism $G \rightarrow$ $H$ is shown in Algorithm 3.1. It takes as input a finite $L$-presentation $\langle\mathcal{X}|\mathcal{Q}| \Phi \mid \mathcal{R}\rangle$ of $G$ and the homomorphism $\varphi: F \rightarrow H$. It decides whether or not $\varphi$ induces a group homomorphism $G \rightarrow H$. The procedure IsGroupHomomorphism in Algorithm 3.1 is

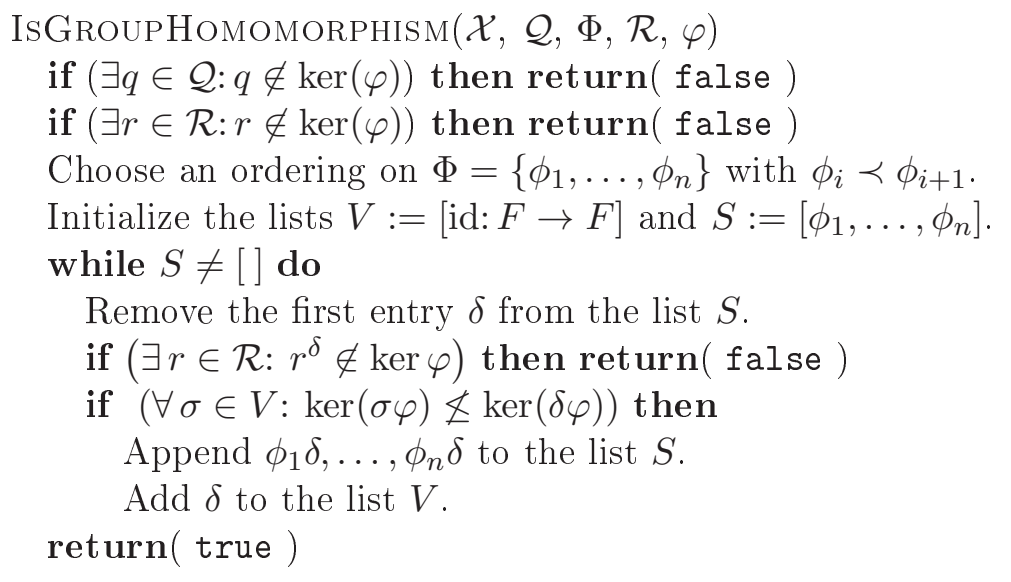

Algorithm 3.1: An algorithm to decide whether or not $\varphi: F \rightarrow H$ induces a group homomorphism $G \rightarrow H$.

an algorithm:

Lemma 3.5 The algorithm IsGroupHomomorphism in Algorithm 3.1 terminates and it either returns true or false.

Proof. Suppose that the procedure IsGroupHomomorphism does not terminate and hence, the while-loop will not terminate. Since the list $S=\left[\phi_{1}, \ldots, \phi_{n}\right]$ is finite and at each iteration of the while-loop an element is removed from $S$, non-termination of the procedure implies that it adds infinitely many elements $\delta$ to the list $V$. At each step, the elements $\delta, \sigma \in V$ satisfy $\operatorname{ker}(\sigma \varphi) \neq \operatorname{ker}(\delta \varphi)$. Because $\varphi: F \rightarrow H$ is a homomorphism into a finite group $H$ and $\operatorname{im}(\sigma \varphi) \leq \operatorname{im}(\varphi) \leq H$ holds, the images $\operatorname{im}(\sigma \varphi)$ and $\operatorname{im}(\delta \varphi)$ 
are finite groups. Since $F / \operatorname{ker}(\sigma \varphi) \cong \operatorname{im}(\sigma \varphi)$ holds, $\operatorname{ker}(\sigma \varphi)$ and $\operatorname{ker}(\delta \varphi)$ have finite index in $F$. As $F$ is finitely generated and $\operatorname{ker}(\sigma \varphi)$ has index at most $n=|\operatorname{im}(\varphi)|$, there are only finitely subgroups $\operatorname{ker}(\sigma \varphi)$ by Lemma 3.1. This contradicts our assumption that the procedure adds infinitely many elements $\sigma$ to $V$ with $\operatorname{ker}(\sigma \varphi) \neq \operatorname{ker}(\delta \varphi)$ for each pair $\delta, \sigma \in V$. Therefore, the while-loop eventually terminates and thus the procedure IsGroupHomomorphism is an algorithm. Clearly, it either returns true or false.

In order to prove that the algorithm IsGroupHomomorphism solves the group homomorphism problem for the class $\mathcal{H}$ of finite groups, we need the following observation:

Lemma 3.6 Suppose that the algorithm IsGroupHomomorphism returned true. For each $\delta \in \Phi^{*}$, there exists $\sigma \in V$ with $\operatorname{ker}(\sigma \varphi) \leq \operatorname{ker}(\delta \varphi)$.

Proof. The free monoid $\Phi^{*}$ is well-ordered with respect to the length-plus-lexicographic ordering which extends the ordering $\prec$ on the generating set $\Phi$. More precisely, the ordering $\prec$ of the finite generating set $\Phi=\left\{\phi_{1}, \ldots, \phi_{n}\right\}$ extends to the free monoid $\Phi^{*}$ as follows: define $\sigma \prec \delta$ if and only if either $\|\sigma\|<\|\delta\|$ holds or, otherwise, if $\sigma=\sigma_{1} \cdots \sigma_{n}$ and $\delta=\delta_{1} \cdots \delta_{n}$, with $\delta_{i}, \sigma_{i} \in \Phi$, and there exists $1 \leq k \leq n$ so that $\sigma_{i}=\delta_{i}$ for $k<i \leq n$ and $\sigma_{k} \prec \delta_{k}$. The obtained ordering $\prec$ on $\Phi^{*}$ is total and a well-ordering [129]. By construction, $S$ and $V$ in Algorithm 3.1 are always ordered with respect to this ordering. Moreover, at each step of the algorithm, the elements in $V$ precede those in $S$.

Suppose that $\delta \in \Phi^{*}$ is a $\prec$-minimal counter-example to this lemma. Then we have $\operatorname{ker}(\sigma \varphi) \not \leq \operatorname{ker}(\delta \varphi)$ for each $\sigma \in V$. At each step of the algorithm we neither have $\delta \in V$ nor $\delta \in S$ (if $\delta \in S$ at an intermediate step, the algorithm would either have found $\sigma \in V$ with $\operatorname{ker}(\sigma \varphi) \leq \operatorname{ker}(\delta \varphi)$ or it would have added $\delta$ to $V$ - in both cases we obtain a contradiction to our assumption because no elements were removed from $V$ ). Since the stack $S$ was initialized as $S:=\Phi$, we have that $\|\delta\| \geq 2$. Therefore, we can write $\delta=\delta_{1} \delta_{2} \cdots \delta_{n}$ with each $\delta_{i} \in \Phi$. The algorithm yields the existence of a minimal positive integer $1<k \leq n$ so that, at some step of the algorithm, we have $\delta_{k} \delta_{k+1} \cdots \delta_{n} \in S$ but never $\delta_{k-1} \delta_{k} \cdots \delta_{n} \in S$. Hence, there exists $\tau \in V$ so that $\operatorname{ker}(\tau \varphi) \leq \operatorname{ker}\left(\delta_{k} \cdots \delta_{n} \varphi\right)$ holds. Because the elements in $V$ precede those in $S$, the element $\tau \in V$ satisfies that $\tau \prec \delta_{k} \cdots \delta_{n}$. By Lemma 3.4, $\operatorname{ker}(\tau \varphi) \leq \operatorname{ker}\left(\delta_{k} \cdots \delta_{n} \varphi\right)$ implies the existence of a homomorphism $\pi: \operatorname{im}(\tau \varphi) \rightarrow \operatorname{im}\left(\delta_{k} \cdots \delta_{n} \varphi\right)$ with $\tau \varphi \pi=\delta_{k} \cdots \delta_{n} \varphi$. We obtain that

$$
\delta \varphi=\delta_{1} \cdots \delta_{n} \varphi=\delta_{1} \cdots \delta_{k-1} \delta_{k} \cdots \delta_{n} \varphi=\delta_{1} \cdots \delta_{k-1} \tau \varphi \pi .
$$

By Lemma 3.4, this yields that $\operatorname{ker}\left(\delta_{1} \cdots \delta_{k-1} \tau \varphi\right) \leq \operatorname{ker}(\delta \varphi)$. As $\tau \prec \delta_{k} \cdots \delta_{n}$ holds, we have that $\delta_{1} \cdots \delta_{k-1} \tau \prec \delta_{1} \cdots \delta_{k-1} \delta_{k} \cdots \delta_{n}=\delta$. If there existed $\sigma^{\prime} \in V$ with $\operatorname{ker}\left(\sigma^{\prime} \varphi\right) \leq$ $\operatorname{ker}\left(\delta_{1} \cdots \delta_{k-1} \tau \varphi\right)$, we would have that $\operatorname{ker}\left(\sigma^{\prime} \varphi\right) \leq \operatorname{ker}\left(\delta_{1} \cdots \delta_{k-1} \tau \varphi\right) \leq \operatorname{ker}(\delta \varphi)$. Hence, $\delta_{1} \cdots \delta_{k-1} \tau$ is a also counter-example to our lemma which precedes the counter-example $\delta$. This however contradicts the minimality of $\delta$.

We are now in a position to prove the following theorem:

Theorem 3.7 For a finitely L-presented group and the class of finite group $\mathcal{H}$, the group homomorphism problem for $\mathcal{H}$ is solved by the algorithm IsGROupHomomorPHism. 
Proof. Let $\varphi: F \rightarrow H$ be the unique homomorphism from the free group over the alphabet $\mathcal{X}$ into the finite group $H \in \mathcal{H}$ that lifts the map $f: \mathcal{X} \rightarrow H$. By Lemma 3.5, the algorithm IsGroupHomomorphism terminates and it either returns true or false.

If the algorithm returned $f$ alse, it found either a fixed relation $q \in \mathcal{Q}$ with $q^{\varphi} \neq 1$ or it found an iterated relation $r \in \mathcal{R}$ and a substitution $\sigma \in \Phi^{*}$ with $\left(r^{\sigma}\right)^{\varphi} \neq 1$. In this case, the homomorphism $\varphi: F \rightarrow H$ does not factor through to a group homomorphism $G \rightarrow H$.

Suppose that the algorithm IsGroupHomomorphism returned true. As the finitely many fixed relations in $\mathcal{Q}$ were verified by the algorithm, it suffices to check the (possibly infinitely many) iterated relations $r^{\delta}$ with $r \in \mathcal{R}$ and $\delta \in \Phi^{*}$ : By Lemma 3.6, there exists $\sigma \in V$ so that $\operatorname{ker}(\sigma \varphi) \leq \operatorname{ker}(\delta \varphi)$ holds. Thus, by Lemma 3.4, there exists a homomorphism $\pi: \operatorname{im}(\sigma \varphi) \rightarrow \operatorname{im}(\delta \varphi)$ with $\sigma \varphi \pi=\delta \varphi$. Since the algorithm did not return false and $\sigma \in V$ holds, the algorithm has verified that $r^{\sigma} \in \operatorname{ker}(\varphi)$ holds. We obtain $\left(r^{\delta}\right)^{\varphi}=r^{\delta \varphi}=r^{\sigma \varphi \pi}=\left(r^{\sigma \varphi}\right)^{\pi}=1^{\pi}=1$. Hence, all relations of the form $r^{\delta}$ with $r \in \mathcal{R}$ and $\delta \in \Phi^{*}$ are mapped trivially by $\varphi$. Therefore, $\varphi$ induces to a group homomorphism $G \rightarrow H$.

The algorithm IsGroupHomomorphism has various interesting applications. For instance, it allows us to develop a coset enumerator for finitely $L$-presented groups in [67] or Appendix C, This is an algorithm which, given a finitely generated subgroup of a finitely $L$-presented group, computes the index of the subgroup in the finitely $L$-presented group provided that this index is finite. Analogously to coset enumeration for finitely presented groups, our method has various useful further applications including a solution to the generalized word problem for finite index subgroups and a method for computing low-index subgroups of a finitely $L$-presented group.

\subsubsection{Applications of the algorithm IsGroupHomomorPHISM}

In this section, we summarize the applications of the algorithm IsGroupHomomorPHISM in Algorithm 3.1. In particular, it allows us to develop algorithms for subgroups of finitely $L$-presented groups:

- to compute the index of a finitely generated subgroup in a finitely $L$-presented groups provided that this index is finite,

- to compute a permutation representation for the group's action on the cosets of a finite index subgroup,

- to solve the generalized word problem for finite index subgroups,

- to compute the lattice of finite index subgroups of a finitely $L$-presented group,

- to compute a finite generating set of the kernel of a homomorphism from a finitely $L$-presented group into a finite group,

- to compute the intersection of two finite index subgroups, and

- to compute the normal core of a finite index subgroup. 
These methods have been implemented in the computer algebra system GAP [50]. Applications of these implementations to a class of self-similar groups are shown in Appendix A.

A fundamental application of algorithm IsGroupHomomorphism is the coset enumerator for finitely $L$-presented groups in [67] or Appendix C.

Proposition 3.8 If $G=\langle\mathcal{X}|\mathcal{Q}| \Phi \mid \mathcal{R}\rangle$ is a finitely L-presented group and $\mathcal{U}=\langle\mathcal{Y}\rangle$ is a finitely generated subgroup of $G$ which has finite index in $G$, there exists an algorithm that computes the index $[G: \mathcal{U}]$ together with a permutation representation $\varphi: G \rightarrow \operatorname{Sym}(\mathcal{U} \backslash G)$ for the group's action on the cosets.

Proof. Let $G=\langle\mathcal{X}|\mathcal{Q}| \Phi \mid \mathcal{R}\rangle$ be a finitely $L$-presented group and let $\mathcal{U}=\langle\mathcal{Y}\rangle$ be a finitely generated subgroup with finite index in $G$. Denote by $F$ the free group over $\mathcal{X}$ and write $K=\left\langle\mathcal{Q} \cup \bigcup_{\sigma \in \Phi^{*}} \mathcal{R}^{\sigma}\right\rangle^{F}$. The usual word-length of an element $\sigma \in \Phi^{*}$ from the free monoid $\Phi^{*}$ is denote by $\|\sigma\|$. The finitely presented groups $H_{i}=\left\langle\mathcal{X} \mid \mathcal{Q} \cup \bigcup_{\sigma \in \Phi_{i}} \mathcal{R}^{\sigma}\right\rangle$ with $\Phi_{i}=\left\{\sigma \in \Phi^{*} \mid\|\sigma\| \leq i\right\}$ naturally map onto $G$. We consider the finitely many generators $\mathcal{Y}$ of the subgroup $\mathcal{U}$ as elements of the free group $F$. Then $E=\langle\mathcal{Y}\rangle$ is a subgroup of $F$ with $\mathcal{U} \cong E K / K$. In particular, $E K$ has finite index in $F$. Since $F$ is finitely generated and $E K$ has finite index, $E K$ is finitely generated by $g_{1}, \ldots, g_{n}$, say.

Write $K_{i}=\left\langle\mathcal{Q} \cup \bigcup_{\sigma \in \Phi_{i}} \mathcal{R}^{\sigma}\right\rangle^{F}$. Then $E K_{0} \leq E K_{1} \leq E K_{2} \leq \ldots \leq E K \leq F$ and $E K=\bigcup_{i>0} E K_{i}$. Since $E K=\left\langle g_{1}, \ldots, g_{n}\right\rangle$ is finitely generated and $E K=\bigcup_{i>0} E K_{i}$ holds, there exists $\ell \in \mathbb{N}_{0}$ so that $E K_{\ell}=E K$. In fact, the subgroup $\mathcal{U}$ can be considered as a finite index subgroup of the finitely presented group $H_{\ell}=\left\langle\mathcal{X} \mid \mathcal{Q} \cup \bigcup_{\sigma \in \Phi_{\ell}} \mathcal{R}^{\sigma}\right\rangle$. We are to compute the index $[G: \mathcal{U}]=\left[F: E K_{\ell}\right]=\left[H_{\ell}: \mathcal{U}\right]$ together with the permutation representation $\varphi: F \rightarrow \operatorname{Sym}\left(E K_{\ell} \backslash F\right)$ which induces $G \rightarrow \operatorname{Sym}(\mathcal{U} \backslash G)$.

Coset enumeration for finitely presented groups computes a permutation representation $\varphi_{j}: F \rightarrow \operatorname{Sym}\left(E K_{j} \backslash F\right)$ provided that $\left[F: E K_{j}\right]$ is finite 1 In this case, the index $\left[F: E K_{j}\right]$ divides $\left[F: E K_{\ell}\right]$. It remains to prove that $\varphi_{j}: F \rightarrow \operatorname{Sym}\left(E K_{j} \backslash F\right)$ induces a homomorphism $G \rightarrow \operatorname{Sym}\left(E K_{j} \backslash F\right)$. Here, our algorithm IsGroupHomomorphism applies. In particular, it allows us to decide whether or not $\varphi_{j}$ induces a group homomorphism $G \rightarrow \operatorname{Sym}\left(E K_{j} \backslash F\right)$. If this is the case, the index $[G: \mathcal{U}]=\left[F: E K_{\ell}\right]$ and $\left[F: E K_{j}\right]$ coincide. Otherwise, we have to increase the index $j$. Termination of this process is guaranteed by the existence of $\ell \in \mathbb{N}$ so that $E K_{\ell}=E K$. For further details, we refer to [67] or Appendix [C.

An 'inverse' of the coset enumerator in Proposition 3.8 is given by Schreier's theorem (as, for instance, in [95, Proposition I.3.7]). It allows us to compute finitely many Schreier generators for a finite index subgroup of a finitely generated free group:

\footnotetext{
${ }^{1}$ Although the index $j$, so that $\left[F: E K_{j}\right]$ is finite, could not be given a priori, the following approach yields an algorithm whose termination could be guaranteed: For an arbitrary index $j$, the coset enumerator for finitely presented groups could be run with an upper bound $N$ on the number of intermediate cosets. If this process does not terminate successfully, we both increase the index $j$ and the upper bound $N$ on the intermediate cosets. We then run both algorithms in parallel. We continue increasing these numbers and to run these algorithms in parallel until eventually one of them terminates. Since the subgroup has finite index, termination of this process is guaranteed.
} 
Proposition 3.9 There exists an algorithm that computes a finite generating set for a finite index subgroup $\mathcal{U}$ of a finitely $L$-presented group $G$ from a permutation representation $\varphi: G \rightarrow \operatorname{Sym}(\mathcal{U} \backslash G)$.

Proof. The subgroup $\mathcal{U}$ coincides with the stabilizer of the trivial coset $\mathcal{U} 1$ under the action of $G$. The permutation representation $\varphi$ allows us to compute a finite generating set for the stabilizer $\operatorname{Stab}_{G}(\mathcal{U} 1)$ using an orbit-stabilizer algorithm.

Another application of coset enumeration is given by

Proposition 3.10 If $G=\langle\mathcal{X}|\mathcal{Q}| \Phi \mid \mathcal{R}\rangle$ is a finitely L-presented group, there exists an algorithm that proves that $G$ is finite and that computes the order $|G|$ provided that $G$ is finite.

Proof. If $G$ is finite, the trivial subgroup $\mathcal{U}=\langle\emptyset\rangle$ has finite index in $G$. Therefore, our coset enumerator terminates and it computes the index $[G: \mathcal{U}]=[G:\{1\}]=|G|$.

Similarly, coset enumeration yields the following

Proposition 3.11 If $G=\langle\mathcal{X}|\mathcal{Q}| \Phi \mid \mathcal{R}\rangle$ is a finitely L-presented group and $\mathcal{U}=\langle\mathcal{Y}\rangle$ is a finitely generated subgroup with finite index in $G$, the generalized word problem for $\mathcal{U}$ is decidable.

Proof. If $\mathcal{U}$ has finite index in $G$, coset enumeration computes a permutation representation $\varphi: F \rightarrow \operatorname{Sym}(E K \backslash F)$. Let $g \in G$ be given. We consider the element $g$ as an element of the free group $F$ over $\mathcal{X}$. Then $g \in E K$ if and only if $g \in \operatorname{Stab}_{\operatorname{Sym}(E K \backslash F)}(E K 1)$. Using the permutation representation $\varphi$, it is clearly decidable whether or not $g \in$ $\operatorname{Stab}_{\operatorname{Sym}(E K \backslash F)}(E K 1)$ holds.

This solution to the generalized word problem for finite index subgroups yields the following

Corollary 3.12 If $G=\langle\mathcal{X}|\mathcal{Q}| \Phi \mid \mathcal{R}\rangle$ is a finitely L-presented group and $\mathcal{U}=\langle\mathcal{Y}\rangle$ and $\mathcal{V}=\langle\mathcal{Z}\rangle$ are a finitely generated subgroups with finite index in $G$, there exists an algorithm that decides whether or not $\mathcal{U} \leq \mathcal{V}$ holds. If $\mathcal{U} \leq \mathcal{V}$ holds, there exists an algorithm that computes $[\mathcal{V}: \mathcal{U}]$. There exists an algorithm that decides whether or not $U \leq G$ is normal in $G$ and in this case computes a permutation representation for the finite group $G / U$.

The low-index subgroup algorithms for finitely presented groups in [129, Section 5.6] and [35,41] yield the following

Proposition 3.13 If $G=\langle\mathcal{X}|\mathcal{Q}| \Phi \mid \mathcal{R}\rangle$ is a finitely L-presented group, there exists an algorithm that computes the lattice of finite index subgroups of $G$.

Proof. For a positive integer $n>0$, the low-index subgroup algorithms in [35, 41] and [129, Section 5.6] enumerate the subgroups of a finitely presented group with index at most $n$ (in practice, the index $n$ is rather limited so that these algorithms are 
known as low-index subgroup algorithms). These algorithms enumerate those homomorphisms $F \rightarrow \mathcal{S}_{n}$ from the free group $F$ over $\mathcal{X}$ into the symmetric group $\mathcal{S}_{n}$ that factor through to a homomorphism $G \rightarrow \mathcal{S}_{n}$. They could be used to compute all subgroups with finite index in a finitely $L$-presented group $G=\langle\mathcal{X}|\mathcal{Q}| \Phi \mid \mathcal{R}\rangle$ as follows: Consider the notion introduced in the proof of Proposition 3.8, For an arbitrary $i \in \mathbb{N}_{0}$, consider the finitely presented group $H_{i}=\left\langle\mathcal{X} \mid \mathcal{Q} \cup \bigcup_{\sigma \in \Phi_{i}} \mathcal{R}^{\sigma}\right\rangle$. Each subgroup $E K_{i} \leq F$ of the finitely presented group $H_{i}$ can be computed with the low-index subgroup algorithms for finitely presented groups. The subgroup $E K_{i} / K_{i}$ of the finitely presented group $H_{i}$ naturally maps onto the subgroup $E K / K$ of the finitely $L$-presented group $G$. Therefore, every finite index subgroup $E K_{i} / K_{i}$ of the finitely presented group $H_{i}$ yields a finite index subgroup $E K / K$ of the finitely $L$-presented group. Clearly, the index $[F: E K]$ divides the index $\left[F: E K_{i}\right]$. On the other hand, every finite index subgroup $E K / K$ of the finitely $L$-presented group $G$ yields a finite index subgroup $E K / K_{i}$ of the finitely presented group $H_{i}$. In particular, every finite index subgroup of $G$ shows up in the list of finite index subgroups of $H_{i}$.

The list of finite index subgroups of the finitely presented group $H_{i}$ possibly contains duplicate subgroups of $G$. It therefore remains to remove duplicates from a list of subgroups. But this is an application of Corollary 3.12. Therefore, the list of subgroups of a finitely $L$-presented group with index at most $n$ can be computed from a list of subgroups with index at most $n$ of the finitely presented group $H_{i}$.

A low-index subgroup algorithm may help to prove that a finitely $L$-presented group is non-trivial if we can find a subgroup with non-trivial index in $G$; see [129, p.251]. Similar to finitely presented groups, we also have the following application:

Proposition 3.14 There exists an algorithm that computes a permutation representation for the group's action on the cosets of the intersection of two finite index subgroups of a finitely L-presented group.

Proof. Let $\varphi: F \rightarrow \operatorname{Sym}(\{1, \ldots, n\})$ and $\psi: F \rightarrow \operatorname{Sym}(\{1, \ldots, m\})$ be permutation representations for the group's action on the right-cosets $E K \backslash F$ and $V K \backslash F$ of two finite index subgroup of a finitely $L$-presented group. Then $F$ acts on the Cartesian product $\{1, \ldots, n\} \times\{1, \ldots, m\}$ diagonally via $(a, b)^{g}=\left(a^{g^{\varphi}}, b^{g^{\psi}}\right)$. The stabilizer of the point $(1,1)$ coincides with the intersection $E K \cap V K$. Since $\{1, \ldots, n\} \times\{1, \ldots, m\}$ is finite, an orbit-stabilizer algorithm computes a generating set for the stabilizer of the point $(1,1)$ and thus a generating set for the intersection $E K \cap V K$. A permutation representation for $E K \cap V K$ is then obtained from coset enumeration.

An immediate consequence of Proposition 3.14 is the following

Corollary 3.15 There exists an algorithm that computes a permutation representation for the group's action on the cosets of the normal core of a finite index subgroup of a finitely L-presented group.

Proof. The normal core $N_{\mathcal{U}}$ of a $\operatorname{subgroup} \mathcal{U} \leq G$ is the largest normal subgroup that is contained in $\mathcal{U}$ and it satisfies $N_{\mathcal{U}}=\bigcap_{g \in G} \mathcal{U}^{g}$. Since $[G: \mathcal{U}]$ is finite, there exists a finite 
transversal $T \subseteq G$ for $\mathcal{U}$ in $G$. Therefore, the normal core $N_{\mathcal{U}}$ satisfies $N_{\mathcal{U}}=\bigcap_{t \in T} \mathcal{U}^{t}$ and thus it is the intersection of finitely many finite index subgroups. By Proposition 3.14, a permutation representation for this intersection can be computed.

For a finitely $L$-presented group $G=\langle\mathcal{X}|\mathcal{Q}| \Phi \mid \mathcal{R}\rangle$ and a homomorphism $\varphi: G \rightarrow H$ into a finite group $H$, a finite generating set for the $\operatorname{kernel} \operatorname{ker}(\varphi)$ is computable:

Proposition 3.16 If $G=\langle\mathcal{X}|\mathcal{Q}| \Phi \mid \mathcal{R}\rangle$ is a finitely L-presented group and if $\varphi: G \rightarrow$ $H$ is a homomorphism into a finite group, there exists an algorithm that computes a finite generating set for the kernel of $\varphi$.

Proof. Since $H$ is finite, we can consider $H$ as a finite permutation group $H \leq \mathcal{S}_{n}=$ $\operatorname{Sym}(\{1, \ldots, n\})$. Moreover, we have $F / \operatorname{ker}(\varphi) \cong \operatorname{im}(\varphi) \leq \mathcal{S}_{n}$. By [95, Proposition 4.1], it suffices to compute a Schreier transversal for $\operatorname{ker}(\varphi)$ in $F$. Because there is a one-to-one correspondence between the cosets $F / \operatorname{ker}(\varphi)$ and the elements of $\operatorname{im}(\varphi)$, this Schreier transversal can be computed in the finite permutation group $\mathcal{S}_{n}$.

An application of the latter proposition allows us to compute a generating set for the derived subgroup $G^{\prime}=[G, G]$ provided that the abelianization $G / G^{\prime}$ is finite. Note that the abelianization is computable with the nilpotent quotient algorithm in [9, 64]. Combining this method with the Reidemeister-Schreier theorem for finitely $L$-presented groups in 70] or Appendix D, we obtain a solvable quotient algorithm for finitely $L$ presented groups:

Corollary 3.17 If $G=\langle\mathcal{X}|\mathcal{Q}| \Phi \mid \mathcal{R}\rangle$ is a finitely L-presented group, there exists an algorithm that computes the isomorphism type of the abelianization $G / G^{\prime}$. If $G / G^{(i)}$ is finite for some $i \in \mathbb{N}$, there exists an algorithm that computes the isomorphism type of the sections $G^{(i)} / G^{(i+1)}$.

Proof. The nilpotent quotient algorithm in [9, 64] computes a polycyclic presentation for $G / G^{\prime}$ together with a homomorphism $G \rightarrow G / G^{\prime}$. The polycyclic presentation for $G / G^{\prime}$ allows us to compute the isomorphism type of $G / G^{\prime}$. In particular, it allows us to decide whether or not $G / G^{\prime}$ is finite. Suppose that, for $i \geq 2$, the quotient $G / G^{(i)}$ is finite. Then Proposition 3.16 allows us to compute a finite generating set for the kernel $G^{(i)}=\operatorname{ker}\left(G \rightarrow G / G^{(i)}\right)$. Then the constructive proof of the Reidemeister-Schreier Theorem 2.39] in [70] or Appendix D allows us to compute a finite $L$-presentation for the finite index subgroup $G^{(i)} \leq G$. This finite $L$-presentation enables us to use the nilpotent quotient algorithm for computing the abelianization $G^{(i)} /\left[G^{(i)}, G^{(i)}\right]=G^{(i)} / G^{(i+1)}$ together with a homomorphism $G^{(i)} \rightarrow G^{(i)} / G^{(i+1)}$. If $G^{(i)} / G^{(i+1)}$ is finite, then so is $G / G^{(i+1)}$. The process continues until $G^{(i)} / G^{(i+1)}$ is either trivial or infinite.

The algorithms above have been implemented in the computer algebra system GAP [50]. Their implementations have been applied successfully to a class of self-similar group in Appendix $\mathrm{A}$ 


\subsection{The Class of Finitely Generated Nilpotent Groups}

In this section, we consider the group homomorphism problem for finitely generated nilpotent groups. This problem can be faced with the quotient method in [9,64: More precisely, for a finitely $L$-presented group $G$ and a positive integer $c$, the nilpotent quotient algorithm in 9, 64 computes a weighted nilpotent presentation for the lower central series quotient $G / \gamma_{c} G$ together with a homomorphism $\pi: F \rightarrow G / \gamma_{c} G$ which induces a homomorphism $G \rightarrow G / \gamma_{c} G$. A weighted nilpotent presentation is a finite presentation that admits an effective solution to the word problem in $G / \gamma_{c} G$ as it provides a confluent rewriting system.

The nilpotent quotient algorithm allows us to solve the group homomorphism problem for the class $\mathcal{H}$ of finitely generated nilpotent groups: Let $H \in \mathcal{H}$ be given. We assume that $H$ is given by a weighted nilpotent presentation (if $H$ is given by a finite presentation, a weighted nilpotent presentation can be computed with the algorithms in [9, 64, 107]). Suppose that $G=\langle\mathcal{X}|\mathcal{Q}| \Phi \mid \mathcal{R}\rangle$ is a finitely $L$-presented group and that $f: \mathcal{X} \rightarrow H$ is a map. We first compute the nilpotency class $c$ of the subgroup $L=\langle\{f(x) \mid x \in \mathcal{X}\}\rangle \leq H$. This can be done by first computing an induced polycyclic presentation for the subgroup. Then the nilpotent quotient algorithm for finitely presented groups applies. If $G$ has a maximal nilpotent quotient with nilpotency class less than $c, f$ cannot extend to a group homomorphism $G \rightarrow H$. We can therefore assume that the nilpotent quotient algorithm in [9, 64] computes a weighted nilpotent presentation for $G / \gamma_{c+1} G$ together with a homomorphism $\pi: F \rightarrow G / \gamma_{c+1} G$ which induces a homomorphism $G \rightarrow G / \gamma_{c+1} G$. There exists a homomorphism $G \rightarrow H$ if and only if there exists a homomorphism $\iota: G / \gamma_{c+1} G \rightarrow H$ so that the diagram

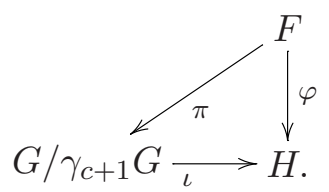

commutes. Since $G / \gamma_{c+1} G$ is finitely presented and $H$ has a decidable word problem, it is decidable whether or not such a homomorphism $\iota: G / \gamma_{c+1} G \rightarrow H$ exists.

The same ideas as above show that, since the polycyclic quotient algorithm for finitely presented groups in [92, 93] also generalizes to finitely $L$-presented groups [63], the group homomorphism problem for the class of polycyclic groups is decidable.

\subsection{Finitely Presented Images}

In this section, we consider the group homomorphism problem for the class of finitely presented groups with a solvable word problem. In a special case, we are also able to give a solution to this problem:

Proposition 3.18 Let $G=\langle\mathcal{X}|\mathcal{Q}| \Phi \mid \mathcal{R}\rangle$ be a finitely L-presented group and let $H=\langle\mathcal{Y} \mid \mathcal{S}\rangle$ be a finitely presented group whose word problem is decidable. Suppose that 
$f: \mathcal{X} \rightarrow H$ is a map so that $\langle f(\mathcal{X})\rangle$ has finite index in $H$. Let $\varphi: F \rightarrow H$ be the unique extension of $f$ to the free group $F$ over the alphabet $\mathcal{X}$. Then there exists an algorithm which decides whether or not $\operatorname{ker}(\varphi)$ is $\Phi$-invariant. In this case, there exists an algorithm which solves the group homomorphism problem for $H$.

Proof. By the Reidemeister-Schreier Theorem 2.2, every finite index subgroup of a finitely presented group is itself finitely presented. Moreover, as every subgroup of a finitely presented group with a solvable word problem has a solvable word problem, we can assume that $\varphi: F \rightarrow H$ is onto. Thus, for each $y \in \mathcal{Y}$, there exists a word $w_{y}\left(x_{1}, \ldots, x_{n}\right)$ in the generators $\mathcal{X}=\left\{x_{1}, \ldots, x_{n}\right\}$ so that $y={ }_{H} w_{y}\left(x_{1}, \ldots, x_{n}\right)^{\varphi}$ holds. Since $H$ has a decidable word problem, an enumeration of the elements of $F$ allow us to compute the representatives $w_{y}$. Moreover, every image $x^{\varphi}, x \in \mathcal{X}$, has the form $v_{x}\left(y_{1}, \ldots, y_{m}\right)$ where $\mathcal{Y}=\left\{y_{1}, \ldots, y_{m}\right\}$. Suppose that $\mathcal{X} \cap \mathcal{Y}=\emptyset$ holds. In the following, we use Tietze transformations to compute a finite presentation for the finitely presented group $H$ with generators $\mathcal{X}$. In particular, Tietze transformations yield that

$$
H \cong\left\langle\mathcal{X} \cup \mathcal{Y} \mid\left\{x^{-1} v_{x}\left(y_{1}, \ldots, y_{n}\right)\right\}_{x \in \mathcal{X}} \cup\left\{y^{-1} w_{y}\left(x_{1}, \ldots, x_{n}\right)\right\}_{y \in \mathcal{Y}} \cup \mathcal{S}\right\rangle .
$$

The relations $y^{-1} w_{y}\left(x_{1}, \ldots, x_{m}\right)$ allow us to remove the generators $y \in \mathcal{Y}$ from the presentation. We obtain a finite presentation $\langle\mathcal{X} \mid \tilde{\mathcal{S}}\rangle$ for the group $H$. The finitely many relators $r \in \tilde{\mathcal{S}}$ are normal generators of the kernel $\varphi$. The kernel $\operatorname{ker}(\varphi)$ is $\Phi$-invariant if and only if $r^{\sigma \varphi}={ }_{H} 1$ holds for all $\sigma \in \Phi$. Since $H$ has a decidable word problem and $\Phi$ is finite, the latter problem is recursively recognizable.

Suppose that $\operatorname{ker}(\varphi)$ is $\Phi$-invariant. Then, for each $\sigma \in \Phi \subseteq \operatorname{End}(F)$ there exists an endomorphism $\tilde{\sigma}$ of the free group over $\mathcal{Y}$ so that $\sigma \varphi=\varphi \tilde{\sigma}$ holds; see Appendix B. The map $f: \mathcal{X} \rightarrow H$ induces a group homomorphisms if and only if $\mathcal{Q} \cup \bigcup_{\sigma \in \Phi^{*}} \mathcal{R}^{\sigma} \subseteq \operatorname{ker}(\varphi)$ holds. Since $\mathcal{Q}$ is finite and $H$ has a decidable word problem, the images $q^{\varphi}$, with $q \in \mathcal{Q}$, can be checked one after another. It therefore remains to consider the relations of the form $r^{\sigma \varphi}$. However, it remains to prove that $r^{\varphi}={ }_{H} 1$ holds, as $r^{\sigma \varphi}=r^{\varphi \tilde{\sigma}}$ holds for an endomorphism $\tilde{\sigma} \in\{\tilde{\delta} \mid \delta \in \Phi\}$. Since $\mathcal{R}$ is finite, the images $r^{\varphi}$ can be checked one after another. This yields an algorithm that decides whether or not $f: \mathcal{X} \rightarrow H$ induces a homomorphism $G \rightarrow H$ in the case where $\operatorname{ker}(\varphi)$ is $\Phi$-invariant.

In general, though, we have no solution to the group homomorphism problem for the class of finitely presented group with a decidable word problem. A generalization of the ideas in [70] or Appendix D needs to construct a finite subset $\mathcal{V} \subseteq \Phi^{*}$ for the substitutions of $G$ 's $L$-presentation $\langle\mathcal{X}|\mathcal{Q}| \Phi \mid \mathcal{R}\rangle$ so that, for each $\delta \in \Phi^{*}$, there exists $\sigma \in \mathcal{V}$ with $\operatorname{ker}(\sigma \varphi) \leq \operatorname{ker}(\delta \varphi)$ or, equivalently, if there exists a homomorphism $\pi: \operatorname{im}(\sigma \varphi) \rightarrow \operatorname{im}(\delta \varphi)$ with $\delta \varphi=\sigma \varphi \pi$. In this case, it would be sufficient to verify the finitely many relations $r \in \mathcal{Q} \cup \bigcup_{\sigma \in \mathcal{V}} \mathcal{R}^{\sigma}$.

Question 3.19 Is the group homomorphism problem decidable for the class of finitely presented groups with a solvable word problem? 
$\Gamma_{\text {Chapter }}$

\section{Tietze Transformations for $L$-presentations}

Tietze transformations relate two presentations of the same group: If $\langle\mathcal{X} \mid \mathcal{R}\rangle$ is a presentation for $G, F$ denotes the free group over the alphabet $\mathcal{X}, K$ denotes the normal closure $\langle\mathcal{R}\rangle^{F}$, and $r \in K$ holds, then $\langle\mathcal{X} \mid \mathcal{R} \cup\{r\}\rangle$ is a presentation for $G$. If $\langle\mathcal{X} \mid \mathcal{R}\rangle$ is a presentation for $G, z \notin \mathcal{X}$ is a symbol which is not contained in the alphabet $\mathcal{X}$, and $w_{z} \in F$ is an arbitrary element, then $\left\langle\mathcal{X} \cup\{z\} \mid \mathcal{R} \cup\left\{z^{-1} w_{z}\right\}\right\rangle$ is a presentation for $G$. Both transformations are reversible and are known as Tietze transformations; see, for instance, [95, Chapter II]. Tietze's theorem shows that these transformations (and their inverses) are sufficient to relate two finite presentations of a group by a finite sequence of these Tietze transformations.

For finite $L$-presentations, though, there are further transformations needed because a finite $L$-presentation $\langle\mathcal{X}|\mathcal{Q}| \Phi \mid \mathcal{R}\rangle$ consists of fixed relations $\mathcal{Q}$, iterated relations $\mathcal{R}$, and substitutions $\Phi$. For example, a relation $r \in K$ could be added either as a fixed relation or as an iterated relation. In this chapter, we introduce Tietze transformations for finite $L$-presentations including transformations which allow one to modify the substitutions $\Phi$ of an $L$-presentation. These transformations allow us to prove a version of Tietze's theorem for finitely $L$-presented groups. This theorem shows that two finite $L$-presentations define isomorphic groups if and only if one $L$-presentation can be transformed into the other by a finite number of these transformations. Most of the results in this chapter were generalized in Appendix B in order to consider finitely generated normal subgroups of finitely presented groups.

In practice, Tietze transformations are used to simplify a group presentation. For instance, the group presentations computed with the Reidemeister-Schreier process often contain redundant generators and redundant relations which could be removed from the presentation using Tietze transformations. 


\subsection{Tietze Transformations for finite $L$-presentations}

In this section, we introduce Tietze transformations for finite $L$-presentations; for arbitrary $L$-presentations, we refer to Appendix B below. These transformations generalize the usual Tietze transformations as, for instance, outlined in [95, Chapter II].

Let $G=\langle\mathcal{X}|\mathcal{Q}| \Phi \mid \mathcal{R}\rangle$ be a finitely $L$-presented group. Denote by $F=F(\mathcal{X})$ the free group over the alphabet $\mathcal{X}$ and let $K=\left\langle\mathcal{Q} \cup \bigcup_{\sigma \in \Phi^{*}} \mathcal{R}^{\sigma}\right\rangle^{F}$ be the kernel of the free presentation $\pi: F \rightarrow G$. The kernel $K=\operatorname{ker} \pi$ decomposes into the normal subgroups $Q=\langle\mathcal{Q}\rangle^{F}$ and $R=\left\langle\bigcup_{\sigma \in \Phi^{*}} \mathcal{R}^{\sigma}\right\rangle^{F}$ so that $K=R Q=Q R$ holds. The group $F / R$ is invariantly finitely $L$-presented by $\langle\mathcal{X}|\emptyset| \Phi \mid \mathcal{R}\rangle$. If $r \in K$ is a relation, we obtain the following Tietze transformation:

Proposition 4.1 If $G=\langle\mathcal{X}|\mathcal{Q}| \Phi \mid \mathcal{R}\rangle$ is a finitely L-presented group and $r \in\left\langle\mathcal{Q} \cup \bigcup_{\sigma \in \Phi^{*}} \mathcal{R}^{\sigma}\right\rangle^{F(\mathcal{X})}$ holds, then $\langle\mathcal{X}|\mathcal{Q} \cup\{r\}| \Phi \mid \mathcal{R}\rangle$ is a finite L-presentation for $G$.

Proof. The proof follows immediately with the Tietze transformation that adds a relation $r$ to a group presentation $\left\langle\mathcal{X} \mid \mathcal{Q} \cup \bigcup_{\sigma \in \Phi^{*}} \mathcal{R}^{\sigma}\right\rangle$.

The transformation in Proposition 4.1 is reversible in the sense that we can remove the relation $r$ from the finite $L$-presentation $\langle\mathcal{X}|\mathcal{Q} \cup\{r\}| \Phi \mid \mathcal{R}\rangle$ if and only if $\left\langle\mathcal{Q} \cup\{r\} \cup \bigcup_{\sigma \in \Phi^{*}} \mathcal{R}^{\sigma}\right\rangle^{F(\mathcal{X})}=\left\langle\mathcal{Q} \cup \bigcup_{\sigma \in \Phi^{*}} \mathcal{R}^{\sigma}\right\rangle^{F(\mathcal{X})}$ holds. The following transformations are reversible in a similar sense.

If a finite $L$-presentation is not invariant, there exist elements of the kernel $K=Q R$ that cannot be added as iterated relations without changing the isomorphism type of the group. However, we have the following Tietze transformation:

Proposition 4.2 If $G=\langle\mathcal{X}|\mathcal{Q}| \Phi \mid \mathcal{R}\rangle$ is a finitely L-presented group and $r \in\left\langle\bigcup_{\sigma \in \Phi^{*}} \mathcal{R}^{\sigma}\right\rangle^{F(\mathcal{X})}$ holds, then $\langle\mathcal{X}|\mathcal{Q}| \Phi \mid \mathcal{R} \cup\{r\}\rangle$ is a finite L-presentation for $G$. If $G=\langle\mathcal{X}|\mathcal{Q}| \Phi \mid \mathcal{R}\rangle$ is a finitely L-presented group and $r^{\delta} \in\left\langle\mathcal{Q} \cup \bigcup_{\sigma \in \Phi^{*}} \mathcal{R}^{\sigma}\right\rangle^{F(\mathcal{X})}$ holds for each $\delta \in \Phi^{*}$, then $\langle\mathcal{X}|\mathcal{Q}| \Phi \mid \mathcal{R} \cup\{r\}\rangle$ is a finite L-presentation for $G$.

Proof. The normal subgroup $R=\left\langle\bigcup_{\sigma \in \Phi^{*}} \mathcal{R}^{\sigma}\right\rangle^{F(\mathcal{X})}$ is invariant under each substitution $\sigma \in \Phi^{*}$. More precisely, for each $r \in R$ and $\sigma \in \Phi^{*}$, it holds that $r^{\sigma} \in R$. Therefore, adding the (possibly infinitely many) relations $\left\{r^{\sigma} \mid \sigma \in \Phi^{*}\right\}$ to the group presentation $\left\langle\mathcal{X} \mid \mathcal{Q} \cup \bigcup_{\sigma \in \Phi^{*}} \mathcal{R}^{\sigma}\right\rangle$ does not change the isomorphism type of the group $G$. The second assertion is obvious.

Iterated and fixed relations of a finite $L$-presentation are related by the transformation:

Proposition 4.3 If $G=\langle\mathcal{X}|\mathcal{Q}| \Phi \mid \mathcal{R}\rangle$ is a finitely L-presented group and $r \in \mathcal{R}$ holds, then $\left\langle\mathcal{X}|\mathcal{Q} \cup\{r\}| \Phi \mid(\mathcal{R} \backslash\{r\}) \cup\left\{r^{\psi} \mid \psi \in \Phi\right\}\right\rangle$ is a finite L-presentation for $G$.

Proof. The proof follows immediately from

$$
\mathcal{Q} \cup \bigcup_{\sigma \in \Phi^{*}} \mathcal{R}^{\sigma}=\mathcal{Q} \cup\{r\} \cup \bigcup_{\sigma \in \Phi^{*}}\left((\mathcal{R} \backslash\{r\}) \cup\left\{r^{\psi} \mid \psi \in \Phi\right\}\right)^{\sigma} ;
$$


these are the relations of $G$ 's group presentation.

The following proposition is a consequence of the definition of an invariant $L$-presentation:

Proposition 4.4 If $G=\langle\mathcal{X}|\mathcal{Q}| \Phi \mid \mathcal{R}\rangle$ is an invariantly finitely L-presented group and $q \in \mathcal{Q}$ holds, then $\langle\mathcal{X}|\mathcal{Q} \backslash\{q\}| \Phi \mid \mathcal{R} \cup\{q\}\rangle$ is a finite L-presentation for $G$.

Proof. Since $G$ is invariantly $L$-presented by $\langle\mathcal{X}|\mathcal{Q}| \Phi \mid \mathcal{R}\rangle$, each $\sigma \in \Phi$ induces an endomorphism of the group $G$. Therefore, the images $\left\{q^{\psi} \mid \psi \in \Phi^{*}\right\}$ are relations of the group and thus $\left\langle\mathcal{X} \mid(\mathcal{Q} \backslash\{q\}) \cup \bigcup_{\sigma \in \Phi^{*}}(\mathcal{R} \cup\{q\})^{\sigma}\right\rangle$ is a group presentation for $G$.

The following Tietze transformation adds a generator together with a fixed relation to a finite $L$-presentation:

Proposition 4.5 Let $G=\langle\mathcal{X}|\mathcal{Q}| \Phi \mid \mathcal{R}\rangle$ be a finitely L-presented group, $z \notin \mathcal{X}$, and let $w_{z} \in F(\mathcal{X})$ be given. For each $\sigma \in \Phi$, define an endomorphism of the free group $E$ over the alphabet $\mathcal{X} \cup\{z\}$ that is induced by the map

$$
\tilde{\sigma}:\left\{\begin{aligned}
x & \mapsto x^{\sigma}, \quad \text { for each } x \in \mathcal{X} \\
z & \mapsto g,
\end{aligned}\right.
$$

for an arbitrary $g \in E$. Then $\left\langle\mathcal{X} \cup\{z\}\left|\mathcal{Q} \cup\left\{z^{-1} w_{z}\right\}\right|\{\tilde{\sigma} \mid \sigma \in \Phi\} \mid \mathcal{R}\right\rangle$ is a finite $L$-presentation for $G$.

Proof. Similar to the proof of Proposition 4.1, the proof follows immediately with the Tietze transformation that adds a generator $z \notin \mathcal{X}$ together with a relation $z^{-1} w_{z}$ to the group presentation $\left\langle\mathcal{X} \mid \mathcal{Q} \cup \bigcup_{\sigma \in \Phi^{*}} \mathcal{R}^{\sigma}\right\rangle$. The extended substitution $\tilde{\sigma}$ in Eq. (4.1) can be defined arbitrarily on $z$ because the iterated relations $\mathcal{R}$ do not contain the generator $z$ or its inverse $z^{-1}$ as a subword.

Adding the relation $z^{-1} w_{z}$ as an iterated relation needs the following definition of the extensions $\tilde{\sigma}$ of $\sigma \in \Phi$ :

Proposition 4.6 Let $G=\langle\mathcal{X}|\mathcal{Q}| \Phi \mid \mathcal{R}\rangle$ be a finitely L-presented group, $z \notin \mathcal{X}$, and let $w_{z} \in F(\mathcal{X})$ be given. For each $\sigma \in \Phi$, we define an endomorphism of the free group $E$ over the alphabet $\mathcal{X} \cup\{z\}$ that is induced by the map

$$
\tilde{\sigma}:\left\{\begin{aligned}
x & \mapsto x^{\sigma}, \quad \text { for each } x \in \mathcal{X} \\
z & \mapsto w_{z}^{\sigma} .
\end{aligned}\right.
$$

Then $\left\langle\mathcal{X} \cup\{z\}|\mathcal{Q}|\{\tilde{\sigma} \mid \sigma \in \Phi\} \mid \mathcal{R} \cup\left\{z^{-1} w_{z}\right\}\right\rangle$ is a finite L-presentation for $G$.

Proof. The substitution $\tilde{\sigma}$ in Eq. (4.2) is well-defined as $w_{z} \in F(\mathcal{X})$ and $\sigma \in \operatorname{End}(F(\mathcal{X}))$ hold. Proposition 4.3 yields that

$$
\begin{aligned}
& \left\langle\mathcal{X} \cup\{z\}|\mathcal{Q}|\{\tilde{\sigma} \mid \sigma \in \Phi\} \mid \mathcal{R} \cup\left\{z^{-1} w_{z}\right\}\right\rangle \\
= & \left\langle\mathcal{X} \cup\{z\}\left|\mathcal{Q} \cup\left\{z^{-1} w_{z}\right\}\right|\{\tilde{\sigma} \mid \sigma \in \Phi\} \mid \mathcal{R} \cup\left\{\left(z^{-1} w_{z}\right)^{\tilde{\sigma}} \mid \sigma \in \Phi\right\}\right\rangle .
\end{aligned}
$$


The definition of $\tilde{\sigma}$ in Eq. (4.2) yields that $\left(z^{-1}\right)^{\tilde{\sigma}}=\left(w_{z}^{\sigma}\right)^{-1}$ and $w_{z}^{\tilde{\sigma}}=w_{z}^{\sigma}$. Thus $\left(z^{-1} w_{z}\right)^{\tilde{\sigma}}=1$ holds. In particular, adding the relations $\left\{\left(z^{-1} w_{z}\right)^{\tilde{\sigma}} \mid \sigma \in \Phi\right\}$ to a group presentation does not change the isomorphism type of the group. Finally, Proposition 4.5 yields that

$$
\begin{aligned}
& \left\langle\mathcal{X} \cup\{z\}|\mathcal{Q}|\{\tilde{\sigma} \mid \sigma \in \Phi\} \mid \mathcal{R} \cup\left\{z^{-1} w_{z}\right\}\right\rangle \\
= & \left\langle\mathcal{X} \cup\{z\}\left|\mathcal{Q} \cup\left\{z^{-1} w_{z}\right\}\right|\{\tilde{\sigma} \mid \sigma \in \Phi\} \mid \mathcal{R} \cup\left\{\left(z^{-1} w_{z}\right)^{\tilde{\sigma}} \mid \sigma \in \Phi\right\}\right\rangle \\
= & \left\langle\mathcal{X} \cup\{z\}\left|\mathcal{Q} \cup\left\{z^{-1} w_{z}\right\}\right|\{\tilde{\sigma} \mid \sigma \in \Phi\} \mid \mathcal{R}\right\rangle \\
\cong & \langle\mathcal{X}|\mathcal{Q}| \Phi \mid \mathcal{R}\rangle
\end{aligned}
$$

which proves the assertion of Proposition 4.6

The following version of Proposition 4.3 is a first transformation which modifies the substitutions $\Phi$ of an $L$-presentation:

Proposition 4.7 If $G=\langle\mathcal{X}|\mathcal{Q}| \Phi \mid \mathcal{R}\rangle$ is a finitely L-presented group and $\psi \in \Phi$ holds, then $\left\langle\mathcal{X}|\mathcal{Q}|(\Phi \backslash\{\psi\}) \cup\{\sigma \psi \mid \sigma \in \Phi\} \mid \mathcal{R} \cup \mathcal{R}^{\psi}\right\rangle$ is a finite L-presentation for $G$.

Proof. The proof follows immediately from

$$
\mathcal{Q} \cup \bigcup_{\sigma \in \Phi^{*}} \mathcal{R}^{\sigma}=\mathcal{Q} \cup \bigcup_{\sigma \in \Psi^{*}}\left(\mathcal{R} \cup \mathcal{R}^{\psi}\right)^{\sigma}
$$

where $\Psi=(\Phi \backslash\{\psi\}) \cup\{\sigma \psi \mid \sigma \in \Phi\}$; these are the relations of $G$ 's group presentation.

We also have the following transformation:

Proposition 4.8 Let $G=\langle\mathcal{X}|\mathcal{Q}| \Phi \mid \mathcal{R}\rangle$ be a finitely L-presented group and let $s \in F(\mathcal{X})$ be given. Denote by $\delta_{s}$ the inner automorphism of the free group $F(\mathcal{X})$ that is induced by conjugation with s. Then $\left\langle\mathcal{X}|\mathcal{Q}| \Phi \cup\left\{\delta_{s}\right\} \mid \mathcal{R}\right\rangle,\left\langle\mathcal{X}|\mathcal{Q}| \Phi \backslash\{\sigma\} \cup\left\{\delta_{s} \sigma\right\} \mid \mathcal{R}\right\rangle$, and $\left\langle\mathcal{X}|\mathcal{Q}| \Phi \backslash\{\sigma\} \cup\left\{\sigma \delta_{s}\right\} \mid \mathcal{R}\right\rangle$ are a finite L-presentations for $G$.

Proof. Since each relation of a group presentation can be replaced by a conjugate and $\delta_{x} \sigma=\sigma \delta_{x^{\sigma}}$ holds, for each $\sigma \in \Phi^{*}$, the proof is straightforward.

Recall that the kernel $K=\left\langle\mathcal{Q} \cup \bigcup_{\sigma \in \Phi^{*}} \mathcal{R}^{\sigma}\right\rangle^{F(\mathcal{X})}$ decomposes into the normal subgroups $Q=\langle\mathcal{Q}\rangle^{F(\mathcal{X})}$ and $R=\left\langle\bigcup_{\sigma \in \Phi^{*}} \mathcal{R}^{\sigma}\right\rangle^{F(\mathcal{X})}$ so that $K=Q R$ holds. This decomposition yields the following transformation:

Proposition 4.9 Let $G=\langle\mathcal{X}|\mathcal{Q}| \Phi \mid \mathcal{R}\rangle$ be a finitely L-presented group. If $\psi \in \operatorname{End}(F(\mathcal{X}))$ induces an endomorphism of $F(\mathcal{X}) / R$, then $\langle\mathcal{X}|\mathcal{Q}| \Phi \cup\{\psi\} \mid \mathcal{R}\rangle$ is a finite L-presentation for $G$.

Proof. Suppose that $\psi \in \operatorname{End}(F(\mathcal{X}))$ induces an endomorphism of $F(\mathcal{X}) / R$. Then $R$ is $\psi$-invariant. Thus, each relation $r^{\sigma}$ with $\sigma \in(\Phi \cup\{\psi\})^{*} \backslash \Phi^{*}$ and $r \in \mathcal{R}$ is a relation of the group. Therefore, adding these (possibly infinitely many) relations to the group presentation does not change the isomorphism type of the group.

For an ascending (or invariant) $L$-presentation, we have the following transformation: 
Proposition 4.10 Let $G=\langle\mathcal{X}|\emptyset| \Phi \mid \mathcal{R}\rangle$ be a finitely L-presented group. Then $\langle\mathcal{X}|\emptyset| \Phi \cup\{\psi\} \mid \mathcal{R}\rangle$ is a finite L-presentation for $G$ if and only if $\psi \in \operatorname{End}(F(\mathcal{X}))$ induces an endomorphism of $G=F(\mathcal{X}) / K$.

Proof. If $\psi \in \operatorname{End}(F(\mathcal{X}))$ induces an endomorphism of $F(\mathcal{X}) / K$, Proposition 4.9 shows the first assertion. If, on the other hand, $\langle\mathcal{X}|\emptyset| \Phi \mid \mathcal{R}\rangle$ and $\langle\mathcal{X}|\emptyset| \Phi \cup\{\psi\} \mid \mathcal{R}\rangle$ are finite $L$-presentations for $G, \psi$ induces an endomorphism of $G=F(\mathcal{X}) / K$.

For an invariantly finitely $L$-presented group $G=\langle\mathcal{X}|\mathcal{Q}| \Phi \mid \mathcal{R}\rangle$, every substitution $\sigma \in \Phi$ induces an endomorphism of $G$. However, there are possibly other endomorphisms $\psi \in \operatorname{End}(F(\mathcal{X}))$ that induce the same endomorphism of $G$. The following proposition allows us to replace a substitution $\sigma \in \Phi$ by an endomorphism $\psi \in \operatorname{End}(F(\mathcal{X}))$ :

Proposition 4.11 Let $G=\langle\mathcal{X}|\mathcal{Q}| \Phi \mid \mathcal{R}\rangle$ be a finitely L-presented group, $r \in\left\langle\bigcup_{\varphi \in \Phi^{*}} \mathcal{R}^{\varphi}\right\rangle^{F(\mathcal{X})}, z \in \mathcal{X}$, and let $\sigma \in \Phi$ be given. Define an endomorphism $\tilde{\sigma}$ of the free group $F(\mathcal{X})$ over the alphabet $\mathcal{X}$ that is induced by the map

$$
\tilde{\sigma}:\left\{\begin{aligned}
z & \mapsto z^{\sigma} r \\
x & \mapsto x^{\sigma}, \quad \text { for each } x \in \mathcal{X} \backslash\{z\} .
\end{aligned}\right.
$$

Then $\langle\mathcal{X}|\mathcal{Q}|(\Phi \backslash\{\sigma\}) \cup\{\tilde{\sigma}\} \mid \mathcal{R} \cup\{r\}\rangle$ is a finite L-presentation for $G$.

Proof. We decompose the kernel $K=\left\langle\mathcal{Q} \bigcup_{\varphi \in \Phi^{*}} \mathcal{R}^{\varphi}\right\rangle^{F(\mathcal{X})}$ of the free presentation $\pi: F(\mathcal{X}) \rightarrow G$ into the normal subgroups $Q=\langle\mathcal{Q}\rangle^{F(\mathcal{X})}$ and $R=\left\langle\bigcup_{\varphi \in \Phi^{*}} \mathcal{R}^{\varphi}\right\rangle^{F(\mathcal{X})}$ so that $K=Q R=R Q$ holds. Since $r \in\left\langle\bigcup_{\varphi \in \Phi^{*}} \mathcal{R}^{\varphi}\right\rangle^{F(\mathcal{X})}$ holds, Proposition 4.2 yields that

$$
G=\langle\mathcal{X}|\mathcal{Q}| \Phi \mid \mathcal{R}\rangle=\langle\mathcal{X}|\mathcal{Q}| \Phi \mid \mathcal{R} \cup\{r\}\rangle
$$

and $R=\left\langle\bigcup_{\varphi \in \Phi^{*}}(\mathcal{R} \cup\{r\})^{\varphi}\right\rangle^{F(\mathcal{X})}$. Write $\Psi=(\Phi \cup\{\tilde{\sigma}\}) \backslash\{\sigma\}$. We prove that the normal subgroups $R=\left\langle\bigcup_{\varphi \in \Phi^{*}}(\mathcal{R} \cup\{r\})^{\varphi}\right\rangle^{F(\mathcal{X})}$ and $\tilde{R}=\left\langle\bigcup_{\varphi \in \Psi^{*}}(\mathcal{R} \cup\{r\})^{\varphi}\right\rangle^{F(\mathcal{X})}$ coincide. For this purpose, we prove that, for each $\tilde{\delta} \in \Psi^{*}$ and $g \in F(\mathcal{X})$, there exist $\delta \in \Phi^{*}$ and $h \in L=\left\langle r^{\varphi} \mid \varphi \in \Phi^{*}\right\rangle^{F(\mathcal{X})}$ so that $g^{\tilde{\delta}}=g^{\delta} \cdot h$ holds. By construction, we have that $L \subseteq R$. By symmetry, as we have both $z^{\tilde{\sigma}}=z^{\sigma} r$ and $z^{\tilde{\sigma}} r^{-1}=z^{\sigma}$, the same arguments will show that, for each $\delta \in \Phi^{*}$ and $g \in F(\mathcal{X})$, there exist $\tilde{\delta} \in \Psi^{*}$ and $h \in \tilde{L}=\left\langle r^{\varphi} \mid \varphi \in \Psi^{*}\right\rangle^{F(\mathcal{X})}$ so that $g^{\delta}=g^{\tilde{\delta}} \cdot h$. If we have proved this, each normal generator $s^{\tilde{\varphi}} \in \tilde{R}$, with $s \in \mathcal{R} \cup\{r\}$ and $\tilde{\varphi} \in \Psi^{*}$, can be written as $s^{\tilde{\varphi}}=s^{\varphi} \cdot h$ with $\varphi \in \Phi^{*}$ and $h \in L \subseteq R$. Thus $s^{\tilde{\varphi}}=s^{\varphi} \cdot h \in R$ and $\tilde{R} \subseteq R$. By symmetry, we would also obtain $R \subseteq \tilde{R}$.

Write $\mathcal{X}=\left\{x_{1}, \ldots, x_{m}, z\right\}$. Each $g \in F(\mathcal{X})$ is represented by a word $w_{g}\left(x_{1}, \ldots, x_{m}, z\right)$ over the generators $\mathcal{X}$. Let $\tilde{\delta} \in \Psi^{*}$ and $g \in F(\mathcal{X})$ be given. We prove the assertion by induction on $n=\|\tilde{\delta}\|$. If $n=1$, then $\tilde{\delta} \in \Psi$ holds. If $\tilde{\delta} \neq \tilde{\sigma}$ holds, then $\tilde{\delta} \in \Phi$. Thus $g^{\tilde{\delta}}=g^{\delta}$ for some $\delta \in \Phi$. Otherwise, if $\tilde{\delta}=\tilde{\sigma}$, we obtain that

$$
g^{\tilde{\delta}}=g^{\tilde{\sigma}}=w_{g}\left(x_{1}, \ldots, x_{m}, z\right)^{\tilde{\sigma}}=w_{g}\left(x_{1}^{\tilde{\sigma}}, \ldots, x_{m}^{\tilde{\sigma}}, z^{\tilde{\sigma}}\right)=w_{g}\left(x_{1}^{\sigma}, \ldots, x_{m}^{\sigma}, z^{\sigma} r\right) .
$$


Conjugation in the free group $F(\mathcal{X})$ yields that the word $w_{g}\left(x_{1}^{\sigma}, \ldots, x_{m}^{\sigma}, z^{\sigma} r\right)$ can be written as $w_{g}\left(x_{1}^{\sigma}, \ldots, x_{m}^{\sigma}, z^{\sigma}\right) \cdot h$ for some $h \in\langle r\rangle^{F(\mathcal{X})} \subseteq L$. Thus $g^{\tilde{\sigma}}=g^{\sigma} \cdot h$ holds for $\sigma \in \Phi$ and some $h \in L$.

For a positive integer $n>1$, we assume that every image $g^{\tilde{\delta}}$, with $g \in F(\mathcal{X}), \tilde{\delta} \in \Psi^{*}$, and $\|\tilde{\delta}\|=n$, can be written as $g^{\delta} \cdot h$ for $\delta \in \Phi^{*}$ and $h \in L$. Let $g \in F(\mathcal{X})$ and $\tilde{\gamma} \in \Psi^{*}$, with $\|\tilde{\gamma}\|=n+1$, be given. Then there exist $\tilde{\omega} \in \Psi$ and $\tilde{\delta} \in \Psi^{*}$, with $\|\tilde{\delta}\|=n$, so that $\tilde{\gamma}=\tilde{\delta} \tilde{\omega}$ holds. By our assumption we have that $g^{\tilde{\gamma}}=g^{\tilde{\delta} \tilde{\omega}}=\left(g^{\delta} \cdot h\right)^{\tilde{\omega}}$ for some $\delta \in \Phi^{*}$ and $h \in L$. If $\tilde{\omega} \neq \tilde{\sigma}$, then $\tilde{\omega} \in \Phi$ and thus $\delta \tilde{\omega} \in \Phi^{*}$. Moreover, by construction, the normal subgroups $L=\left\langle r^{\varphi} \mid \varphi \in \Phi^{*}\right\rangle^{F(\mathcal{X})}$ and $\tilde{L}=\left\langle r^{\tilde{\varphi}} \mid \tilde{\varphi} \in \Psi^{*}\right\rangle^{F(\mathcal{X})}$ are $\Phi^{*}$ - and $\Psi^{*}$-invariant, respectively. Thus $h^{\tilde{\omega}} \in L$ if $\tilde{\omega} \neq \tilde{\sigma}$. Therefore, the image $g^{\tilde{\gamma}}$ can be written as $g^{\tilde{\gamma}}=\left(g^{\delta} \cdot h\right)^{\tilde{\omega}}=g^{\delta \tilde{\omega}} \cdot h^{\tilde{\omega}}$ with $\delta \tilde{\omega} \in \Phi^{*}$ and $h^{\tilde{\omega}} \in L$. It therefore suffices to consider the case $\tilde{\omega}=\tilde{\sigma}$. The elements $g^{\delta}, h \in F(\mathcal{X})$ are represented by words $w_{g^{\delta}}\left(x_{1}, \ldots, x_{m}, z\right)$ and $w_{h}\left(x_{1}, \ldots, x_{m}, z\right)$, respectively. Again, conjugation in the free group $F(\mathcal{X})$ yields that $g^{\tilde{\gamma}}=\left(g^{\delta} h\right)^{\tilde{\sigma}}=\left(g^{\delta \sigma} \cdot a\right) \cdot\left(h^{\sigma} \cdot b\right)$ for some $a, b \in\langle r\rangle^{F(\mathcal{X})} \subseteq L$. Hence, the image $g^{\tilde{\gamma}}$ can be written as $g^{\delta \sigma} \cdot h^{\prime}$ for $\delta \sigma \in \Phi^{*}$ and $h^{\prime}=a h^{\sigma} b \in L$. By induction on $n$, it follows that each image $g^{\tilde{\delta}}$, with $g \in F(\mathcal{X})$ and $\tilde{\delta} \in \Psi^{*}$, has the form $g^{\tilde{\delta}}=g^{\delta} \cdot h$ for some $\delta \in \Phi^{*}$ and $h \in L$. By symmetry, the same arguments above show that each image $g^{\delta}$, with $g \in F(\mathcal{X})$ and $\delta \in \Phi^{*}$, can be written as $g^{\delta}=g^{\tilde{\delta}} \cdot h$ for $\tilde{\delta} \in \Psi^{*}$ and $h \in \tilde{L}$. This finishes our proof of Proposition 4.11.

Note that Proposition 4.11 allows us to replace a substitution $\sigma \in \Phi$ of an $L$-presentation $\langle\mathcal{X}|\mathcal{Q}| \Phi \mid \mathcal{R}\rangle$ by an endomorphism $\psi \in \operatorname{End}(F(\mathcal{X}))$ of the free group $F(\mathcal{X})$ that induces the same endomorphism on group. More precisely, we obtain the following

Corollary 4.12 Let $G$ be finitely L-presented by $\langle\mathcal{X}|\mathcal{Q}| \Phi \mid \mathcal{R}\rangle$. Then every substitution $\sigma \in \Phi$ induces an endomorphism of the invariantly finitely L-presented group $H=$ $\langle\mathcal{X}|\emptyset| \Phi \mid \mathcal{R}\rangle$. If $\psi \in \operatorname{End}(F(\mathcal{X}))$ induces the same endomorphism on $H$, there exists a finite set $\mathcal{S} \subseteq F(\mathcal{X})$ so that $G$ is finitely L-presented by $\langle\mathcal{X}|\mathcal{Q}|(\Phi \backslash\{\sigma\}) \cup\{\psi\} \mid \mathcal{R} \cup \mathcal{S}\rangle$.

Proof. If $\psi$ and $\sigma$ induce the same endomorphism of $H$ there exists, for each $z \in \mathcal{X}, r_{z} \in$ $\left\langle\bigcup_{\varphi \in \Phi^{*}} \mathcal{R}^{\varphi}\right\rangle^{F(\mathcal{X})}$ so that $z^{\psi}=z^{\sigma} r_{z}$ holds. Write $\mathcal{S}=\left\{r_{z} \mid z \in \mathcal{X}\right\}$. By Proposition 4.11, a finite $L$-presentation for $G$ is given by $\langle\mathcal{X}|\mathcal{Q}|(\Phi \backslash\{\sigma\}) \cup\{\psi\} \mid \mathcal{R} \cup \mathcal{S}\rangle$.

For our proof of a version of Tietze's theorem for finitely $L$-presented groups we consider the following transformations:

(i) adds or removes a fixed relation (Proposition 4.1),

(ii) adds or removes an iterated relation (Proposition 4.2),

(iii) adds or removes a substitution (Proposition 4.9),

(iv) adds or removes a generator together with a fixed relation (Proposition 4.5), or,

(v) adds or removes a generator together with an iterated relation (Proposition 4.6). 


\subsection{Tietze's Theorem for Finite $L$-Presentations}

We prove a version of Tietze's theorem for finite $L$-presentations:

Theorem 4.13 Two finite L-presentations define isomorphic groups if and only if it is possible to pass from one L-presentation to the other by a finite sequence of Tietze transformations.

Proof. We use similar ideas as in the proof of Tietze's theorem in [95, Chapter II]: As each Tietze transformation does not change the isomorphism type of the group, two finite $L$-presentations define isomorphic groups if they can be transformed into each other by a sequence of Tietze transformations. In order to prove Theorem 4.13, it therefore suffices to prove that two finite $L$-presentations which define isomorphic groups can be transformed into each other by a finite sequence of Tietze transformations. For this purpose, consider two finite $L$-presentations $\left\langle\mathcal{X}_{1}\left|\mathcal{Q}_{1}\right| \Phi_{1} \mid \mathcal{R}_{1}\right\rangle$ and $\left\langle\mathcal{X}_{2}\left|\mathcal{Q}_{2}\right| \Phi_{2} \mid \mathcal{R}_{2}\right\rangle$ of a group $G$. Suppose that $\mathcal{X}_{1} \cap \mathcal{X}_{2}=\emptyset$ holds. For $1 \leq i \leq 2$, denote by $F_{i}$ the free group over the alphabet $\mathcal{X}_{i}$ and let $\pi_{i}: F_{i} \rightarrow G$ be the free presentation with kernel $\operatorname{ker}\left(\pi_{i}\right)=\left\langle\mathcal{Q}_{i} \cup \bigcup_{\sigma \in \Phi_{i}^{*}} \mathcal{R}_{i}^{\sigma}\right\rangle^{F_{i}}$. For each $x \in \mathcal{X}_{1}$, we choose $w_{x} \in F_{2}$ with $x^{\pi_{1}}=w_{x}^{\pi_{2}}$; i.e., $w_{x} \in F_{2}$ is a $\pi_{2}$-preimage of $x^{\pi_{1}} \in G$. For each $z \in \mathcal{X}_{2}$, choose $w_{z} \in F_{1}$ with $z^{\pi_{2}}=w_{z}^{\pi_{1}}$. Define the finite subsets $S_{1}$ and $S_{2}$ of the free group $F$ over $\mathcal{X}_{1} \cup \mathcal{X}_{2}$ by

$$
S_{1}=\left\{x^{-1} w_{x} \mid x \in \mathcal{X}_{1}\right\} \quad \text { and } \quad S_{2}=\left\{z^{-1} w_{z} \mid z \in \mathcal{X}_{2}\right\}
$$

By Proposition 4.5, we can add the generator $z \in \mathcal{X}_{2}$ together with the fixed relation $z^{-1} w_{z} \in S_{2}$ if we extend each substitution $\sigma \in \Phi_{1}$ to the free group over $\mathcal{X}_{1} \cup\{z\}$ by defining $x \mapsto x^{\sigma}$, for $x \in \mathcal{X}_{1}$, and $z \mapsto z$, otherwise. Therefore, Proposition 4.5 allows us to add the generators $z \in \mathcal{X}_{2}$ together with a fixed relation $z^{-1} w_{z} \in S_{2}$ to the finite $L$-presentation $\left\langle\mathcal{X}_{1}\left|\mathcal{Q}_{1}\right| \Phi_{1} \mid \mathcal{R}_{1}\right\rangle$. More precisely, if $F$ denotes the free group over $\mathcal{X}_{1} \cup \mathcal{X}_{2}$ and, for each $\sigma \in \Phi_{1}, \tilde{\sigma}$ denotes the endomorphism of the free group $F$ that is induced by the map

$$
\tilde{\sigma}: F \rightarrow F, \quad\left\{\begin{array}{lll}
x & \mapsto x^{\sigma}, & \text { if } x \in \mathcal{X}_{1}, \\
z & \mapsto z, & \text { if } z \in \mathcal{X}_{2}
\end{array}\right.
$$

Proposition 4.5 yields that

$$
G \cong\left\langle\mathcal{X}_{1} \cup \mathcal{X}_{2}\left|\mathcal{Q}_{1} \cup S_{2}\right|\{\tilde{\sigma}\}_{\sigma \in \Phi_{1}} \mid \mathcal{R}_{1}\right\rangle
$$

The natural homomorphisms $\pi_{1}: F_{1} \rightarrow G$ and $\pi_{2}: F_{2} \rightarrow G$ extend to the natural homomorphism $\pi: F \rightarrow G$ that is induced by the map

$$
\pi:\left\{\begin{array}{rll}
x & \mapsto x^{\pi_{1}}, & \text { if } x \in \mathcal{X}_{1}, \\
z & \mapsto z^{\pi_{2}}, & \text { if } z \in \mathcal{X}_{2} .
\end{array}\right.
$$

For $x \in \mathcal{X}_{1}$ and $x^{-1} w_{x} \in S_{1}$, we have $x^{\pi}=x^{\pi_{1}}=w_{x}^{\pi_{2}}=w_{x}^{\pi}$ and thus $x^{-1} w_{x} \in \operatorname{ker}(\pi)$. For each $r \in \mathcal{Q}_{2}$, we have $r^{\pi}=r^{\pi_{2}}=1$. Thus $r \in \mathcal{Q}_{2}$. By Proposition 4.1, we can 
therefore add $\mathcal{Q}_{2} \cup S_{1}$ as fixed relations to the $L$-presentation in Eq. (4.5). We obtain the finite $L$-presentation

$$
G \cong\left\langle\mathcal{X}_{1} \cup \mathcal{X}_{2}\left|\mathcal{Q}_{1} \cup \mathcal{Q}_{2} \cup S_{1} \cup S_{2}\right|\{\tilde{\sigma}\}_{\sigma \in \Phi_{1}} \mid \mathcal{R}_{1}\right\rangle .
$$

For each $\sigma \in \Phi_{2}$, define an endomorphism of the free group $F$ that is induced by the map

$$
\tilde{\sigma}: F \rightarrow F,\left\{\begin{array}{llll}
x & \mapsto x, & \text { if } x \in \mathcal{X}_{1} \\
z & \mapsto z^{\sigma}, & \text { if } x \in \mathcal{X}_{2}
\end{array}\right.
$$

Then $\tilde{\sigma}$ acts trivially on the generators $\mathcal{X}_{1}$ and therefore, it leaves the normal subgroup $\left\langle\bigcup_{\delta \in \Phi_{1}^{*}} \mathcal{R}_{1}^{\tilde{\delta}}\right\rangle^{F}$ invariant. By Proposition 4.9, we can add the substitutions $\left\{\tilde{\sigma} \mid \sigma \in \Phi_{2}\right\}$ to the $L$-presentation in Eq. (4.6) and we obtain the $L$-presentation

$$
G \cong\left\langle\mathcal{X}_{1} \cup \mathcal{X}_{2}\left|\mathcal{Q}_{1} \cup \mathcal{Q}_{2} \cup S_{1} \cup S_{2}\right|\{\tilde{\sigma}\}_{\sigma \in \Phi_{1} \cup \Phi_{2}} \mid \mathcal{R}_{1}\right\rangle
$$

It remains to consider the relations $\mathcal{R}_{2}$ from the finite $L$-presentation $\left\langle\mathcal{X}_{2}\left|\mathcal{Q}_{2}\right| \Phi_{2} \mid \mathcal{R}_{2}\right\rangle$ and it suffices to show that we can add these relations $r \in \mathcal{R}_{2}$ as iterated relations to the $L$-presentation in Eq. (4.8). For this purpose, we need to prove that, for each $\delta \in\left\{\tilde{\sigma} \mid \sigma \in \Phi_{1} \cup \Phi_{2}\right\}^{*}$, the image $r^{\delta}$ is a relation of the group; i.e., $r^{\delta}$ is contained in the kernel $\operatorname{ker}(\pi)$. Let $\delta \in\left\{\tilde{\sigma} \mid \sigma \in \Phi_{1} \cup \Phi_{2}\right\}^{*}$ be given. We can write $\delta=\tilde{\sigma}_{i_{1}} \tilde{\sigma}_{j_{1}} \cdots \tilde{\sigma}_{i_{n}} \tilde{\sigma}_{j_{n}}$ with each $\sigma_{i_{\ell}} \in\{\varepsilon\} \cup \Phi_{1}$ and $\sigma_{j_{\ell}} \in\{\varepsilon\} \cup \Phi_{2}$. Since each $\tilde{\sigma}_{i_{\ell}}$, with $\sigma_{i_{\ell}} \in \Phi_{1}$, acts trivially on the generators $\mathcal{X}_{2}$ and each $\tilde{\sigma}_{j_{\ell}}$, with $\sigma_{j_{\ell}} \in \Phi_{2}$, leaves the subgroup which is generated by $\mathcal{X}_{2}$ invariant, we obtain that $r^{\delta}=r^{\tilde{\sigma}_{i_{1}}} \tilde{\sigma}_{j_{1}} \cdots \tilde{\sigma}_{i_{n}} \tilde{\sigma}_{j_{n}}=r^{\tilde{\sigma}_{j_{1}} \cdots \tilde{\sigma}_{j_{n}}}$. This yields that $\left(r^{\delta}\right)^{\pi}=\left(r^{\tilde{\sigma}_{j_{1}} \cdots \tilde{\sigma}_{j_{n}}}\right)^{\pi}=\left(r^{\sigma_{j_{1}} \cdots \sigma_{j_{n}}}\right)^{\pi_{2}}=1$ holds. Thus, by Proposition 4.2, we can add the relations $\mathcal{R}_{2}$ as iterated relations to the $L$-presentation in Eq. (4.8) and we obtain the $L$-presentation

$$
G \cong\left\langle\mathcal{X}_{1} \cup \mathcal{X}_{2}\left|\mathcal{Q}_{1} \cup \mathcal{Q}_{2} \cup S_{1} \cup S_{2}\right|\{\tilde{\sigma}\}_{\sigma \in \Phi_{1} \cup \Phi_{2}} \mid \mathcal{R}_{1} \cup \mathcal{R}_{2}\right\rangle
$$

Since the $L$-presentations $\left\langle\mathcal{X}_{1}\left|\mathcal{Q}_{1}\right| \Phi_{1} \mid \mathcal{R}_{1}\right\rangle$ and $\left\langle\mathcal{X}_{2}\left|\mathcal{Q}_{2}\right| \Phi_{2} \mid \mathcal{R}_{2}\right\rangle$ were finite, we have applied finitely many Tietze transformations to obtain the finite $L$-presentation in Eq. (4.9) from the finite $L$-presentation $\left\langle\mathcal{X}_{1}\left|\mathcal{Q}_{1}\right| \Phi_{1} \mid \mathcal{R}_{1}\right\rangle$. By symmetry, though, we would also obtain the finite $L$-presentation in Eq. (4.9) from the $L$-presentation $\left\langle\mathcal{X}_{2}\left|\mathcal{Q}_{2}\right| \Phi_{2} \mid \mathcal{R}_{2}\right\rangle$. As each Tietze transformation is reversible, we can therefore transform the finite $L$-presentation in Eq.(4.9) to the finite $L$-presentation $\left\langle\mathcal{X}_{2}\left|\mathcal{Q}_{2}\right| \Phi_{2} \mid \mathcal{R}_{2}\right\rangle$. This shows that both finite $L$-presentations of $G$ could be transformed into each other by a finite sequence of Tietze transformations.

Similar to finite presentations, Theorem 4.13 does not solve the isomorphism problem for finitely $L$-presented groups as there is no effective procedure for choosing the sets $S_{1}$ and $S_{2}$. In particular, there exists no algorithm which allows one to prove that two finite $L$-presentations define non-isomorphic groups. In Appendix B, we use the Tietze transformations introduced above to prove

Theorem 4.14 (Bartholdi [6]) Being invariantly finitely L-presented is an abstract property of a group which does not depend on the generating set.

This theorem was already posed in [6, Proposition 2.2]. However, its proof contains a gap which we could address with the help of our Tietze transformations above. 


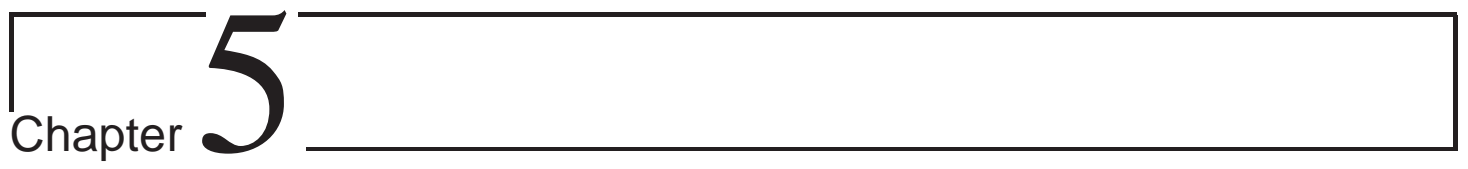

\section{Finitely Based Infinite Rewriting Systems}

The group $\mathfrak{G}$ constructed by Grigorchuk in [53] can be realized as a subgroup of the automorphism group of the rooted binary tree. As it acts faithfully on the tree, the word problem of the group $\mathfrak{G}$ is decidable. Alternatively, the group $\mathfrak{G}$ can be defined by the invariant finite $L$-presentation

$$
\left.\mathfrak{G}=\langle\{a, b, c, d\}|\left\{a^{2}, b^{2}, c^{2}, d^{2}, b c d\right\} \cup \bigcup_{i \geq 0}\left\{(a d)^{4},(\text { adacac })^{4}\right\}^{\sigma^{i}}\right\rangle,
$$

where $\sigma$ is induced by the map $a \mapsto a c a, b \mapsto d, c \mapsto b$, and $d \mapsto c$; see Theorem 2.14, The substitution $\sigma$ induces a monomorphism of the group $\mathfrak{G}$ [55]. In this chapter, we develop a method for computing canonical forms for elements of an invariantly finitely $L$-presented group. For finite presentations, the Knuth-Bendix completion [86] computes a confluent rewriting system if it terminates. A confluent rewriting system allows to compute canonical forms of an element in the finitely presented groups and therefore, it allows one to solve the word problem. We generalize the Knuth-Bendix completion for finite presentations to invariant finite $L$-presentations. Since the group $\mathfrak{G}$ is not finitely presentable [56], there will not exist a finite rewriting system. Therefore, our procedure attempts to compute an infinite rewriting system whose rewriting rules incorporate the substitution $\sigma$. More precisely, we attempt to construct a rewriting system whose (possibly infinitely many) rewriting rules are given by a finite set of rewriting rules and their images under the substitutions.

A rewriting system consists of a set of objects and a set of rewriting rules that describe the transformations of the objects. For an invariantly finitely $L$-presented group $G=\langle\mathcal{X}|\mathcal{Q}| \Phi \mid \mathcal{R}\rangle$, we establish a rewriting system with objects

$$
\mathcal{S}\left(\mathcal{X}^{ \pm}, \Phi\right)=\left\{\left(x_{1}, \sigma_{1}\right) \cdots\left(x_{n}, \sigma_{n}\right) \mid x_{i} \in \mathcal{X}^{ \pm}, \sigma_{i} \in \Phi^{*}, 1 \leq i \leq n\right\}
$$

where $\mathcal{X}^{ \pm}$are the monoid generators of the finitely $L$-presented group $G$ and $\Phi^{*}$ denotes the free monoid that is generated by the finite set $\Phi$. The objects $\mathcal{S}\left(\mathcal{X}^{ \pm}, \Phi\right)$ are elements of the free monoid over the Cartesian product $\mathcal{X}^{ \pm} \times \Phi^{*}$. For further details on rewriting systems, we refer to [23, 129]. 
Computer experiments with our Knuth-Bendix completion do not terminate and thus do not allow to compute a confluent infinite rewriting system for the group $\mathfrak{G}$ as well as for the Basilica group and the wreath products $\mathbb{Z}_{2} \prec \mathbb{Z}$ or $\mathbb{Z} \imath \mathbb{Z}$. Therefore, it is not clear whether or not these groups admit a 'finitely based infinite rewriting system' as would have been constructed by our Knuth-Bendix completion.

In Section 5.1, we recall the notion of a rewriting system as, for instance, introduced in [129. Then, in Section 5.2, we construct a monoid presentation for an invariantly finitely $L$-presented group with objects $S\left(\mathcal{X}^{ \pm}, \Phi\right)$. For constructing a rewriting system as in [129] we further need a reduction ordering on the elements $\mathcal{S}\left(\mathcal{X}^{ \pm}, \Phi\right)$. This reduction ordering is constructed in Section 5.3. Then, in Section 5.4 we prove the fundamental Critical Pair Lemma for the new type of 'finitely based infinite rewriting systems'. This lemma allows us to decide whether or not a given rewriting system is confluent. Moreover, it yields a Knuth-Bendix completion in Section 5.5, Finally, in Section [5.6, we demonstrate the application of our Knuth-Bendix algorithm to a fairly easy invariant finite $L$-presentation.

In the following, a finite $L$-presentation is always considered to be invariant if not otherwise stated. In particular, an invariant finite $L$-presentation $\langle\mathcal{X}|\mathcal{Q}| \Phi \mid \mathcal{R}\rangle$ can always be chosen so that $\mathcal{Q}=\emptyset$ holds. Our generalization of the Knuth-Bendix completion algorithm follows [129, Chapter 2].

\subsection{Preliminaries}

In this section, we introduce the notion of a rewriting system as in [129]. For this purpose, let $\mathcal{X}$ be a (possibly infinite) alphabet and denote by $\mathcal{X}^{*}$ the free monoid over $\mathcal{X}$. From a set of binary relations $\mathcal{R} \subseteq \mathcal{X}^{*} \times \mathcal{X}^{*}$, we obtain a monoid congruence $\sim$ on $\mathcal{X}^{*}$ as follows: Denote by $\widehat{\mathcal{R}}$ the symmetric closure of $\mathcal{R}$. For $U, V \in \mathcal{X}^{*}$, we define $U \sim V$ if there exists $A, B, P, Q \in \mathcal{X}^{*}$ so that $U=A P B, V=A Q B$, and $(P, Q) \in \widehat{\mathcal{R}}$ hold. The reflexive and transitive closure of $\sim$ is the monoid congruence $\sim_{\mathcal{R}}$ generated by $\mathcal{R}$. The monoid presentation

$$
\langle\mathcal{X} \mid\{P=Q \mid(P, Q) \in \mathcal{R}\}\rangle
$$

defines the monoid $\mathcal{X}^{*} / \sim_{\mathcal{R}}$ of equivalence classes of $\sim_{\mathcal{R}}$. In the following, we often will avoid the reference to $\mathcal{R}$ if it is clear from the context which generating set we choose.

We can endow the free monoid $\mathcal{X}^{*}$ with a reduction ordering $\prec$ : A reduction ordering is a total and translation invariant well-ordering; i.e., an ordering $\prec$ is translation invariant if $P \succ Q$ implies $A P B \succ A Q B$ for each $A, B \in \mathcal{X}^{*}$. Since $\sim_{\mathcal{R}}$ is symmetric and $\prec$ is a total ordering, we can assume that $(P, Q) \in \mathcal{R}$ always implies $P \succ Q$ (otherwise we can replace $(P, Q) \in \mathcal{R}$ by $(Q, P)$ if $Q \succ P$ or we can omit $(P, Q) \in \mathcal{R}$ if $P=Q$ holds. Obviously this does not change the monoid congruence $\sim_{\mathcal{R}}$ ). The monoid presentation in Eq. (5.3) yields the rewriting system

$$
\mathcal{T}=\{P \rightarrow Q \mid P \succ Q,(P, Q) \in \mathcal{R} \text { or }(Q, P) \in \mathcal{R}\} .
$$

For $U, V \in \mathcal{X}^{*}$, define, if possible, a one-step reduction $U \rightarrow_{\mathcal{T}} V$ with respect to $\mathcal{T}$ if there exist $A, B, P, Q \in \mathcal{X}^{*}$ so that $U=A P B, V=A Q B$, and $P \rightarrow Q$ is a rewriting rule in $\mathcal{T}$. 
Denote by $\rightarrow_{\mathcal{T}}^{*}$ the reflexive and transitive closure of $\rightarrow_{\mathcal{T}}$. Clearly, a one-step reduction $U \rightarrow_{\mathcal{T}} V$ or, in general, a reduction $U \rightarrow_{\mathcal{T}}^{*} V$ always implies $U \sim V$. In the following, we do not distinguish between the rewriting rule $U \rightarrow \mathcal{T} V$ from $\mathcal{T}$ and the relation $(U, V)$ from the monoid presentation. In particular, the rewriting rules of the rewriting system $\mathcal{T}$ always generate a monoid congruence $\sim_{\mathcal{T}}$.

A rewriting system $\mathcal{T}$ allows us to transform the elements of $\mathcal{X}^{*}$ with respect to the reduction ordering $\prec$ and the monoid congruence $\sim_{\mathcal{T}}$, see [129]: Let $\mathcal{N}$ be the ideal that is generated as an ideal by the left-sides $\left\{P \mid P \rightarrow_{\mathcal{T}} Q \in \mathcal{T}\right\}$. If $U_{0} \in \mathcal{N}$, there exist $A, B, P, Q \in \mathcal{X}^{*}$ with $U_{0}=A P B$ and $P \rightarrow \mathcal{T} Q \in \mathcal{T}$. Then $U_{1}=A Q B$ satisfies $U_{0} \succ U_{1}$, $U_{0} \rightarrow \mathcal{T} U_{1}$, and $U_{0} \sim_{\mathcal{T}} U_{1}$. If $U_{1} \in \mathcal{N}$ holds, we can repeat this process and obtain $U_{2} \in \mathcal{X}^{*}$ with $U_{1} \succ U_{2}, U_{1} \rightarrow \mathcal{T} U_{2}$, and $U_{1} \sim_{\mathcal{T}} U_{2}$. This yields a descending sequence $U_{0} \succ U_{1} \succ U_{2} \succ \ldots$ in the well-ordered monoid $\mathcal{X}^{*}$. Thus the sequence $U_{0}, U_{1}, U_{2}, \ldots$ eventually terminates with an element $U_{n} \in \mathcal{X}^{*} \backslash \mathcal{N}$ that satisfies $U_{0} \sim_{\mathcal{T}} U_{n}, U_{0} \rightarrow_{\mathcal{T}}^{*} U_{n}$, and $U_{0} \succ U_{n}$. The elements $U_{n} \in \mathcal{X}^{*} \backslash \mathcal{N}$ cannot be transformed any further. Therefore, $U_{n}$ is irreducible with respect to $\mathcal{T}$. The above process of transforming the element $U_{0} \in \mathcal{X}^{*}$ with the rewriting system $\mathcal{T}$ is called rewriting. Each rewriting system allows us to rewrite each element to an irreducible one. The rewriting system depends on the reduction ordering $\prec$.

Rewriting is non-deterministic because there are possibly different choices of one-step reductions $U_{i} \rightarrow_{\mathcal{T}} U_{i+1}$ within a rewriting process. Therefore, for a rewriting system $\mathcal{T}$, we have the following notations:

- An element $W \in \mathcal{X}^{*}$ is locally confluent, if, for each $U, V \in \mathcal{X}^{*}$ with $W \rightarrow_{\mathcal{T}} U$ and $W \rightarrow \mathcal{T} V$, there exists $Z \in \mathcal{X}^{*}$ with $U \rightarrow_{\mathcal{T}}^{*} Z$ and $V \rightarrow_{\mathcal{T}}^{*} Z$.

- An element $W \in \mathcal{X}^{*}$ is confluent, if, for each $U, V \in \mathcal{X}^{*}$ with $W \rightarrow_{\mathcal{T}}^{*} U$ and $W \rightarrow_{\mathcal{T}}^{*} V$, there exists $Z \in \mathcal{X}^{*}$ with $U \rightarrow_{\mathcal{T}}^{*} Z$ and $V \rightarrow_{\mathcal{T}}^{*} Z$.

Clearly, an element $W \in \mathcal{X}^{*}$ is locally confluent whenever it is confluent. In general, though, local confluence does not imply confluence [23, Section 1.2]. A rewriting system $\mathcal{T}$ is (locally) confluent if and only if all elements $W \in \mathcal{X}^{*}$ are (locally) confluent. It is Noetherian if there is no infinite reduction sequence $a_{1} \rightarrow_{\mathcal{T}} a_{2} \rightarrow_{\mathcal{T}} \ldots$ with each $a_{i} \neq a_{i+1}$. If the rewriting rules $U \rightarrow_{\mathcal{T}} V$ always satisfy $U \succ V$ for a well-ordering $\prec$, the rewriting system $\mathcal{T}$ is obviously Noetherian. Even though local confluence does not imply confluence, a Noetherian rewriting system satisfies Newman's Lemma; see also [84]:

Theorem 5.1 (Newman's Lemma [106]) A Noetherian rewriting system is confluent if and only if it is locally confluent.

In order to check a Noetherian rewriting system for confluence it therefore suffices to check it for local confluence only. In Section 5.4. we show that local confluence of our (possibly infinite) rewriting system can be checked algorithmically.

Since $\mathcal{X}^{*}$ is well-ordered, each non-empty set contains a least element. In particular, each equivalence class in $\mathcal{X}^{*} / \sim_{\mathcal{T}}$ contains a (unique) least element. For $W \in \mathcal{X}^{*}$, the least representative of the equivalence class which contains the element $W$ is the canonical 
form of $W$ with respect to $\prec$ and $\sim_{\mathcal{T}}$. The canonical form of an element $W \in \mathcal{X}^{*}$ and irreducibility are related by the following well-known

Lemma 5.2 Let $\mathcal{T}$ be a rewriting system that generates the monoid congruence $\sim_{\mathcal{T}}$. Each canonical form with respect to $\prec$ and $\sim_{\mathcal{T}}$ is irreducible. If $\mathcal{T}$ is confluent, each irreducible element is a canonical form.

Proof. Suppose that $W \in \mathcal{X}^{*}$ is a canonical form that is not irreducible. Then there exist $A, B, P, Q \in \mathcal{X}^{*}$ with $W=A P B$ and a rewriting rule $P \rightarrow \mathcal{T} Q$. The element $V=A Q B$ satisfies $V \sim_{\mathcal{T}} W$ and $W \succ V$; this, however, contradicts our assumption that $W$ is a canonical form and thus a least representative of the equivalence class containing $W$.

Suppose that $W$ is irreducible with respect to the confluent rewriting system $\mathcal{T}$. Let $V$ be the canonical form of $W$ with respect to $\prec$ and $\sim_{\mathcal{T}}$. Then $W \succeq V$ and $W \sim_{\mathcal{T}} V$. By definition of monoid congruence $\sim_{\mathcal{T}}$, there exists a sequence $W=W_{0}, W_{1}, \ldots, W_{n}=V$ so that $W_{i}=A_{i} P_{i} B_{i}, W_{i+1}=A_{i} Q_{i} B_{i}$, and either $P_{i} \rightarrow_{\mathcal{T}} Q_{i}$ or $Q_{i} \rightarrow_{\mathcal{T}} P_{i}$. Since $W=W_{0}$ is irreducible, we have the rewriting rule $Q_{0} \rightarrow_{\mathcal{T}} P_{0} \in \mathcal{T}$. Thus $W_{1} \rightarrow_{\mathcal{T}} W$. For a positive integer $i$, assume that $W_{i} \rightarrow_{\mathcal{T}}^{*} W$ holds. Since $W_{i}=A_{i} P_{i} B_{i}, W_{i+1}=A_{i} Q_{i} B_{i}$, and either $P_{i} \rightarrow_{\mathcal{T}} Q_{i}$ or $Q_{i} \rightarrow_{\mathcal{T}} P_{i}$, we either have $W_{i+1} \rightarrow_{\mathcal{T}} W_{i}$ or $W_{i} \rightarrow_{\mathcal{T}} W_{i+1}$. If $W_{i+1} \rightarrow_{\mathcal{T}} W_{i}$, then $W_{i+1} \rightarrow_{\mathcal{T}}^{*} W$. If, on the other hand, $W_{i} \rightarrow_{\mathcal{T}} W_{i+1}$ holds, then we have both $W_{i} \rightarrow_{\mathcal{T}} W_{i+1}$ and $W_{i} \rightarrow_{\mathcal{T}}^{*} W$. Because the rewriting system $\mathcal{T}$ is confluent, there exists $U \in \mathcal{X}^{*}$ so that $W \rightarrow_{\mathcal{T}}^{*} U$ and $W_{i+1} \rightarrow_{\mathcal{T}}^{*} U$. Since $W$ is irreducible, though, we have $W=U$. Thus $W_{i} \rightarrow_{\mathcal{T}}^{*} W$. By induction on $i$, we obtain that $V=W_{n} \rightarrow_{\mathcal{T}}^{*} W$ which implies that $V \succeq W$. Since $V$ is the least representative of the equivalence class that contains $W$, we have that $W=V$.

Since a rewriting system $\mathcal{T}$ allows us to rewrite each element to an irreducible element that represents the same element of the monoid, we immediately obtain

Corollary 5.3 A confluent rewriting system $\mathcal{T}$ allows one to compute the canonical form with respect to $\prec$ and $\sim_{\mathcal{T}}$ for each element in the monoid.

In the following, we consider the free monoid $\mathcal{S}(\mathcal{X}, \Phi)$ that is generated by the Cartesian product $\mathcal{X} \times \Phi^{*}=\left\{(x, \sigma) \mid x \in \mathcal{X}, \sigma \in \Phi^{*}\right\}$ where $\mathcal{X}$ is a finite alphabet and $\Phi^{*}$ denotes the free monoid which is generated by the finite alphabet $\Phi$.

The monoid $\mathcal{S}(\mathcal{X}, \Phi)$ carries an action of the free monoid $\Phi^{*}$ : For each $\sigma \in \Phi$, the map

$$
\widehat{\sigma}: \mathcal{X} \times \Phi^{*} \rightarrow \mathcal{S}(\mathcal{X}, \Phi),(x, \psi) \mapsto(x, \psi \sigma),
$$

extends to a monoid homomorphism $\widehat{\sigma}: \mathcal{S}(\mathcal{X}, \Phi) \rightarrow \mathcal{S}(\mathcal{X}, \Phi)$. This homomorphism maps $w=\left(x_{1}, \psi_{1}\right) \cdots\left(x_{n}, \psi_{n}\right) \in \mathcal{S}(\mathcal{X}, \Phi)$ to $w^{\widehat{\sigma}}=\left(x_{1}, \psi_{1} \sigma\right) \cdots\left(x_{n}, \psi_{n} \sigma\right)$. The map $\widehat{\sigma}$ is injective but not surjective; as $(x, \varepsilon)$ has no preimage under $\widehat{\sigma}$.

In the following, we consider a rewriting system $\mathcal{T}$ on the monoid $\mathcal{S}(\mathcal{X}, \Phi)$. Even though the rewriting system $\mathcal{T}$ might be infinite, we use the regularities that are induced by the monoid $\Phi^{*}$. For this purpose, we introduce the following notation: 
Definition 5.4 Suppose that $\prec$ is a $\Phi$-invariant reduction ordering (i.e., the ordering $\prec$ is $\Phi$-invariant if $U \prec V$ always implies $U^{\sigma} \prec V^{\sigma}$ for each $\left.\sigma \in \Phi^{*}\right)$. For a rewriting system $\mathcal{T}$ on the free monoid $\mathcal{S}(\mathcal{X}, \Phi)$, a $\Phi$-generating set is a subset $\mathcal{R} \subseteq \mathcal{T}$ so that

$$
\mathcal{T}=\left\{U^{\widehat{\sigma}} \rightarrow V^{\widehat{\sigma}} \mid U \rightarrow V \in \mathcal{R} \text { and } \sigma \in \Phi^{*}\right\} .
$$

A rewriting system $\mathcal{T}$ is $\Phi$-finite, if there exists a finite $\Phi$-generating set $\mathcal{R}$.

In Section 5.2, we show that each invariant finite $L$-presentation gives rise to a $\Phi$-finite rewriting system $\mathcal{T}$. Moreover, in Section 5.4, we develop an algorithm that decides whether or not a $\Phi$-finite rewriting system is locally confluent. This algorithm only performs checks within the finite $\Phi$-generating set $\mathcal{R}$.

\subsection{Monoid $L$-Presentations}

In this section, we introduce the notion of a finite monoid $L$-presentation. Furthermore, we derive a monoid $L$-presentation for each invariantly finitely $L$-presented group. From this monoid $L$-presentation, we then obtain a $\Phi$-finite rewriting system for the $L$-presented group. A monoid presentation of the form

$$
\left.M=\langle\mathcal{X}|\left\{U^{\sigma}=V^{\sigma} \mid(U, V) \in R \text { and } \sigma \in \Phi^{*}\right\}\right\rangle
$$

with a finite alphabet $\mathcal{X}$, a finite set $\mathcal{R} \subseteq \mathcal{X}^{*} \times \mathcal{X}^{*}$ of binary relations, and a finite set $\Phi$ of monoid homomorphisms $\mathcal{X}^{*} \rightarrow \mathcal{X}^{*}$ is a finite ascending monoid L-presentation. This definition generalizes the well-known definition for finitely $L$-presented groups:

Proposition 5.5 Each invariantly finitely L-presented group can be defined by an ascending finite monoid L-presentation.

Proof. Let $\langle\mathcal{X}|\mathcal{Q}| \Phi \mid \mathcal{R}\rangle$ be an invariant finite $L$-presentation for a group $G$. Invariance of the $L$-presentation yields that we can assume that $\mathcal{Q}=\emptyset$ holds; otherwise, we replace $\mathcal{R}$ by $\mathcal{R} \cup \mathcal{Q}$ and $\mathcal{Q}$ by $\emptyset$. In order to construct a monoid presentation for $G$, we consider the formal inverses $\mathcal{X}^{-}=\left\{x^{-1} \mid x \in \mathcal{X}\right\}$ with $\mathcal{X} \cap \mathcal{X}^{-}=\emptyset$. Each substitution $\sigma \in \Phi$ translates in an obvious way to a monoid homomorphism $\widehat{\sigma}$ of the free monoid $\mathcal{X}^{ \pm *}$ over $\mathcal{X}^{ \pm}=\mathcal{X} \cup \mathcal{X}^{-}$. An ascending finite monoid $L$-presentation that defines the group $G$ is given by

$$
\left\langle\mathcal{X}^{ \pm} \mid\left\{U^{\widehat{\psi}}=\left(x^{-1}\right)^{\widehat{\psi}} x^{\widehat{\psi}}=x^{\widehat{\psi}}\left(x^{-1}\right)^{\widehat{\psi}}=\varepsilon \mid U \in \mathcal{R}, x \in \mathcal{X}, \widehat{\psi} \in \Phi^{*}\right\}\right\rangle,
$$

see [129, Proposition 4.7].

Similar to group presentations, ascending monoid $L$-presentations satisfy the following

Lemma 5.6 Each substitution $\sigma \in \Phi^{*}$ of an ascending monoid L-presentation induces a monoid homomorphism; that is, the monoid congruence $\sim$ given by the monoid presentation is $\sigma$-invariant so that $U \sim V$ implies $U^{\sigma} \sim V^{\sigma}$. 
Proof. It suffices to prove that, for each $\psi \in \Phi$, the monoid congruence $\sim$ is $\psi$-invariant. For this purpose, suppose that $U, V \in \mathcal{X}^{*}$ satisfy $U \sim V$. Let $\psi \in \Phi$ be given. Denote by $\mathcal{T}=\left\{\left(U^{\sigma}, V^{\sigma}\right) \mid(U, V) \in \mathcal{R}\right.$ and $\left.\sigma \in \Phi^{*}\right\}$ the relations of the monoid presentation in Proposition 5.5. By definition of the monoid congruence $\sim$, there exists a sequence $U=U_{0}, U_{1}, \ldots, U_{n}=V$ so that $U_{i}=A_{i} P_{i} B_{i}, U_{i+1}=A_{i} Q_{i} B_{i}$ for $A_{i}, B_{i}, P_{i}, Q_{i} \in \mathcal{X}^{*}$ and either $\left(P_{i}, Q_{i}\right) \in \mathcal{T}$ or $\left(Q_{i}, P_{i}\right) \in \mathcal{T}$. If $\left(P_{i}, Q_{i}\right) \in \mathcal{T}$ holds, then $\left(P_{i}^{\sigma}, Q_{i}^{\sigma}\right) \in \mathcal{T}$ for $\sigma \in \Phi^{*}$. Thus, for $0 \leq i \leq n$, we have that $U_{i}^{\psi}=\left(A_{i} P_{i} B_{i}\right)^{\psi}=A_{i}^{\psi} P_{i}^{\psi} B_{i}^{\psi}$ and, similarly, $U_{i+1}=A_{i}^{\psi} Q_{i}^{\psi} B_{i}^{\psi}$ for $A_{i}^{\psi}, B_{i}^{\psi}, P_{i}^{\psi}, Q_{i}^{\psi} \in \mathcal{X}^{*}$ with $\left(P_{i}^{\psi}, Q_{i}^{\psi}\right) \in \mathcal{T}$ or $\left(Q_{i}^{\psi}, P_{i}^{\psi}\right) \in \mathcal{T}$. Thus $U^{\psi} \sim V^{\psi}$

From the finite monoid $L$-presentation in Eq. (5.4), we obtain a monoid $L$-presentation over the Cartesian product $\mathcal{X} \times \Phi^{*}$ :

Theorem 5.7 Let $M$ denote the monoid that is defined by the ascending finite monoid L-presentation $\left\langle\mathcal{X} \mid\left\{U^{\psi}=V^{\psi} \mid(U, V) \in \mathcal{R}, \psi \in \Phi^{*}\right\}\right\rangle$. For each $x \in \mathcal{X}$ and $\sigma \in \Phi$, we write $x^{\sigma}=\xi_{1} \cdots \xi_{\ell}$ with each $\xi_{i} \in \mathcal{X}$. For each relation $(U, V) \in \mathcal{R}$, we write $U=u_{1} \cdots u_{n}$ and $V=v_{1} \cdots v_{m}$ with each $u_{i}, v_{j} \in \mathcal{X}$. The monoid $M$ is isomorphic to the monoid defined by the monoid presentation

$$
N=\left\langle\mathcal{X} \times \Phi^{*} \mid\left\{U^{\widehat{\psi}}=V^{\widehat{\psi}} \mid(U, V) \in \mathcal{V}, \psi \in \Phi^{*}\right\}\right\rangle
$$

where, for each $\varphi \in \Phi^{*}, \widehat{\varphi}$ denotes the monoid endomorphism of the free monoid $\mathcal{S}(\mathcal{X}, \Phi)$ that is induced by the map $\widehat{\varphi}: \mathcal{X} \times \Phi^{*} \rightarrow \mathcal{S}(\mathcal{X}, \Phi),(x, \psi) \mapsto(x, \psi \varphi)$ while the set $\mathcal{V}$ is given by

$$
\begin{aligned}
\mathcal{V} & =\left\{\left(\left(u_{1}, \varepsilon\right) \cdots\left(u_{n}, \varepsilon\right),\left(v_{1}, \varepsilon\right) \cdots\left(v_{m}, \varepsilon\right)\right) \mid\left(u_{1} \cdots u_{n}, v_{1} \cdots v_{m}\right) \in \mathcal{R}\right\} \\
& \cup\left\{\left((x, \sigma),\left(\xi_{1}, \varepsilon\right) \cdots\left(\xi_{\ell}, \varepsilon\right)\right) \mid \sigma \in \Phi, x \in \mathcal{X} \text { with } x^{\sigma}=\xi_{1} \cdots \xi_{\ell}\right\} .
\end{aligned}
$$

Proof. By Lemma 5.6, each substitution $\widehat{\varphi}$ of the free monoid $\mathcal{S}(\mathcal{X}, \Phi)$ induces a monoid endomorphism of $M$. In fact, relations are mapped to relations. Define a monoid homomorphism $\pi: \mathcal{S}(\mathcal{X}, \Phi) \rightarrow \mathcal{X}^{*}$ by the map

$$
\pi:(x, \psi) \mapsto x^{\psi}, \quad \text { for each } x \in \mathcal{X} \text { and } \psi \in \Phi^{*} .
$$

Since $\{(x, \varepsilon) \mid x \in \mathcal{X}\} \subseteq \mathcal{S}(\mathcal{X}, \Phi)$ holds, the image of $\pi$ contains the basis $\mathcal{X}$. Thus, $\pi$ is onto and it induces a surjective homomorphism $\mathcal{S}(\mathcal{X}, \Phi) \rightarrow M$. In order to prove that $\pi$ induces a surjective homomorphism $N \rightarrow M$, it suffices to prove that each relation of $N$ is mapped to a relation in $M$. For $\psi \in \Phi^{*}$ and $\left(u_{1} \cdots u_{n}, v_{1} \cdots v_{m}\right) \in \mathcal{R}$, the relation $\left(\left(u_{1}, \varepsilon\right) \cdots\left(u_{n}, \varepsilon\right)\right)^{\widehat{\psi}}=\left(\left(v_{1}, \varepsilon\right) \cdots\left(v_{m}, \varepsilon\right)\right)^{\widehat{\psi}}$ of the monoid $N$ is mapped as follows:

$$
\begin{aligned}
\left(\left(u_{1}, \varepsilon\right) \cdots\left(u_{n}, \varepsilon\right)\right)^{\widehat{\psi} \pi} & =\left(\left(u_{1}, \psi\right) \cdots\left(u_{n}, \psi\right)\right)^{\pi}=u_{1}^{\psi} \cdots u_{n}^{\psi} \\
\left(\left(v_{1}, \varepsilon\right) \cdots\left(v_{m}, \varepsilon\right)\right)^{\widehat{\psi} \pi} & =\left(\left(v_{1}, \psi\right) \cdots\left(v_{m}, \psi\right)\right)^{\pi}=v_{1}^{\psi} \cdots v_{m}^{\psi}
\end{aligned}
$$


and $u_{1}^{\psi} \cdots u_{n}^{\psi}=v_{1}^{\psi} \cdots v_{m}^{\psi}$ is a relation in $M$. The relation $(x, \sigma)^{\widehat{\psi}}=\left(\left(\xi_{1}, \varepsilon\right) \cdots\left(\xi_{\ell}, \varepsilon\right)\right)^{\widehat{\psi}}$ is mapped to

$$
\begin{aligned}
(x, \sigma)^{\widehat{\psi} \pi} & =(x, \sigma \psi)^{\pi}=x^{\sigma \psi} \\
\left(\left(\xi_{1}, \varepsilon\right) \cdots\left(\xi_{\ell}, \varepsilon\right)\right)^{\widehat{\psi} \pi} & =\left(\left(\xi_{1}, \psi\right) \cdots\left(\xi_{\ell}, \psi\right)\right)^{\pi}=\xi_{1}^{\psi} \cdots \xi_{\ell}^{\psi}
\end{aligned}
$$

and $x^{\sigma \psi}=\xi_{1}^{\psi} \cdots \xi_{\ell}^{\psi}$ is a relation in $M$ since each substitution $\psi \in \Phi^{*}$ induces a homomorphism of $M$ and $x^{\sigma}=\xi_{1} \cdots \xi_{\ell}$ is a relation in $M$. Thus, $\pi$ induces a surjective homomorphism $\pi: N \rightarrow M$.

Define a monoid homomorphism $\gamma: \mathcal{X}^{*} \rightarrow \mathcal{S}(\mathcal{X}, \Phi)$ that is induced by the map

$$
\gamma: x \mapsto(x, \varepsilon), \quad \text { for each } x \in \mathcal{X} .
$$

Then $\gamma$ defines a homomorphism $\mathcal{X}^{*} \rightarrow N$. We first show that the latter homomorphism is surjective by proving $x^{\psi \gamma}=(x, \psi)$ for each $x \in \mathcal{X}$ and $\psi \in \Phi^{*}$. We prove this by induction on $n=\|\psi\|$. If $n=1$, there exists $\sigma \in \Phi$ with $\psi=\sigma$. Since $x^{\sigma}=\xi_{1} \cdots \xi_{\ell} \in \mathcal{X}^{*}$, we obtain $\left(x^{\sigma}\right)^{\gamma}=\left(\xi_{1} \cdots \xi_{\ell}\right)^{\gamma}=\left(\xi_{1}, \varepsilon\right) \cdots\left(\xi_{\ell}, \varepsilon\right)$. There is a relation $(x, \sigma)=\left(\xi_{1}, \varepsilon\right) \cdots\left(\xi_{\ell}, \varepsilon\right)$ in the monoid $N$. This relation yields $\left(x^{\sigma}\right)^{\gamma}=(x, \sigma)$. Suppose that $n>1$ holds. Then $\psi=\sigma \delta$ with $\sigma \in \Phi, \delta \in \Phi^{*}$, and $\|\delta\|=n-1$. Then $\left(\xi_{1} \cdots \xi_{\ell}\right)^{\delta \gamma}=\left(\xi_{1}^{\delta} \cdots \xi_{\ell}^{\delta}\right)^{\gamma}$. By induction on $n$, it follows that $\left(\xi_{1}^{\delta} \cdots \xi_{\ell}^{\delta}\right)^{\gamma}=\left(\xi_{1}, \delta\right) \cdots\left(\xi_{\ell}, \delta\right)=\left(\left(\xi_{1}, \varepsilon\right) \cdots\left(\xi_{\ell}, \varepsilon\right)\right)^{\widehat{\delta}}$. The relation $(x, \sigma)=\left(\xi_{1}, \varepsilon\right) \cdots\left(\xi_{\ell}, \varepsilon\right)$ and its $\Phi$-iterates yield that $x^{\psi}=x^{\sigma \delta}=\left(\xi_{1} \cdots \xi_{\ell}\right)^{\delta}$ is mapped by $\gamma$ to $\left(x^{\psi}\right)^{\gamma}=\left(\left(\xi_{1}, \varepsilon\right) \cdots\left(\xi_{\ell}, \varepsilon\right)\right)^{\widehat{\delta}}=(x, \sigma)^{\widehat{\delta}}=(x, \sigma \delta)=(x, \psi)$. This shows that the map $\gamma: \mathcal{X}^{*} \rightarrow \mathcal{S}(\mathcal{X}, \Phi)$ induces a surjective homomorphism $\mathcal{X}^{*} \rightarrow N$. Obviously, each relation $u_{1}^{\psi} \cdots u_{n}^{\psi}=v_{1}^{\psi} \cdots v_{m}^{\psi}$ of $M$ is mapped to a relation in $N$. Therefore, $\gamma$ induces a homomorphism $M \rightarrow N$. Clearly, $\pi \gamma=\operatorname{id}_{N}$ and $\gamma \pi=\operatorname{id}_{M}$. Thus, the monoids $M$ and $N$ are isomorphic via $\gamma$ and $\pi$.

In the remainder, we write $x^{\psi}$ for the element $(x, \psi) \in \mathcal{X} \times \Phi^{*}$ so that the free monoid $\mathcal{S}(\mathcal{X}, \Phi)$ becomes

$$
\mathcal{S}(\mathcal{X}, \Phi)=\left\{x_{1}^{\sigma_{1}} \cdots x_{n}^{\sigma_{n}} \mid x_{i} \in \mathcal{X}, \sigma_{i} \in \Phi^{*}\right\} .
$$

This notation indicates that the elements $\sigma_{i} \in \Phi^{*}$ correspond to substitutions of an ascending monoid $L$-presentation. We construct a $\Phi$-invariant reduction ordering on $\mathcal{S}(\mathcal{X}, \Phi)$ in Section 5.3 below; that is, a reduction ordering $\prec$ is $\Phi$-invariant if $U \succ V$ implies $U^{\psi} \succ V^{\psi}$ for each $\psi \in \Phi^{*}$. A $\Phi$-invariant reduction ordering $\prec$ gives rise to a $\Phi$-finite rewriting system $\mathcal{T}$ for the monoid $M$ from Theorem 5.7 ;

Lemma 5.8 Suppose that the free monoid $\mathcal{S}(\mathcal{X}, \Phi)$ admits a $\Phi$-invariant reduction ordering $\prec$. Each invariant finite $L$-presentation of a group gives rise to a $\Phi$-finite rewriting system $\mathcal{T}$ with objects $\mathcal{S}(\mathcal{X}, \Phi)$.

Proof. By Proposition 5.5, an invariantly finitely $L$-presented group can be defined by an ascending finite monoid $L$-presentation

$$
\left\langle\mathcal{X} \mid\left\{U^{\psi}=V^{\psi} \mid(U, V) \in \mathcal{R}, \psi \in \Phi^{*}\right\}\right\rangle .
$$


By Theorem 5.7, we obtain an isomorphic monoid presentation with generators $\mathcal{X} \times \Phi^{*}$. This monoid $L$-presentation is ascending and possibly infinite because $\mathcal{X} \times \Phi^{*}$ might be infinite. However, the relations in the monoid presentation in Theorem 5.7 are $\Phi$-finite with $\Phi$-generating set

$$
\{U=V \mid(U, V) \in \mathcal{R}\} \cup\left\{x^{\sigma}=\xi_{1} \cdots \xi_{\ell} \mid x \in \mathcal{X}, \sigma \in \Phi\right\} .
$$

A $\Phi$-invariant reduction ordering $\prec$ allows us to construct a $\Phi$-finite rewriting system for the monoid in Theorem 5.7

\subsection{A Reduction Ordering on $\mathcal{S}(\mathcal{X}, \Phi)$}

In this section, we construct a $\Phi$-invariant reduction ordering on the free monoid $\mathcal{S}(\mathcal{X}, \Phi)$. For this purpose, we recall some basic constructions first: An ordering $x_{1} \prec x_{2} \prec \cdots \prec$ $x_{n} \prec x_{n+1} \prec \ldots$ on the alphabet $\mathcal{X}$ extends to the free monoid $\mathcal{X}^{*}$ over the alphabet $\mathcal{X}$ as follows: Define $a_{1} \cdots a_{n} \prec b_{1} \cdots b_{m}$ if and only if

(i) $n=\left\|a_{1} \cdots a_{n}\right\|<\left\|b_{1} \cdots b_{m}\right\|=m$ or, otherwise,

(ii) if there exists $1 \leq i \leq m=n$ with $a_{j}=b_{j}$, for each $1 \leq j<i$, and $a_{i} \prec b_{i}$.

The ordering $\prec$ is the length-plus-lexicographic ordering on the free monoid $\mathcal{X}^{*}$. We use the left-to-right version here. However, a right-to-left version could be defined analogously and it also applies to the constructions below.

The length-plus-lexicographic ordering is a total and translation-invariant ordering on the free monoid $\mathcal{X}^{*}$ [129]. It is a well-ordering provided that $\prec$ is a well-ordering on the alphabet $\mathcal{X}$ [129]. For instance, if $\mathcal{X}^{*}$ is finitely generated by $\mathcal{X}=\left\{x_{1}, \ldots, x_{n}\right\}$, the length-plus-lexicographic ordering is a total and translation-invariant well-ordering or, for short, a reduction ordering.

Let $\Phi$ be another finite alphabet and denote by $\Phi^{*}$ the free monoid that is generated by $\Phi$. Then an ordering < of the finite set $\Phi$ extends to the length-plus-lexicographic ordering $<$ on the free monoid $\Phi^{*}$. The ordering $<$ further extends to a total well-ordering $\ll$ on the $m$-folded Cartesian product $\Phi^{*} \times \cdots \times \Phi^{*}$ as follows:

Definition 5.9 Let $<$ denote the length-plus-lexicographic ordering on the free monoid $\Phi^{*}$. Define $\left(\sigma_{1}, \ldots, \sigma_{m}\right) \ll\left(\delta_{1}, \ldots, \delta_{m}\right)$ if and only if there exists $1 \leq i \leq m$ so that $\sigma_{j}=\delta_{j}$, for each $1 \leq j<i$, and $\sigma_{i}<\delta_{i}$.

This definition yields the following

Lemma 5.10 The ordering $\ll$ in Definition 5.9 is a total well-ordering on the $m$-folded Cartesian product $\Phi^{*} \times \cdots \times \Phi^{*}$.

Proof. Let $\left(\sigma_{1}, \ldots, \sigma_{m}\right),\left(\delta_{1}, \ldots, \delta_{m}\right) \in \Phi^{*} \times \cdots \times \Phi^{*}$ be given. Since $<$ is a total ordering on $\Phi^{*}$, for each $1 \leq j \leq m$, we either have $\sigma_{j}<\delta_{j}, \delta_{j}<\sigma_{j}$, or $\sigma_{j}=\delta_{j}$. For the $m$-folded Cartesian product $\Phi^{*} \times \cdots \times \Phi^{*}$ we either have $\left(\sigma_{1}, \ldots, \sigma_{m}\right)=\left(\delta_{1}, \ldots, \delta_{m}\right)$ or there exists 
$1 \leq i<m$ so that $\sigma_{j}=\delta_{j}$, for each $1 \leq j<i$, and either $\sigma_{i}<\delta_{i}$ or $\sigma_{i}>\delta_{i}$. Hence, $\ll$ is a total ordering on $\Phi^{*} \times \cdots \times \Phi^{*}$ as soon as $<$ is a total ordering on $\Phi^{*}$.

Consider the $m$-folded Cartesian product $\Phi^{*} \times \cdots \times \Phi^{*}$. If $m=1$, the ordering $\ll$ in Definition 5.9 coincides with the well-ordering $<$ on $\Phi^{*}$. Obviously, the ordering $\ll$ is a well-ordering in this case. Suppose that $m>1$ holds. Let $\left(\sigma_{1}^{(1)}, \ldots, \sigma_{m}^{(1)}\right) \gg$ $\left(\sigma_{1}^{(2)}, \ldots, \sigma_{m}^{(2)}\right) \gg \ldots$ be descending series in $\Phi^{*} \times \cdots \times \Phi^{*}$. Then $\left(\sigma_{1}^{(1)}, \ldots, \sigma_{m-1}^{(1)}\right) \gg$ $\left(\sigma_{1}^{(2)}, \ldots, \sigma_{m-1}^{(2)}\right) \gg \ldots$ is a descending series in the $(m-1)$-folded Cartesian product. By induction on $m$, there exists $\ell \in \mathbb{N}_{0}$ so that $\left(\sigma_{1}^{(j)}, \ldots, \sigma_{m-1}^{(j)}\right)=\left(\sigma_{1}^{(j+1)}, \ldots, \sigma_{m-1}^{(j+1)}\right)$ for each $j \geq \ell$. For $j \geq \ell$, this yields that $\sigma_{m}^{(j)} \geq \sigma_{m}^{(j+1)} \geq \ldots$ is a descending sequence in the well-ordered monoid $\Phi^{*}$. Thus, there exists $k \geq \ell$ so that $\sigma_{m}^{(j)}=\sigma_{m}^{(j+1)}$, for each $j \geq k$, and hence, $\ll$ is a well-ordering.

The following definition gives a reduction ordering on the free monoid

$$
\mathcal{S}(\mathcal{X}, \Phi)=\left\{x_{1}^{\sigma_{1}} \cdots x_{n}^{\sigma_{n}} \mid x_{i} \in \mathcal{X}, \sigma_{i} \in \Phi^{*}, 1 \leq i \leq n\right\} .
$$

This ordering will be central to our construction of a $\Phi$-finite rewriting system below.

Definition 5.11 Denote the length-plus-lexicographic ordering on $\mathcal{X}^{*}$ by $<$ and let $\ll$ denote the ordering on $\Phi^{*} \times \cdots \times \Phi^{*}$ from Definition 5.9. Then an ordering $\prec$ on the free monoid $\mathcal{S}(\mathcal{X}, \Phi)$ is given as follows: For $x_{1}^{\sigma_{1}} \cdots x_{n}^{\sigma_{n}}, y_{1}^{\delta_{1}} \cdots y_{m}^{\delta_{m}} \in \mathcal{S}(\mathcal{X}, \Phi)$, we define $x_{1}^{\sigma_{1}} \cdots x_{n}^{\sigma_{n}} \prec y_{1}^{\delta_{1}} \cdots y_{m}^{\delta_{m}}$ if and only if

(i) either $x_{1} \cdots x_{n}<y_{1} \cdots y_{m}$ holds or, otherwise,

(ii) if $\left(\sigma_{1}, \ldots, \sigma_{n}\right) \ll\left(\delta_{1}, \ldots, \delta_{n}\right)$.

This definition yields the following

Proposition 5.12 The ordering $\ll$ in Definition 5.11 is a reduction ordering on the free monoid $\mathcal{S}(\mathcal{X}, \Phi)$.

Proof. The ordering in Definition [5.11 is total as both, the length-plus-lexicographic ordering $<$ and the ordering $\ll$ from Definition 5.9 are total orderings.

Suppose that $U_{1} \gg U_{2} \gg \ldots \gg U_{i} \gg U_{i+1} \gg \ldots$ is descending series in $\mathcal{S}(\mathcal{X}, \Phi)$. Since $<$ is a well-ordering on $\mathcal{X}^{*}$, there exists $\ell \in \mathbb{N}_{0}$ so that $U_{i}=x_{1}^{\sigma_{1}^{(i)}} \cdots x_{n}^{\sigma_{n}^{(i)}}$ and $U_{i+1}=x_{1}^{\sigma_{1}^{(i+1)}} \cdots x_{n}^{\sigma_{n}^{(i+1)}}$ for each $i \geq \ell$. It therefore suffices to consider the descending series $\left(\sigma_{1}^{(\ell)}, \ldots, \sigma_{n}^{(\ell)}\right) \gg\left(\sigma_{1}^{(\ell+1)}, \ldots, \sigma_{n}^{(\ell+1)}\right) \gg \ldots$ within the $n$-folded Cartesian product $\Phi^{*} \times \cdots \times \Phi^{*}$. Since $\ll$ is a well-ordering, though, the latter sequence terminates and thus, there exists $k \geq \ell$ so that $\left(\sigma_{1}^{(m)}, \ldots, \sigma_{n}^{(m)}\right)=\left(\sigma_{1}^{(m+1)}, \ldots, \sigma_{n}^{(m+1)}\right)$ for each $m \geq k$. In particular, for each $i \geq k$, we have $U_{i}=x_{1}^{\sigma_{1}^{(i)}} \cdots x_{n}^{\sigma_{n}^{(i)}}=x_{1}^{\sigma_{1}^{(i+1)}} \cdots x_{n}^{\sigma_{n}^{(i+1)}}=U_{i+1}$ and hence, the ordering $\prec$ is a well-ordering.

For proving translation invariance of the ordering $\prec$, it suffices to prove that, for each $x \in \mathcal{X}$ and $\sigma \in \Phi^{*}$, we both have $x^{\sigma} U \prec x^{\sigma} V$ and $U x^{\sigma} \prec V x^{\sigma}$ whenever $U \prec V$ 
holds. However, this follows immediately from the construction of the ordering $\prec$ in Definition 5.11 and the fact that the length-plus-lexicographic ordering on $\mathcal{X}^{*}$ is translation invariant; see [129, Proposition 1.5].

The ordering $\prec$ in Definition 5.11 is $\Phi$-invariant:

Lemma 5.13 For $U, V \in \mathcal{S}(\mathcal{X}, \Phi)$ and $\psi \in \Phi^{*}, U \prec V$ implies $U^{\psi} \prec V^{\psi}$.

Proof. Write $U=x_{1}^{\sigma_{1}} \cdots x_{n}^{\sigma_{n}}$ and $V=y_{1}^{\delta_{1}} \cdots y_{m}^{\delta_{m}}$ with each $x_{i}, y_{j} \in \mathcal{X}$ and $\sigma_{i}, \delta_{j} \in \Phi^{*}$. For $\psi \in \Phi$, we have

$$
U^{\psi}=x_{1}^{\sigma_{1} \psi} \cdots x_{n}^{\sigma_{n} \psi} \quad \text { and } \quad V^{\psi}=y_{1}^{\delta_{1} \psi} \cdots y_{m}^{\delta_{m} \psi} .
$$

Since $U \prec V$ holds, we either have $x_{1} \cdots x_{n}<y_{1} \cdots y_{m}$ in the length-plus-lexicographic ordering $<$ on $\mathcal{X}^{*}$ or $\left(\sigma_{1}, \ldots, \sigma_{n}\right) \ll\left(\delta_{1}, \ldots, \delta_{n}\right)$ in the ordering from Definition [5.9, In the first case, the images $U^{\psi}$ and $V^{\psi}$ satisfy $x_{1} \cdots x_{n}<y_{1} \cdots y_{m}$. Thus $U^{\psi} \prec V^{\psi}$. In the second case, we would have $x_{1} \cdots x_{n}=y_{1} \cdots y_{m}$ and $\left(\sigma_{1}, \ldots, \sigma_{n}\right) \ll\left(\delta_{1}, \ldots, \delta_{n}\right)$. Since the length-plus-lexicographic ordering on $\Phi^{*}$ is translation invariant, the latter implies $\left(\sigma_{1} \psi, \ldots, \sigma_{n} \psi\right) \ll\left(\delta_{1} \psi, \ldots, \delta_{n} \psi\right)$. Thus $U^{\psi} \prec V^{\psi}$.

We finally note the following

Remark 5.14 Similar to the Knuth-Bendix completion for finitely presented groups, the reduction ordering is flexible; for instance, we need to specify the orderings on the generating sets $\mathcal{X}$ and $\Phi$ of the free monoids $\mathcal{X}^{*}$ and $\Phi^{*}$. Furthermore, we can also use the right-to-left version of the length-plus-lexicographic ordering. This provides a selection of reduction orderings that can be applied in the Knuth-Bendix completion algorithm.

\subsection{The Critical Pair Lemma}

In the following, we establish a Critical Pair Lemma for a $\Phi$-finite rewriting system. This lemma enables us to decide whether or not a $\Phi$-finite rewriting system is locally confluent. The Critical Pair Lemma below generalizes [129, Proposition 3.1] for finite presentations.

Suppose that $\mathcal{T}$ is a $\Phi$-finite rewriting system with $\Phi$-generating set $\mathcal{R}$. Then an element $W \in \mathcal{S}(\mathcal{X}, \Phi)$ reduces with respect to $\mathcal{T}$ if there exist $A, B, P, Q \in \mathcal{S}(\mathcal{X}, \Phi)$ and $\sigma \in \Phi^{*}$ so that $W=A P^{\sigma} B$ and $P \rightarrow Q$ is a rewriting rule in the $\Phi$-generating set $\mathcal{R}$. In order to find the minimal words $W \in \mathcal{S}(\mathcal{X}, \Phi)$ on which local confluence fails we therefore need to recognize the left-side $P$ of the rewriting rule $P \rightarrow Q$ as a 'subword' of $W=A P^{\sigma} B$ even if $\sigma$ is non-trivial. More precisely, we use the following definition of a $\Phi$-subword:

Definition 5.15 For an element $W=x_{1}^{\sigma_{1}} \cdots x_{n}^{\sigma_{n}} \in \mathcal{S}(\mathcal{X}, \Phi)$, a $\Phi$-subword has the form $x_{i}^{\delta_{i}} \cdots x_{j}^{\delta_{j}}$ with $1 \leq i \leq j \leq n$ and there exists $\omega \in \Phi^{*}$ so that $\sigma_{\ell}=\delta_{\ell} \omega$ for each $i \leq \ell \leq j$. An element $V \in \mathcal{S}(\mathcal{X}, \Phi)$ is a proper $\Phi$-subword of $W$ if it is a $\Phi$-subword of $W$ with $W \neq V$. 
The notion of a $\Phi$-subword is more general than the notion of a subword within the free monoid $\mathcal{S}(\mathcal{X}, \Phi)$ as it also incorporates the action of the monoid $\Phi^{*}$. In particular, a $\Phi$-subword $V$ of $W$ is an $\omega$-preimage of subword in the free monoid $\mathcal{S}(\mathcal{X}, \Phi)$. Note that $\sigma_{\ell}=\delta_{\ell} \omega$, for a common $\omega \in \Phi^{*}$, in Definition 5.15 implies that $\delta_{\ell}$ is a prefix of $\sigma_{\ell}$. Moreover, we have the following observation:

Lemma 5.16 Let $\prec$ denote the reduction ordering from Section 5.3. Every $W \in \mathcal{S}(\mathcal{X}, \Phi)$ has only finitely many $\Phi$-subwords. Each proper $\Phi$-subword $U$ of $W$ satisfies $U \prec W$.

Proof. The proof follows immediately from the definitions above.

An element $W \in \mathcal{S}(\mathcal{X}, \Phi)$ is locally confluent if for each $A, B \in \mathcal{S}(\mathcal{X}, \Phi)$ with $W \rightarrow A$ and $W \rightarrow B$, there exists $D \in \mathcal{S}(\mathcal{X}, \Phi)$ so that $A \rightarrow^{*} D$ and $B \rightarrow^{*} D$. A minimal condition for an element $W \in \mathcal{S}(\mathcal{X}, \Phi)$ not to be locally confluent is given by the following proposition (in the style of [129]):

Proposition 5.17 (The Critical Pair Lemma) Let $\mathcal{T}$ be a rewriting system on $\mathcal{S}(\mathcal{X}, \Phi)$ with (possibly infinite) $\Phi$-generating set $\mathcal{R} \subseteq \mathcal{T}$. Suppose that the element $W \in \mathcal{S}(\mathcal{X}, \Phi)$ is not locally confluent but each of its proper $\Phi$-subwords is locally confluent. Then one of the following conditions holds: There exist rewriting rules $P_{1} \rightarrow Q_{1}$ and $P_{2} \rightarrow Q_{2}$ in the $\Phi$-generating set $\mathcal{R}$ so that

(i) $W=P_{1}$ and $W=A P_{2}^{\sigma} B$ for $\sigma \in \Phi^{*}$ and $A, B \in \mathcal{S}(\mathcal{X}, \Phi)$.

(ii) $W=P_{1}^{\sigma}$ and $W=A^{\sigma} P_{2} B^{\sigma}$ for $\sigma \in \Phi^{*}$ and non-empty words $A, B \in \mathcal{S}(\mathcal{X}, \Phi)$.

(iii) $W$ can be written $(A B)^{\sigma} C$ or $A(B C)^{\sigma}$ for non-empty words $A, B, C \in \mathcal{S}(\mathcal{X}, \Phi)$, $\sigma \in \Phi^{*}$, and either

- $P_{1}=A B$ and $P_{2}=B^{\sigma} C$, or

- $P_{1}=A B^{\sigma}$ and $P_{2}=B C$.

Proof. We generalize the proof of [129, Proposition 3.1]. If $W \in \mathcal{S}(\mathcal{X}, \Phi)$ is not locally confluent, there exist $A_{1}, P_{1}, Q_{1}, B_{1}, A_{2}, P_{2}, Q_{2}, B_{2} \in \mathcal{S}(\mathcal{X}, \Phi)$ and $\sigma, \delta \in \Phi^{*}$ so that

(i) $W=A_{1} P_{1}^{\sigma} B_{1}$ and $W=A_{2} P_{2}^{\delta} B_{2}$,

(ii) there are rewriting rules $P_{1} \rightarrow Q_{1}$ and $P_{2} \rightarrow Q_{2}$ in $\mathcal{R}$,

(iii) there does not exist $V \in \mathcal{S}(\mathcal{X}, \Phi)$ with $A_{1} Q_{1}^{\sigma} B_{1} \rightarrow^{*} V$ and $A_{2} Q_{2}^{\delta} B_{2} \rightarrow^{*} V$.

First, assume that $P_{1}$ and $P_{2}$ do not overlap; i.e., we have $W=A_{1} P_{1}^{\sigma} C P_{2}^{\delta} B_{2}$ for some $C \in \mathcal{S}(\mathcal{X}, \Phi)$. Using the rewriting rule $P_{1} \rightarrow Q_{1}$, we obtain $U_{1}=A_{1} Q_{1}^{\sigma} C P_{2}^{\delta} B_{2}$ while the rule $P_{2} \rightarrow Q_{2}$ gives us $U_{2}=A_{1} P_{1}^{\sigma} C Q_{2}^{\delta} B_{2}$. Both $U_{1}$ and $U_{2}$ reduce to the extended word $V=A_{1} Q_{1}^{\sigma} C Q_{2}^{\delta} B_{2}$; this contradicts the third condition on $W$. Therefore, $P_{1}$ and $P_{2}$ do overlap. This yields either one of the following conditions holds:

- There exist positive integers $1 \leq i \leq j \leq n$ with $P_{1}^{\sigma}=x_{1}^{\sigma_{1} \sigma} \cdots x_{i}^{\sigma_{i} \sigma} \cdots x_{j}^{\sigma_{j} \sigma} \cdots x_{n}^{\sigma_{n} \sigma}$ and $P_{2}^{\delta}=x_{i}^{\sigma_{i} \sigma} \cdots x_{j}^{\sigma_{j} \sigma}$. Write $P_{1}^{\sigma}=A^{\sigma} P_{2}^{\delta} C^{\sigma}$ in this case. 
- There exist positive integers $1<i \leq j<m$ with $P_{1}^{\sigma}=x_{1}^{\sigma_{1} \sigma} \cdots x_{i}^{\sigma_{i} \sigma} \cdots x_{j}^{\sigma_{j} \sigma}$, $P_{2}^{\delta}=x_{i}^{\delta_{i} \delta} \cdots x_{j}^{\delta_{j} \delta} \cdots x_{m}^{\delta_{m} \delta}$ and $\delta_{\ell} \delta=\sigma_{\ell} \sigma$ for $i \leq \ell \leq j$. Write $P_{1}^{\sigma}=A^{\sigma} B^{\sigma}$ and $P_{2}^{\delta}=B^{\delta} C^{\delta}$ for non-empty words $A, B, C \in \mathcal{S}(\mathcal{X}, \Phi)$ with $B^{\sigma}=B^{\delta}$.

Consider the first case above. Then $W=A_{1} P_{1}^{\sigma} B_{1}=A_{1} A^{\sigma} P_{2}^{\delta} C^{\sigma} B_{1}, A_{2}=A_{1} A^{\sigma}$, and $B_{2}=C^{\sigma} B_{1}$. Suppose that $A_{1} \neq \varepsilon$ or $B_{1} \neq \varepsilon$ holds. Then $P_{1}^{\sigma}=A^{\sigma} P_{2}^{\delta} C^{\sigma}$ is a proper $\Phi$ subword of $W=A_{1} A^{\sigma} P_{2}^{\delta} C^{\sigma} B_{1}$. Since each proper $\Phi$-subword of $W$ is locally confluent, there exists $V \in \mathcal{S}(\mathcal{X}, \Phi)$ so that $P_{1}^{\sigma} \rightarrow Q_{1}^{\sigma} \rightarrow^{*} V$ and $A^{\sigma} P_{2}^{\delta} C^{\sigma} \rightarrow A^{\sigma} Q_{2}^{\delta} C^{\sigma} \rightarrow^{*} V$. This yields that

$$
A_{1} P_{1}^{\sigma} B_{1} \rightarrow A_{1} Q_{1}^{\sigma} B_{1} \rightarrow^{*} A_{1} V B_{1}
$$

and

$$
A_{2} P_{2}^{\delta} B_{2} \rightarrow A_{2} Q_{2}^{\delta} B_{2}=A_{1} A^{\sigma} Q_{2}^{\delta} C^{\sigma} B_{1} \rightarrow^{*} A_{1} V B_{1} ;
$$

this contradicts the third condition on $W$. Thus $A_{1}=\varepsilon=B_{1}$ and $W=A^{\sigma} P_{2}^{\delta} C^{\sigma}=P_{1}^{\sigma}$.

Suppose that $\omega \in \Phi^{*}$ is a common suffix of $\sigma$ and $\delta$ so that $\delta=\bar{\delta} \omega$ and $\sigma=\bar{\sigma} \omega$ hold for $\bar{\delta}, \bar{\sigma} \in \Phi^{*}$. Consider $U=A^{\bar{\sigma}} P_{2}^{\bar{\delta}} C^{\bar{\sigma}}=P_{1}^{\bar{\sigma}} \in \mathcal{S}(\mathcal{X}, \Phi)$. If the suffix $\omega \in \Phi^{*}$ is non-trivial, $U$ is a proper $\Phi$-subword of $W=A^{\sigma} P_{2}^{\delta} C^{\sigma}$. Since each proper $\Phi$-subword of $W$ is locally confluent, there exists $V \in \mathcal{S}(\mathcal{X}, \Phi)$ so that $U=P_{1}^{\bar{\sigma}} \rightarrow Q_{1}^{\bar{\sigma}} \rightarrow^{*} V$ and $U=A^{\bar{\sigma}} P_{2}^{\bar{\delta}} C^{\bar{\sigma}} \rightarrow A^{\bar{\sigma}} Q_{2}^{\bar{\delta}} C^{\bar{\sigma}} \rightarrow^{*} V$. Hence $Q_{1}^{\sigma} \rightarrow^{*} V^{\omega}, A^{\sigma} Q_{2}^{\delta} C^{\sigma} \rightarrow^{*} V^{\omega}$ as well as

$$
A_{1} Q_{1}^{\sigma} B_{1}=Q_{1}^{\sigma} \rightarrow^{*} V^{\omega}
$$

and

$$
A_{2} Q_{2}^{\delta} B_{2}=A_{1} A^{\sigma} Q_{2}^{\delta} C^{\sigma} B_{1}=A^{\sigma} Q_{2}^{\delta} C^{\sigma} \rightarrow^{*} V^{\omega} ;
$$

this contradicts the third assumption on $W$. Therefore, the common suffix $\omega$ is trivial. As $P_{1}$ and $P_{2}$ have the form $P_{1}^{\sigma}=x_{1}^{\sigma_{1} \sigma} \cdots x_{i}^{\sigma_{i} \sigma} \cdots x_{j}^{\sigma_{j} \sigma} \cdots x_{n}^{\sigma_{n} \sigma}$ and $P_{2}^{\delta}=x_{i}^{\sigma_{i} \sigma} \cdots x_{j}^{\sigma_{j} \sigma}$, $\delta$ is a common suffix of $\sigma_{\ell} \sigma$, for each $i \leq \ell \leq j$. We obtain either one of the following conditions:

- If $\sigma=\delta$ holds, both $\sigma$ and $\delta$ are trivial. Thus $W=A P_{2} C=P_{1}$ for $A, C \in \mathcal{S}(\mathcal{X}, \Phi)$.

- If $\|\sigma\|<\|\delta\|$ holds, $\sigma$ is a suffix of $\delta$ and hence $\sigma$ is trivial. Thus $W=A P_{2}^{\psi} C=P_{1}$ for $A, C \in \mathcal{S}(\mathcal{X}, \Phi)$ and $\psi \in \Phi^{*}$.

- If $\|\sigma\|>\|\delta\|$ holds, $\delta$ is a suffix of $\sigma$ and hence $\delta$ is trivial. Thus $W=A^{\psi} P_{2} C^{\psi}=P_{1}^{\psi}$ for $A, C \in \mathcal{S}(\mathcal{X}, \Phi)$ and $\psi \in \Phi^{*}$.

The first two conditions correspond to (i) in Proposition 5.17 while the last condition correspond to either (i), if both $A$ and $B$ are empty, or it corresponds to (ii) of Proposition 5.17 otherwise.

Consider the second case where we have that $P_{1}^{\sigma}=A^{\sigma} B^{\sigma}$ and $P_{2}^{\delta}=B^{\delta} C^{\delta}$ for nonempty words $A, B, C \in \mathcal{S}(\mathcal{X}, \Phi)$ with $B^{\sigma}=B^{\delta}$. Then $W=A_{1} P_{1}^{\sigma} B_{1}=A_{1} A^{\sigma} B^{\sigma} B_{1}$ and $W=A_{2} P_{2}^{\delta} B_{2}=A_{2} B^{\delta} C^{\delta} B_{2}$. Moreover, we have

$$
W=A_{1} A^{\sigma} B^{\sigma} C^{\delta} B_{2}=A_{1} A^{\sigma} B^{\delta} C^{\delta} B_{2},
$$


as well as $B_{1}=C^{\delta} B_{2}$ and $A_{2}=A_{1} A^{\sigma}$. Suppose that $A_{1} \neq \varepsilon$ or $B_{2} \neq \varepsilon$ holds. Then $U=A^{\sigma} B^{\sigma} C^{\delta}=A^{\sigma} B^{\delta} C^{\delta}$ is a proper $\Phi$-subword of $W$. Since each proper $\Phi$-subword of $W$ is locally confluent, there exists $V \in \mathcal{S}(\mathcal{X}, \Phi)$ so that

$$
U=A^{\sigma} B^{\sigma} C^{\delta} \rightarrow Q_{1}^{\sigma} C^{\delta} \rightarrow^{*} V \text { and } U=A^{\sigma} B^{\delta} C^{\delta} \rightarrow A^{\sigma} Q_{2}^{\delta} \rightarrow^{*} V .
$$

This yields that

$$
A_{1} P_{1}^{\sigma} B_{1} \rightarrow A_{1} Q_{1}^{\sigma} B_{1}=A_{1} Q_{1}^{\sigma} C^{\delta} B_{2} \rightarrow^{*} A_{1} V B_{2}
$$

and

$$
A_{2} P_{2}^{\delta} B_{2} \rightarrow A_{2} Q_{2}^{\delta} B_{2}=A_{1} A^{\sigma} Q_{2}^{\delta} B_{2} \rightarrow^{*} A_{1} V B_{2}
$$

this contradicts the third condition on $W$. Thus $A_{1}=\varepsilon=B_{2}$ and $W=A^{\sigma} B^{\sigma} C^{\delta}=$ $A^{\sigma} B^{\delta} C^{\delta}$ with $P_{1}^{\sigma}=A^{\sigma} B^{\sigma}, P_{2}^{\delta}=B^{\delta} C^{\delta}$, and $B^{\sigma}=B^{\delta}$.

Suppose that $\omega \in \Phi^{*}$ is a common suffix of $\delta$ and $\sigma$ so that $\delta=\bar{\delta} \omega$ and $\sigma=\bar{\sigma} \omega$ hold for $\bar{\sigma}, \bar{\delta} \in \Phi^{*}$. Then $B^{\bar{\delta}}=B^{\bar{\sigma}}$. Consider $U=A^{\bar{\sigma}} B^{\bar{\sigma}} C^{\bar{\delta}}=A^{\bar{\sigma}} B^{\bar{\delta}} C^{\bar{\delta}}$. If the suffix $\omega$ is non-trivial, $U$ is a proper $\Phi$-subword of $W$. Since each proper $\Phi$-subword of $W$ is locally confluent, there exists $V \in \mathcal{S}(\mathcal{X}, \Phi)$ so that

$$
U=A^{\bar{\sigma}} B^{\bar{\sigma}} C^{\bar{\delta}} \rightarrow Q_{1}^{\bar{\sigma}} C^{\bar{\delta}} \rightarrow^{*} V \quad \text { and } \quad U=A^{\bar{\sigma}} B^{\bar{\delta}} C^{\bar{\delta}} \rightarrow A^{\bar{\sigma}} Q_{2}^{\bar{\delta}} \rightarrow^{*} V .
$$

This yields that $Q_{1}^{\sigma} C^{\delta} \rightarrow^{*} V^{\omega}$ and $A^{\sigma} Q_{2}^{\delta} \rightarrow^{*} V^{\omega}$ as well as

$$
A_{1} P_{1}^{\sigma} B_{1} \rightarrow A_{1} Q_{1}^{\sigma} B_{1}=A_{1} Q_{1}^{\sigma} C^{\delta} B_{2} \rightarrow^{*} A_{1} V^{\omega} B_{2}
$$

and

$$
A_{2} P_{2}^{\delta} B_{2} \rightarrow A_{2} Q_{2}^{\delta} B_{2}=A_{1} A^{\sigma} Q_{2}^{\delta} B_{2} \rightarrow^{*} A_{1} V^{\omega} B_{2} ;
$$

this contradicts the third condition on $W$. Thus the common suffix $\omega$ is trivial. Since $B^{\delta}=B^{\sigma}$, we either have that $\delta$ is a suffix of $\sigma$ or vice versa. In particular, this shows that either $\delta$ or $\sigma$ is trivial. We obtain the third condition of Proposition 5.17 .

There are only finitely many checks in Proposition 5.17 to be performed on a $\Phi$-finite rewriting system with a finite $\Phi$-generating set $\mathcal{R}$. Note that condition (i) in Proposition 5.17 yield that there exists a rewriting rule whose left-side is reducible. Similar to [129, rewriting systems where the left-side of a rule is reducible can be improved. A rewriting system $\mathcal{T}$ in which condition (i) of Proposition 5.17 does not occur is called a reduced rewriting system.

If local confluence fails at one of the conditions in Proposition 5.17, we obtain two irreducible elements $U, V \in \mathcal{S}(\mathcal{X}, \Phi)$ which satisfy $W \rightarrow_{\mathcal{T}} U, W \rightarrow_{\mathcal{T}} V$, and $U \neq V$. In this case, the tuple $(U, V) \in \mathcal{S}(\mathcal{X}, \Phi) \times \mathcal{S}(\mathcal{X}, \Phi)$ is called a $\Phi$-overlap of the rewriting rules $P_{1} \rightarrow Q_{1}$ and $P_{2} \rightarrow Q_{2}$. Again, these overlaps generalize the notion in 129 for finitely presented groups. For our algorithm, we need the following

Lemma 5.18 There are only finitely many $\Phi$-overlaps in a $\Phi$-finite rewriting system.

Proof. Since each element $W \in \mathcal{S}(\mathcal{X}, \Phi)$ has only finitely many $\Phi$-subwords and each overlap in Proposition 5.17 contains the left-side of a rewriting rule from a finite $\Phi$ generating set $\mathcal{R}$, there are only finitely many overlaps of rewriting rules. 


\subsection{A Generalized Knuth-Bendix Completion}

So far, we have introduced the basics for a Knuth-Bendix completion as outlined in [129, Section 2]; see also [86]. The overall idea of a Knuth-Bendix completion is quite simple: Since, by Newman's Lemma 5.1 , local confluence of a Noetherian rewriting system already implies confluence, it suffices to check a $\Phi$-finite rewriting system for local confluence only. For this purpose, the Critical Pair Lemma 5.17 applies. If we apply Lemma 5.17 to a finite $\Phi$-generating set, we either prove local confluence of the rewriting system or we would have found an overlap $(U, V) \in \mathcal{S}(\mathcal{X}, \Phi) \times \mathcal{S}(\mathcal{X}, \Phi)$. In the latter case, the $\Phi$ overlap satisfies $U \sim V$ and either $U \succ V$ or $U \prec V$. Suppose that $U \succ V$ holds. Adding the rewriting rule $U \rightarrow V$ to the $\Phi$-generating set $\mathcal{R}$ of the rewriting system $\mathcal{T}$ does not change the monoid congruence $\sim \mathcal{T}$. We can continue the process until we prove local confluence of the obtained rewriting system. Of course termination of this approach is central here. In the following, we describe a first-hand approach to a KnuthBendix completion for invariantly finitely $L$-presented groups. Moreover, we prove that it terminates if the monoid $L$-presentation with generators $\mathcal{X} \times \Phi^{*}$ admits a $\Phi$-finite confluent rewriting system with respect to $\prec$.

Let $G$ be an invariantly finitely $L$-presented group. Then, by Proposition 5.5 and Theorem 5.7, $G$ admits an ascending monoid $L$-presentation of the form

$$
\left.\left\langle\mathcal{X} \times \Phi^{*}\right|\left\{U^{\psi}=V^{\psi} \mid(U, V) \in \mathcal{S} \text { and } \psi \in \Phi^{*}\right\}\right\rangle .
$$

The monoid congruence $\sim$ is generated by the $\Phi$-finite rewriting system

$$
\mathcal{T}=\left\{P^{\psi} \rightarrow Q^{\psi} \mid P \succ Q,(P, Q) \in \mathcal{S} \text { or }(Q, P) \in \mathcal{S}, \psi \in \Phi^{*}\right\}
$$

with $\Phi$-generating set $\mathcal{R}=\{P \rightarrow Q \mid P \succ Q,(P, Q) \in \mathcal{S}$ or $(Q, P) \in \mathcal{S}\}$. The algorithm LKnuthBendix in Algorithm 5.1 below takes as input the finite $\Phi$-generating set $\mathcal{R}$ and it attempts to compute a finite $\Phi$-generating set for a confluent $\Phi$-finite rewriting system $\mathcal{T}$ that also defines the monoid congruence $\sim \mathcal{T}$. The algorithm LKNUThBEndiX terminates whenever a confluent $\Phi$-finite rewriting system exists:

Theorem 5.19 Let $\prec$ be a reduction ordering on $\mathcal{S}(\mathcal{X}, \Phi)$. If the monoid defined by the monoid presentation

$$
\left\langle\mathcal{X} \times \Phi^{*}\right|\left\{U^{\psi}=V^{\psi}\left|(U, V) \in \mathcal{R}, \psi \in \Phi^{*}\right\rangle\right.
$$

admits a confluent $\Phi$-finite rewriting system $\mathcal{T}$ with respect to $\prec$, the algorithm LKNUTHBENDIX terminates and it computes a finite $\Phi$-generating set $\mathcal{R}$ for $\mathcal{T}$.

Proof. We generalize the proof from [129, Proposition 5.1]. Suppose that the algorithm LKnuthBendix in Algorithm 5.1 does not terminate. Then it constructs an infinite sequence of rewriting rules $P_{i} \rightarrow Q_{i}$ where both $P_{i}$ and $Q_{i}$ are irreducible with respect to the $\Phi$-finite rewriting system $\mathcal{Z}_{i}=\left\{P_{j}^{\psi} \rightarrow Q_{j}^{\psi} \mid 1 \leq j<i, \psi \in \Phi^{*}\right\}$. Denote by $\mathcal{Y}_{i}=\left\{P_{j} \rightarrow Q_{j} \mid 1 \leq j<i\right\}$ a $\Phi$-generating set for $\mathcal{Z}_{i}$. Write $\mathcal{Z}=\bigcup_{i \in \mathbb{N}_{0}} \mathcal{Z}_{i}$ and 


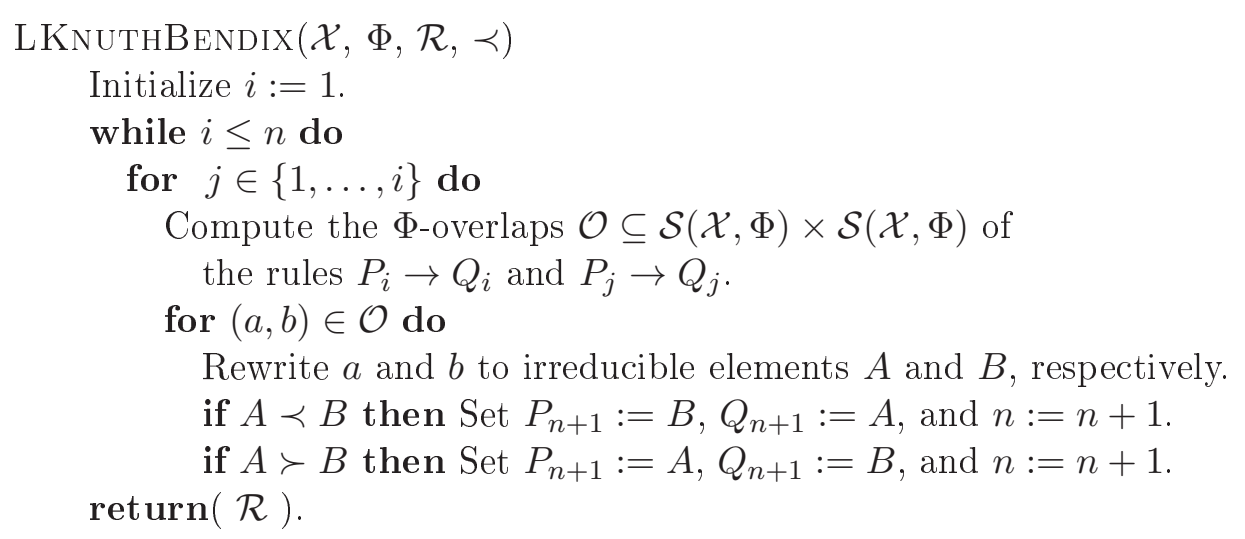

Algorithm 5.1: A Knuth-Bendix completion algorithm

$\mathcal{Y}=\bigcup_{i \in \mathbb{N}_{0}} \mathcal{Y}_{i}$. We first prove that the rewriting system $\mathcal{Z}$ is confluent. Since $\mathcal{Z}$ is Noetherian, it suffices to prove that it is local confluent.

Suppose that the rewriting system $\mathcal{Z}$ is not locally confluent. Then there exist elements which are not locally confluent. Since $\prec$ is a well-ordering, there exists a least element $W \in \mathcal{S}(\mathcal{X}, \Phi)$ which is not locally confluent. As each proper $\Phi$-subword $U$ of $W$ satisfies $U \prec W$, each proper $\Phi$-subword of $W$ is locally confluent. Therefore, the Critical Pair Lemma 5.17 applies to $W$ : There exist rewriting rules $P_{i} \rightarrow Q_{i}$ and $P_{j} \rightarrow Q_{j}$ in the $\Phi$-generating set $\mathcal{Y}$ of the rewriting system $\mathcal{Z}$ so that either one of the following conditions hold:

(i) $W=P_{1}$ and $W=A P_{2}^{\sigma} B$ for $\sigma \in \Phi^{*}$ and $A, B \in \mathcal{S}(\mathcal{X}, \Phi)$.

(ii) $W=P_{1}^{\sigma}$ and $W=A^{\sigma} P_{2} B^{\sigma}$ for $\sigma \in \Phi^{*}$ and non-empty words $A, B \in \mathcal{S}(\mathcal{X}, \Phi)$.

(iii) $W$ can be written $(A B)^{\sigma} C$ or $A(B C)^{\sigma}$ for non-empty words $A, B, C \in \mathcal{S}(\mathcal{X}, \Phi)$, $\sigma \in \Phi^{*}$, and either

- $P_{1}=A B$ and $P_{2}=B^{\sigma} C$, or

- $P_{1}=A B^{\sigma}$ and $P_{2}=B C$.

Suppose that either one of these conditions holds. Since the algorithm LKnUthBEndix ensures that all overlaps are checked, the rules $P_{i} \rightarrow Q_{i}$ and $P_{j} \rightarrow Q_{j}$ would have been checked for an overlap of this type and it would have enforced local confluence of $W$ by adding an appropriate rewriting rule. Therefore, the rewriting system $\mathcal{Z}$ with $\Phi$ generating set $\mathcal{Y}$ is locally confluent and it generates the monoid congruence $\sim$. As $\mathcal{Z}$ is Noetherian by construction, $\mathcal{Z}$ is confluent and, by Corollary 5.3 , it allows to rewrite each $W \in \mathcal{S}(\mathcal{X}, \Phi)$ to its canonical form.

Suppose that there exists a confluent $\Phi$-finite rewriting system $\mathcal{T}$ with finite $\Phi$ generating set $\mathcal{R}$ for the monoid in Theorem 5.19. Then the rewriting systems $\mathcal{T}$ and 
$\mathcal{Z}$ both induce the same monoid congruence $\sim$ which is generated by the monoid $L$ presentation in Theorem 5.19, The reduction ordering $\prec$ on $\mathcal{S}(\mathcal{X}, \Phi)$ allows us to define the set of canonical forms

$$
\mathcal{C}=\{P \in \mathcal{S}(\mathcal{X}, \Phi) \mid \text { for each } U \in \mathcal{S}(\mathcal{X}, \Phi) \text { we have } U \sim P \Rightarrow U \succeq P\} .
$$

This definition does not depend on the rewriting systems $\mathcal{T}$ and $\mathcal{Z}$. Let $\mathcal{P}$ be the set of elements $W \in \mathcal{S}(\mathcal{X}, \Phi) \backslash \mathcal{C}$ so that each proper $\Phi$-subword of $W$ is a canonical form. Let $W \in \mathcal{P}$ be given. Since each proper $\Phi$-subword of $W$ is a canonical form, it is irreducible by Lemma 5.2. Because the confluent rewriting systems $\mathcal{T}$ and $\mathcal{Z}$ allow us to rewrite the element $W$ to its canonical form, both rewriting systems contain a (unique) rewriting rule with left-side $W$. Since $\mathcal{R}$ is a $\Phi$-generating set for the rewriting system $\mathcal{T}$, there exists a (unique) rewriting rule $P \rightarrow Q$ in $\mathcal{R}$ and $\psi \in \Phi^{*}$ so that $W=P^{\psi}$. If $\psi$ is non-trivial, $P$ is a proper subword of $W$ and thus $P \in \mathcal{C}$; this, however, contradicts the existence of a rewriting rule $P \rightarrow Q$ which implies that $P \succ Q$ holds. Therefore, $W=P$ is actually contained in the $\Phi$-generating set $\mathcal{R}$. In particular, the set of rewriting rules $\{P \rightarrow Q \mid P \in \mathcal{P}\} \subseteq \mathcal{R}$ is finite and so is $\mathcal{P}$.

Consider the $\Phi$-generating set $\mathcal{Y}$ with the rewriting rules $P_{i} \rightarrow Q_{i}$ that were generated by the algorithm LKnuthBendix. Since $\mathcal{P}$ is finite, there exists a positive integer $n$ that is maximal subject to $P_{n} \in \mathcal{P}$. Let $i>n$ be given. The elements $P_{i}$ and $Q_{i}$ are, by construction, irreducible with respect to $\mathcal{Z}_{i}=\left\{P_{j}^{\psi} \rightarrow Q_{j}^{\psi} \mid 1 \leq j<i, \psi \in \Phi^{*}\right\}$. However, $P_{i} \notin \mathcal{P}$ and $P_{i} \notin \mathcal{C}$. Therefore, $P_{i}$ must contain a proper $\Phi$-subword $U$ that is contained in $\mathcal{P}$. But this is impossible as either $\mathcal{Z}$ does not allow to rewrite $U$ to its canonical form (contradicting Corollary 5.3) or $U$ must be the left-side of a rule in $\left\{P_{j} \rightarrow Q_{j} \mid 1 \leq j \leq n\right\}$ (contradicting irreducibility of $P_{i}$ with respect to $\mathcal{Z}_{i}$ ). Therefore, the algorithm LKNUthBEndix cannot produce an infinite $\Phi$-generating set $\mathcal{Y}$. It terminates and computes a finite $\Phi$-generating set for a confluent $\Phi$-finite rewriting system.

It should be emphasized here that Theorem 5.19 does not imply that there is a procedure that allows to decide whether or not a group admits a $\Phi$-finite rewriting system. The proof of Theorem 5.19 also yields the following

Corollary 5.20 For a finite group $G$, the algorithm LKnUthBendix terminates and it computes a finite $\Phi$-generating set for a confluent rewriting system for $G$.

Proof. If $G$ is finite, there are only finitely many canonical forms. In particular, for a rewriting system $\mathcal{T}$ with $\Phi$-generating set $\mathcal{R}$ and a monoid congruence $\sim$, the set of canonical forms $\mathcal{C}$ defined in the proof of Theorem 5.19 is finite. It suffices to prove finiteness of the set $\mathcal{P}$ of elements $W \in \mathcal{S}(\mathcal{X}, \Phi) \backslash \mathcal{C}$ so that each proper $\Phi$-subword of $W$ is contained in $\mathcal{C}$. If this is the case, the proof of Theorem 5.19 applies and it would prove that the algorithm LKNUTHBENDIX terminates.

Recall that the elements $W \in \mathcal{S}(\mathcal{X}, \Phi)$ have the form $W=x_{1}^{\sigma_{1}} \cdots x_{n}^{\sigma_{n}}$. Since $\mathcal{C}$ is finite, there exists a positive integer $\ell$ that is maximal subject to $x_{1}^{\sigma_{1}} \cdots x_{\ell}^{\sigma_{\ell}} \in \mathcal{C}$. Let $V \in \mathcal{P}$ be given. Then each proper $\Phi$-subword of $V$ is contained in $\mathcal{C}$. If $V=x_{1}^{\sigma_{1}} \cdots x_{n}^{\sigma_{n}}$ 
for $n>\ell+1$, the $\Phi$-subword $U=x_{1}^{\sigma_{1}} \cdots x_{\ell+1}^{\sigma_{\ell+1}}$ of $V$ is proper and it is not contained in $\mathcal{C}$. Therefore, $V \in \mathcal{P}$ has the form $V=x_{1}^{\sigma_{1}} \cdots x_{n}^{\sigma_{n}}$ with $n \leq \ell+1$.

Similar, as $\mathcal{C}$ is finite, there exists a positive integer $m$ that is maximal subject to $x_{1}^{\sigma_{1}} \cdots x_{n}^{\sigma_{n}} \in \mathcal{C}$ with each $\left\|\sigma_{i}\right\| \leq m$. Let $V \in \mathcal{P}$ be given. Then $V=x_{1}^{\sigma_{1}} \cdots x^{\sigma_{n}}$ with $n \leq \ell+1$. Suppose that for some $1 \leq i \leq n$ we have that $\left\|\sigma_{i}\right\|=k>m$. Then $\sigma=\delta_{1} \cdots \delta_{m} \delta_{m+1} \cdots \delta_{k}$ with each $\delta_{i} \in \Phi$. Then $x_{i}^{\delta_{1} \cdots \delta_{m+1}}$ is a proper $\Phi$-subword of $V$ but $x_{i}^{\delta_{1} \cdots \delta_{m+1}} \notin \mathcal{C}$.

It follows that the elements $V \in \mathcal{P}$ are 'bounded' so that there are only finitely many such elements. Since $\mathcal{P}$ is finite, the ideas of the proof of Theorem 5.19 apply. These show that algorithm LKNUTHBEndix terminates and it computes a finite $\Phi$-generating set for the rewriting system for $G$.

\subsection{An Application of the Knuth-Bendix Approach}

In order to illustrate our Knuth-Bendix approach for invariantly finitely $L$-presented groups, we consider a fairly easy $L$-presentation so that (most) computations can be done by hand. Consider the following ascending finite $L$-presentation

$$
\left\langle\left\{a_{12}, a_{13}, a_{14}, a_{23}, a_{24}, a_{34}\right\}|\emptyset|\{\sigma, \delta\} \mid\left\{a_{12}^{2}, a_{12} a_{23} a_{13} a_{23}\right\}\right\rangle
$$

where the endomorphisms $\sigma$ and $\delta$ are induced by the maps

$$
\sigma:\left\{\begin{aligned}
a_{12} & \mapsto a_{12}, \\
a_{13} & \mapsto a_{23}, \\
a_{14} & \mapsto a_{24}, \\
a_{23} & \mapsto a_{13}, \\
a_{24} & \mapsto a_{14}, \\
a_{34} & \mapsto a_{34},
\end{aligned} \quad \text { and } \quad \delta:\left\{\begin{aligned}
a_{12} & \mapsto a_{23}, \\
a_{13} & \mapsto a_{24}, \\
a_{14} & \mapsto a_{12}, \\
a_{23} & \mapsto a_{34}, \\
a_{24} & \mapsto a_{13}, \\
a_{34} & \mapsto a_{14} .
\end{aligned}\right.\right.
$$

The finite $L$-presentation in Eq. (5.10) is a finite $L$-presentation for the symmetric group $\mathcal{S}_{4}$ over four letters [6]. Each generator $a_{i j}$ corresponds to the transposition $(i, j) \in \mathcal{S}_{4}$ and the endomorphisms $\sigma$ and $\delta$ correspond to the inner automorphisms that are induced by conjugation with $(1,2)$ and $(1,2,3,4)$, respectively. From the finite $L$-presentation in Eq. (5.10) we obtain the following relators:

$$
a_{12}^{2}, a_{13}^{2}=\left(a_{12}^{2}\right)^{\delta \sigma}, a_{14}^{2}=\left(a_{12}^{2}\right)^{\delta \delta \delta}, a_{23}^{2}=\left(a_{12}^{2}\right)^{\delta}, a_{24}^{2}=\left(a_{12}^{2}\right)^{\delta \sigma \delta}, a_{34}^{2}=\left(a_{12}^{2}\right)^{\delta \delta} .
$$

In particular, each generator $a_{i j}$ is either trivial or it has order 2. Thus these generators satisfy $a_{i j}^{-1}=a_{i j}$. Therefore, we can consider the group presentation in Eq. (5.10) as a monoid $L$-presentation for $\mathcal{S}_{4}$. Otherwise, we would need to consider the formal inverses $\mathcal{X}^{-}=\left\{a_{12}^{-1}, a_{13}^{-1}, a_{14}^{-1}, a_{23}^{-1}, a_{24}^{-1}, a_{34}^{-1}\right\}$ together with the relations

$$
\varepsilon=a_{12}^{-1} a_{12}=a_{12} a_{12}^{-1}=a_{13}^{-1} a_{13}=a_{13} a_{13}^{-1}=\ldots=a_{34}^{-1} a_{34}=a_{34} a_{34}^{-1}
$$

and extensions $\tilde{\sigma}$ and $\tilde{\delta}$ of the endomorphisms $\sigma$ and $\delta$ as in Proposition 5.5. 
We choose the orderings $\sigma \prec \delta$ and $a_{12} \prec a_{13} \prec a_{14} \prec a_{23} \prec a_{24} \prec a_{34}$ on the monoid generators $\Phi$ and $\mathcal{X}$, respectively. These orderings extend to a reduction ordering $\prec$ on

$$
\mathcal{S}(\mathcal{X}, \Phi)=\left\{x_{1}^{\sigma_{1}} \cdots x_{n}^{\sigma_{n}} \mid x_{i} \in\left\{a_{12}, a_{13}, a_{14}, a_{23}, a_{24}, a_{34}\right\}, \sigma_{i} \in\{\sigma, \delta\}^{*}, 1 \leq i \leq n\right\}
$$

as described in Section 5.3. The reduction ordering $\prec$ yields the rewriting rules

$$
\begin{array}{llllll}
a_{12}^{\sigma} \rightarrow a_{12}, & a_{23}^{\sigma} \rightarrow a_{13}, & a_{23} \rightarrow a_{12}^{\delta}, & a_{34} \rightarrow a_{23}^{\delta}, \\
a_{23} \rightarrow a_{13}^{\sigma}, & a_{24}^{\sigma} \rightarrow a_{14}, & a_{24} \rightarrow a_{13}^{\delta}, & a_{24}^{\delta} \rightarrow a_{13}, \\
a_{24} \rightarrow a_{14}^{\sigma}, & a_{34}^{\sigma} \rightarrow a_{34}, & a_{14}^{\delta} \rightarrow a_{12}, & a_{34}^{\delta} \rightarrow a_{14} .
\end{array}
$$

as well as

$$
a_{12} a_{12} \rightarrow \varepsilon \quad \text { and } \quad a_{12} a_{23} a_{13} a_{23} \rightarrow \varepsilon .
$$

We first reduce the rewriting system. For this purpose, we consider the rewriting rules one after another and we reduce overlaps of the form (i) and (ii) in the Critical Pair Lemma 5.17, i.e., rewriting rules $P_{1} \rightarrow Q_{1}$ and $P_{2} \rightarrow Q_{2}$ so that $P_{1}^{\omega}$, for $\omega \in \Phi^{*}$, contains $P_{2}$ as a proper $\Phi$-subword. We always try to keep the rewriting system reduced. There are no overlaps of type (i) or (ii) among the rewriting rules

$$
\begin{aligned}
& a_{12}^{\sigma} \rightarrow a_{12} \\
& a_{23} \rightarrow a_{13}^{\sigma} \\
& a_{24} \rightarrow a_{14}^{\sigma} .
\end{aligned}
$$

Consider the rewriting rule $a_{23}^{\sigma} \rightarrow a_{13}$. The left-side of this rule contains $a_{23}$ as a proper $\Phi$-subword. Thus there is an overlap with the rewriting rule $a_{23} \rightarrow a_{13}^{\sigma}$ from Eq. (5.14). This overlap yields both

$$
a_{23}^{\sigma} \rightarrow a_{13} \quad \text { and } \quad a_{23}^{\sigma}=\left(a_{23}\right)^{\sigma} \rightarrow\left(a_{13}^{\sigma}\right)^{\sigma}=a_{13}^{\sigma \sigma} .
$$

This yields the rewriting rule

$$
a_{13}^{\sigma \sigma} \rightarrow a_{13}
$$

We can omit the rewriting rule $a_{23}^{\sigma} \rightarrow a_{13}$ in the following. Similar, there is an overlap within the rewriting rules $a_{24}^{\sigma} \rightarrow a_{14}$ and $a_{24} \rightarrow a_{14}^{\sigma}$ from Eq. (5.15). This overlap yields both $a_{24}^{\sigma} \rightarrow a_{14}$ and $a_{24}^{\sigma} \rightarrow a_{14}^{\sigma \sigma}$. Hence, we obtain the rewriting rule

$$
a_{14}^{\sigma \sigma} \rightarrow a_{14}
$$

while we can omit the rule $a_{24}^{\sigma} \rightarrow a_{14}$. There are no overlaps with the rewriting rule

$$
a_{34}^{\sigma} \rightarrow a_{34} .
$$

Consider the rewriting rule $a_{23} \rightarrow a_{12}^{\delta}$. Then there is an overlap with the rule $a_{23} \rightarrow a_{13}^{\sigma}$ from Eq. (5.14). This overlap yields $a_{23} \rightarrow a_{13}^{\sigma}$ and $a_{23} \rightarrow a_{12}^{\delta}$. Since $a_{12}^{\delta} \prec a_{13}^{\sigma}$ holds, we obtain the rewriting rule

$$
a_{13}^{\sigma} \rightarrow a_{12}^{\delta} .
$$


Furthermore, we replace the rewriting rule $a_{23} \rightarrow a_{13}^{\sigma}$ in Eq. (5.14) by

$$
a_{23} \rightarrow a_{12}^{\delta} \text {. }
$$

At this stage, the rewriting system given by the rewriting rules above is not reduced anymore because the rule in Eq. (5.19) has an overlap with $a_{13}^{\sigma \sigma} \rightarrow a_{13}$ from Eq. (5.16). This overlap, on the one hand, gives $a_{13}^{\sigma \sigma} \rightarrow a_{13}$ while, on the other hand, it gives $a_{13}^{\sigma \sigma}=\left(a_{13}^{\sigma}\right)^{\sigma} \rightarrow a_{12}^{\delta \sigma}$. This yields

$$
a_{13} \rightarrow a_{12}^{\delta \sigma}
$$

We therefore replace the rule $a_{13}^{\sigma \sigma} \rightarrow a_{13}$ in Eq. (5.16) by

$$
a_{12}^{\delta \sigma \sigma \sigma} \rightarrow a_{12}^{\delta \sigma}
$$

The rule $a_{13} \rightarrow a_{12}^{\delta \sigma}$ from Eq. (5.21) has an overlap with $a_{13}^{\sigma} \rightarrow a_{12}^{\delta}$ from Eq. (5.19). This overlap gives $a_{13}^{\sigma} \rightarrow a_{12}^{\delta}$ and $a_{13}^{\sigma}=\left(a_{13}\right)^{\sigma} \rightarrow a_{12}^{\delta \sigma \sigma}$. We therefore replace the rule $a_{13}^{\sigma} \rightarrow a_{12}^{\delta}$ from Eq. (5.19) by

$$
a_{12}^{\delta \sigma \sigma} \rightarrow a_{12}^{\delta}
$$

The latter rule yields that $a_{12}^{\delta \sigma \sigma \sigma} \rightarrow a_{12}^{\delta \sigma}$ from Eq. (5.22) is redundant. We have obtained a reduced rewriting system again. Consider the rewriting rule $a_{24} \rightarrow a_{13}^{\delta} \rightarrow a_{12}^{\delta \sigma \delta}$. Then there is an overlap with the rule $a_{24} \rightarrow a_{14}^{\sigma}$ from Eq. (5.15). This overlap gives

$$
a_{14}^{\sigma} \rightarrow a_{12}^{\delta \sigma \delta}
$$

and we replace the rule $a_{24} \rightarrow a_{14}^{\sigma}$ from Eq. (5.15) by

$$
a_{24} \rightarrow a_{12}^{\delta \sigma \delta} .
$$

Again the above rewriting system is not reduced because the rule $a_{14}^{\sigma} \rightarrow a_{12}^{\delta \sigma \delta}$ from Eq. (5.24) has an overlap with $a_{14}^{\sigma \sigma} \rightarrow a_{14}$ from Eq. (5.17). This overlap gives $a_{14}^{\sigma \sigma}=$ $\left(a_{14}^{\sigma}\right)^{\sigma} \rightarrow a_{12}^{\delta \sigma \delta \sigma}$. We replace the rule $a_{14}^{\sigma \sigma} \rightarrow a_{14}$ from Eq. (5.17) by

$$
a_{14} \rightarrow a_{12}^{\delta \sigma \delta \sigma}
$$

The latter rule has an overlap with $a_{14}^{\sigma} \rightarrow a_{12}^{\delta \sigma \delta}$ from Eq. (5.24). This overlap allows us to replace $a_{14}^{\sigma} \rightarrow a_{12}^{\delta \sigma \delta}$ in Eq. (5.24) by

$$
a_{12}^{\delta \sigma \delta \sigma \sigma} \rightarrow a_{12}^{\delta \sigma \delta} .
$$

We have obtained a reduced rewriting system again. Consider the rule $a_{14}^{\delta} \rightarrow a_{12}$. Then the overlap with $a_{14} \rightarrow a_{12}^{\delta \sigma \delta \sigma}$ from Eq. (5.26) yields the rewriting rule

$$
a_{12}^{\delta \sigma \delta \sigma \delta} \rightarrow a_{12} .
$$

The rule $a_{34} \rightarrow a_{23}^{\delta} \rightarrow a_{12}^{\delta \delta}$ gives us

$$
a_{34} \rightarrow a_{12}^{\delta \delta}
$$


The latter rule has an overlap with $a_{34}^{\sigma} \rightarrow a_{34}$ from Eq. (5.18). This overlap allows us to replace the rule from Eq. (5.18) by

$$
a_{12}^{\delta \delta \sigma} \rightarrow a_{12}^{\delta \delta}
$$

Next we consider the rule $a_{24}^{\delta} \rightarrow a_{13} \rightarrow a_{12}^{\delta \sigma}$. This rule has an overlap with $a_{24} \rightarrow a_{12}^{\delta \sigma \delta}$ in Eq. (5.25). It gives us the rewriting rule

$$
a_{12}^{\delta \sigma \delta \delta} \rightarrow a_{12}^{\delta \sigma}
$$

Finally, we need to consider the rule $a_{34}^{\delta} \rightarrow a_{14} \rightarrow a_{12}^{\delta \sigma \delta \sigma}$. This latter rule has an overlap with $a_{34} \rightarrow a_{12}^{\delta \delta}$ from Eq. (5.29). This overlap gives us

$$
a_{12}^{\delta \sigma \delta \sigma} \rightarrow a_{12}^{\delta \delta \delta} .
$$

This rule has various overlaps with the other rewriting rules. First, we can replace the rule $a_{14} \rightarrow a_{12}^{\delta \sigma \delta \sigma}$ from Eq. (5.26) by

$$
a_{14} \rightarrow a_{12}^{\delta \delta \delta}
$$

Then the left-side of the rule $a_{12}^{\delta \sigma \delta \sigma \delta} \rightarrow a_{12}$ in Eq. (5.28) contains $a_{12}^{\delta \sigma \delta \sigma}$ as a proper $\Phi$-subword and thus we can replace the rule in Eq. (5.28) by

$$
a_{12}^{\delta \delta \delta \delta} \rightarrow a_{12}
$$

Similar, the left-side of the rule $a_{12}^{\delta \sigma \delta \sigma \sigma} \rightarrow a_{12}^{\delta \sigma \delta}$ in Eq. (5.27) contains $a_{12}^{\delta \sigma \delta \sigma}$ as a proper $\Phi$-subword. We therefore replace the rule in Eq. (5.27) by

$$
a_{12}^{\delta \delta \sigma \sigma} \rightarrow a_{12}^{\delta \sigma \delta} .
$$

So far, we have obtained a reduced rewriting system whose rewriting rules stem from the substitutions $\sigma$ and $\delta$ in Eq. (5.11). It remains to consider the rewriting rules which stem from the iterated relations of the $L$-presentation in Eq. (5.11). The first rule indicates that the generator $a_{12}$ is an involution

$$
a_{12} a_{12} \rightarrow \varepsilon
$$

This latter rule has several overlaps with the second rule $a_{12} a_{23} a_{13} a_{23} \rightarrow \varepsilon$ from Eq. (5.11). The rewriting rules above yield that

$$
a_{12} a_{23} a_{13} a_{23} \rightarrow a_{12} a_{12}^{\delta} a_{12}^{\delta \sigma} a_{12}^{\delta} \rightarrow \varepsilon
$$

At this stage, we have obtained a reduced rewriting system for the finitely $L$-presented group in Eq. (5.10). We have proved 
Proposition 5.21 For the symmetric group on four letters with its L-presentation from Eq. (5.10) and the orderings $\sigma \prec \delta$ and $a_{12} \prec a_{13} \prec a_{14} \prec a_{23} \prec a_{24} \prec a_{34}$, a reduced $\Phi$-finite rewriting system is given by the following rewriting rules:

$$
\begin{aligned}
a_{12}^{\sigma} & \rightarrow a_{12}, & a_{13} & \rightarrow a_{12}^{\delta \sigma}, & a_{14} & \rightarrow a_{12}^{\delta \delta \delta}, \\
a_{23} & \rightarrow a_{12}^{\delta}, & a_{24} & \rightarrow a_{12}^{\delta \sigma \delta}, & a_{34} & \rightarrow a_{12}^{\delta \delta}, \\
a_{12}^{\delta \sigma \sigma} & \rightarrow a_{12}^{\delta}, & a_{12}^{\delta \delta \sigma} & \rightarrow a_{12}^{\delta \delta}, & a_{12}^{\delta \sigma \delta \sigma} & \rightarrow a_{12}^{\delta \delta \delta}, \\
a_{12}^{\delta \sigma \delta \delta} & \rightarrow a_{12}^{\delta \sigma}, & a_{12}^{\delta \delta \sigma} & \rightarrow a_{12}^{\delta \sigma \delta}, & a_{12}^{\delta \delta \delta \delta} & \rightarrow a_{12},
\end{aligned}
$$

and

$$
a_{12} a_{12} \rightarrow \varepsilon \quad \text { and } \quad a_{12} a_{12}^{\delta} a_{12}^{\delta \sigma} a_{12}^{\delta} \rightarrow \varepsilon .
$$

In the following, we enforce local confluence of this rewriting system by considering the overlaps of type (iii) in the Critical Pair Lemma 5.17. We indicate which rewriting rule is applied first by underlining the appropriate $\Phi$-subword. The rewriting rules in Eq. (5.39) overlap as follows:

$$
\underline{a_{12} a_{12}} a_{12}^{\delta} a_{12}^{\delta \sigma} a_{12}^{\delta} \rightarrow a_{12}^{\delta} a_{12}^{\delta \sigma} a_{12}^{\delta} \quad \text { and } \quad a_{12} \underline{a_{12} a_{12}^{\delta} a_{12}^{\delta \sigma} a_{12}^{\delta}} \rightarrow a_{12} .
$$

as well as

$$
a_{12} a_{12}^{\delta} a_{12}^{\delta \sigma} \underline{a_{12}^{\delta} a_{12}^{\delta}} \rightarrow a_{12} a_{12}^{\delta} a_{12}^{\delta \sigma} \quad \text { and } \quad \underline{a_{12} a_{12}^{\delta} a_{12}^{\delta \sigma} a_{12}^{\delta}} a_{12}^{\delta} \rightarrow a_{12}^{\delta} .
$$

These overlaps give

$$
a_{12}^{\delta} a_{12}^{\delta \sigma} a_{12}^{\delta} \rightarrow a_{12} \quad \text { and } \quad a_{12} a_{12}^{\delta} a_{12}^{\delta \sigma} \rightarrow a_{12}^{\delta} .
$$

Again, overlaps with the rule $a_{12} a_{12} \rightarrow \varepsilon$ from Eq. (5.36) give

$$
\begin{aligned}
& a_{12}^{\delta} a_{12}^{\delta} a_{12}^{\delta \sigma} a_{12}^{\delta} \rightarrow a_{12}^{\delta \sigma} a_{12}^{\delta} \quad \text { and } \quad a_{12}^{\delta} a_{12}^{\delta} a_{12}^{\delta \sigma} a_{12}^{\delta} \rightarrow a_{12}^{\delta} a_{12} \text {, } \\
& \overline{a_{12}^{\delta} a_{12}^{\delta \sigma}} a_{12}^{\delta} a_{12}^{\delta} \rightarrow a_{12} a_{12}^{\delta} \quad \text { and } a_{12}^{\delta} \overline{a_{12}^{\delta \sigma} \frac{a_{12}^{\delta} a_{12}^{\delta}}{a_{12}}} \rightarrow a_{12}^{\delta} a_{12}^{\delta \sigma} \text {, } \\
& \underline{a_{12} a_{12}} a_{12}^{\delta} a_{12}^{\delta \sigma} \rightarrow a_{12}^{\delta} a_{12}^{\delta \sigma} \quad \text { and } \quad a_{12} \underline{a_{12}} \overline{a_{12}^{\delta} a_{12}^{\delta \sigma}} \rightarrow a_{12} a_{12}^{\delta} \text {, } \\
& \underline{a_{12} a_{12}^{\delta}} a_{12}^{\delta \sigma} a_{12}^{\delta \sigma} \rightarrow a_{12}^{\delta} a_{12}^{\delta \sigma} \quad \text { and } a_{12} \overline{a_{12}^{\delta} \underline{a_{12}^{\delta \sigma} a_{12}^{\delta \sigma}}} \rightarrow a_{12} a_{12}^{\delta} \text {. }
\end{aligned}
$$

Therefore, we obtain the rules

$$
a_{12}^{\delta} a_{12}^{\delta \sigma} \rightarrow a_{12} a_{12}^{\delta}
$$

and

$$
a_{12}^{\delta \sigma} a_{12}^{\delta} \rightarrow a_{12}^{\delta} a_{12} .
$$

Consider the overlap of the rule $a_{12}^{\delta \delta \delta \delta} \rightarrow a_{12}$ from Eq. (5.34) with the rule from Eq. (5.41).

This overlap gives us

$$
a_{12}^{\delta \delta \delta \delta} a_{12}^{\delta \sigma \delta \delta \delta} \rightarrow a_{12} a_{12}^{\delta \sigma \delta \delta \delta} \rightarrow^{*} a_{12} a_{12}^{\delta \sigma \delta}
$$

and

$$
a_{12}^{\delta \delta \delta \delta} a_{12}^{\delta \sigma \delta \delta \delta}=\left(a_{12}^{\delta} a_{12}^{\delta \sigma}\right)^{\delta \delta \delta} \rightarrow\left(a_{12} a_{12}^{\delta}\right)^{\delta \delta \delta} \rightarrow^{*} a_{12}^{\delta \delta \delta} a_{12}
$$


Hence, we obtain the rewriting rule

$$
a_{12}^{\delta \delta \delta} a_{12} \rightarrow a_{12} a_{12}^{\delta \sigma \delta} .
$$

Similar, the overlap of $a_{12}^{\delta \delta \delta \delta} \rightarrow a_{12}$ from Eq. (5.34) and $a_{12}^{\delta \sigma} a_{12}^{\delta} \rightarrow a_{12}^{\delta} a_{12}$ from Eq. (5.42) gives

$$
a_{12}^{\delta \sigma \delta \delta \delta} a_{12}^{\delta \delta \delta \delta} \rightarrow a_{12}^{\delta \sigma \delta \delta \delta} a_{12} \rightarrow^{*} a_{12}^{\delta \sigma \delta} a_{12} \quad \text { and } \quad a_{12}^{\delta \sigma \delta \delta \delta} a_{12}^{\delta \delta \delta \delta} \rightarrow a_{12}^{\delta \delta \delta \delta} a_{12}^{\delta \delta \delta} \rightarrow^{*} a_{12} a_{12}^{\delta \delta \delta} .
$$

This yields the rewriting rule

$$
a_{12}^{\delta \sigma \delta} a_{12} \rightarrow a_{12} a_{12}^{\delta \delta \delta} .
$$

An overlap of $a_{12}^{\delta \delta \sigma} \rightarrow a_{12}^{\delta \delta}$ from Eq. (5.30) and $a_{12}^{\delta} a_{12}^{\delta \sigma} \rightarrow a_{12} a_{12}^{\delta}$ from Eq. (5.41) gives

$$
a_{12}^{\delta \delta \sigma} a_{12}^{\delta \sigma \delta \sigma} \rightarrow a_{12}^{\delta \delta} a_{12}^{\delta \sigma \delta \sigma} \rightarrow^{*} a_{12}^{\delta \delta} a_{12}^{\delta \delta \delta} \quad \text { and } \quad a_{12}^{\delta \delta \sigma} a_{12}^{\delta \sigma \delta \sigma} \rightarrow a_{12}^{\delta \sigma} a_{12}^{\delta \delta \sigma} \rightarrow^{*} a_{12}^{\delta \sigma} a_{12}^{\delta \delta} .
$$

We obtain the rewriting rule

$$
a_{12}^{\delta \delta} a_{12}^{\delta \delta \delta} \rightarrow a_{12}^{\delta \sigma} a_{12}^{\delta \delta} .
$$

Consider the overlap of $a_{12}^{\delta \delta \delta \sigma} \rightarrow a_{12}^{\delta \sigma \delta}$ from Eq. (5.35) and $a_{12}^{\delta} a_{12}^{\delta \sigma} \rightarrow a_{12} a_{12}^{\delta}$ from Eq. (5.41). Then, on the one hand, we obtain

$$
a_{12}^{\delta \delta \sigma} a_{12}^{\delta \sigma \delta \delta \sigma} \rightarrow a_{12}^{\delta \sigma \delta} a_{12}^{\delta \sigma \delta \delta \sigma} \rightarrow a_{12}^{\delta \sigma \delta} a_{12}^{\delta \sigma \sigma} \rightarrow a_{12}^{\delta \sigma \delta} a_{12}^{\delta}
$$

while, on the other hand, we obtain

$$
a_{12}^{\delta \delta \sigma} a_{12}^{\delta \sigma \delta \delta \sigma} \rightarrow a_{12}^{\delta \delta \sigma} a_{12}^{\delta \delta \delta \sigma} \rightarrow a_{12}^{\delta \delta \sigma} a_{12}^{\delta \sigma \delta} \rightarrow a_{12}^{\delta \delta} a_{12}^{\delta \sigma \delta}=\left(a_{12}^{\delta} a_{12}^{\delta \sigma}\right)^{\delta} \rightarrow a_{12}^{\delta} a_{12}^{\delta \delta} .
$$

This yields the rewriting rule

$$
a_{12}^{\delta \sigma \delta} a_{12}^{\delta} \rightarrow a_{12}^{\delta} a_{12}^{\delta \delta} .
$$

Consider an overlap of the latter rule with the rule $a_{12}^{\delta \delta \delta \delta} \rightarrow a_{12}$ from Eq. (5.34). This overlap gives us

$$
a_{12}^{\delta \sigma \delta \delta \delta \delta} a_{12}^{\delta \delta \delta \delta} \rightarrow a_{12}^{\delta \sigma \delta \delta} a_{12} \rightarrow a_{12}^{\delta \sigma} a_{12}
$$

and

$$
a_{12}^{\delta \sigma \delta \delta \delta \delta} a_{12}^{\delta \delta \delta \delta} \rightarrow a_{12}^{\delta \delta \delta \delta} a_{12}^{\delta \delta \delta \delta \delta} \rightarrow^{*} a_{12} a_{12}^{\delta} .
$$

Whence $a_{12}^{\delta \sigma} a_{12} \rightarrow a_{12} a_{12}^{\delta}$ and we can replace the rule in Eq. (5.46) by

$$
a_{12}^{\delta \sigma} a_{12} \rightarrow a_{12} a_{12}^{\delta} .
$$

An overlap of the latter rule with $a_{12}^{\delta \sigma \sigma} \rightarrow a_{12}^{\delta}$ from Eq. (5.23) gives

$$
a_{12}^{\delta \sigma \sigma} a_{12}^{\sigma} \rightarrow a_{12}^{\delta} a_{12}^{\sigma} \rightarrow a_{12}^{\delta} a_{12} \quad \text { and } \quad a_{12}^{\delta \sigma \sigma} a_{12}^{\sigma} \rightarrow a_{12}^{\sigma} a_{12}^{\delta \sigma} \rightarrow a_{12} a_{12}^{\delta \sigma} .
$$

Therefore, we obtain the rewriting rule

$$
a_{12}^{\delta} a_{12} \rightarrow a_{12} a_{12}^{\delta \sigma} .
$$


Note that we can replace the rewriting rule $a_{12}^{\delta \sigma} a_{12}^{\delta} \rightarrow a_{12}^{\delta} a_{12}$ in Eq. (5.42) by

$$
a_{12}^{\delta \sigma} a_{12}^{\delta} \rightarrow a_{12} a_{12}^{\delta \sigma} .
$$

The overlap of $a_{12}^{\delta} a_{12} \rightarrow a_{12} a_{12}^{\delta \sigma}$ from Eq. (5.48) with $a_{12}^{\delta \sigma \delta \sigma} \rightarrow a_{12}^{\delta \delta \delta}$ from Eq. (5.32) yields

$$
a_{12}^{\delta \sigma \delta \sigma} a_{12}^{\sigma \delta \sigma} \rightarrow a_{12}^{\delta \delta \delta} a_{12}^{\sigma \delta \sigma} \rightarrow a_{12}^{\delta \delta \delta} a_{12}^{\delta \sigma} \quad \text { and } \quad a_{12}^{\delta \sigma \delta \sigma} a_{12}^{\sigma \delta \sigma} \rightarrow a_{12}^{\sigma \delta \sigma} a_{12}^{\delta \sigma \sigma \delta \sigma} \rightarrow^{*} a_{12}^{\delta \sigma} a_{12}^{\delta \delta} .
$$

This yields the rewriting rule

$$
a_{12}^{\delta \delta \delta} a_{12}^{\delta \sigma} \rightarrow a_{12}^{\delta \sigma} a_{12}^{\delta \delta} .
$$

An overlap of $a_{12}^{\delta} a_{12} \rightarrow a_{12} a_{12}^{\delta \sigma}$ from Eq. (5.48) with $a_{12}^{\delta \delta \sigma} \rightarrow a_{12}^{\delta \delta}$ from Eq. (5.30) yields

$$
a_{12}^{\delta \delta \sigma} a_{12}^{\delta \sigma} \rightarrow a_{12}^{\delta \delta} a_{12}^{\delta \sigma} \quad \text { and } \quad a_{12}^{\delta \delta \sigma} a_{12}^{\delta \sigma} \rightarrow a_{12}^{\delta \sigma} a_{12}^{\delta \sigma \delta \sigma} \rightarrow a_{12}^{\delta \sigma} a_{12}^{\delta \delta \delta} .
$$

Whence

$$
a_{12}^{\delta \delta} a_{12}^{\delta \sigma} \rightarrow a_{12}^{\delta \sigma} a_{12}^{\delta \delta \delta} .
$$

We now consider an overlap of $a_{12}^{\delta \sigma} a_{12}^{\delta} \rightarrow a_{12} a_{12}^{\delta \sigma}$ from Eq. (5.49) and $a_{12}^{\delta \delta \delta} a_{12} \rightarrow a_{12} a_{12}^{\delta \sigma \delta}$ from Eq. (5.43). This overlaps yields that

$$
a_{12}^{\delta \sigma \delta \delta} a_{12}^{\delta \delta \delta} a_{12} \rightarrow a_{12}^{\delta \sigma \delta \delta} a_{12} a_{12}^{\delta \sigma \delta} \rightarrow a_{12}^{\delta \sigma} a_{12} a_{12}^{\delta \sigma \delta} \rightarrow a_{12} a_{12}^{\delta} a_{12}^{\delta \sigma \delta}
$$

and

$$
a_{12}^{\delta \sigma \delta \delta} a_{12}^{\delta \delta \delta} a_{12} \rightarrow a_{12}^{\delta \delta} a_{12}^{\delta \sigma \delta \delta} a_{12} \rightarrow a_{12}^{\delta \delta} a_{12}^{\delta \sigma} a_{12} \rightarrow a_{12}^{\delta \delta} a_{12} a_{12}^{\delta}
$$

We obtain the rule

$$
a_{12}^{\delta \delta} a_{12} a_{12}^{\delta} \rightarrow a_{12} a_{12}^{\delta} a_{12}^{\delta \sigma \delta} .
$$

Overlapping the latter rule with $a_{12} a_{12} \rightarrow \varepsilon$ yields that

$$
a_{12}^{\delta \delta} a_{12} \underline{a_{12}^{\delta} a_{12}^{\delta}} \rightarrow a_{12}^{\delta \delta} a_{12}
$$

and

$$
\underline{a_{12}^{\delta \delta} a_{12} a_{12}^{\delta}} a_{12}^{\delta} \rightarrow a_{12} a_{12}^{\delta} a_{12}^{\delta \sigma \delta} a_{12}^{\delta} \rightarrow a_{12} a_{12}^{\delta} a_{12}^{\delta} a_{12}^{\delta \delta} \rightarrow a_{12} a_{12}^{\delta \delta} .
$$

Whence

$$
a_{12}^{\delta \delta} a_{12} \rightarrow a_{12} a_{12}^{\delta \delta}
$$

This rewriting rule allows us to remove the rule $a_{12}^{\delta \delta} a_{12} a_{12}^{\delta} \rightarrow a_{12} a_{12}^{\delta} a_{12}^{\delta \sigma \delta}$ from Eq. (5.52) since

$$
a_{12}^{\delta \delta} a_{12} a_{12}^{\delta} \rightarrow a_{12} a_{12}^{\delta \delta} a_{12}^{\delta} \rightarrow a_{12} a_{12}^{\delta} a_{12}^{\delta \sigma \delta}
$$

holds. An overlap of the rule in Eq. (5.53) with the rule $a_{12}^{\delta \delta \delta \sigma} \rightarrow a_{12}^{\delta \sigma \delta}$ from Eq. (5.35) gives us

$$
a_{12}^{\delta \delta \sigma} a_{12}^{\delta \sigma} \rightarrow a_{12}^{\delta \sigma \delta} a_{12}^{\delta \sigma} \quad \text { and } \quad a_{12}^{\delta \delta \delta \sigma} a_{12}^{\delta \sigma} \rightarrow a_{12}^{\delta \sigma} a_{12}^{\delta \delta \delta \sigma} \rightarrow a_{12}^{\delta \sigma} a_{12}^{\delta \sigma \delta}
$$

Thus

$$
a_{12}^{\delta \sigma \delta} a_{12}^{\delta \sigma} \rightarrow a_{12}^{\delta \sigma} a_{12}^{\delta \sigma \delta} .
$$


Finally, we consider an overlap of $a_{12}^{\delta \delta} a_{12}^{\delta \delta \delta} \rightarrow a_{12}^{\delta \sigma} a_{12}^{\delta \delta}$ from Eq. (5.45) and $a_{12}^{\delta \delta \delta \delta} \rightarrow a_{12}$ from Eq. (5.34). This overlap yields

$$
a_{12}^{\delta \delta \delta} a_{12}^{\delta \delta \delta \delta} \rightarrow a_{12}^{\delta \delta \delta} a_{12} \rightarrow a_{12} a_{12}^{\delta \sigma \delta} \quad \text { and } \quad a_{12}^{\delta \delta \delta} a_{12}^{\delta \delta \delta \delta} \rightarrow a_{12}^{\delta \sigma \delta} a_{12}^{\delta \delta \delta}
$$

and we obtain the rewriting rule

$$
a_{12}^{\delta \sigma \delta} a_{12}^{\delta \delta \delta} \rightarrow a_{12} a_{12}^{\delta \sigma \delta} .
$$

With a GAP-program (or even by hand) it can be shown that all 214 overlaps of the rewriting system constructed above are satisfied so that it is (locally) confluent by the Critical Pair Lemma 5.17. This proves the following

Theorem 5.22 A reduced $\Phi$-finite confluent rewriting system for the symmetric group on four letters with respect to the reduction ordering that is induced by $\sigma \prec \delta$ and $a_{12} \prec$ $a_{13} \prec a_{14} \prec a_{23} \prec a_{24} \prec a_{34}$ is given by the $\Phi$-generating set

$$
\begin{aligned}
a_{12}^{\sigma} & \rightarrow a_{12}, & a_{13} & \rightarrow a_{12}^{\delta \sigma}, & a_{14} & \rightarrow a_{12}^{\delta \delta \delta}, \\
a_{23} & \rightarrow a_{12}^{\delta}, & a_{24} & \rightarrow a_{12}^{\delta \sigma \delta}, & a_{34} & \rightarrow a_{12}^{\delta \delta}, \\
a_{12}^{\delta \sigma \sigma} & \rightarrow a_{12}^{\delta}, & a_{12}^{\delta \delta \sigma} & \rightarrow a_{12}^{\delta \delta}, & a_{12}^{\delta \sigma \delta \sigma} & \rightarrow a_{12}^{\delta \delta \delta}, \\
a_{12}^{\delta \sigma \delta \delta} & \rightarrow a_{12}^{\delta \sigma}, & a_{12}^{\delta \delta \delta \sigma} & \rightarrow a_{12}^{\delta \sigma \delta}, & a_{12}^{\delta \delta \delta \delta} & \rightarrow a_{12},
\end{aligned}
$$

and

$$
\begin{array}{rlrlrl}
a_{12} a_{12} & \rightarrow \varepsilon, & a_{12}^{\delta} a_{12} & \rightarrow a_{12} a_{12}^{\delta \sigma}, & a_{12}^{\delta} a_{12}^{\delta \sigma} & \rightarrow a_{12} a_{12}^{\delta}, \\
a_{12}^{\delta \sigma} a_{12} & \rightarrow a_{12} a_{12}^{\delta}, & a_{12}^{\delta \sigma} a_{12}^{\delta} \rightarrow a_{12} a_{12}^{\delta \sigma}, & a_{12}^{\delta \delta} a_{12} \rightarrow a_{12} a_{12}^{\delta \delta}, \\
a_{12}^{\delta \delta} a_{12}^{\delta \sigma} \rightarrow a_{12}^{\delta \sigma} a_{12}^{\delta \delta \delta}, & a_{12}^{\delta \delta} a_{12}^{\delta \delta \delta} \rightarrow a_{12}^{\delta \sigma} a_{12}^{\delta \delta}, & a_{12}^{\delta \sigma \delta} a_{12} \rightarrow a_{12} a_{12}^{\delta \delta \delta}, \\
a_{12}^{\delta \sigma \delta} a_{12}^{\delta \sigma} \rightarrow a_{12}^{\delta \sigma} a_{12}^{\delta \sigma \delta}, & a_{12}^{\delta \sigma \delta} a_{12}^{\delta \delta \delta} \rightarrow a_{12} a_{12}^{\delta \sigma \delta}, & a_{12}^{\delta \delta \delta} a_{12} \rightarrow a_{12} a_{12}^{\delta \sigma \delta}, \\
a_{12}^{\delta \delta \delta} a_{12}^{\delta \sigma} \rightarrow a_{12}^{\delta \sigma} a_{12}^{\delta \delta} . & & & &
\end{array}
$$

An implementation of this Knuth-Bendix completion algorithm in the computer algebra system GAP [50] does not terminate for the Grigorchuk group [53], the Basilica group [61, the lamplighter group $\mathbb{Z}_{2} \imath \mathbb{Z}$ and the wreath product $\mathbb{Z} \imath \mathbb{Z}$. Therefore, we do not know if these groups admit a $\Phi$-finite confluent rewriting system:

Question 5.23 Is there an invariantly finitely L-presented group that admits a $\Phi$-finite confluent rewriting system but that is not finitely presented? In particular, do the testbed groups in [6] or [9] admit $\Phi$-finite rewriting systems? 
$\overbrace{\text { Appendix }}$

\title{
Investigating self-similar groups using their finite $L$-presentation
}

\begin{abstract}
Self-similar groups provide a rich source of groups with interesting properties; e.g., infinite torsion groups (Burnside groups) and groups with an intermediate word growth. Various self-similar groups can be described by a recursive (possibly infinite) presentation, a so-called finite $L$-presentation. Finite $L$-presentations allow numerous algorithms for finitely presented groups to be generalized to this special class of recursive presentations. We give an overview of the algorithms for finitely $L$-presented groups. As applications, we demonstrate how their implementation in a computer algebra system allows us to study explicit examples of self-similar groups including the FabrykowskiGupta groups. Our experiments yield detailed insight into the structure of these groups.
\end{abstract}

Keywords. Recursive presentations; self-similar groups; Grigorchuk group; Fabrykowski-Gupta groups; coset enumeration; finite index subgroups; Reidemeister-Schreier theorem; nilpotent quotients; solvable quotients.

\section{A.1 Introduction}

The general Burnside problem is among the most influential problems in combinatorial group theory. It asks whether a finitely generated group is finite if every element has finite order. The general Burnside problem was answered negatively by Golod [51]. The first explicit counter-examples were constructed in [5,53,62]. Among these counter-examples is the Grigorchuk group $\mathfrak{G}$ which is a finitely generated self-similar group. The group $\mathfrak{G}$ is not finitely presented [56] but it admits a recursive presentation which could be described in finite terms using the action of a finitely generated monoid of substitutions acting on finitely many relations [96. These recursive presentations are nowadays known 
as finite L-presentations [56] (or endomorphic presentations [6]) in honor of Lysënok's work in [96] for the Grigorchuk group; see [6] or Section A.2 for a definition.

Finite $L$-presentations allow computer algorithms to be employed in the investigation of the groups they define. A first algorithm for finitely $L$-presented groups is the nilpotent quotient algorithm [9,64. Recently, further algorithms for finitely $L$-presented groups were developed [66, 67, 60]. For instance, in [67], a coset enumeration process for finitely $L$-presented groups was described. This is an algorithm which, given a finite generating set of a subgroup of a finitely $L$-presented group, computes the index of the subgroup in the finitely $L$-presented group provided that this index is finite. Usually index computations in self-similar groups have involved lots of tedious calculations (e.g., finding an appropriate quotient of the self-similar group; computing the index of the subgroup in this quotient; followed by a proof that the obtained index is correct; see, for instance, [11, Section 4] or [38, Chapter VIII]). The coset enumerator in [67] makes this process completely automatic and thus it shows the significance of finite $L$-presentations in the investigation of self-similar groups. Moreover, coset enumeration allows one to compute the number of low-index subgroups of finitely $L$-presented groups [67.

We demonstrate the application of the algorithms for finitely $L$-presented groups in the investigation of a class of self-similar groups $\Gamma_{p}$ for $3 \leq p \leq 11$. The group $\Gamma_{3}$ was introduced in [45]. It is a self-similar group with an intermediate word growth [13,45,46]. The groups $\Gamma_{p}$, with $p>3$, were introduced in [57]. They are known as FabrykowskiGupta groups. Their abelianization $\Gamma_{p} / \Gamma_{p}^{\prime} \cong \mathbb{Z}_{p} \times \mathbb{Z}_{p}$ was computed in [57]. Moreover, for $p \geq 5$, the groups $\Gamma_{p}$ are just-infinite, regular branch groups [57]. The congruence subgroups of $\Gamma_{p}$, for primes $p>3$, were studied in [131; see also [49]. The lower central series sections $\gamma_{c} \Gamma_{3} / \gamma_{c+1} \Gamma_{3}$ have been computed entirely in [7] while, for $p>3$, parts of the lower central series sections $\gamma_{c} \Gamma_{p} / \gamma_{c+1} \Gamma_{p}$ have been computed in [9]. So far, little more is known on the groups $\Gamma_{p}$.

For $p \geq 3$, the Fabrykowski-Gupta group $\Gamma_{p}$ admits a finite $L$-presentation [9]. We demonstrate how the implementations of the algorithms for finitely $L$-presented groups allow us to investigate the groups $\Gamma_{p}$ for $3 \leq p \leq 11$ in detail. For instance, we demonstrate the application of our algorithm

- to compute the isomorphism type of the lower central series sections $\gamma_{c} \Gamma_{p} / \gamma_{c+1} \Gamma_{p}$ using improved (parallel) methods from [9,64].

- to compute the isomorphism type of the Dwyer quotients $M_{c}\left(\Gamma_{p}\right)$ of their Schur multiplier using the methods from [66].

- to determine the number of low-index subgroups of the groups $\Gamma_{p}$ using the methods from [67].

- to compute the isomorphism type of the sections $\Gamma_{p}^{(c)} / \Gamma_{p}^{(c+1)}$ of the derived series combining the methods from [70] and $[9,64$ ].

We briefly sketch the algorithms available for finitely $L$-presented groups. Moreover, we compare our experimental results for the Fabrykowski-Gupta groups $\Gamma_{p}$ with those results for the Grigorchuk group $\mathfrak{G}$. The group $\mathfrak{G}$ has been investigated for decades now. 
Even though a lot is known about its structure, various questions still remain open [58]. For further details on the Grigorchuk group $\mathfrak{G}$, we refer to [38, Chapter VIII].

\section{A.2 Self-Similar Groups}

A self-similar group can be defined by its recursive action on a regular rooted tree: Consider the $d$-regular rooted infinite tree $\mathcal{T}_{d}$ as a free monoid over the alphabet $\mathcal{X}=$ $\{0, \ldots, d-1\}$. Then a self-similar group can be defined as follows:

Definition 1.1 A group $G$ acting faithfully on the free monoid $\mathcal{X}^{*}$ is self-similar if for each $g \in G$ and $x \in \mathcal{X}$ there exist $h \in G$ and $y \in \mathcal{X}$ so that

$$
(x w)^{g}=y w^{h} \quad \text { for each } \quad w \in \mathcal{X}^{*} .
$$

It suffices to specify the self-similar action in Eq. A.1) on a generating set of a group. For instance, the Grigorchuk group $\mathfrak{G}=\langle a, b, c, d\rangle$ can be defined as a subgroup of the automorphism group of the rooted binary tree $\mathcal{T}_{2}=\{0,1\}^{*}$ by its self-similar action:

$$
\begin{aligned}
(0 w)^{a} & =1 w & (1 w)^{a} & =0 w \\
(0 w)^{b} & =0 w^{a} & (1 w)^{b} & =1 w^{c} \\
(0 w)^{c} & =0 w^{a} & (1 w)^{c} & =1 w^{d} \\
(0 w)^{d} & =0 w & (1 w)^{d} & =1 w^{b} .
\end{aligned}
$$

The Fabrykowski-Gupta group $\Gamma_{3}$ is another example of a self-similar group. It was introduced in [45] as a group with an intermediate word growth [13,46]. The group $\Gamma_{3}$ was generalized in [57] to a class of self-similar groups $\Gamma_{d}$ acting on the $d$-regular rooted tree:

Definition 1.2 For $d \geq 3$, the Fabrykowski-Gupta group $\Gamma_{d}=\langle a, r\rangle$ is a self-similar group acting faithfully on the d-regular rooted tree $\mathcal{T}_{d}=\{0, \ldots, d-1\}^{*}$ by

$$
\begin{aligned}
& (x w)^{a}=x+1 \quad(\bmod d) w, \text { for } 0 \leq x \leq d-1 \\
& (0 w)^{r}=0 w^{a}, \\
& (x w)^{r}=x w, \quad \text { for } 1 \leq x<d-1 \\
& (d-1 w)^{r}=d-1 w^{r} .
\end{aligned}
$$

The groups $\mathfrak{G}$ and $\Gamma_{d}$ admit a finite $L$-presentation; that is, a finite L-presentation is a group presentation of the form

$$
\left\langle\mathcal{X} \mid \mathcal{Q} \cup \bigcup_{\sigma \in \Phi^{*}} \mathcal{R}^{\sigma}\right\rangle
$$

where $\mathcal{X}$ is a finite alphabet, $\mathcal{Q}$ and $\mathcal{R}$ are finite subsets of the free group $F$ over $\mathcal{X}$, and $\Phi^{*}$ denotes the monoid of endomorphisms which is generated by the finite set $\Phi \subseteq$ $\operatorname{End}(F)$. The group defined by the finite $L$-presentation in Eq. (A.2) is denoted by $\langle\mathcal{X}|\mathcal{Q}| \Phi \mid \mathcal{R}\rangle$. If $\mathcal{Q}=\emptyset$ holds, the $L$-presentation in Eq. (A.2) is ascending. In this case, every endomorphism $\sigma \in \Phi^{*}$ induces an endomorphism of the group $G$. 
The Grigorchuk group $\mathfrak{G}$ is an example of a self-similar group which is finitely $L$ presented [96]: the group $\mathfrak{G}$ satisfies

$$
\left.\mathfrak{G} \cong\langle\{a, b, c, d\}|\left\{a^{2}, b^{2}, c^{2}, d^{2}, b c d\right\} \cup \bigcup_{i \geq 0}\left\{(a d)^{4},(\text { adacac })^{4}\right\}^{\sigma^{i}}\right\rangle,
$$

where $\sigma$ is the endomorphism of the free group $F$ over $\{a, b, c, d\}$ which is induced by the map $a \mapsto a c a, b \mapsto d, c \mapsto b$, and $d \mapsto c$. A general method for computing a finite $L$-presentation for a class of self-similar groups was developed in [6] in order to prove

Theorem 1.3 (Bartholdi [6]) Each finitely generated, contracting, semi-fractal regular branch group is finitely L-presented; however, it is not finitely presented.

The constructive proof of Theorem [1.3] in [6] was used in [9] to compute the following finite $L$-presentation for the Fabrykowski-Gupta group $\Gamma_{p}$ :

Theorem 1.4 (Bartholdi et al. 99) For $d \geq 3$, the group $\Gamma_{d}$ is finitely L-presented by $\langle\{\alpha, \rho\}|\emptyset|\{\varphi\} \mid \mathcal{R}\rangle$ where the iterated relations in $\mathcal{R}$ are defined as follows: Writing $\sigma_{i}=\rho^{\alpha^{i}}$, for $1 \leq i \leq d-1$, and reading indices modulo $d$, we have

$$
\mathcal{R}=\left\{\alpha^{d},\left[\sigma_{i}^{\sigma_{i-1}^{k}}, \sigma_{j}^{\sigma_{j-1}^{\ell}}\right], \sigma_{i}^{-\sigma_{i-1}^{k+1}} \sigma_{i}^{\sigma_{i-1}^{k} \sigma_{i-1}^{\sigma_{i-2}^{\ell}}}\right\}_{1 \leq i, j \leq d, 2 \leq|i-j| \leq d-2,0 \leq k, \ell \leq d-1}
$$

The substitution $\varphi$ is induced by the map $\alpha \mapsto \rho^{\alpha^{-1}}$ and $\rho \mapsto \rho$.

It follows immediately from the $L$-presentation in Theorem 1.4 that the substitution $\varphi$ induces an endomorphism of the group $\Gamma_{d}$. Finite $L$-presentations $\langle\mathcal{X}|\mathcal{Q}| \Phi \mid \mathcal{R}\rangle$ whose substitutions $\sigma \in \Phi$ induce endomorphisms of the group are invariant L-presentations. Each ascending $L$-presentation is invariant. It is also easy to see that the $L$-presentation for the Grigorchuk group $\mathfrak{G}$ above is invariant [55, Corollary 4].

A finite $L$-presentation allows us to define a group that is possibly infinitely presented in computer algebra systems such as GAP [50] or Magma [28]. Beside defining a selfsimilar group by its finite $L$-presentation, it can also be defined by its recursive action on a regular tree. A finite approximation of the recursive action of a self-similar group is often sufficient to study finite index subgroups since various self-similar groups have the congruence property: every finite index subgroup contains a level stabilizer (i.e., the stabilizer of some level of the regular tree). This often yields an alternative approach to investigate the structure of a self-similar group with the help of computer algebra systems [8]. However, there are self-similar groups that do not have the congruence property [14]. For these groups, their finite $L$-presentation may help to gain insight into the structure of the group. The groups $\mathfrak{G}$ and $\Gamma_{3}$ have the congruence property [11].

In the following, we demonstrate how the finite $L$-presentation in Theorem 1.4 allows us to obtain detailed information on the structure of the groups $\Gamma_{p}$, for $3 \leq p \leq 11$. For further details on self-similar groups, we refer to the monograph by Nekrashevych [101]. 


\section{A.3 A Nilpotent Quotient Algorithm}

For a group $G$, the lower central series is defined recursively by $\gamma_{1} G=G$ and $\gamma_{c+1}=$ $\left[\gamma_{c} G, G\right]$ for $c \in \mathbb{N}$. If $G$ is finitely generated, $G / \gamma_{c+1} G$ is polycyclic and therefore it can be described by a polycyclic presentation; i.e., a polycyclic presentation is a finite presentation whose generators refine a subnormal series with cyclic sections. A polycyclic presentation allows effective computations within the group it defines [129, Chapter 9].

A nilpotent quotient algorithm computes a polycyclic presentation for the factor group $G / \gamma_{c+1} G$ together with a homomorphism $G \rightarrow G / \gamma_{c+1} G$. Such an algorithm for finitely presented groups was developed in [107]. This nilpotent quotient algorithm was a first algorithm that could be generalized to finite $L$-presentations [9, 64]. The experimental results in this section were obtained with an improved, parallel version of the algorithm in [9,64]. They extend the computational results in [9] significantly.

We briefly sketch the nilpotent quotient algorithm for finitely $L$-presented groups in the following. Let $G=\langle\mathcal{X}|\mathcal{Q}| \Phi \mid \mathcal{R}\rangle$ be a finitely $L$-presented group. Denote by $F$ the free group over the alphabet $\mathcal{X}$ and let $K$ be the normal closure $K=\left\langle\bigcup_{\sigma \in \Phi^{*}} \mathcal{R}^{\sigma}\right\rangle^{F}$. First, we assume that $\mathcal{Q}=\emptyset$ holds. Then $K^{\sigma} \subseteq K$, for each $\sigma \in \Phi$, and $G=F / K$ hold. Therefore, each $\sigma \in \Phi$ induces an endomorphism of the group $G$. Furthermore, we have $G / \gamma_{c} G \cong F / K \gamma_{c} F$. The nilpotent quotient algorithm uses an induction on $c$ to compute a polycyclic presentation for $G / \gamma_{c} G$. For $c=2$, we have

$$
G /[G, G] \cong F / K F^{\prime} \cong\left(F / F^{\prime}\right) /\left(K F^{\prime} / F^{\prime}\right) .
$$

Since $G$ is finitely generated, $F / F^{\prime}$ is free abelian with finite rank. The normal generators $\bigcup_{\sigma \in \Phi^{*}} \mathcal{R}^{\sigma}$ of $K$ give a (possibly infinite) generating set of $K F^{\prime} / F^{\prime}$. From this generating set it is possible to compute a finite generating set $\mathcal{U}$ with a spinning algorithm. The finite generating set $\mathcal{U}$ allows us to apply the methods from [107] that eventually compute a polycyclic presentation for $F / K F^{\prime}$ together with a homomorphism $F \rightarrow F / K F^{\prime}$ which induces $G \rightarrow G / G^{\prime}$.

For $c>2$, assume that the algorithm has already computed a polycyclic presentation for $G / \gamma_{c} G \cong F / K \gamma_{c} F$ together with a homomorphism $F \rightarrow F / K \gamma_{c} F$. Consider the factor group $H_{c+1}=F /\left[K \gamma_{c} F, F\right]$. Then $\left[K \gamma_{c} F, F\right]=[K, F] \gamma_{c+1} F$ and $H_{c+1}$ satisfies the short exact sequence

$$
1 \rightarrow K \gamma_{c} F /\left[K \gamma_{c} F, F\right] \rightarrow H_{c+1} \rightarrow F / K \gamma_{c} F \rightarrow 1
$$

that is, $H_{c+1}$ is a central extension of a finitely generated abelian group by $G / \gamma_{c} G$. Thus $H_{c+1}$ is nilpotent and polycyclic. A polycyclic presentation for $H_{c+1}$ together with a homomorphism $F \rightarrow F /\left[K \gamma_{c} F, F\right]$ can be computed with the covering algorithm in [107]; for a proof that this algorithm generalizes to finite $L$-presentations we refer to 64. Then $K \gamma_{c+1} F /\left[K \gamma_{c}, F\right]$ is a subgroup of $K \gamma_{c} F /\left[K \gamma_{c} F, F\right]$ and a (possibly infinite) generating set for $K \gamma_{c+1} F /\left[K \gamma_{c} F, F\right]$ can be obtained from the normal generators of $K$. Again, a finite generating set $\mathcal{U}$ for $K \gamma_{c+1} F /\left[K \gamma_{c} F, F\right]$ can be computed with a spinning algorithm from the normal generators of $K$. The finite generating set $\mathcal{U}$ allows us to apply the 
methods in 107 for computing a polycyclic presentation for $G / \gamma_{c+1} G \cong F / K \gamma_{c+1} F$ together with a homomorphism $F \rightarrow F / K \gamma_{c+1} F$. This finishes our description of the nilpotent quotient algorithm in the case where $Q=\emptyset$ holds.

If, on the other hand, $G$ is given by a finite $L$-presentation $\langle\mathcal{X}|\mathcal{Q}| \Phi \mid \mathcal{R}\rangle$ with $\mathcal{Q} \neq \emptyset$, the algorithm described above applies to the finitely $L$-presented group $H=\langle\mathcal{X}|$ $\emptyset|\Phi| \mathcal{R}\rangle$. Write $H=F / K$ and $G=F / L$ for normal subgroups $K \leq L$. The nilpotent quotient algorithm applied to $H$ yields a polycyclic presentation for $H / \gamma_{c+1} H$ together with a homomorphism $F \rightarrow F / K \gamma_{c+1} F$. This yields

$$
G / \gamma_{c+1} G \cong F / L \gamma_{c+1} F \cong\left(F / K \gamma_{c+1} F\right) /\left(L \gamma_{c+1} F / K \gamma_{c+1} F\right)
$$

The subgroup $L \gamma_{c+1} F / K \gamma_{c+1} F$ is finitely generated by the images of the relations in $\mathcal{Q}$. Standard methods for polycyclic groups 129 then give a polycyclic presentation for the factor group $G / \gamma_{c+1} G$ of the polycyclically presented group $H / \gamma_{c+1} H$ and a homomorphism $F \rightarrow G / \gamma_{c+1} G$.

\section{A.3.1 Applications of the Nilpotent Quotient Algorithm}

The nilpotent quotient algorithm allows us to compute within the lower central series quotients $G / \gamma_{c+1} G$ of a finitely $L$-presented group $G$. For instance, it allows us to determine the isomorphism type of the lower central series sections $\gamma_{c} G / \gamma_{c+1} G$. For various self-similar groups, the lower central series sections often exhibit periodicities. For instance, the Grigorchuk group $\mathfrak{G}$ satisfies

Theorem 1.5 (Rozhkov [123]) The lower central series sections $\gamma_{c} \mathfrak{G} / \gamma_{c+1} \mathfrak{G}$ are 2 elementary abelian with the following 2-ranks:

$$
\operatorname{rk}_{2}\left(\gamma_{c} \mathfrak{G} / \gamma_{c+1} \mathfrak{G}\right)=\left\{\begin{array}{cl}
3 \text { or } 2, & \text { if } c=1 \text { or } c=2, \text { respectively } \\
2, & \text { if } c \in\left\{2 \cdot 2^{m}+1, \ldots, 3 \cdot 2^{m}\right\} \\
1, & \text { if } c \in\left\{3 \cdot 2^{m}+1, \ldots, 4 \cdot 2^{m}\right\}
\end{array}\right\} \text { with } m \in \mathbb{N}_{0} \text {. }
$$

The group $\mathfrak{G}$ has finite width 2 .

Our implementation of the nilpotent quotient algorithm in 65] allows a computer algebra system to be applied in the investigation of the quotients $G / \gamma_{c} G$ for a finitely $L$-presented group $G$. For instance, our implementation suggests that the group $\Gamma_{d}$ has a maximal nilpotent quotient whenever $d$ is not a prime-power. Based on this experimental observation, the following proposition was proved:

Proposition 1.6 (Bartholdi et al. [9]) If $d$ is not a prime-power, the group $\Gamma_{d}$ has a maximal nilpotent quotient. Its nilpotent quotients are isomorphic to the nilpotent quotients of the wreath product $\mathbb{Z}_{d} \curlywedge \mathbb{Z}_{d}$.

For a prime $p \geq 3$, the lower central series sections $\gamma_{c} \Gamma_{p} / \gamma_{c+1} \Gamma_{p}$ are $p$-elementary abelian. For $p=3$, the lower central series sections $\gamma_{c} \Gamma_{3} / \gamma_{c+1} \Gamma_{3}$ were computed in [7: 
Proposition 1.7 (Bartholdi [7]) The sections $\gamma_{c} \Gamma_{3} / \gamma_{c+1} \Gamma_{3}$ are 3-elementary abelian with the following 3-ranks:

$$
\operatorname{rk}_{3}\left(\gamma_{c} \Gamma_{3} / \gamma_{c+1} \Gamma_{3}\right)=\left\{\begin{array}{cl}
2 \text { or } 1, & \text { if } c=1 \text { or } c=2, \text { respectively, } \\
2, & \text { if } c \in\left\{3^{k}+2, \ldots, 2 \cdot 3^{k}+1\right\}, \\
1, & \text { if } c \in\left\{2 \cdot 3^{k}+2, \ldots, 3^{k+1}+1\right\}
\end{array}\right\}
$$

with $k \in \mathbb{N}_{0}$. The group $\Gamma_{3}$ has finite width 2 .

For primes $p>3$, little is known about the series sections $\gamma_{c} \Gamma_{p} / \gamma_{c+1} \Gamma_{p}$ so far [9]. We use the following abbreviation to list the ranks of these sections: If the same entry $a \in \mathbb{N}$ appears in $m$ consecutive places in a list, it is listed once in the form $a^{[m]}$. The sections $\gamma_{c} \Gamma_{p} / \gamma_{c+1} \Gamma_{p}$ are $p$-elementary abelian. Their $p$-ranks are given by the following table:

\begin{tabular}{clc}
\hline$p$ & \multicolumn{1}{c}{$\operatorname{rk}_{p}\left(\gamma_{c} \Gamma_{p} / \gamma_{c+1} \Gamma_{p}\right)$} & class \\
\hline 3 & $2,1^{[1]}, 2^{[1]}, 1^{[1]}, 2^{[3]}, 1^{[3]}, 2^{[9]}, 1^{[9]}, 2^{[27]}, 1^{[27]}, 2^{[65]}$ & 147 \\
5 & $2,1^{[3]}, 2^{[1]}, 1^{[13]}, 2^{[5]}, 1^{[65]}, 2^{[25]}, 1^{[26]}$ & 139 \\
7 & $2,1^{[5]}, 2^{[1]}, 1^{[33]}, 2^{[7]}, 1^{[68]}$ & 115 \\
11 & $2,1^{[9]}, 2^{[1]}, 1^{[97]} 2^{[4]}$ & 112 \\
\hline
\end{tabular}

These computational results were obtained with a parallel version of the nilpotent quotient algorithm in [9, 64]. They were intended to be published in [42]. These computational results extend those in [9] significantly so that we obtain detailed conjectures on the structure of the lower central series sections $\gamma_{c} \Gamma_{p} / \gamma_{c+1} \Gamma_{p}$ : The sections $\gamma_{c} \Gamma_{p} / \gamma_{c+1} \Gamma_{p}$ are $p$-elementary abelian with the following $p$-ranks: Write $f_{p}(\ell)=p+\left(p^{2}-2 p-1\right)\left(p^{\ell+1}-\right.$ $1) /(p-1)$ and $g_{p}(\ell)=f_{p}(\ell)+p^{\ell+1}$. Then we conjecture that

$$
\operatorname{rk}_{p}\left(\gamma_{c} \Gamma_{p} / \gamma_{c+1} \Gamma_{p}\right)= \begin{cases}2, & \text { if } c \in\{1, p\} \text { or } f_{p}(\ell) \leq c<g_{p}(\ell) \text { for some } \ell \in \mathbb{N}_{0}, \\ 1, & \text { otherwise }\end{cases}
$$

holds. If this conjecture is true, the group $\Gamma_{p}$ would have finite width 2 . For prime powers $3 \leq d \leq 11$, our implementation yields the following results:

- For $d=4$, the Fabrykowski-Gupta group $\Gamma_{4}$ satisfies

$$
\Gamma_{4} / \Gamma_{4}^{\prime} \cong \mathbb{Z}_{4} \times \mathbb{Z}_{4} \text { and } \gamma_{2} \Gamma_{4} / \gamma_{3} \Gamma_{4} \cong \mathbb{Z}_{4}
$$

For $3 \leq c \leq 141$, the sections $\gamma_{c} \Gamma_{4} / \gamma_{c+1} \Gamma_{4}$ are 2-elementary abelian with 2-ranks: $2^{[4]}, 3^{[3]}, 2^{[13]}, 3^{[12]}, 2^{[52]}, 3^{[48]}, 2^{[7]}$.

- For $d=8$, the Fabrykowski-Gupta group $\Gamma_{8}$ satisfies

$$
\Gamma_{8} / \Gamma_{8}^{\prime} \cong \mathbb{Z}_{8} \times \mathbb{Z}_{8}, \quad \gamma_{2} \Gamma_{8} / \gamma_{3} \Gamma_{8} \cong \mathbb{Z}_{8}
$$

and

$$
\gamma_{3} \Gamma_{8} / \gamma_{4} \Gamma_{8} \cong \gamma_{4} \Gamma_{8} / \gamma_{5} \Gamma_{8} \cong \gamma_{5} \Gamma_{8} / \gamma_{6} \Gamma_{8} \cong \gamma_{6} \Gamma_{8} / \gamma_{7} \Gamma_{8} \cong \mathbb{Z}_{4}
$$

For $7 \leq c \leq 111$, the sections $\gamma_{c} \Gamma_{8} / \gamma_{c+1} \Gamma_{8}$ are 2-elementary abelian with 2-ranks: $2,1,2^{[2]}, 3,2,3^{[2]}, 4,3^{[8]}, 2^{[23]}, 3^{[5]}, 2^{[3]}, 1^{[8]}, 2^{[16]}, 3^{[8]}, 2^{[8]}, 3^{[16]}, 4$. 
- For $d=9$, the Fabrykowski-Gupta group $\Gamma_{9}$ satisfies

$$
\Gamma_{9} / \Gamma_{9}^{\prime} \cong \mathbb{Z}_{9} \times \mathbb{Z}_{9}, \quad \gamma_{2} \Gamma_{9} / \gamma_{3} \Gamma_{9} \cong \mathbb{Z}_{9}, \quad \text { and } \quad \gamma_{3} \Gamma_{9} / \gamma_{4} \Gamma_{9} \cong \mathbb{Z}_{9}
$$

For $4 \leq c \leq 117$, the sections $\gamma_{c} \Gamma_{9} / \gamma_{c+1} \Gamma_{9}$ are 3 -elementary abelian with 3-ranks: $1^{[5]}, 2^{[6]}, 3,2^{[17]}, 1^{[38]}, 1^{[47]}$.

\section{A.4 Computing Dwyer Quotients of the Schur Multiplier}

The Schur multiplier $M(G)$ of a group $G$ can be defined as the second homology group $H_{2}(G, \mathbb{Z})$ with integer coefficients. It is an invariant of the group which is of particular interest for infinitely presented groups because proving the Schur multiplier being infinitely generated proves that the group does not admit a finite presentation. This is due to the fact that the Schur multiplier of a finitely presented group is finitely generated abelian which can be seen as a consequence of Hopf's formula: If $F$ is a free group and $R \unlhd F$ a normal subgroup so that $G \cong F / R$ holds, the Schur multiplier $M(G)$ satisfies

$$
M(G) \cong\left(R \cap F^{\prime}\right) /[R, F] .
$$

However, a group with a finitely generated Schur multiplier is not necessarily finitely presented [18. For further details on the Schur multiplier, we refer to [121, Chapter 11].

It is known that the Schur multiplier of a finitely $L$-presented group (and even the Schur multiplier of a finitely presented group) is not computable in general [52]. Nevertheless, the Schur multiplier of some self-similar groups has been computed in [14, 56]: For instance, the Grigorchuk group $\mathfrak{G}$ satisfies

Proposition 1.8 (Grigorchuk [56]) The Schur multiplier $M(\mathfrak{G})$ is infinitely generated 2-elementary abelian. Therefore, the group $\mathfrak{G}$ is not finitely presented.

There are various examples of self-similar groups for which nothing is known on their Schur multiplier. Even though the Schur multiplier $M(G)$ is not computable in general, it is possible to compute successive quotients of $M(G)$ provided that the group $G$ is given by an invariant finite $L$-presentation [66]. These quotients often exhibit periodicities as well: For instance, our experiments with the implementation of the algorithm in 66 suggest that the Schur multiplier of the Fabrykowski-Gupta groups $\Gamma_{d}$, for a primepower $d=p^{\ell}$, is infinitely generated. The algorithm for computing successive quotients of $M(G)$ provides a first method to investigate the structure of the Schur multiplier of an invariantly finitely $L$-presented group (and even the Schur multiplier of a finitely presented group).

We briefly sketch the idea of this algorithm: Let $G$ be an invariantly finitely $L$ presented group. Write $G \cong F / K$ for a free group $F$ and a normal subgroup $K$. Then $G / \gamma_{c} G \cong F / K \gamma_{c} F$. We identify $M(G)$ with $\left(K \cap F^{\prime}\right) /[K, F]$ and $M\left(G / \gamma_{c} G\right)$ with $\left(K \gamma_{c} F \cap F^{\prime}\right) /\left[K \gamma_{c} F, F\right]$ and define

$$
\varphi_{c}: M(G) \rightarrow M\left(G / \gamma_{c} G\right), g[K, F] \mapsto g\left[K \gamma_{c} F, F\right] .
$$


Then $\varphi_{c}$ is a homomorphism of abelian groups. In the induction step of the nilpotent quotient algorithm, the algorithm computes a homomorphism $F \rightarrow F /\left[K \gamma_{c} F, F\right]$. This homomorphism allows us to compute the image of the Schur multiplier $M(G)$ in $M\left(G / \gamma_{c} G\right)$. In particular, it allows us to compute the isomorphism type of the Dwyer quotient $M_{c}(G)=M(G) / \operatorname{ker} \varphi_{c}$, for $c \in \mathbb{N}$, where

$$
M(G) \geq \operatorname{ker} \varphi_{1} \geq \operatorname{ker} \varphi_{2} \geq \ldots
$$

The algorithm for computing $M_{c}(G)$ has been implemented in GAP. Its implementation allows us to compute the Dwyer quotients of various self-similar groups: Since the Schur multiplier of the Grigorchuk group $\mathfrak{G}$ is 2-elementary abelian, the Dwyer quotients of $\mathfrak{G}$ are 2-elementary abelian. We have computed the Dwyer quotients $M_{c}(\mathfrak{G})$ for $1 \leq c \leq 301$. These quotients are 2-elementary abelian with the following 2-ranks:

$$
1,2,3^{[3]}, 5^{[6]}, 7^{[12]}, 9^{[24]}, 11^{[48]}, 13^{[96]}, 15^{[110]} \text {. }
$$

These experiments suggest that the Grigorchuk group satisfies

$$
M_{c}(\mathfrak{G}) \cong\left\{\begin{array}{cl}
\mathbb{Z}_{2} \text { or }\left(\mathbb{Z}_{2}\right)^{2}, & \text { if } c=1 \text { or } c=2, \text { respectively, } \\
\left(\mathbb{Z}_{2}\right)^{2 m+3}, & \text { if } c \in\left\{3 \cdot 2^{m}, \ldots, 3 \cdot 2^{m+1}-1\right\}
\end{array}\right\}
$$

with $m \in \mathbb{N}_{0}$. For the Fabrykowski-Gupta groups $\Gamma_{d}$, the algorithm in [66] yields first insight into the structure of $M\left(\Gamma_{d}\right)$ : We restrict ourself to the groups $\Gamma_{d}$ for prime powers $d=p^{\ell}$ because, otherwise, the groups have a maximal nilpotent quotient by Proposi-

\begin{tabular}{|c|c|}
\hline$p$ & $\operatorname{rk}_{p}\left(M_{c}\left(\Gamma_{p}\right)\right)$ \\
\hline 3 & $0^{[2]}, 1^{[3]}, 2^{[0]}, 3^{[9]}, 4^{[1]}, 5^{[26]}, 6^{[4]}, 7^{[77]}, 8^{[13]}, 9^{[12]}$ \\
\hline 5 & $0^{[1]}, 1^{[4]}, 2^{[2]}, 3^{[20]}, 4^{[10]}, 5^{[100]}, 6^{[1]}$ \\
\hline 7 & $0^{[1]}, 1^{[2]}, 2^{[6]}, 3^{[2]}, 4^{[14]}, 5^{[42]}, 6^{[14]}, 7^{[34]}$ \\
\hline 11 & $0^{[1]}, 1^{[2]}, 2^{[2]}, 3^{[2]}, 4^{[10]}, 5^{[2]}, 6^{[22]}, 7^{[22]}, 8^{[22]}, 9^{[27]}$ \\
\hline
\end{tabular}
tion [1.6. For a prime $p \in\{3,5,7,11\}$, the Dwyer quotients $M_{c}\left(\Gamma_{p}\right)$ are $p$-elementary abelian groups with the following $p$-ranks:

As noted by Bartholdi, these experimental results suggest that

$$
\operatorname{rk}_{3}\left(M_{c+1}\left(\Gamma_{3}\right)\right)=\left\{\begin{array}{cl}
2\left\lfloor\log _{3}\left(\frac{2 c-1}{10}\right)\right\rfloor+3, & \text { if } \log _{3}(2 c-1) \in \mathbb{Z}, \\
\left\lfloor\log _{3}(2 c-1)\right\rfloor+\left\lfloor\log _{3}\left(\frac{2 c-1}{10}\right)\right\rfloor+1, & \text { otherwise }
\end{array}\right.
$$

for $c \geq 6$. Our results for the Dwyer quotients $M_{c}\left(\Gamma_{d}\right)$, for $d \in\{4,8,9\}$, are shown in Table A.4 where we list the abelian invariants of $M_{c}(G)$. Here, a list $\left(\alpha_{1}, \ldots, \alpha_{n}\right)$ stands for the abelian group $\mathbb{Z}_{\alpha_{1}} \times \cdots \times \mathbb{Z}_{\alpha_{n}}$. Again, we list the abelian invariants $\left(\alpha_{1}, \ldots, \alpha_{n}\right)^{[m]}$ just once if they appear in $m$ consecutive places. 
Table A.1: Dwyer quotients of the Fabrykowski-Gupta groups $\Gamma_{d}$

\begin{tabular}{|c|c|}
\hline$d$ & $M_{c}\left(\Gamma_{d}\right)$ \\
\hline 4 & $\begin{array}{r}(1)^{[1]}(2)^{[1]}(2,2)^{[1]}(2,4)^{[4]}(2,2,2,4)^{[1]} \\
(2,2,2,2,4)^{[4]}(2,2,2,4,4)^{[16]}(2,2,2,2,4,4)^{[1]}(2,2,2,2,2,4,4)^{[3]} \\
(2,2,2,2,2,2,4,4)^{[16]}(2,2,2,2,2,4,4,4)^{[64]}(2,2,2,2,2,2,4,4,4)^{[5]} \\
(2,2,2,2,2,2,2,4,4,4)^{[11]}(2,2,2,2,2,2,2,2,4,4,4)^{[26]}\end{array}$ \\
\hline 8 & $\begin{array}{r}(1)^{[1]}(8)^{[2]}(4,8)^{[3]}(2,4,8)^{[4]}(2,8,8)^{[1]}(2,2,8,8)^{[2]} \\
(2,2,2,8,8)^{[2]}(2,2,4,8,8)^{[2]}(2,4,4,8,8)^{[2]}(2,4,8,8,8)^{[2]} \\
(2,8,8,8,8)^{[8]}(2,2,8,8,8,8)^{[4]}(2,4,8,8,8,8)^{[20]}(2,2,4,8,8,8,8)^{[32]} \\
(2,2,8,8,8,8,8)^{[7]}(2,2,2,8,8,8,8,8)^{[16]}(2,2,2,2,8,8,8,8,8)^{[16]} \\
(2,2,2,4,8,8,8,8,8)^{[16]}(2,2,4,4,8,8,8,8,8)^{[3]}\end{array}$ \\
\hline 9 & $\begin{array}{r}(1)^{[1]}(9)^{[2]}(3,9)^{[2]}(3,3,9)^{[4]}(3,9,9)^{[2]} \\
(9,9,9)^{[2]}(3,9,9,9)^{[2]}(3,3,9,9,9)^{[4]}(3,9,9,9,9)^{[2]} \\
(9,9,9,9,9)^{[12]}(3,9,9,9,9,9)^{[18]}(3,3,9,9,9,9,9)^{[36]} \\
(3,9,9,9,9,9,9)^{[18]}(9,9,9,9,9,9,9)^{[17]}(3,9,9,9,9,9,9,9)^{[12]}\end{array}$ \\
\hline
\end{tabular}

\section{A.5 Coset Enumeration for Finite Index Subgroups}

A standard algorithm for finitely presented groups is the coset enumerator introduced by Todd and Coxeter [133]. Coset enumeration is an algorithm that, given a finite generating set of a subgroup $H \leq G$, computes the index $[G: H]$ provided that this index is finite. Its overall strategy is to compute a permutation representation for the group's action on the right-cosets $H \backslash G$. For finitely presented groups, coset enumeration techniques have been investigate for some time [32, 88, 102, 129. They allow computer algorithms to be applied in the investigation of finitely presented groups by their finite index subgroups [77]. It was shown in [67], that even finitely $L$-presented groups allow one to develop a coset enumeration process. This latter algorithm reduces the computation to finite presentations first and then it proves correctness of the obtained result. A coset enumerator for finitely $L$-presented groups has various interesting applications: For instance, it allows one to compute low-index subgroups, as suggested in [40], and it solves the generalized word problem for finite index subgroups [67.

We briefly sketch the idea of the coset enumeration process in [67] in the following. Let $G=\langle\mathcal{X}|\mathcal{Q}| \Phi \mid \mathcal{R}\rangle$ be a finitely $L$-presented group. Suppose that a subgroup $H \leq G$ is given by its finitely many generators $\left\{g_{1}, \ldots, g_{n}\right\}$. We consider the generators $g_{1}, \ldots, g_{n}$ as elements of the free group $F$ over $\mathcal{X}$. Then $E=\left\langle g_{1}, \ldots, g_{n}\right\rangle \leq F$ satisfies $H \cong E K / K$ where $K=\left\langle\mathcal{Q} \cup \bigcup_{\sigma \in \Phi^{*}} \mathcal{R}^{\sigma}\right\rangle^{F}$ is the kernel of the free presentation. We are to compute the index $[G: H]=[F: E K]$. For this purpose, we define $\Phi_{\ell}=\left\{\sigma \in \Phi^{*} \mid\|\sigma\| \leq \ell\right\}$ where $\|\cdot\|$ denotes the usual word-length in the free monoid $\Phi^{*}$. Consider the finitely 
presented groups $G_{\ell}=F / K_{\ell}$ given by the finite presentation

$$
G_{\ell}=\left\langle\mathcal{X} \mid \mathcal{Q} \cup \bigcup_{\sigma \in \Phi_{\ell}} \mathcal{R}^{\sigma}\right\rangle
$$

Then $G_{\ell}$ naturally maps onto $G$ and we obtain a series of subgroups

$$
E K_{0} \leq E K_{1} \leq \ldots \leq E K \leq F
$$

Since $E K \leq F$ is a finite index subgroup of a finitely generated group, it is finitely generated by $u_{1}, \ldots, u_{n}$, say. Furthermore, we have $E K=\bigcup_{\ell \geq 0} E K_{\ell}$. For each $u_{i} \in E K$, there exists $n_{i} \in \mathbb{N}_{0}$ so that $u_{i} \in E K_{n_{i}}$. For $m=\max \left\{n_{i} \mid 1 \leq i \leq n\right\}$ we have $\left\{u_{1}, \ldots, u_{n}\right\} \subseteq E K_{m}$. Thus $E K=E K_{m}$. In fact, there exists a positive integer $m \in \mathbb{N}_{0}$ so that $H$ has finite index in the finitely presented group $G_{m}=\left\langle\mathcal{X} \mid \mathcal{Q} \cup \bigcup_{\sigma \in \Phi_{m}} \mathcal{R}^{\sigma}\right\rangle$.

Coset enumeration for finitely presented groups allows us to compute a permutation representation $\pi: F \rightarrow \operatorname{Sym}\left(E K_{m} \backslash F\right)$. The integer $m$ cannot be given a priori. However, the following straightforward approach yields an algorithm for computing $\ell \in \mathbb{N}$ so that $\left[F: E K_{\ell}\right]$ is finite: Start with an arbitrary $\ell \in \mathbb{N}$ and run the coset enumerator for finitely presented groups with an upper bound $N$ on the number of intermediate cosets defined in its process. If this coset enumerator does not terminate successfully, we both increase the index $\ell$ and the upper bound $N$. We then run both algorithms in parallel using the increased bound $N^{\prime}$. We continue to increase these numbers and to run all these algorithms in parallel until eventually one of them terminates. Termination of this process is guaranteed for a sufficiently large integer $\ell$ because $[G: H]$ was assumed finite.

Suppose that $\left[G_{\ell}: H\right]$ is finite and that the coset enumerator has computed a permutation representation $\pi_{\ell}: F \rightarrow \operatorname{Sym}\left(E K_{\ell} \backslash F\right)$. Then $[G: H]=[F: E K]$ divides the index $\left[G_{\ell}: H\right]=\left[F: E K_{\ell}\right]$. It suffices to check whether or not $\pi_{\ell}$ induces a group homomorphism $G \rightarrow \operatorname{Sym}\left(E K_{\ell} \backslash F\right)$. In this case, we obtain $\left[G_{\ell}: H\right]=[G: H]$ and $\pi_{\ell}$ is a permutation representation for $G$ 's action on the right-cosets $H \backslash G$. Otherwise, we have to enlarge the index $\ell$ and we would finally compute the index $[G: H]$ in this way. The following theorem was proved in [67]:

Theorem 1.9 For a finitely L-presented group $G=\langle\mathcal{X}|\mathcal{Q}| \Phi \mid \mathcal{R}\rangle$ and a homomorphism $\pi: F \rightarrow H$ into a finite group $H$, there exists an algorithm that decides whether or not $\pi$ induces a group homomorphism $G \rightarrow H$.

Proof. For an explicit algorithm, we refer to [67].

Coset enumeration for finitely $L$-presented groups allows various computations with finite index subgroups; e.g. computing the intersection of two finite index subgroups, computing the core of a finite index subgroup, solving the generalized word problem for finite index subgroups, etc. In the following, we demonstrate the application of our coset enumerator to the Fabrykowski-Gupta groups $\Gamma_{p}$. In particular, we show how to compute the number of finite index subgroups with a moderate index. 


\section{A.5.1 An Application of Coset Enumeration: Low-Index Subgroups}

As an application of the coset enumeration process, we consider subgroups with small index in a finitely $L$-presented group. Since the finitely presented group $G_{\ell}$ from Eq. (A.4) naturally maps onto the finitely $L$-presented group $G$, it suffices to compute low-index subgroups of the finitely presented group $G_{\ell}$. These subgroups map to subgroups of $G$ with possibly smaller index. On the other hand, each finite index subgroup of $G$ has a full preimage with same index in $G_{\ell}$. Therefore it remains to remove duplicates from the list of subgroups obtained from the finitely presented group $G_{\ell}$. For finitely presented groups, an algorithm for computing all subgroups up to a given index was described in [40]. An implementation of this algorithm can be found in [41. This implementation includes an algorithm for computing only the normal subgroups of a finitely presented group [35]. The latter algorithm allows to deal with possibly larger indices than the usual low-index subgroup algorithms.

We first consider the Grigorchuk group $\mathfrak{G}$ : its lattice of normal subgroups is wellunderstood [7, 33] while its lattice of finite index subgroups is widely unknown [58]. It is known that the Grigorchuk group has seven subgroups of index two [58]. In [116], it was shown that these index-two subgroups are the only maximal subgroups of $\mathfrak{G}$. The implementation of our coset enumeration process allows us to compute the number of subgroups with index at most 64 in the group $\mathfrak{G}$ [67]. Our computations correct the counts in [12, Section 7.4] and [11, Section 4.1]. The following list summarizes the number of subgroups $(\leq)$ and the number of normal subgroups $(\unlhd)$ of $\mathfrak{G}$ :

\begin{tabular}{cccccccc}
\hline index & 1 & 2 & 4 & 8 & 16 & 32 & 64 \\
\hline$\leq$ & 1 & 7 & 31 & 183 & 1827 & 22931 & 378403 \\
$\unlhd$ & 1 & 7 & 7 & 7 & 5 & 3 & 3 \\
\hline
\end{tabular}

For the Fabrykowski-Gupta groups $\Gamma_{p}$, where $3 \leq p \leq 11$ is prime, we only found subgroups with prime-power index in $\Gamma_{p}$. Their counts are as follows:

\begin{tabular}{cccccccccc}
\hline \multirow{3}{*}{ index } & \multicolumn{3}{c}{$p=3$} & \multicolumn{3}{c}{$p=5$} & \multicolumn{2}{c}{$p=7$} & \multicolumn{2}{c}{$p=11$} \\
& $\leq$ & $\unlhd$ & $\leq$ & $\unlhd$ & $\leq$ & $\unlhd$ & $\leq$ & $\unlhd$ \\
\hline$p^{0}$ & 1 & 1 & 1 & 1 & 1 & 1 & 1 & 1 \\
$p^{1}$ & 4 & 4 & 6 & 6 & 8 & 8 & 12 & 12 \\
$p^{2}$ & 31 & 1 & 806 & 1 & $?$ & 1 & $?$ & 1 \\
$p^{3}$ & 1966 & 1 & $?$ & 1 & $?$ & $?$ & $?$ & $?$ \\
$p^{4}$ & $?$ & 4 & $?$ & $?$ & $?$ & $?$ & $?$ & $?$ \\
$p^{5}$ & $?$ & 1 & $?$ & $?$ & $?$ & $?$ & $?$ & $?$ \\
$p^{6}$ & $?$ & 1 & $?$ & $?$ & $?$ & $?$ & $?$ & $?$ \\
$p^{7}$ & $?$ & 4 & $?$ & $?$ & $?$ & $?$ & $?$ & $?$ \\
\hline
\end{tabular}

Here '?' denotes an index where our computations did not terminate within a reasonable amount of time. The only normal subgroups with index $p^{2}$ are the derived subgroups 
since $\Gamma_{p} / \Gamma_{p}^{\prime} \cong \mathbb{Z}_{p} \times \mathbb{Z}_{p}$ holds [57]. For a prime power index $d=p^{\ell}$, the groups $\Gamma_{d}$ only admit subgroups with prime power index $p^{j}$ :

\begin{tabular}{ccccccc}
\hline \multirow{3}{*}{ index } & \multicolumn{2}{c}{$p^{\ell}=2^{2}$} & \multicolumn{2}{c}{$p^{\ell}=2^{3}$} & \multicolumn{2}{c}{$p^{\ell}=3^{2}$} \\
& $\leq$ & $\unlhd$ & $\leq$ & $\unlhd$ & $\leq$ & $\unlhd$ \\
\hline$p^{0}$ & 1 & 1 & 1 & 1 & 1 & 1 \\
$p^{1}$ & 3 & 3 & 3 & 3 & 4 & 4 \\
$p^{2}$ & 19 & 7 & 19 & 7 & 76 & 13 \\
$p^{3}$ & 211 & 7 & 163 & 19 & $?$ & $?$ \\
$p^{4}$ & 2419 & 11 & 2227 & 23 & $?$ & $?$ \\
\hline
\end{tabular}

For the groups $\Gamma_{6}$ and $\Gamma_{10}$, we obtain the following subgroup counts:

\begin{tabular}{ccccc}
\hline \multirow{2}{*}{ index } & \multicolumn{2}{c}{$\Gamma_{6}$} & \multicolumn{2}{c}{$\Gamma_{10}$} \\
& $\leq$ & $\unlhd$ & $\leq$ & $\unlhd$ \\
\hline 1 & 1 & 1 & 1 & 1 \\
2 & 3 & 3 & 3 & 3 \\
3 & 7 & 4 & 0 & 0 \\
4 & 9 & 1 & 5 & 1 \\
5 & 0 & 0 & 11 & 6 \\
6 & 39 & 13 & 0 & 0 \\
7 & 0 & 0 & 0 & 0 \\
8 & 45 & 1 & 1 & 1 \\
9 & 79 & 1 & 0 & 0 \\
10 & 0 & 0 & 113 & 19 \\
\hline
\end{tabular}

\begin{tabular}{ccccc}
\hline index & \multicolumn{2}{c}{$\Gamma_{6}$} & \multicolumn{2}{c}{$\Gamma_{10}$} \\
& $\leq$ & $\unlhd$ & $\leq$ & $\unlhd$ \\
\hline 11 & 0 & 0 & 0 & 0 \\
12 & 219 & 6 & 0 & 0 \\
13 & 0 & 0 & 0 & 0 \\
14 & 0 & 0 & 0 & 0 \\
15 & 0 & 0 & 0 & 0 \\
16 & 188 & 0 & 16 & 0 \\
17 & 0 & 0 & 0 & 0 \\
18 & 1299 & 7 & 0 & 0 \\
19 & 0 & 0 & 0 & 0 \\
20 & 0 & 0 & $?$ & $?$ \\
\hline
\end{tabular}

\section{A.6 Computing Solvable Quotients}

The coset enumeration process in [67] was used to prove the following version of the Reidemeister-Schreier theorem for finitely presented groups in [70]:

Theorem 1.10 Each finite-index subgroup of a finitely L-presented group is finitely Lpresented.

Proof. For a constructive proof, we refer to [70].

The constructive proof of Theorem 1.10 allows us to apply the method for finitely $L$ presented groups to finite index subgroups of a finitely $L$-presented group. As an application of this method, we consider the successive quotients $G / G^{(i)}$ of the derived series. This series is defined recursively by $G^{(1)}=G^{\prime}=[G, G]$ and $G^{(i+1)}=\left[G^{(i)}, G^{(i)}\right]$ for $i \in \mathbb{N}$. The isomorphism type of the abelian quotient $G / G^{\prime}$ can be computed with the methods from [9,64 provided that $G$ is given by a finite $L$-presentation. Moreover, it is decidable whether or not $G^{\prime}$ has finite index in $G$; see [9, 64]. 
Suppose that $G / G^{\prime}$ is finite. Then the constructive proof of Theorem 1.10 allows us to compute a finite $L$-presentation for the finite index subgroup $G^{\prime} \leq G$. Then we can compute its abelianization and we can continue this process. In general, if $G / G^{(i+1)}$ is finite, we can therefore compute the quotients $G^{(i+1)} / G^{(i+2)}$ recursively. An alternative approach to compute the sections $G^{(i)} / G^{(i+1)}$ could generalize the methods for finitely presented groups 92.

For the Grigorchuk group $\mathfrak{G}$, the sections $G^{(i)} / G^{(i+1)}$ of the derived series have been computed by Rozhkov [122]; see also [134]:

Theorem 1.11 (Rozhkov [122]) The Grigorchuk group $\mathfrak{G}$ satisfies $\left[\mathfrak{G}: \mathfrak{G}^{\prime}\right]=2^{3}$, $\left[\mathfrak{G}: \mathfrak{G}^{\prime \prime}\right]=2^{7}$, and $\left[\mathfrak{G}: \mathfrak{G}^{(k)}\right]=2^{2+2^{2 k-2}}$ for $k \geq 3$.

Our implementation of the Reidemeister-Schreier Theorem 1.10 yields that

$$
\mathfrak{G} / \mathfrak{G}^{\prime} \cong\left(\mathbb{Z}_{2}\right)^{3}, \quad \mathfrak{G}^{\prime} / \mathfrak{G}^{\prime \prime} \cong \mathbb{Z}_{2} \times \mathbb{Z}_{2} \times \mathbb{Z}_{4}, \quad \text { and } \quad \mathfrak{G}^{\prime \prime} / \mathfrak{G}^{(3)} \cong\left(\mathbb{Z}_{2}\right)^{2} \times\left(\mathbb{Z}_{4}\right)^{3} \times \mathbb{Z}_{8}
$$

Since the abelianization $\Gamma_{p} / \Gamma_{p}^{\prime} \cong \mathbb{Z}_{p} \times \mathbb{Z}_{p}$ of the Fabrykowski-Gupta group $\Gamma_{p}$ is finite [57], the derived subgroup $\Gamma_{p}^{\prime}$ satisfies $\left[\Gamma_{p}: \Gamma_{p}^{\prime}\right]=p^{2}$. A finite $L$-presentation for $\Gamma_{p}^{\prime}$ can be computed with the methods in [70]. We obtain that

$$
\Gamma_{3}^{\prime} / \Gamma_{3}^{\prime \prime} \cong\left(\mathbb{Z}_{3}\right)^{2}, \quad \Gamma_{3}^{\prime \prime} / \Gamma_{3}^{(3)} \cong\left(\mathbb{Z}_{3}\right)^{4}, \quad \text { and } \quad \Gamma_{3}^{(3)} / \Gamma_{3}^{(4)} \cong\left(\mathbb{Z}_{3}\right)^{10}
$$

as well as $\Gamma_{4}^{\prime} / \Gamma_{4}^{\prime \prime} \cong\left(\mathbb{Z}_{4}\right)^{2}$,

$$
\Gamma_{4}^{\prime \prime} / \Gamma_{4}^{(3)} \cong \mathbb{Z}_{2} \times\left(\mathbb{Z}_{4}\right)^{2} \times \mathbb{Z}_{8}, \quad \text { and } \quad \Gamma_{4}^{(3)} / \Gamma_{4}^{(4)} \cong\left(\mathbb{Z}_{2}\right)^{3} \times\left(\mathbb{Z}_{4}\right)^{9} \times\left(\mathbb{Z}_{8}\right)^{3}
$$

For $5 \leq d \leq 41$, our computations suggest the following

Proposition 1.12 For $d \geq 5, \Gamma_{d}$ satisfies $\Gamma_{d} / \Gamma_{d}^{\prime} \cong\left(\mathbb{Z}_{d}\right)^{2}$ and $\Gamma_{d}^{\prime} / \Gamma_{d}^{\prime \prime} \cong\left(\mathbb{Z}_{d}\right)^{d-1}$.

Proof. It was already shown in [57] that $\Gamma_{d} / \Gamma_{d}^{\prime} \cong \mathbb{Z}_{d} \times \mathbb{Z}_{d}$ holds. For the second statement, we combine the methods from [49] and [57]: For primes $p$, the structure of the congruence subgroups $\Gamma_{p} / \operatorname{Stab}_{\Gamma_{p}}(n), n \in \mathbb{N}$, were studied in [49]. Moreover, it was shown in [57] that, for $d \geq 5$, the index $\left[\Gamma_{d}^{\prime}: \Gamma_{d}^{\prime \prime}\right]$ is finite.

Let $d \geq 5$ be given. Denote by $\operatorname{Stab}_{\Gamma_{d}}(1)$ the first level stabilizer in $\Gamma_{d}$. Then $\Gamma_{d}=\operatorname{Stab}_{\Gamma_{d}}(1) \rtimes\langle a\rangle$ and $\operatorname{Stab}_{\Gamma_{d}}(1)=\left\langle r, r^{a}, \ldots, r^{a^{d-1}}\right\rangle$ hold. Since $\Gamma_{d}^{\prime}=\langle[a, r]\rangle^{\Gamma_{d}}=$ $\left\langle r^{-a} r\right\rangle^{\Gamma_{d}}$, we have that $\Gamma_{d}^{\prime} \leq \operatorname{Stab}_{\Gamma_{d}}(1)$ and, as $\Gamma_{d} / \Gamma_{d}^{\prime} \cong \mathbb{Z}_{d} \times \mathbb{Z}_{d}$ holds, we have that $\left[\operatorname{Stab}_{\Gamma_{d}}(1): \Gamma_{d}^{\prime}\right]=d$. More precisely, we have $\operatorname{Stab}_{\Gamma_{d}}(1)=\Gamma_{d}^{\prime} \rtimes\langle r\rangle$.

For each $0 \leq i<d$, we write $g_{i}=r^{a^{i}}$. In the following, indices are read modulo $d$. For $0 \leq \ell<d$, $g_{i}^{\ell}$ decomposes as $\left(1, \ldots, 1, r^{\ell}, a^{\ell}, 1, \ldots, 1\right)$ where $a^{\ell}$ is at position $i$. If $|\ell-k|>1$, the commutator $\left[g_{i}^{\ell}, g_{j}^{k}\right]$ is trivial; otherwise, the commutator $\left[g_{i}^{\ell}, g_{i+1}^{k}\right]$ decomposes as $\left(1, \ldots, 1,\left[a^{\ell}, r^{k}\right], 1, \ldots, 1\right)$ with $\left[a^{\ell}, r^{k}\right]$ at position $i$. Since $\left[a^{\ell}, r^{k}\right] \in \operatorname{Stab}_{\Gamma_{d}}(1)$, we have that $\left[g_{i}^{\ell}, g_{j}^{k}\right] \in \operatorname{Stab}_{\Gamma_{d}}(2)$. Thus, $\operatorname{Stab}_{\Gamma_{d}}(1) / \operatorname{Stab}_{\Gamma_{d}}(2)$ is abelian and it is generated by the images of the elements $g_{0}, \ldots, g_{d-1}$. Because $\left[a^{\ell}, r^{k}\right]=a^{-\ell} r^{-k} a^{\ell} r^{k}=g_{\ell}^{-k} g_{0}^{k}$, we have that $\left[g_{i}^{\ell}, g_{j}^{k}\right] \in \operatorname{Stab}_{\Gamma_{d}}(3)$ if and only if $\ell k \equiv 0$ 
$(\bmod d)$. Therefore $\operatorname{Stab}_{\Gamma_{d}}(1) / \operatorname{Stab}_{\Gamma_{d}}(2) \cong \mathbb{Z}_{d} \times \cdots \times \mathbb{Z}_{d}$ and $\Gamma_{d} / \operatorname{Stab}_{\Gamma_{d}}(2) \cong \mathbb{Z}_{d} \prec \mathbb{Z}_{d}$ Since $\operatorname{Stab}_{\Gamma_{d}}(1) / \operatorname{Stab}_{\Gamma_{d}}(2)$ is abelian, we have that $\operatorname{Stab}_{\Gamma_{d}}(1)^{\prime} \leq \operatorname{Stab}_{\Gamma_{d}}(2)$. Because each generator of $\operatorname{Stab}_{\Gamma_{d}}(1)$ has order $d$, the largest abelian quotient $\operatorname{Stab}_{\Gamma_{d}}(1) / \operatorname{Stab}_{\Gamma_{d}}(1)^{\prime}$ has order at most $d^{d}$. It follows that $\operatorname{Stab}_{\Gamma_{d}}(2)=\operatorname{Stab}_{\Gamma_{d}}(1)^{\prime}$. Moreover, we have $\operatorname{Stab}_{\Gamma_{d}}(2)=\operatorname{Stab}_{\Gamma_{d}}(1)^{\prime} \leq \Gamma_{d}^{\prime}$ and, since $\Gamma_{d}^{\prime} \leq \operatorname{Stab}_{\Gamma_{d}}(1)$ holds, it follows that $\Gamma_{d}^{\prime \prime} \leq$ $\operatorname{Stab}_{\Gamma_{d}}(1)^{\prime}=\operatorname{Stab}_{\Gamma_{d}}(2)$. The proofs in $\left[9,57\right.$ yield that $\operatorname{Stab}_{\Gamma_{d}}(2) \leq \Gamma_{d}^{\prime \prime}$ if $d \geq 5$. Therefore $d^{d-1}=\left|\Gamma_{d}^{\prime} / \operatorname{Stab}_{\Gamma_{d}}(2)\right|=\left|\Gamma_{d}^{\prime} / \Gamma_{d}^{\prime \prime}\right|$ and $\Gamma_{d}^{\prime} / \Gamma_{d}^{\prime \prime} \cong \mathbb{Z}_{d} \times \cdots \times \mathbb{Z}_{d}$.

The constructive proof of Theorem 1.10 in [70] yields a finite $L$-presentation over the Schreier generators of the subgroup. By the Nielsen-Schreier theorem (as, for instance, in [121, 6.1.1]), a subgroup $H$ with index $m=[G: H]$ in an $n$-generated finitely $L$ presented group $G$ has $n m+1-m$ Schreier generators. The Fabrykowski-Gupta groups are 2 -generated and therefore, the subgroup $\Gamma_{3}^{(3)}$ satisfies $\left[\Gamma_{3}: \Gamma_{3}^{(3)}\right]=3^{16}$. Thus $\Gamma_{3}^{(3)}$ has $3^{16}-1$ Schreier generators as a subgroup of the 2 -generated group $\Gamma_{3}$. Therefore, computing the sections $\Gamma_{3}^{(i)} / \Gamma_{3}^{(i+1)}, i \geq 4$, with the above method is hard in practice.

\section{Acknowledgments}

I am grateful to Laurent Bartholdi for valuable comments and suggestions.

René Hartung, Mathematisches Institut, Georg-August Universität zu Göttingen, Bunsenstrasse 3-5, 37073 Göttingen, Germany

Email: $\quad$ rhartung@uni-math.gwdg.de 


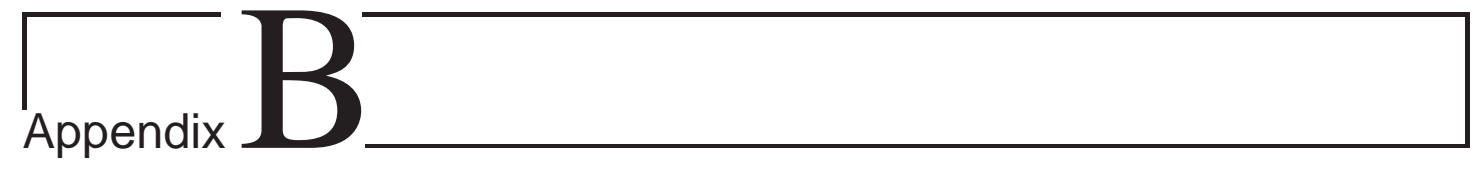

\title{
A Note on Invariantly Finitely $L$-Presented Groups
}

\begin{abstract}
In the first part of this note, we introduce Tietze transformations for $L$-presentations. These transformations enable us to generalize Tietze's theorem for finitely presented groups to invariantly finitely $L$-presented groups. Moreover, they allow us to prove that 'being invariantly finitely $L$-presented' is an abstract property of a group which does not depend on the generating set.

In the second part of this note, we consider finitely generated normal subgroups of finitely presented groups. Benli proved that a finitely generated normal subgroup of a finitely presented group is invariantly finitely $L$-presented whenever its quotient is infinite cyclic. We generalize this result to the case where the finitely presented group splits over its finitely generated subgroup and to the case where the quotient is abelian with torsion-free rank at most two.
\end{abstract}

Keywords. Tietze transformations; infinite presentations; recursive presentations; self-similar groups.

Mathematics Subject Classification 2010: 20F05, 20E07, 20-04

\section{B.1 Introduction}

Finite $L$-presentations are possibly infinite group presentations with finitely many generators whose relations (up to finitely many exceptions) are obtained by iteratively applying finitely many substitutions to a finite set of relations; see [6] or Section B.2 for a definition. Various infinitely presented groups can be described by a finite $L$-presentation. For example, the Grigorchuk group [53] and the Gupta-Sidki group [62] are finitely $L$ presented [6,9,96,128]. An $L$-presentation is invariant if the substitutions, which generate the relations, induce endomorphisms of the group. In fact, invariant finite $L$-presentations are finite presentations in the universe of groups with operators [87,111] in the sense that the operator domain of the group generates the possibly infinitely many relations out of 
a finite set of relations. The finite $L$-presentation for the Grigorchuk group in [96] is an example of an invariant finite $L$-presentation [55].

Finite $L$-presentations allow computer algorithms to be applied in the investigation of the groups they define. For instance, they allow one to compute the lower central series quotients [9], to compute the Dwyer quotients of the group's Schur multiplier [66], to develop a coset enumerator for finite index subgroups [67, and even the Reidemeister-Schreier theorem for finitely presented groups generalizes to finitely $L$-presented groups [70]. For a survey on the application of computers in the investigation of finitely $L$-presented groups, we refer to [68.

In the first part of this note, we introduce Tietze transformations for $L$-presentations. These transformations allow us to generalize Tietze's theorem for finitely presented groups [132] to invariantly finitely $L$-presented groups:

Theorem A Two invariant finite L-presentations define isomorphic groups if and only if it is possible to pass from one L-presentation to the other by a finite sequence of transformations.

If a group admits a finite presentation with respect to one generating set, then so it does with respect to any other finite generating set [38, Chapter V]. This result for finitely presented groups also generalizes to invariant finite $L$-presentations:

Theorem B (Bartholdi [6]) Being invariantly finitely L-presented is an abstract property of a group which does not depend on the generating set.

Our proof of Theorem B fills a gap in the proof of [6, Proposition 2.2] because the transformations in the latter proof are not sufficient; see Section B.4 below.

In the second part of this note, in Section B.5, we consider finitely generated normal subgroups of finitely presented groups. By Higman's embedding theorem, every finitely generated group embeds into a finitely presented group if and only if it is recursively presented [81]. Since every finite $L$-presentation is recursive, finitely $L$-presented groups therefore embed into finitely presented groups. As indicated in [22], we prove that every group which admits an invariant finite $L$-presentation, where each substitution induces an automorphism of the group, embeds as a normal subgroup into a finitely presented group. On the other hand, the Reidemeister-Schreier theorem for finitely $L$-presented groups in [70] shows that every normal subgroup of a finitely presented group admits an invariant $L$-presentation where each substitution induces an automorphism of the group; the obtained $L$-presentation is finite if and only if the normal subgroup has finite index.

Finitely generated normal subgroups of finitely presented groups with infinite index were considered in 22]: It was proved that a finitely generated normal subgroup of a finitely presented group is invariantly finitely $L$-presented if its quotient is infinite cyclic. Moreover, in [22, Remark (2)], Benli asked for a generalization of his latter result and he posed the following problem:

Is it true that a finitely generated group embeds as a normal subgroup into a finitely presented group if and only if it admits an invariant finite Lpresentation where each substitution induces an automorphism of the group? 
We generalize Benli's constructions from [22] in order to prove the following

Theorem C Every finitely generated normal subgroup of a finitely presented group is invariantly finitely L-presented if the group splits over its subgroup.

Since $G$ splits over its subgroup $H \unlhd G$ if $G / H$ is a free group, Benli's result in [22] is a consequence of Theorem C. Moreover, our generalizations of the constructions from [22] allow us to prove

Theorem D Every finitely generated normal subgroup of a finitely presented group is invariantly finitely L-presented whenever the quotient is abelian with torsion-free rank at most two.

Our constructions do not generalize further; see Remark 2.1.

\section{B.2 Preliminaries}

In this section, we recall the notion of an invariant finite $L$-presentation as introduced in [6]. An L-presentation is a group presentation of the form

$$
\left\langle\mathcal{X} \mid \mathcal{Q} \cup \bigcup_{\sigma \in \Phi^{*}} \mathcal{R}^{\sigma}\right\rangle
$$

where $\mathcal{X}$ is an alphabet, $\mathcal{Q}$ and $\mathcal{R}$ are subsets of the free group $F=F(\mathcal{X})$ over the alphabet $\mathcal{X}$, and $\Phi^{*} \subseteq \operatorname{End}(F)$ denotes the monoid of endomorphisms that is generated by $\Phi$. If the generators $\mathcal{X}$, the fixed relations $\mathcal{Q}$, the substitutions $\Phi$, and the iterated relations $\mathcal{R}$ have finite cardinality, the $L$-presentation in Eq. (B.1) is a finite L-presentation. We also write $\langle\mathcal{X}|\mathcal{Q}| \Phi \mid \mathcal{R}\rangle$ for the $L$-presentation in Eq. (B.1) and $G=\langle\mathcal{X}|\mathcal{Q}| \Phi \mid \mathcal{R}\rangle$ for the group it defines.

A group which admits a finite $L$-presentation is finitely $L$-presented. An $L$-presentation of the form $\langle\mathcal{X}|\emptyset| \Phi \mid \mathcal{R}\rangle$ is ascending and an $L$-presentation $\langle\mathcal{X}|\mathcal{Q}| \Phi \mid \mathcal{R}\rangle$ is called invariant (and the group it defines is invariantly L-presented), if each substitution $\varphi \in \Phi$ induces an endomorphism of the group; i.e., if the normal subgroup $\left\langle\mathcal{Q} \cup \bigcup_{\sigma \in \Phi^{*}} \mathcal{R}^{\sigma}\right\rangle^{F} \unlhd F$ is $\varphi$-invariant. Each ascending $L$-presentation is invariant and each invariant $L$-presentation $\langle\mathcal{X}|\mathcal{Q}| \Phi \mid \mathcal{R}\rangle$ admits an ascending $L$-presentation $\langle\mathcal{X}|\emptyset| \Phi \mid \mathcal{Q} \cup \mathcal{R}\rangle$ which defines the same group; see Proposition 3.7, Even though invariant and ascending $L$-presentations are essentially the same, we like to distinguish between these two objects. The finite $L$-presentation in [96] for the group constructed by Grigorchuk [53] is not ascending but it is easy to see that it is an invariant $L$-presentation; see, for instance, [55, Corollary 4].

Remark 2.1 There are finite L-presentations that are not invariant.

Proof. The free product $\mathbb{Z}_{2} * \mathbb{Z}_{2}=\left\langle\{a, b\} \mid\left\{a^{2}, b^{2}\right\}\right\rangle$ is finitely $L$-presented by $\left\langle\{a, b\}\left|\left\{a^{2}\right\}\right|\{\sigma\} \mid\left\{b^{2}\right\}\right\rangle$ where $\sigma$ is induced by the map $a \mapsto a b$ and $b \mapsto b^{2}$. If this $L$-presentation were invariant, the ascending $L$-presentation $\left\langle\{a, b\}|\emptyset|\{\sigma\} \mid\left\{a^{2}, b^{2}\right\}\right\rangle$ 
would also define $\mathbb{Z}_{2} * \mathbb{Z}_{2}$; see Proposition 3.7. In this case $\left(a^{2}\right)^{\sigma}=a b a b$ is a relation in the group and, since $a^{2}=b^{2}=1$ holds, the generators $a$ and $b$ commute. Therefore the ascending $L$-presentation defines a quotient of the 2-elementary abelian group $\mathbb{Z}_{2} \times \mathbb{Z}_{2}$. In fact, it defines a finite group. Thus $\left\langle\{a, b\}|\emptyset|\{\sigma\} \mid\left\{a^{2}, b^{2}\right\}\right\rangle$ is not a finite $L$-presentation for $\mathbb{Z}_{2} * \mathbb{Z}_{2}$ and hence $\left\langle\{a, b\}\left|\left\{a^{2}\right\}\right|\left\{\sigma^{2}\right\} \mid\left\{b^{2}\right\}\right\rangle$ is not an invariant $L$-presentation.

Note that this latter proof from [70] provides a 'method' to prove that a finite $L$ presentation $\langle\mathcal{X}|\mathcal{Q}| \Phi \mid \mathcal{R}\rangle$ is invariant; namely, if the ascending $L$-presentation $\langle\mathcal{X}|\emptyset| \Phi \mid \mathcal{R} \cup \mathcal{Q}\rangle$ defines a group which is isomorphic to the first. In general, we are not aware of a method which allows us to decide whether or not a finite $L$-presentation is invariant - even if we assume that the $L$-presented group has a solvable word problem.

Invariant finite $L$-presentations are 'natural' generalizations of finite presentations because every finitely presented group $\langle\mathcal{X} \mid \mathcal{R}\rangle$ is invariantly finitely $L$-presented by $\langle\mathcal{X}|$ $\emptyset|\emptyset| \mathcal{R}\rangle$. However, invariant finite $L$-presentations have been used to describe various examples of self-similar groups that are not finitely presented [14, 96]. For instance, the group $\mathfrak{G}$ constructed by Grigorchuk in [53] is not finitely presented [56] but it is invariantly finitely $L$-presented, see also [55]:

Theorem 2.2 (Lysënok [96]) The Grigorchuk group is invariantly finitely L-presented by $\left.\left\langle\{a, b, c, d\}\left|\left\{a^{2}, b^{2}, c^{2}, d^{2}, b c d\right\}\right|\{\sigma\}\right|\left\{(a d)^{4},(\text { adacac })^{4}\right\}\right\rangle$ where $\sigma$ denotes the endomorphism of the free group over $\{a, b, c, d\}$ that is induced by the map $a \mapsto a c a, b \mapsto d$, $c \mapsto b$, and $d \mapsto c$.

It is easy to see (and it follows with our Tietze transformations below) that the group $\mathfrak{G}$ is also invariantly finitely $L$-presented by

$$
\left.\mathfrak{G} \cong\left\langle\{a, c, d\}\left|\left\{a^{2}, c^{2}, d^{2},(c d)^{2}\right\}\right|\{\tilde{\sigma}\}\right|\left\{(a d)^{4},(\text { adacac })^{4}\right\}\right\rangle,
$$

where $\tilde{\sigma}$ is induced by the map $a \mapsto a c a, c \mapsto c d$, and $d \mapsto c$. Further examples of invariantly finitely $L$-presented groups arise, for instance, as certain wreath-products: In contrast to [6], Bartholdi noticed that the lamplighter group $\mathbb{Z}_{2} \prec \mathbb{Z}$ is invariantly finitely $L$-presented by

$$
\left\langle\{a, t\}|\emptyset|\{\delta\} \mid\left\{a^{2},\left[a, a^{t}\right]\right\}\right\rangle,
$$

where $\delta$ is induced by the map $a \mapsto a^{t} a$ and $t \mapsto t$. This recent result generalizes to wreath products of the form $H \imath \mathbb{Z}$, where $H$ is a finitely generated abelian group:

Proposition 2.3 If $H$ is a finitely generated abelian group, the wreath product $H \prec \mathbb{Z}$ is invariantly finitely L-presented.

Proof. Since $H$ is finitely generated and abelian, it decomposes into a direct product of cyclic groups; i.e., $H$ has the form $\mathbb{Z}_{r_{1}} \times \cdots \times \mathbb{Z}_{r_{n}}$ for $r_{1}, \ldots, r_{n} \in \mathbb{N} \cup\{\infty\}$ where $\mathbb{Z}_{\infty}$ denotes the infinite cyclic group while $\mathbb{Z}_{r_{i}}$ denotes the cyclic group of order $r_{i}$, otherwise. Then $\left\langle\mathcal{X} \mid\{[x, y] \mid x, y \in \mathcal{X}\} \cup\left\{x^{r_{x}} \mid r_{x}<\infty\right\}\right\rangle$ is a finite presentation for $H$. The wreath product $H \prec \mathbb{Z}$ admits the presentation

$$
H\left\langle\mathbb{Z} \cong\left\langle\mathcal{X} \cup\{t\} \mid\left\{[x, y], x^{r_{x}}\right\}_{x, y \in \mathcal{X}, r_{x}<\infty} \cup\left\{\left[x, y^{t^{i}}\right]\right\}_{x, y \in \mathcal{X}, i \in \mathbb{N}_{0}}\right\rangle .\right.
$$


For each $y \in \mathcal{X}$, define a substitution $\sigma_{y}$ which is induced by the map

$$
\sigma_{y}:\left\{\begin{aligned}
y & \mapsto y^{t} y, \\
x & \mapsto x, \\
t & \mapsto t .
\end{aligned} \text { for each } x \in \mathcal{X} \backslash\{y\},\right.
$$

For $n \in \mathbb{N}$ and $x, y, z \in \mathcal{X}$ with $x \neq y$ and $z \neq y$, we obtain

$$
\begin{aligned}
{\left[y, x^{t^{n}}\right]^{\sigma_{y}} } & =\left[y^{t} y, x^{t^{n}}\right]=\left[y, x^{t^{n-1}}\right]^{t y} \cdot\left[y, x^{t^{n}}\right], \\
{\left[x, y^{t^{n}}\right]^{\sigma_{y}} } & =\left[x, y^{t^{n+1}} y^{t^{n}}\right]=\left[x, y^{t^{n}}\right] \cdot\left[x, y^{t^{n+1}}\right]^{t^{n}}, \\
{\left[x, z^{t^{n}}\right]^{\sigma_{y}} } & =\left[x, z^{t^{n}}\right], \\
{\left[y, y^{t^{n}}\right]^{\sigma_{y}} } & =\left[y, y^{t^{n-1}}\right]^{t y} \cdot\left[y, y^{t^{n}}\right]^{t t^{t^{n}} y} \cdot\left[y, y^{t^{n}}\right] \cdot\left[y, y^{t^{n+1}}\right]^{y^{t^{n}}} .
\end{aligned}
$$

This shows that the relations $\left\{\left[x, y^{t^{i}}\right] \mid x, y \in \mathcal{X}, i \in \mathbb{N}\right\}$ are consequences of the iterated images $\left\{\left[x, y^{t}\right]^{\delta} \mid \delta \in\left\{\sigma_{y} \mid y \in \mathcal{X}\right\}^{*}, x, y \in \mathcal{X}\right\}$ and vice versa. Moreover, for each relation $x^{r_{x}}$ of $H$ 's finite presentation, we have that $\left(x^{r_{x}}\right)^{\sigma_{y}}=x^{r_{x}}$ if $x \neq y$ and $\left(y^{r_{y}}\right)^{\sigma_{y}}=$ $\left(y^{t} y\right)^{r_{y}}=_{H Z \mathbb{Z}}\left(y^{r_{y}}\right)^{t} y^{r_{y}}$, otherwise. Thus these images are relations of the wreath product $H \imath \mathbb{Z}$. In particular, the finite $L$-presentation

$$
\left\langle\mathcal{X} \cup\{t\}|\emptyset|\left\{\sigma_{y}\right\}_{y \in \mathcal{X}} \mid\left\{\left[x, y^{t}\right]\right\}_{x, y \in \mathcal{X}} \cup\left\{x^{r_{x}}\right\}_{x \in \mathcal{X}, r_{x}<\infty}\right\rangle
$$

is an invariant finite $L$-presentation for the wreath product $H<\mathbb{Z}$.

Even though invariant finite $L$-presentations are known for numerous self-similar groups, we are not aware of an invariant finite $L$-presentation for the Gupta-Sidki group from 62 . Moreover, we are not aware of a finitely $L$-presented group which is not invariantly finitely $L$-presented.

\section{B.3 Tietze Transformations for $L$-Presentations}

In this section, we introduce Tietze transformations for $L$-presentations. Let $G=\langle\mathcal{X}|$ $\mathcal{Q}|\Phi| \mathcal{R}\rangle$ be an $L$-presented group. Denote by $F$ the free group $F(\mathcal{X})$ over the alphabet $\mathcal{X}$ and let $K=\left\langle\mathcal{Q} \cup \bigcup_{\sigma \in \Phi^{*}} \mathcal{R}^{\sigma}\right\rangle^{F}$ be the kernel of the free presentation $\pi: F \rightarrow G$. Then $K=\operatorname{ker} \pi$ decomposes into the normal subgroups $Q=\langle\mathcal{Q}\rangle^{F}$ and $R=\left\langle\bigcup_{\sigma \in \Phi^{*}} \mathcal{R}^{\sigma}\right\rangle^{F}$ so that $K=R Q=Q R$ holds. The group $F / R$ is invariantly $L$-presented by $\langle\mathcal{X}|\emptyset| \Phi \mid \mathcal{R}\rangle$. We can add every element of the kernel $K$ as a fixed relation:

Proposition 3.4 If $G=\langle\mathcal{X}|\mathcal{Q}| \Phi \mid \mathcal{R}\rangle$ is a (finitely) L-presented group and $\mathcal{S} \subseteq\left\langle\mathcal{Q} \cup \bigcup_{\sigma \in \Phi^{*}} \mathcal{R}^{\sigma}\right\rangle^{F}$ is a (finite) subset, then $\langle\mathcal{X}|\mathcal{Q} \cup \mathcal{S}| \Phi \mid \mathcal{R}\rangle$ is a (finite) $L$-presentation for $G$.

Proof. The proof follows with the Tietze transformation that adds consequences $\mathcal{S}$ of $G$ 's relations to the group presentation $\left\langle\mathcal{X} \mid \mathcal{Q} \cup \bigcup_{\sigma \in \Phi^{*}} \mathcal{R}^{\sigma}\right\rangle$.

The transformation in Proposition 3.4 is reversible in the sense that we can remove fixed relations $\mathcal{S}$ from an $L$-presentation $\langle\mathcal{X}|\mathcal{Q} \cup \mathcal{S}| \Phi \mid \mathcal{R}\rangle$ if and only if 
$\left\langle\mathcal{Q} \cup \mathcal{S} \cup \bigcup_{\sigma \in \Phi^{*}} \mathcal{R}^{\sigma}\right\rangle^{F}=\left\langle\mathcal{Q} \cup \bigcup_{\sigma \in \Phi^{*}} \mathcal{R}^{\sigma}\right\rangle^{F}$ holds. The following transformations are reversible in the same sense.

If an $L$-presentation is not invariant (cf. Remark 2.1), there exist elements from the kernel $K$ of the free presentation $\pi: F \rightarrow G$ that we cannot add as iterated relations without changing the isomorphism type of the group. However, even for non-invariant $L$-presentations we have the following

Proposition 3.5 If $G=\langle\mathcal{X}|\mathcal{Q}| \Phi \mid \mathcal{R}\rangle$ is a (finitely) L-presented group and $\mathcal{S} \subseteq\left\langle\bigcup_{\sigma \in \Phi^{*}} \mathcal{R}^{\sigma}\right\rangle^{F}$ is a (finite) subset, then $\langle\mathcal{X}|\mathcal{Q}| \Phi \mid \mathcal{R} \cup \mathcal{S}\rangle$ is a (finite) L-presentation for $G$.

Proof. By construction, the normal subgroup $R=\left\langle\bigcup_{\sigma \in \Phi^{*}} \mathcal{R}^{\sigma}\right\rangle^{F}$ is $\sigma$-invariant for each $\sigma \in \Phi^{*}$. More precisely, for each $r \in R$ and $\sigma \in \Phi^{*}$, we have $r^{\sigma} \in R$. Therefore, adding the (possibly infinitely many) relations $\left\{s^{\sigma} \mid s \in \mathcal{S}, \sigma \in \Phi^{*}\right\}$ to the group presentation $\left\langle\mathcal{X} \mid \mathcal{Q} \cup \bigcup_{\sigma \in \Phi^{*}} \mathcal{R}^{\sigma}\right\rangle$ does not change the isomorphism type of the group.

Iterated and fixed relations of an $L$-presentation are related by the following

Proposition 3.6 If $G=\langle\mathcal{X}|\mathcal{Q}| \Phi \mid \mathcal{R}\rangle$ is a (finitely) L-presented group and $\mathcal{S} \subseteq \mathcal{R}$ holds, then $\left\langle\mathcal{X}|\mathcal{Q} \cup \mathcal{S}| \Phi \mid(\mathcal{R} \backslash \mathcal{S}) \cup\left\{r^{\psi} \mid r \in \mathcal{S}, \psi \in \Phi\right\}\right\rangle$ is a (finite) L-presentation for $G$.

Proof. The proof follows immediately from

$$
\mathcal{Q} \cup \bigcup_{\sigma \in \Phi^{*}} \mathcal{R}^{\sigma}=\mathcal{Q} \cup \mathcal{S} \cup \bigcup_{\sigma \in \Phi^{*}}\left((\mathcal{R} \backslash \mathcal{S}) \cup\left\{r^{\psi}\right\}_{r \in \mathcal{S}, \psi \in \Phi}\right)^{\sigma} ;
$$

these are the relations of $G$ 's group presentation.

The following proposition is a consequence of the definition of an invariant $L$-presentation:

Proposition 3.7 If $\langle\mathcal{X}|\mathcal{Q}| \Phi \mid \mathcal{R}\rangle$ is an invariant (finite) L-presentation for the group $G$ and $\mathcal{S} \subseteq \mathcal{Q}$ holds, then $\langle\mathcal{X}|\mathcal{Q} \backslash \mathcal{S}| \Phi \mid \mathcal{R} \cup \mathcal{S}\rangle$ is a (finite) L-presentation for $G$.

Proof. Since $G$ is invariantly $L$-presented by $\langle\mathcal{X}|\mathcal{Q}| \Phi \mid \mathcal{R}\rangle$, each $\sigma \in \Phi$ induces an endomorphism of the group $G$. Therefore, the images $\left\{q^{\sigma} \mid q \in \mathcal{S}, \sigma \in \Phi^{*}\right\}$ are relations within $G$ and so $\left\langle\mathcal{X} \mid(\mathcal{Q} \backslash \mathcal{S}) \cup \bigcup_{\sigma \in \Phi^{*}}(\mathcal{R} \cup \mathcal{S})^{\sigma}\right\rangle$ is a presentation for $G$.

The following proposition allows us to add generators together with fixed relations to an $L$-presentation:

Proposition 3.8 Let $G=\langle\mathcal{X}|\mathcal{Q}| \Phi \mid \mathcal{R}\rangle$ be an L-presented group, $\mathcal{Z}$ be an alphabet so that $\mathcal{X} \cap \mathcal{Z}=\emptyset$ holds, and, for each $z \in \mathcal{Z}$, let $w_{z} \in F(\mathcal{X})$ be given. For each $\sigma \in \Phi$, define an endomorphism of the free group $E$ over the alphabet $\mathcal{X} \cup \mathcal{Z}$ that is induced by the map

$$
\tilde{\sigma}:\left\{\begin{aligned}
x & \mapsto x^{\sigma}, \quad \text { for each } x \in \mathcal{X} \\
z & \mapsto g_{z}, \quad \text { for each } z \in \mathcal{Z}
\end{aligned}\right.
$$


where $g_{z}$ are arbitrary elements of the free group $E$. Then $G$ satisfies that

$$
G \cong\left\langle\mathcal{X} \cup \mathcal{Z}\left|\mathcal{Q} \cup\left\{z^{-1} w_{z}\right\}_{z \in \mathcal{Z}}\right|\{\tilde{\sigma}\}_{\sigma \in \Phi} \mid \mathcal{R}\right\rangle .
$$

If $\langle\mathcal{X}|\mathcal{Q}| \Phi \mid \mathcal{R}\rangle$ is a finite L-presentation and $\mathcal{Z}$ is a finite alphabet, the L-presentation in $E q$. B.4 is finite.

Proof. Write $H=\left\langle\mathcal{X} \cup \mathcal{Z}\left|\mathcal{Q} \cup\left\{z^{-1} w_{z} \mid z \in \mathcal{Z}\right\}\right|\{\tilde{\sigma} \mid \sigma \in \Phi\} \mid \mathcal{R}\right\rangle$ and let $F$ and $E$ be the free groups over $\mathcal{X}$ and $\mathcal{X} \cup \mathcal{Z}$, respectively. To avoid confusion, the elements of $G^{\prime}$ 's presentation are denoted by $\bar{g} \in F$. Then

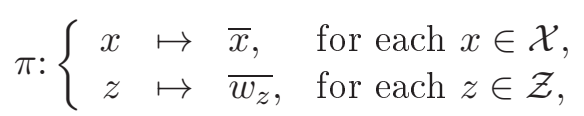

induces a surjective homomorphism $\pi: E \rightarrow F$. By construction, the restriction of the substitution $\tilde{\sigma}$ to the free group $F$ coincides with $\sigma$. Thus $\left(\bigcup_{\sigma \in \Phi} \mathcal{R}^{\tilde{\sigma}}\right)^{\pi}=\bigcup_{\sigma \in \Phi^{*}} \mathcal{R}^{\sigma}$ and hence, $\pi$ maps iterated relations of $H$ 's $L$-presentation to iterated relations of $G$. Similarly, $\pi$ maps the fixed relations $\mathcal{Q}$ of $H$ 's $L$-presentation to fixed relations of $G$. It remains to consider the relations of the form $z^{-1} w_{z}$ with $z \in \mathcal{Z}$. However, these relations are mapped trivially by $\pi$. This shows that the homomorphism $\pi: E \rightarrow F$ induces a surjective homomorphism $\tilde{\pi}: H \rightarrow G$. On the other hand, identifying the generators of $G$ 's $L$-presentation with the generators of $H$ induces a surjective homomorphism $\varphi: G \rightarrow H$ with $\varphi \tilde{\pi}=\operatorname{id}_{H}$ and $\tilde{\pi} \varphi=\operatorname{id}_{G}$. Hence, the groups $G$ and $H$ are isomorphic. The second assertion is obvious.

We can also add the relations $\left\{z^{-1} w_{z} \mid z \in \mathcal{Z}\right\}$ in Proposition 3.8 as iterated relations to the $L$-presentation if we define the substitutions $\tilde{\sigma}$ as follows:

Proposition 3.9 Let $G=\langle\mathcal{X}|\mathcal{Q}| \Phi \mid \mathcal{R}\rangle$ be an L-presented group, $\mathcal{Z}$ be an alphabet so that $\mathcal{X} \cap \mathcal{Z}=\emptyset$ holds, and, for each $z \in \mathcal{Z}$, let $w_{z} \in F(\mathcal{X})$ be given. For each $\sigma \in \Phi$, define an endomorphism of the free group $E$ over the alphabet $\mathcal{X} \cup \mathcal{Z}$ that is induced by the map

$$
\tilde{\sigma}:\left\{\begin{aligned}
x & \mapsto x^{\sigma}, \quad \text { for each } x \in \mathcal{X} \\
z & \mapsto w_{z}^{\sigma}, \quad \text { for each } z \in \mathcal{Z}
\end{aligned}\right.
$$

Then $G$ satisfies that

$$
G \cong\left\langle\mathcal{X} \cup \mathcal{Z}|\mathcal{Q}|\{\tilde{\sigma}\}_{\sigma \in \Phi} \mid \mathcal{R} \cup\left\{z^{-1} w_{z}\right\}_{z \in \mathcal{Z}}\right\rangle .
$$

If $\langle\mathcal{X}|\mathcal{Q}| \Phi \mid \mathcal{R}\rangle$ is a finite $L$-presentation and $\mathcal{Z}$ is a finite alphabet, the L-presentation in $E q$. (B.6) is finite.

Proof. The substitutions $\tilde{\sigma}$ in Eq. (B.5) are well-defined because $w_{z} \in F(\mathcal{X})$ and $\sigma \in \operatorname{End}(F(\mathcal{X}))$ hold. By Proposition [3.6, we have that

$$
\begin{aligned}
& \left\langle\mathcal{X} \cup \mathcal{Z}|\mathcal{Q}|\{\tilde{\sigma}\}_{\sigma \in \Phi} \mid \mathcal{R} \cup\left\{z^{-1} w_{z}\right\}_{z \in \mathcal{Z}}\right\rangle \\
= & \left\langle\mathcal{X} \cup \mathcal{Z}\left|\mathcal{Q} \cup\left\{z^{-1} w_{z}\right\}_{z \in \mathcal{Z}}\right|\{\tilde{\sigma}\}_{\sigma \in \Phi} \mid \mathcal{R} \cup\left\{\left(z^{-1} w_{z}\right)^{\tilde{\sigma}}\right\}_{z \in \mathcal{Z}, \sigma \in \Phi}\right\rangle .
\end{aligned}
$$


By definition of $\tilde{\sigma}$ in Eq. (B.5), we have $\left(z^{-1}\right)^{\tilde{\sigma}}=\left(w_{z}^{\sigma}\right)^{-1}$ and $w_{z}^{\tilde{\sigma}}=w_{z}^{\sigma}$. Thus $\left(z^{-1} w_{z}\right)^{\tilde{\sigma}}=$ $\left(w_{z}^{\sigma}\right)^{-1} w_{z}^{\sigma}=1$ holds. In particular, adding the relations $\left\{\left(z^{-1} w_{z}\right)^{\tilde{\sigma}} \mid z \in \mathcal{Z}, \sigma \in \Phi\right\}$ to a group presentation does not change the isomorphism type of the group. By Proposition 3.8, we have that

$$
\begin{aligned}
G & =\langle\mathcal{X}|\mathcal{Q}| \Phi \mid \mathcal{R}\rangle \\
& \cong\left\langle\mathcal{X} \cup \mathcal{Z}\left|\mathcal{Q} \cup\left\{z^{-1} w_{z}\right\}_{z \in \mathcal{Z}}\right|\{\tilde{\sigma}\}_{\sigma \in \Phi} \mid \mathcal{R}\right\rangle \\
& =\left\langle\mathcal{X} \cup \mathcal{Z}\left|\mathcal{Q} \cup\left\{z^{-1} w_{z}\right\}_{z \in \mathcal{Z}}\right|\{\tilde{\sigma}\}_{\sigma \in \Phi} \mid \mathcal{R} \cup\left\{\left(z^{-1} w_{z}\right)^{\tilde{\sigma}}\right\}_{z \in \mathcal{Z}, \sigma \in \Phi}\right\rangle \\
& =\left\langle\mathcal{X} \cup \mathcal{Z}|\mathcal{Q}|\{\tilde{\sigma}\}_{\sigma \in \Phi} \mid \mathcal{R} \cup\left\{z^{-1} w_{z}\right\}_{z \in \mathcal{Z}}\right\rangle
\end{aligned}
$$

which proves the first assertion of Proposition 3.9 while the second is obvious.

The following proposition allows us to modify the substitutions of an $L$-presentation:

Proposition 3.10 If $G=\langle\mathcal{X}|\mathcal{Q}| \Phi \mid \mathcal{R}\rangle$ is a (finitely) L-presented group and $\Psi \subseteq \Phi$ holds, then $\left\langle\mathcal{X}|\mathcal{Q}|(\Phi \backslash \Psi) \cup\{\sigma \psi \mid \psi \in \Psi, \sigma \in \Phi\} \mid \mathcal{R} \cup \bigcup_{\psi \in \Psi} \mathcal{R}^{\psi}\right\rangle$ is a (finite) $L$-presentation for $G$.

Proof. The proof follows immediately from

$$
\mathcal{Q} \cup \bigcup_{\sigma \in \Phi^{*}} \mathcal{R}^{\sigma}=\mathcal{Q} \cup \bigcup_{\sigma \in \widehat{\Phi}^{*}}\left(\mathcal{R} \cup \bigcup_{\psi \in \Psi} \mathcal{R}^{\psi}\right)^{\sigma}
$$

where $\widehat{\Phi}=(\Phi \backslash \Psi) \cup\{\sigma \psi \mid \psi \in \Psi, \sigma \in \Phi\}$; these are the relations of $G$ 's group presentation.

Since each relation of a group presentation can be replaced by a conjugate, we can modify the substitutions of an $L$-presentation as follows:

Proposition 3.11 Let $G=\langle\mathcal{X}|\mathcal{Q}| \Phi \mid \mathcal{R}\rangle$ be a (finitely) L-presented group, $\mathcal{S} \subseteq F$ be a (finite) subset, and let $\Psi \subseteq \Phi$ be given. For each $x \in \mathcal{S}$, denote by $\delta_{x}$ the inner automorphism of the free group $F(\mathcal{X})$ that is induced by conjugation with $x$. Then

- $\left\langle\mathcal{X}|\mathcal{Q}| \Phi \cup\left\{\delta_{x} \mid x \in \mathcal{S}\right\} \mid \mathcal{R}\right\rangle$,

- $\left\langle\mathcal{X}|\mathcal{Q}|(\Phi \backslash \Psi) \cup\left\{\delta_{x} \sigma \mid x \in \mathcal{S}, \sigma \in \Psi\right\} \mid \mathcal{R}\right\rangle$, and

- $\left\langle\mathcal{X}|\mathcal{Q}|(\Phi \backslash \Psi) \cup\left\{\sigma \delta_{x} \mid x \in \mathcal{S}, \sigma \in \Psi\right\} \mid \mathcal{R}\right\rangle$

are (finite) L-presentations for $G$.

Proof. This follows because each relation of a group presentation can be replaced by a conjugate and we have $\delta_{x} \sigma=\sigma \delta_{x^{\sigma}}$ for each $\sigma \in \Phi^{*}$ and $x \in \mathcal{X}$.

Recall that the kernel $K=\left\langle\mathcal{Q} \cup \bigcup_{\sigma \in \Phi^{*}} \mathcal{R}^{\sigma}\right\rangle^{F}$ of the free presentation $\pi: F \rightarrow G$ decomposes into the normal subgroups $Q=\langle\mathcal{Q}\rangle^{F}$ and $R=\left\langle\bigcup_{\sigma \in \Phi^{*}} \mathcal{R}^{\sigma}\right\rangle^{F}$ so that $K=Q R=R Q$ holds. This decomposition yields the following 
Proposition 3.12 Let $G=\langle\mathcal{X}|\mathcal{Q}| \Phi \mid \mathcal{R}\rangle$ be a (finitely) L-presented group and let $\Psi \subseteq \operatorname{End}(F(\mathcal{X}))$ be a (finite) subset. If each $\psi \in \Psi$ induces an endomorphism of $F(\mathcal{X}) / R$, then $\langle\mathcal{X}|\mathcal{Q}| \Phi \cup \Psi \mid \mathcal{R}\rangle$ is a (finite) L-presentation for $G$.

Proof. If $\psi \in \Psi$ induces an endomorphism of $F(\mathcal{X}) / R$, the normal subgroup $R$ is $\psi$ invariant. Therefore, each image $r^{\sigma} \in F(\mathcal{X})$, with $\sigma \in(\Phi \cup \Psi)^{*} \backslash \Phi^{*}$ and $r \in \mathcal{R}$, is a relation of the group. Adding these (possibly infinitely many) relations to the group presentation does not change the isomorphism type of the group.

For an invariant $L$-presentation, we even have the following

Proposition 3.13 Let $G=\langle\mathcal{X}|\mathcal{Q}| \Phi \mid \mathcal{R}\rangle$ be a (finitely) L-presented group and let $\Psi \subseteq \operatorname{End}(F(\mathcal{X}))$ be a (finite) subset. Then $\langle\mathcal{X}|\mathcal{Q}| \Phi \cup \Psi \mid \mathcal{R}\rangle$ is a (finite) L-presentation for $G$ if and only if each $\psi \in \Psi$ induces an endomorphism of $G$.

Proof. Let $K=\left\langle\mathcal{Q} \cup \bigcup_{\sigma \in \Phi^{*}} \mathcal{R}^{\sigma}\right\rangle^{F}$ be the kernel of the free presentation $\pi: F(\mathcal{X}) \rightarrow$ $G$. If each $\psi \in \Psi$ induces an endomorphism of $F(\mathcal{X}) / K$, Proposition 3.12 shows the first assertion. If, on the other hand, the invariant $L$-presentations $\langle\mathcal{X}|\mathcal{Q}| \Phi \mid \mathcal{R}\rangle$ and $\langle\mathcal{X}|\mathcal{Q}| \Phi \cup \Psi \mid \mathcal{R}\rangle$ are $L$-presentations for $G$, each $\psi \in \Psi$ induces an endomorphism of $G=F(\mathcal{X}) / K$.

Every substitution $\sigma \in \Phi$ of an invariant $L$-presentation $G=\langle\mathcal{X}|\mathcal{Q}| \Phi \mid \mathcal{R}\rangle$ induces an endomorphism of $G$. However, there are possibly other endomorphisms of the free group $F(\mathcal{X})$ that will induce the same endomorphism on $G$. The following proposition allows us to modify a given substitution of an $L$-presentation:

Proposition 3.14 Let $G=\langle\mathcal{X}|\mathcal{Q}| \Phi \mid \mathcal{R}\rangle$ be a (finitely) L-presented group, $\mathcal{S} \subseteq\left\langle\bigcup_{\varphi \in \Phi^{*}} \mathcal{R}^{\varphi}\right\rangle^{F}$ be a (finite) subset, and let $\sigma \in \Phi$ be given. Define an endomorphism $\tilde{\sigma}$ of the free group $F=F(\mathcal{X})$ over the alphabet $\mathcal{X}$ that is induced by the map $\tilde{\sigma}: x \mapsto x^{\sigma} r_{x}$ for each $x \in \mathcal{X}$ and some $r_{x} \in \mathcal{S}$. Then $\langle\mathcal{X}|\mathcal{Q}|(\Phi \backslash\{\sigma\}) \cup\{\tilde{\sigma}\} \mid \mathcal{R} \cup \mathcal{S}\rangle$ is a (finite) L-presentation for $G$.

Proof. We work in the free group $F=F(\mathcal{X})$ over the alphabet $\mathcal{X}$ and we decompose the kernel $K=\left\langle\mathcal{Q} \cup \bigcup_{\varphi \in \Phi^{*}} \mathcal{R}^{\varphi}\right\rangle^{F}$ of the free presentation $\pi: F \rightarrow G$ into the normal subgroups $Q=\langle\mathcal{Q}\rangle^{F}$ and $R=\left\langle\bigcup_{\varphi \in \Phi^{*}} \mathcal{R}^{\varphi}\right\rangle^{F}$ as above. Since $\mathcal{S} \subseteq\left\langle\bigcup_{\varphi \in \Phi^{*}} \mathcal{R}^{\varphi}\right\rangle^{F}$ holds, Proposition 3.5 yields that $G=\langle\mathcal{X}|\mathcal{Q}| \Phi \mid \mathcal{R}\rangle=\langle\mathcal{X}|\mathcal{Q}| \Phi \mid \mathcal{R} \cup \mathcal{S}\rangle$. In particular, we have that $R=\left\langle\bigcup_{\varphi \in \Phi^{*}}(\mathcal{R} \cup \mathcal{S})^{\varphi}\right\rangle^{F}$. Write $\Psi=(\Phi \backslash\{\sigma\}) \cup\{\tilde{\sigma}\}$. We prove this proposition by showing that the normal subgroups $R=\left\langle\bigcup_{\varphi \in \Phi^{*}}(\mathcal{R} \cup \mathcal{S})^{\varphi}\right\rangle^{F}$ and $\tilde{R}=$ $\left\langle\bigcup_{\varphi \in \Psi^{*}}(\mathcal{R} \cup \mathcal{S})^{\varphi}\right\rangle^{F}$ coincide. For this purpose, we prove that, for each $\tilde{\delta} \in \Psi^{*}$ and $g \in F$, there exists $\delta \in \Phi^{*}$ and $h \in L=\left\langle\bigcup_{\varphi \in \Phi^{*}} \mathcal{S}^{\varphi}\right\rangle^{F}$ so that $g^{\tilde{\delta}}=g^{\delta} h$ holds. By construction, we have that $L \subseteq R$. By symmetry (as we have both $x^{\tilde{\sigma}}=x^{\sigma} r_{x}$ and $x^{\sigma}=x^{\tilde{\sigma}} r_{x}^{-1}$ ) the same arguments will show that, for each $\delta \in \Phi^{*}$ and $g \in F$, there exists $\tilde{\delta} \in \Psi^{*}$ and $h \in \tilde{L}=\left\langle\bigcup_{\varphi \in \Psi^{*}} \mathcal{S}^{\varphi}\right\rangle^{F}$ so that $g^{\delta}=g^{\tilde{\delta}} h$ holds. This would yield that each normal generator $s^{\tilde{\delta}} \in \tilde{R}$, with $s \in \mathcal{R} \cup \mathcal{S}$ and $\tilde{\delta} \in \Psi^{*}$, can be written as $s^{\tilde{\delta}}=s^{\delta} h$ for some $\delta \in \Phi^{*}$ and $h \in L \subseteq R$. In fact, $s^{\tilde{\delta}} \in \tilde{R}$ satisfies that $s^{\tilde{\delta}}=s^{\delta} h \in R$ and thus $\tilde{R} \subseteq R$. By symmetry, we would also obtain that $R \subseteq \tilde{R}$ holds. This clearly proves Proposition 3.14 . 
It therefore remains to prove that, for each $\tilde{\delta} \in \Psi^{*}$ and $g \in F$, there exists $\delta \in \Phi^{*}$ and $h \in L$ so that $g^{\tilde{\delta}}=g^{\delta} h$ holds. Each $g \in F$ is represented by a finite word $w_{g}\left(x_{i_{1}}, \ldots, x_{i_{n}}\right)$ over finitely many generators $\left\{x_{i_{1}}, \ldots, x_{i_{n}}\right\} \subseteq \mathcal{X}$. Let $\tilde{\delta} \in \Psi^{*}$ and $g \in F$ be given. We prove the assertion by induction on $m=\|\tilde{\delta}\|$. If $m=1$, then $\tilde{\delta} \in \Psi$. Moreover, we either have $\tilde{\delta}=\tilde{\sigma}$ or $\tilde{\delta} \neq \tilde{\sigma}$. If $\tilde{\delta} \neq \tilde{\sigma}$ holds, then $\tilde{\delta} \in \Phi$ and thus $g^{\tilde{\delta}}=g^{\delta} h$ for some $\delta \in \Phi$ and $h \in L$. Otherwise, if $\tilde{\delta}=\tilde{\sigma}$ holds, we obtain that

$$
g^{\tilde{\sigma}}=w_{g}\left(x_{i_{1}}, \ldots, x_{i_{n}}\right)^{\tilde{\sigma}}=w_{g}\left(x_{i_{1}}^{\tilde{\sigma}}, \ldots, x_{i_{n}}^{\tilde{\sigma}}\right)=w_{g}\left(x_{i_{1}}^{\sigma} r_{x_{i_{1}}}, \ldots, x_{i_{n}}^{\sigma} r_{x_{i_{n}}}\right) .
$$

Conjugation in the free group $F$ yields that the word $w_{g}\left(x_{i_{1}}^{\sigma} r_{x_{i_{1}}}, \ldots, x_{i_{n}}^{\sigma} r_{x_{i_{n}}}\right)$ can be written as $w_{g}\left(x_{i_{1}}^{\sigma}, \ldots, x_{i_{n}}^{\sigma}\right) \cdot h$ for some $h \in\langle\mathcal{S}\rangle^{F}$. Thus $g^{\tilde{\sigma}}=g^{\sigma} \cdot h$ for some $\sigma \in \Phi$ and $h \in\langle\mathcal{S}\rangle^{F} \subseteq L$.

For an integer $m>1$, assume that, for every $g \in F$ and $\tilde{\delta} \in \Psi^{*}$, with $\|\tilde{\delta}\|=m$, the image $g^{\tilde{\delta}} \in \tilde{R}$ satisfies that $g^{\tilde{\delta}}=g^{\delta} h$ for $\delta \in \Phi^{*}$ and some $h \in L$. Let $g \in F$ and $\tilde{\delta} \in \Psi^{*}$, with $\|\tilde{\delta}\|=m+1$, be given. Then there exist $\tilde{\omega} \in \Psi$ and $\tilde{\gamma} \in \Psi^{*}$, with $\|\tilde{\gamma}\|=n$, so that $\tilde{\delta}=\tilde{\gamma} \tilde{\omega}$ holds. By our assumption, there exist $\gamma \in \Phi^{*}$ and $h \in L$ so that $g^{\tilde{\gamma}}=g^{\gamma} h$ holds. Thus $g^{\tilde{\delta}}=g^{\tilde{\gamma} \tilde{\omega}}=\left(g^{\gamma} h\right)^{\tilde{\omega}}$. If $\tilde{\omega} \neq \tilde{\sigma}$ holds, then $\tilde{\omega} \in \Phi$ and thus $\gamma \tilde{\omega} \in \Phi^{*}$. Moreover, by construction, the normal subgroups $L=\left\langle\bigcup_{\varphi \in \Phi^{*}} \mathcal{S}^{\varphi}\right\rangle^{F}$ and $\tilde{L}=\left\langle\bigcup_{\varphi \in \Psi^{*}} \mathcal{S}^{\varphi}\right\rangle^{F}$ are $\Phi^{*}$ - and $\Psi^{*}$-invariant, respectively. Thus $h^{\tilde{\omega}} \in L$ if $\tilde{\omega} \neq \tilde{\sigma}$. Therefore, the image $g^{\tilde{\delta}}$ satisfies that $g^{\tilde{\delta}}=g^{\gamma \tilde{\omega}} h^{\tilde{\omega}}$ for some $\gamma \tilde{\omega} \in \Phi^{*}$ and $h^{\tilde{\omega}} \in L$. It suffices to consider the case $\tilde{\omega}=\tilde{\sigma}$. The elements $g^{\gamma} \in F$ and $h \in F$ are represented by finite words $w_{g^{\gamma}}\left(x_{j_{1}}, \ldots, x_{j_{n}}\right)$ and $w_{h}\left(x_{k_{1}}, \ldots, x_{k_{\ell}}\right)$, respectively. Again, conjugation in the free group $F$ yields that $w_{g^{\gamma}}\left(x_{j_{1}}, \ldots, x_{j_{n}}\right)^{\tilde{\sigma}}=w_{g^{\gamma}}\left(x_{j_{1}}^{\sigma}, \ldots, x_{j_{n}}^{\sigma}\right) u$ and $w_{h}\left(x_{k_{1}}, \ldots, x_{k_{\ell}}\right)^{\tilde{\sigma}}=w_{h}\left(x_{k_{1}}^{\sigma}, \ldots, x_{k_{\ell}}^{\sigma}\right) v$ with $u, v \in\langle\mathcal{S}\rangle^{F}$. Thus $g^{\tilde{\delta}}=g^{\tilde{\gamma} \tilde{\sigma}}=\left(g^{\gamma} h\right)^{\tilde{\sigma}}=\left(g^{\gamma \sigma} u\right)\left(h^{\sigma} v\right)$. In fact, we have that $g^{\tilde{\delta}}=g^{\gamma \sigma} h^{\prime}$ with $\gamma \sigma \in \Phi^{*}$ and $h^{\prime}=u h^{\sigma} v \in L$. Thus, for every $g \in F$ and $\tilde{\delta} \in \Psi^{*}$, the image $g^{\tilde{\delta}}$ satisfies that $g^{\tilde{\delta}}=g^{\delta} h$ with $\delta \in \Phi^{*}$ and $h \in L$. By symmetry, as we have both $x^{\tilde{\sigma}}=x^{\sigma} r_{x}$ and $x^{\sigma}=x^{\tilde{\sigma}} r_{x}^{-1}$, the same arguments will prove that for each $g \in F$ and $\delta \in \Phi^{*}$ the image $g^{\delta}$ satisfies that $g^{\delta}=g^{\tilde{\delta}} h$ with $\tilde{\delta} \in \Psi^{*}$ and $h \in \tilde{L}=\left\langle\bigcup_{\varphi \in \Psi^{*}} \mathcal{S}^{\varphi}\right\rangle^{F}$. This finishes our proof of Proposition 3.14 .

As a consequence of Proposition 3.14, we obtain the following

Corollary 3.15 Let $G=\langle\mathcal{X}|\mathcal{Q}| \Phi \mid \mathcal{R}\rangle$ be a finitely L-presented group and let $\sigma \in \Phi$ be given. Then $\sigma$ induces an endomorphism of the invariantly finitely L-presented group $H=\langle\mathcal{X}|\emptyset| \Phi \mid \mathcal{R}\rangle$. If $\psi \in \operatorname{End}(F(\mathcal{X}))$ and $\sigma$ induce the same endomorphism on $H$, then there exists a finite subset $\mathcal{S} \subseteq F(\mathcal{X})$ so that $\langle\mathcal{X}|\mathcal{Q}|(\Phi \backslash\{\sigma\}) \cup\{\psi\} \mid \mathcal{R} \cup \mathcal{S}\rangle$ is a finite L-presentation for $G$.

Proof. If $\sigma$ and $\psi$ induce the same endomorphism of $H$, there exists, for each $x \in \mathcal{X}$, an element $r_{x} \in\left\langle\bigcup_{\sigma \in \Phi^{*}} \mathcal{R}^{\sigma}\right\rangle^{F}$ with $x^{\psi}=x^{\sigma} r_{x}$. Write $\mathcal{S}=\left\{r_{x} \mid x \in \mathcal{X}\right\}$. Then Proposition 3.14 yields that $G=\langle\mathcal{X}|\mathcal{Q}|(\Phi \backslash\{\sigma\}) \cup\{\psi\} \mid \mathcal{R} \cup \mathcal{S}\rangle$.

The transformations introduced above allow us to modify a given $L$-presentation of a group. In order to prove Tietze's theorem for invariantly finitely $L$-presented groups, we choose the following set of transformations: 
Definition 3.16 An L-Tietze transformation is a transformation that

(i) adds or removes a single fixed relation (Proposition 3.4),

(ii) adds or removes a single iterated relation (Proposition 3.5),

(iii) adds or removes a single substitution (Proposition 3.12),

(iv) adds or removes a generator together with a fixed relation (Proposition 3.8),

(v) adds or removes a generator together with an iterated relation (Proposition [3.9), or that

(vi) modifies a given substitution of an L-presentation (Proposition 3.14).

\section{B.4 Applications of Tietze Transformations}

The transformations introduced in Section B.3 allow us to prove Theorem A:

Proof of Theorem A. We use similar ideas as in the proof of Tietze's theorem in [95, Chapter II]: As each $L$-Tietze transformation does not change the isomorphism type of the group, two finite $L$-presentations define isomorphic groups if one $L$-presentation can be transformed into the other by a finite sequence of $L$-Tietze transformations. In order to prove Theorem $\mathrm{A}$, it suffices to prove that two invariant finite $L$-presentations which define isomorphic groups can be transformed into each other by a finite sequence of $L$ Tietze transformations. For this purpose, we consider two invariant finite $L$-presentations $\left\langle\mathcal{X}_{1}\left|\mathcal{Q}_{1}\right| \Phi_{1} \mid \mathcal{R}_{1}\right\rangle$ and $\left\langle\mathcal{X}_{2}\left|\mathcal{Q}_{2}\right| \Phi_{2} \mid \mathcal{R}_{2}\right\rangle$ of a group $G$. By Proposition [3.7, we can assume that both $\mathcal{Q}_{1}=\emptyset$ and $\mathcal{Q}_{2}=\emptyset$ hold. We will construct an invariant finite $L$ presentation for $G$ which can be obtained from both $L$-presentations by a finite sequence of $L$-Tietze transformations. Because each $L$-Tietze transformation is reversible, this shows that we can pass from one $L$-presentation to the other by a finite sequence of $L$-Tietze transformations.

Suppose that $\mathcal{X}_{1} \cap \mathcal{X}_{2}=\emptyset$ holds. For $i \in\{1,2\}$, we denote by $F_{i}=F\left(\mathcal{X}_{i}\right)$ the free group over the alphabet $\mathcal{X}_{i}$ and by $\pi_{i}: F_{i} \rightarrow G$ the free presentation with kernel $\operatorname{ker}\left(\pi_{i}\right)=\left\langle\bigcup_{\sigma \in \Phi_{i}^{*}} \mathcal{R}_{i}^{\sigma}\right\rangle^{F_{i}}$. For each $x \in \mathcal{X}_{1}$, we choose $w_{x} \in F_{2}$ with $x^{\pi_{1}}=w_{x}^{\pi_{2}}$; i.e., the element $w_{x} \in F_{2}$ is a $\pi_{2}$-preimage of $x^{\pi_{1}} \in G$. For each $z \in \mathcal{X}_{2}$, we choose $w_{z} \in F_{1}$ with $z^{\pi_{2}}=w_{z}^{\pi_{1}}$. Define the subsets $S_{1}=\left\{x^{-1} w_{x} \mid x \in \mathcal{X}_{1}\right\}$ and $S_{2}=\left\{z^{-1} w_{z} \mid z \in \mathcal{X}_{2}\right\}$ of the free group $F=F\left(\mathcal{X}_{1} \cup \mathcal{X}_{2}\right)$ over the alphabet $\mathcal{X}_{1} \cup \mathcal{X}_{2}$. By Proposition 3.9. we can add the finitely many generators $z \in \mathcal{X}_{2}$ together with the iterated relation $z^{-1} w_{z} \in S_{2}$ if we extend each substitution $\sigma \in \Phi_{1}$ to the free group $F$ by

$$
\tilde{\sigma}:\left\{\begin{aligned}
x & \mapsto x^{\sigma}, \quad \text { for each } x \in \mathcal{X}_{1} \\
z & \mapsto w_{z}^{\sigma}, \quad \text { for each } z \in \mathcal{X}_{2}
\end{aligned}\right.
$$

This yields the finite $L$-presentation

$$
\left\langle\mathcal{X}_{1} \cup \mathcal{X}_{2}|\emptyset|\{\tilde{\sigma}\}_{\sigma \in \Phi_{1}} \mid \mathcal{R}_{1} \cup\left\{z^{-1} w_{z}\right\}_{z \in \mathcal{X}_{2}}\right\rangle
$$


for the group $G$. The natural homomorphisms $\pi_{1}: F_{1} \rightarrow G$ and $\pi_{2}: F_{2} \rightarrow G$ extend to a natural homomorphism $\pi: F \rightarrow G$ that is induced by the map

$$
\pi:\left\{\begin{aligned}
x & \mapsto x^{\pi_{1}}, \quad \text { for each } x \in \mathcal{X}_{1}, \\
z & \mapsto z^{\pi_{2}}, \text { for each } z \in \mathcal{X}_{2}
\end{aligned}\right.
$$

Its kernel satisfies $\operatorname{ker}(\pi)=\left\langle\bigcup_{\sigma \in \Phi_{1}^{*}}\left(\mathcal{R}_{1} \cup S_{2}\right)^{\tilde{\sigma}}\right\rangle^{F}$. For $x \in \mathcal{X}_{1}$ and $x^{-1} w_{x} \in S_{1}$, we have $x^{\pi}=x^{\pi_{1}}=w_{x}^{\pi_{2}}=w_{x}^{\pi}$ and thus $x^{-1} w_{x} \in \operatorname{ker}(\pi)$ holds. For each $r \in \mathcal{R}_{2}$, we have $r^{\pi}=r^{\pi_{2}}=1$ and thus $r \in \operatorname{ker}(\pi)$ holds. Since the kernel $\operatorname{ker}(\pi)$ is $\left\{\tilde{\sigma} \mid \sigma \in \Phi_{1}\right\}^{*}$ invariant, by construction, Proposition 3.5 yields that

$$
G \cong\left\langle\mathcal{X}_{1} \cup \mathcal{X}_{2}|\emptyset|\{\tilde{\sigma}\}_{\sigma \in \Phi_{1}} \mid \mathcal{R}_{1} \cup \mathcal{R}_{2} \cup S_{1} \cup S_{2}\right\rangle .
$$

As the invariant finite $L$-presentations $\left\langle\mathcal{X}_{1}|\emptyset| \Phi_{1} \mid \mathcal{R}_{1}\right\rangle$ and $\left\langle\mathcal{X}_{2}|\emptyset| \Phi_{2} \mid \mathcal{R}_{2}\right\rangle$ define isomorphic groups and every $\psi \in \Phi_{2}$ induces an endomorphism of the whole group, we can extend $\psi$ to an endomorphism of the free group $F$ over the alphabet $\mathcal{X}_{1} \cup \mathcal{X}_{2}$ that induces the same endomorphism on $G$ as $\psi$ does. More precisely, for each $\psi \in \Phi_{2}$, we define an endomorphism of the free group $F$ that is induced by the map

$$
\tilde{\psi}:\left\{\begin{aligned}
z & \mapsto z^{\psi}, \quad \text { for each } z \in \mathcal{X}_{2} \\
x & \mapsto w_{x}^{\psi},
\end{aligned}\right.
$$

By construction, the normal subgroup $\left\langle\bigcup_{\sigma \in \Phi_{1}^{*}}\left(\mathcal{R}_{1} \cup \mathcal{R}_{2} \cup S_{1} \cup S_{2}\right)^{\tilde{\sigma}}\right\rangle^{F}$ is $\tilde{\psi}$-invariant. Thus, by Proposition 3.12, the group $G$ satisfies that

$$
G \cong\left\langle\mathcal{X}_{1} \cup \mathcal{X}_{2}|\emptyset|\{\tilde{\sigma}\}_{\sigma \in \Phi_{1}} \cup\{\tilde{\psi}\}_{\psi \in \Phi_{2}} \mid \mathcal{R}_{1} \cup \mathcal{R}_{2} \cup S_{1} \cup S_{2}\right\rangle .
$$

Since the $L$-presentations $\left\langle\mathcal{X}_{1}\left|\mathcal{Q}_{1}\right| \Phi_{1} \mid \mathcal{R}_{1}\right\rangle$ and $\left\langle\mathcal{X}_{2}\left|\mathcal{Q}_{2}\right| \Phi_{2} \mid \mathcal{R}_{2}\right\rangle$ were finite, we have applied only finitely many $L$-Tietze transformations from Definition 3.16. Therefore, starting with the $L$-presentation $\left\langle\mathcal{X}_{1}\left|\mathcal{Q}_{1}\right| \Phi_{1} \mid \mathcal{R}_{1}\right\rangle$ we have obtained the $L$-presentation in Eq. (B.7) after finitely many steps. By symmetry, though, we would also obtain the finite $L$-presentation in Eq. (B.7) if we would have started with the finite $L$-presentation $\left\langle\mathcal{X}_{2}\left|\mathcal{Q}_{2}\right| \Phi_{2} \mid \mathcal{R}_{2}\right\rangle$. Since each $L$-Tietze transformation is reversible, we can therefore transform the finite $L$-presentation in Eq. (B.7) to the finite $L$-presentation $\left\langle\mathcal{X}_{2}\left|\mathcal{Q}_{2}\right| \Phi_{2}\right|$ $\left.\mathcal{R}_{2}\right\rangle$. This yields a finite sequence of $L$-Tietze transformations that allows us to transform the $L$-presentation $\left\langle\mathcal{X}_{1}\left|\mathcal{Q}_{1}\right| \Phi_{1} \mid \mathcal{R}_{1}\right\rangle$ to the $L$-presentation $\left\langle\mathcal{X}_{2}\left|\mathcal{Q}_{2}\right| \Phi_{2} \mid \mathcal{R}_{2}\right\rangle$ and vice versa.

Similarly, the Tietze transformations in Section B.3 also allow us to prove that two arbitrary finite $L$-presentations could be transformed into each other by a finite sequence of Tietze transformations.

Another application of $L$-Tietze transformations is to prove that 'being invariantly finitely $L$-presented' is an abstract property of a group that does not depend on the generating set of the group; that is, if a group admits an invariant finite $L$-presentation with respect to one finite generating set, then so it does with respect to any other finite 
generating set. This result was already posed in [6, Proposition 2.2]. However, its proof contains a gap: Consider the invariant finite $L$-presentation

$$
\left.\mathfrak{G} \cong\left\langle\{a, b, c, d\}\left|\left\{a^{2}, b^{2}, c^{2}, d^{2}, b c d\right\}\right|\{\sigma\}\right|\left\{(a d)^{4},(\text { adacac })^{4}\right\}\right\rangle
$$

from Theorem 2.2, where $\sigma$ is induced by the map $a \mapsto a c a, b \mapsto d, c \mapsto b$, and $d \mapsto c$. Then $\sigma$ is a monomorphism of the free group $F=F(\{a, b, c, d\})$. The transformations in the proof of [6, Proposition 2.2] keep the rank of im $(\sigma)$ constant and therefore, they do not allow to prove that the Grigorchuk group admits an invariant finite $L$-presentation with generators $\{a, c, d\}$ as in Eq. (B.2). The $L$-Tietze transformations from Section B.3 allow us to address this gap:

Proof of Theorem $\mathbb{B}$, Let $\mathcal{Y}=\left\{y_{1}, \ldots, y_{n}\right\}$ be an arbitrary finite generating set of the invariantly finitely $L$-presented group $G=\langle\mathcal{X}|\mathcal{Q}| \Phi \mid \mathcal{R}\rangle$. As $G$ is invariantly $L$ presented, we can assume that $\mathcal{Q}=\emptyset$ holds. Since $\mathcal{Y}$ generates $G$, there exists, for each $x \in \mathcal{X}$, a word $w_{x}\left(y_{1}, \ldots, y_{n}\right)$ over the generators $\mathcal{Y}$ so that $x=_{G} w_{x}\left(y_{1}, \ldots, y_{n}\right)$ holds. Since $\mathcal{X}=\left\{x_{1}, \ldots, x_{m}\right\}$ also generates $G$, there exists, for each $y \in \mathcal{Y}$, a word $w_{y}\left(x_{1}, \ldots, x_{m}\right)$ so that $y={ }_{G} w_{y}\left(x_{1}, \ldots, x_{m}\right)$ holds. Suppose that $\mathcal{X} \cap \mathcal{Y}=\emptyset$ holds. For each $\sigma \in \Phi$, define an endomorphism $\tilde{\sigma}$ of the free group $E$ over the alphabet $\mathcal{X} \cup \mathcal{Y}$ that is induced by the map

$$
\tilde{\sigma}:\left\{\begin{array}{lll}
x & \mapsto x^{\sigma}, & \text { for each } x \in \mathcal{X} \\
y & \mapsto w_{y}\left(x_{1}, \ldots, x_{m}\right)^{\sigma}, & \text { for each } y \in \mathcal{Y} .
\end{array}\right.
$$

Then, by Proposition 3.9, a finite $L$-presentation for the group $G$ is given by

$$
\left\langle\mathcal{X} \cup \mathcal{Y}|\emptyset|\{\tilde{\sigma}\}_{\sigma \in \Phi} \mid \mathcal{R} \cup\left\{y^{-1} w_{y}\left(x_{1}, \ldots, x_{m}\right)\right\}_{y \in \mathcal{Y}}\right\rangle .
$$

As this $L$-presentation is invariant, every $\tilde{\sigma}$, with $\sigma \in \Phi$, induces an endomorphism of the group $G$. Thus, as $x={ }_{G} w_{x}\left(y_{1}, \ldots, y_{n}\right)$ holds, we have $x^{\tilde{\sigma}}={ }_{G} w_{x}\left(y_{1}, \ldots, y_{n}\right)^{\tilde{\sigma}}$ for each $\sigma \in \Phi^{*}$. By Proposition 3.5, we have that

$$
G \cong\left\langle\mathcal{X} \cup \mathcal{Y}|\emptyset|\{\tilde{\sigma}\}_{\sigma \in \Phi} \mid \mathcal{R} \cup\left\{y^{-1} w_{y}\right\}_{y \in \mathcal{Y}} \cup\left\{x^{-1} w_{x}\right\}_{x \in \mathcal{X}}\right\rangle
$$

Since $\mathcal{Y}$ generates $H$, for each $z \in \mathcal{X} \cup \mathcal{Y}$ and $\sigma \in \Phi$, the image $z^{\tilde{\sigma}}$ is represented by a word $v_{z, \sigma}\left(y_{1}, \ldots, y_{n}\right)$ over the generators $\mathcal{Y}$ so that $z^{\tilde{\sigma}}={ }_{G} v_{z, \sigma}\left(y_{1}, \ldots, y_{n}\right)$ holds. Since the $L$-presentation in Eq. (B.8) is invariant, Proposition 3.14 applies to the relation $r=\left(z^{\tilde{\sigma}}\right)^{-1} v_{z, \sigma}\left(y_{1}, \ldots, y_{n}\right)$ and it shows that $G$ admits the following finite $L$-presentation

$$
\left\langle\mathcal{X} \cup \mathcal{Y}|\emptyset|\{\widehat{\sigma}\}_{\sigma \in \Phi} \mid \mathcal{R} \cup\left\{x^{-1} w_{x}\right\}_{x \in \mathcal{X}} \cup\left\{y^{-1} w_{y}\right\}_{y \in \mathcal{Y}} \cup\left\{\left(z^{\tilde{\sigma}}\right)^{-1} v_{z, \sigma}\right\}_{z \in \mathcal{X} \cup \mathcal{Y}, \sigma \in \Phi}\right\rangle
$$

where the substitutions $\widehat{\sigma}$ are induced by the maps

$$
\widehat{\sigma}: z \mapsto v_{z, \sigma}\left(y_{1}, \ldots, y_{n}\right), \text { for each } z \in \mathcal{X} \cup \mathcal{Y} .
$$

We use the iterated relations $x^{-1} w_{x}\left(y_{1}, \ldots, y_{n}\right)$, with $x \in \mathcal{X}$, to replace every occurrence of $x \in \mathcal{X}$ among the iterated relations

$$
\mathcal{R} \cup\left\{y^{-1} w_{y}\left(x_{1}, \ldots, x_{m}\right)\right\}_{y \in \mathcal{Y}} \cup\left\{\left(z^{\tilde{\sigma}}\right)^{-1} v_{z, \sigma}\left(y_{1}, \ldots, y_{n}\right)\right\}_{z \in \mathcal{X} \cup \mathcal{Y}, \sigma \in \Phi}
$$


by $w_{x}\left(y_{1}, \ldots, y_{n}\right)$. This yields a finite set of relations $\tilde{\mathcal{S}}$ that can be considered as a finite subset of the free group over the alphabet $\mathcal{Y}$. Replacing the relations in Eq. (B.9) by $\tilde{\mathcal{S}}$ does not change the isomorphism type of the group. The group $G$ satisfies that $G \cong\left\langle\mathcal{X} \cup \mathcal{Y}|\emptyset|\{\hat{\sigma} \mid \sigma \in \Phi\} \mid \tilde{\mathcal{S}} \cup\left\{x^{-1} w_{x} \mid x \in \mathcal{X}\right\}\right\rangle$. By Proposition 3.9. the group $G$ is invariantly finitely $L$-presented by $\left\langle\mathcal{Y}|\emptyset|\{\hat{\sigma}\}_{\sigma \in \Phi} \mid \tilde{\mathcal{S}}\right\rangle$.

\section{B.5 Finitely generated normal subgroups of finitely pre- sented groups}

In this section, we consider finitely generated normal subgroups of finitely presented groups. By Higman's embedding theorem [81, every finitely generated group embeds into a finitely presented group if and only if it is recursively presented. This theorem classifies the finitely generated subgroups of a finitely presented group. The normal subgroups of a finitely presented group are invariantly $L$-presented:

Proposition 5.17 Every normal subgroup of a finitely presented group admits an invariant L-presentation whose substitutions induce automorphisms of the subgroup. If the normal subgroup has finite index, it is invariantly finitely L-presented.

Proof. This follows from the proof of [70, Theorem 6.1]; cf. Lemma 5.19 below.

The $L$-presentation in Lemma 5.19 below is an ascending $L$-presentation with finitely many substitutions and finitely many iterated relations. It has finitely many generators if and only if the subgroup has finite index. The substitutions of this $L$-presentation induce automorphisms of the subgroup since they are induced by conjugation in the finitely presented group.

On the other hand, as every finite $L$-presentation is recursive, finitely $L$-presented groups embed into finitely presented groups. As indicated in [22], a finitely $L$-presented group embeds as a normal subgroup into a finitely presented group if we assume that every substitution of the $L$-presentation induces an automorphism of the subgroup:

Proposition 5.18 Every group that admits an invariant finite L-presentation, whose substitutions induce automorphisms of the group, embeds as a normal subgroup into a finitely presented group.

Proof. If $H=\left\langle\mathcal{Z}|\emptyset|\left\{\delta_{1}, \ldots, \delta_{n}\right\} \mid \mathcal{R}\right\rangle$ is invariantly finitely $L$-presented so that each $\delta_{i}$ induces an automorphism of $H$, the base group $H$ embeds into the HNN-extension $G_{1}$ relative to the isomorphism $\delta_{1}: H \rightarrow H$ which is induced by the substitution $\delta_{1}$. The HNN-extension $G_{1}$ is given by the presentation $G_{1}=\left\langle\mathcal{Z} \cup\left\{t_{1}\right\}\right| \bigcup_{\sigma \in \Phi^{*}} \mathcal{R}^{\sigma} \cup\left\{t_{1}^{-1} z t_{1}=\right.$ $\left.\left.z^{\delta_{1}} \mid z \in \mathcal{Z}\right\}\right\rangle$ where $\Phi=\left\{\delta_{1}, \ldots, \delta_{n}\right\}$. Denote by $H_{1}$ the image of $H$ in $G_{1}$. Then $\delta_{2}$ induces an automorphism of the subgroup $H_{1} \leq G_{1}$. Thus we can form the HNNextension $G_{2}$ relative to the isomorphism $\delta_{2}: H_{1} \rightarrow H_{1}$. As the base group $G_{1}$ embeds into the HNN-extension $G_{2}$, the subgroup $H_{1}$ embeds into $G_{2}$ as well. Iterating this process, we obtain a group $G_{n}=\left\langle\mathcal{Z} \cup\left\{t_{1}, \ldots, t_{n}\right\}\right| \bigcup_{\sigma \in \Phi^{*}} \mathcal{R}^{\sigma} \cup\left\{t_{i}^{-1} z t_{i}=z^{\delta_{i}} \mid 1 \leq i \leq\right.$ $n\}\rangle$ in which $H$ embeds. Tietze transformations that replace every $\delta_{i}$-image $z^{\delta_{i}}$ by the 
conjugate $t_{i}^{-1} z t_{i}$ in the relations $\bigcup_{\sigma \in \Phi^{*}} \mathcal{R}^{\sigma}$ eventually show that $G_{n}=\left\langle\mathcal{Z} \cup\left\{t_{1}, \ldots, t_{n}\right\}\right|$ $\left.\mathcal{R} \cup\left\{t_{i}^{-1} z t_{i}=z^{\delta_{i}} \mid 1 \leq i \leq n, z \in \mathcal{Z}\right\}\right\rangle$ is finitely presented. The invariantly finitely $L$ presented group $H$ embeds into this finitely presented group by identifying the generator in $\mathcal{Z}$. The image of $H$ in $G_{n}$ is obviously a normal subgroup of $G_{n}$.

In the following, we use the constructions from [22] to prove Theorem [C. Since every normal subgroup of a finitely presented group admits an invariant $L$-presentation with finitely many substitutions and finitely many iterated relations, it suffices to show that the $L$-presentation in Lemma 5.19 below could be transformed into an invariant finite $L$-presentation. For this purpose, though, we need to eliminate (possibly) infinitely many generators from the $L$-presentation and we need to modify finitely many substitutions. However, Proposition 3.14 adds iterated relations for each modification of a substitution. Hence, we need to ensure that this process still gives a finite $L$-presentation. In the following, we generalize the constructions from [22]:

\section{B.5.1 Preliminaries}

Let $G$ be a finitely presented group and let $H \unlhd G$ be a finitely generated normal subgroup. Then $G / H$ is finitely presented. Moreover, if $H=\left\langle a_{1}, \ldots, a_{m}\right\rangle$ and $G / H=$ $\left\langle s_{1} H, \ldots, s_{n} H\right\rangle$ hold, there exists a finite presentation $\left\langle\left\{a_{1}, \ldots, a_{m}, s_{1}, \ldots, s_{n}\right\} \mid \mathcal{R}\right\rangle$ for $G$. The proof of [70, Theorem 6.1] yields the following

Lemma 5.19 Let $\left\langle\left\{a_{1}, \ldots, a_{m}, s_{1}, \ldots, s_{n}\right\} \mid \mathcal{R}\right\rangle$ be a finite presentation for $G$ and write $\mathcal{S}=\left\{s_{1}^{ \pm 1}, \ldots, s_{n}^{ \pm 1}\right\}$. If $\mathcal{T}$ is a Schreier transversal for $H=\left\langle a_{1}, \ldots, a_{m}\right\rangle$ in $G$ and $\mathcal{Y}$ are the Schreier generators of $H$, then $H$ is invariantly L-presented by

$$
\left\langle\mathcal{Y}|\emptyset|\left\{\delta_{x} \mid x \in \mathcal{S}\right\} \mid \mathcal{R}^{\tau}\right\rangle
$$

where $\delta_{x}$ denotes the endomorphism of the free group $F(\mathcal{Y})$ that is induced by conjugation with $x \in \mathcal{S}$ and $\tau$ denotes the Reidemeister-rewriting.

Proof. This follows from the Reidemeister-Schreier theorem, see [95, Section II.4] and the proof of [70, Theorem 6.1]. Clearly, one can always omit the endomorphisms $\delta_{x}$ with $x \in\left\{a_{1}, \ldots, a_{m}\right\}$ as they give inner automorphisms of the subgroup $H$.

Since $\mathcal{S}$ and $\mathcal{R}$ are finite, the $L$-presentation in Lemma 5.19 is finite if and only if $H$ has finite index in $G$; in this case $\mathcal{Y}$ is finite. Finite index subgroups of finitely $L$-presented groups have been studied in [70]. It was shown that each normal subgroup of a finitely presented group with finite index is invariantly finitely $L$-presented. In the following, we therefore assume that $[G: H]=\infty$ holds.

The strategy in the proof of Theorem $[$ will be as follows: Our choice of the generating set of the finitely presented group allows us to assume that $H$ 's generators $\mathcal{Z}=\left\{a_{1}, \ldots, a_{m}\right\}$ are Schreier generators of $H$. We therefore obtain an embedding $\chi: F(\mathcal{Z}) \rightarrow F(\mathcal{Y})$ and we will construct an epimorphism $\gamma: F(\mathcal{Y}) \rightarrow F(\mathcal{Z})$ so that the free presentation $\pi: F(\mathcal{Y}) \rightarrow H$ that is given by the $L$-presentation in Lemma 5.19 satisfies $\gamma \chi \pi=\pi$. Since the $L$-presentation in Lemma 5.19 is invariant, there exists, for 
each $\sigma \in \Phi=\left\{\delta_{x} \mid x \in \mathcal{S}\right\}$, an endomorphism $\widehat{\sigma} \in \operatorname{End}(H)$ so that $\sigma \pi=\pi \widehat{\sigma}$ holds. In general, we cannot assume that there also exists an endomorphism $\tilde{\sigma} \in \operatorname{End}(F(\mathcal{Z})$ ) so that $\sigma \gamma=\gamma \tilde{\sigma}$ holds. Therefore, we will construct a normal subgroup $N \unlhd F(\mathcal{Z})$ so that $\psi: F(\mathcal{Z}) \rightarrow F(\mathcal{Z}) / N, g \mapsto g N$ yields the existence of $\bar{\sigma} \in \operatorname{End}(F(\mathcal{Z}) / N)$ with $\sigma \gamma \psi=\gamma \psi \bar{\sigma}$. These constructions will give the following commutative diagram:

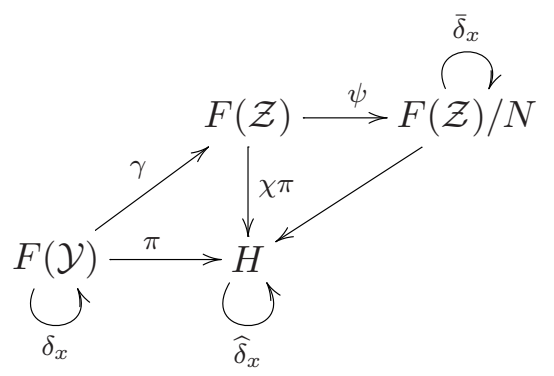

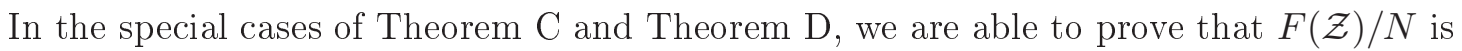
invariantly finitely $L$-presented and so is the subgroup $H$. The normal $\operatorname{subgroup~} N$ will be generated, as a normal subgroup, by the iterated relations that Proposition 3.14 adds when modifying the substitutions of the $L$-presentation in Lemma 5.19, These relations were omitted in [22]. It is not clear whether or not these relations are necessary to define the subgroup $H$.

In the remainder of this section, we generalize the constructions from 22] to obtain the commutative diagram above. The generating set $\mathcal{X}=\left\{a_{1}, \ldots, a_{m}, s_{1}, \ldots, s_{n}\right\}$ of the finitely presented group $G$ yields that the generators $\mathcal{Z}=\left\{a_{1}, \ldots, a_{m}\right\}$ are Schreier generators of $H$. Hence, there exists a natural embedding $\chi: F(\mathcal{Z}) \rightarrow F(\mathcal{Y})$ which is induced by embedding the generators $\mathcal{Z}$ into $\mathcal{Y}$. It suffices to remove the Schreier generators $\mathcal{Y} \backslash \mathcal{Z}$ from the invariant $L$-presentation in Lemma 5.19. Since $H$ is generated by $\mathcal{Z}=\left\{a_{1}, \ldots, a_{m}\right\}$, every $y \in \mathcal{Y}$ can be represented, as an element of $H$, by a word over $\mathcal{Z}$. This yields an epimorphism $\gamma: F(\mathcal{Y}) \rightarrow F(\mathcal{Z})$ which maps every $y \in \mathcal{Y}$ to a word $y^{\gamma} \in F(\mathcal{Z})$ over the alphabet $\mathcal{Z}$ that represents the same element in $H$; i.e., we have

$$
\left\{y^{-1} y^{\gamma \chi} \mid y \in \mathcal{Y} \backslash \mathcal{Z}\right\} \subseteq \operatorname{ker}(\pi),
$$

where $\pi: F(\mathcal{Y}) \rightarrow H$ denotes the free presentation from Lemma 5.19. Note that Eq. (B.10) yields that $\iota=\chi \pi$ defines an epimorphism $\iota: F(\mathcal{Z}) \rightarrow H$ with $\gamma \iota=\pi$. The following lemma generalizes [22, Lemma 4].

Lemma 5.20 If $H \cong\langle\mathcal{Y} \mid \mathcal{S}\rangle$ and $\gamma: F(\mathcal{Y}) \rightarrow F(\mathcal{Z})$ is an epimorphism so that

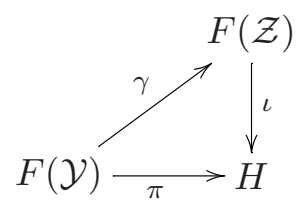

commutes, $\left\langle\mathcal{Z} \mid \mathcal{S}^{\gamma}\right\rangle$ is a presentation for $H$. 
Proof. Since $\pi=\gamma \iota$ is onto, it suffices to prove that $\operatorname{ker}(\iota)=\left\langle\mathcal{S}^{\gamma}\right\rangle^{F(\mathcal{Z})}$ holds. For $r \in \mathcal{S}$, we have that $r^{\gamma \iota}=r^{\pi}=1$ and so $r^{\gamma} \in \operatorname{ker}(\iota)$. Thus $\left\langle\mathcal{S}^{\gamma}\right\rangle^{F(\mathcal{Z})} \subseteq \operatorname{ker}(\iota)$. If $g \in \operatorname{ker}(\iota)$ holds, there exists $h \in F(\mathcal{Y})$ with $h^{\gamma}=g$ as $\gamma$ is surjective. Then $h^{\pi}=h^{\gamma \iota}=g^{\iota}=1$ and $h \in \operatorname{ker}(\pi)=\langle\mathcal{S}\rangle^{F(\mathcal{Z})}$. Thus $g=h^{\gamma} \in\left\langle\mathcal{S}^{\gamma}\right\rangle^{F(\mathcal{Z})}$.

Thus, by Lemma 5.19 and Lemma 5.20, the subgroup $H$ has a presentation of the form

$$
H=\left\langle\mathcal{Z} \mid\left\{\left(r^{\tau \sigma}\right)^{\gamma} \mid r \in \mathcal{R}, \sigma \in \Phi^{*}\right\}\right\rangle
$$

where $\Phi=\left\{\delta_{x} \mid x \in \mathcal{S}\right\}$ and $\tau$ denotes the Reidemeister rewriting. This presentation can be considered as a finite $L$-presentation if, for each $\sigma \in \Phi$, there exists an endomorphism $\tilde{\sigma} \in \operatorname{End}(F(\mathcal{Z}))$ with $\sigma \gamma=\gamma \tilde{\sigma}$. The following lemma yields the existence of such endomorphisms $\tilde{\sigma} \in \operatorname{End}(F(\mathcal{Z}))$ :

Lemma 5.21 For groups $L$ and $M$, an epimorphism $\pi: L \rightarrow M$, and an endomorphism $\delta \in \operatorname{End}(L)$, there exists a (unique) endomorphism $\Delta \in \operatorname{End}(M)$ with $\delta \pi=\pi \Delta$ if and only if $\operatorname{ker}(\pi)^{\delta} \subseteq \operatorname{ker}(\pi)$ holds.

Proof. The proof is straightforward.

Therefore, if the kernel $\operatorname{ker}(\gamma)$ is $\sigma$-invariant, for each $\sigma \in \Phi$, the subgroup $H$ would be invariantly finitely $L$-presented by $\left\langle\mathcal{Z}|\emptyset|\left\{\tilde{\delta}_{x} \mid \delta_{x} \in \Phi\right\} \mid \mathcal{R}^{\tau \gamma}\right\rangle$. In general, though, we cannot assume that each $\sigma \in \Phi$ leaves the kernel $\operatorname{ker}(\gamma)$ invariant. If we consider the natural embedding $\chi: F(\mathcal{Z}) \rightarrow F(\mathcal{Y})$ that is induced by embedding the generators $\mathcal{Z}$ into $\mathcal{Y}$, the kernel $\operatorname{ker}(\gamma)$ satisfies

Lemma 5.22 If $\chi: F(\mathcal{Z}) \rightarrow F(\mathcal{Y})$ is an embedding with $\left.\gamma \chi\right|_{\mathcal{Z}}=\operatorname{id}_{\mathcal{Z}}$, then $\chi \gamma=\operatorname{id}_{F(\mathcal{Z})}$ and $\operatorname{ker}(\gamma)=\left\langle\left\{y^{-1} y^{\gamma \chi} \mid y \in \mathcal{Y} \backslash \mathcal{Z}\right\}\right\rangle^{F(\mathcal{Y})}$ hold.

Proof. Since $\left.\gamma \chi\right|_{\mathcal{Z}}=\mathrm{id}_{\mathcal{Z}}$ holds, the map $\gamma \chi$ induces the identity on the free subgroup $E=\langle\mathcal{Z}\rangle \leq F(\mathcal{Y})$. For $g \in F(\mathcal{Z})$, we have $g^{\chi} \in E$ and $g^{\chi \gamma \chi}=g^{\chi}$. Thus $\left(g^{-1} g^{\chi \gamma}\right)^{\chi}=1$ and, as $\chi$ is injective, we have $g^{-1} g^{\chi \gamma}=1$ or

$$
\chi \gamma=\operatorname{id}_{F(\mathcal{Z})} .
$$

For each $y \in \mathcal{Y} \backslash \mathcal{Z}$, we have that $\left(y^{-1} y^{\gamma \chi}\right)^{\gamma}=y^{-\gamma} y^{\gamma \chi \gamma}=y^{-\gamma} y^{\gamma}=1$. Therefore $N=\left\langle\left\{y^{-1} y^{\gamma \chi} \mid y \in \mathcal{Y} \backslash \mathcal{Z}\right\}\right\rangle^{F(\mathcal{Y})}$ satisfies that $N \subseteq \operatorname{ker}(\gamma)$. Let $g \in \operatorname{ker}(\gamma)$ be given. Then $g \in F(\mathcal{Y})$ is represented by a finite word $w\left(y_{i_{1}}, \ldots, y_{i_{n}}, a_{1}, \ldots, a_{m}\right)$ with $\left\{y_{i_{1}}, \ldots, y_{i_{n}}\right\} \subseteq$ $\mathcal{Y} \backslash \mathcal{Z}$. Modulo the normal subgroup $N$, we can replace every occurrence of $y \in \mathcal{Y} \backslash \mathcal{Z}$ by $y^{\gamma \chi} \in E$; i.e., we have $g=w\left(y_{i_{1}}, \ldots, y_{i_{n}}, a_{1}, \ldots, a_{m}\right)=w\left(y_{i_{1}}^{\gamma \chi}, \ldots, y_{i_{n}}^{\gamma \chi}, a_{1}, \ldots, a_{m}\right) \cdot h$ for some $h \in N$. As $g \in \operatorname{ker}(\gamma)$ and $h \in N \subseteq \operatorname{ker}(\gamma)$ hold, we have

$$
1=g^{\gamma \chi}=w\left(y_{i_{1}}^{\gamma \chi \gamma \chi}, \ldots, y_{i_{n}}^{\gamma \chi \gamma \chi}, a_{1}^{\gamma \chi}, \ldots, a_{m}^{\gamma \chi}\right) \cdot h^{\gamma \chi}=w\left(y_{i_{1}}^{\gamma \chi}, \ldots, y_{i_{n}}^{\gamma \chi}, a_{1}, \ldots, a_{m}\right) \cdot 1 .
$$

Similarly, modulo the normal subgroup $N$, we can replace every occurrence of $y^{\gamma \chi}$ by $y$. There exists $k \in N$ with $1=w\left(y_{i_{1}}^{\gamma \chi}, \ldots, y_{i_{n}}^{\gamma \chi}, a_{1}, \ldots, a_{m}\right)=w\left(y_{i_{1}}, \ldots, y_{i_{n}}, a_{1}, \ldots, a_{m}\right) \cdot k=$ $g \cdot k$. Thus $g \in N$ and $N=\operatorname{ker}(\gamma)$. 
Even though $\delta_{x} \in \Phi$ may not translate directly to $\tilde{\delta}_{x} \in \operatorname{End}(F(\mathcal{Z}))$, there exists a normal subgroup $N \unlhd F(\mathcal{Z})$ and a homomorphism $\psi: F(\mathcal{Z}) \rightarrow F(\mathcal{Z}) / N, g \mapsto g N$ so that $\operatorname{ker}(\gamma \psi)^{\delta_{x}} \subseteq \operatorname{ker}(\gamma \psi)$ holds: For each $\delta_{x} \in \Phi$, define $\tilde{\delta}_{x}=\chi \delta_{x} \gamma \in \operatorname{End}(F(\mathcal{Z}))$. Consider the normal subgroup

$$
N=\left\langle\bigcup_{\sigma \in \tilde{\Phi}^{*}}\left(\left\{\left(y^{-1} y^{\gamma \chi}\right)^{\delta_{x} \gamma}\right\}_{y \in \mathcal{Y} \backslash \mathcal{Z}, x \in \mathcal{S}}\right)^{\sigma}\right\rangle^{F(\mathcal{Z})}
$$

where $\tilde{\Phi}=\left\{\tilde{\delta}_{x} \mid \delta_{x} \in \Phi\right\}$. By construction, $N$ satisfies $N^{\tilde{\delta}_{x}} \subseteq N$ and thus there exists a unique endomorphism $\bar{\delta}_{x}: F(\mathcal{Z}) / N \rightarrow F(\mathcal{Z}) / N, g N \mapsto g^{\tilde{\delta}_{x}} N$ with $\tilde{\delta}_{x} \psi=\psi \bar{\delta}_{x}$. The normal subgroup $N$ allows us to translate $\delta_{x} \in \Phi$ to $\bar{\delta}_{x} \in \operatorname{End}(F(\mathcal{Z}) / N)$ with $\delta_{x} \gamma \psi=\gamma \psi \bar{\delta}_{x}$ :

Lemma 5.23 For each $x \in \mathcal{S}$, we have that $\operatorname{ker}(\gamma \psi)^{\delta_{x}} \subseteq \operatorname{ker}(\gamma \psi)$.

Proof. The kernel $\operatorname{ker}(\gamma \psi)=\operatorname{ker}(\gamma) N^{\gamma^{-1}}$ satisfies that

$$
\operatorname{ker}(\gamma \psi)=\left\langle\left\{y^{-1} y^{\gamma \chi}\right\}_{y \in \mathcal{Y} \backslash \mathcal{Z}} \cup \bigcup_{\tilde{\sigma} \in \Phi^{*}}\left\{\left(y^{-1} y^{\gamma \chi}\right)^{\delta_{z} \gamma \tilde{\sigma} \chi}\right\}_{\substack{y \in \mathcal{Y} \backslash \mathcal{Z} \\ z \in \mathcal{S}}}\right\rangle^{F(\mathcal{Y})}
$$

The generator $\left(y^{-1} y^{\gamma \chi}\right)^{\delta_{z} \gamma \tilde{\sigma} \chi}$ is mapped by $\delta_{x} \gamma$ to $\left(y^{-1} y^{\gamma \chi}\right)^{\delta_{z} \gamma \tilde{\sigma} \chi \delta_{x} \gamma}=\left(y^{-1} y^{\gamma \chi}\right)^{\delta_{z} \gamma \tilde{\sigma} \tilde{\delta}_{x}} \in N$ while $y^{-1} y^{\gamma \chi}$ is mapped to $\left(y^{-1} y^{\gamma \chi}\right)^{\delta_{x} \gamma} \in N$.

The endomorphisms $\delta_{x} \in \operatorname{End}(F(\mathcal{Y})), \tilde{\delta}_{x} \in \operatorname{End}(F(\mathcal{Z}))$, and $\bar{\delta}_{x} \in \operatorname{End}(F(\mathcal{Z}) / N)$ also satisfy that

$$
\tilde{\delta}_{x} \psi=\chi \delta_{x} \gamma \psi=\chi \gamma \psi \bar{\delta}_{x}=\psi \bar{\delta}_{x}
$$

Since the $L$-presentation in Lemma 5.19 is invariant, there exists $\widehat{\delta}_{x} \in \operatorname{End}(H)$ with $\delta_{x} \pi=\pi \widehat{\delta}_{x}$. The subgroup $H$ is a homomorphic image of $F(\mathcal{Z}) / N$ :

Lemma 5.24 Let $\iota: F(\mathcal{Z}) \rightarrow H, g \mapsto g^{\chi \pi}$ be given. Then $\gamma \iota=\pi$ and $N \leq \operatorname{ker}(\iota)$.

Proof. The first assertion follows from the definition of $\gamma$ in Eq. (B.10) above. For $\delta_{x} \in \Phi$, we have $\tilde{\delta}_{x} \iota=\chi \delta_{x} \gamma \iota=\chi \delta_{x} \pi=\chi \pi \widehat{\delta}_{x}=\iota \widehat{\delta}_{x}$ and $\gamma \chi \pi=\gamma \iota=\pi$. Thus $\left(y^{-1} y^{\gamma \chi}\right)^{\pi}=y^{-\pi} y^{\gamma \chi \pi}=y^{-\pi} y^{\pi}=1$. For $\tilde{\sigma} \in \tilde{\Phi}^{*}$ with $\tilde{\sigma}=\tilde{\delta}_{x_{1}} \cdots \tilde{\delta}_{x_{n}}$ we therefore obtain

$$
\delta_{x} \gamma \tilde{\sigma} \iota=\delta_{x} \gamma \tilde{\delta}_{x_{1}} \cdots \tilde{\delta}_{x_{n}} \iota=\delta_{x} \gamma \iota \widehat{\delta}_{x_{1}} \cdots \widehat{\delta}_{x_{n}}=\delta_{x} \pi \widehat{\delta}_{x_{1}} \cdots \widehat{\delta}_{x_{n}}=\pi \widehat{\delta}_{x} \widehat{\delta}_{x_{1}} \cdots \widehat{\delta}_{x_{n}}
$$

Hence, for each $\tilde{\sigma} \in \Phi^{*}, y \in \mathcal{Y} \backslash \mathcal{Z}$, and $x \in \mathcal{X}$, the generator $\left(y^{-1} y^{\gamma \chi}\right)^{\delta_{x} \gamma \tilde{\sigma}} \in N$ satisfies $\left(y^{-1} y^{\gamma \chi}\right)^{\delta_{x} \gamma \tilde{\sigma} \iota}=\left(y^{-1} y^{\gamma \chi}\right)^{\pi \widehat{\delta}_{x}} \widehat{\delta}_{x_{1}} \cdots \widehat{\delta}_{x_{n}}=1$ as $y^{-1} y^{\gamma \chi} \in \operatorname{ker}(\pi)$ holds. Therefore $N \subseteq \operatorname{ker}(\iota)$ holds. 
By Lemma 5.24, the homomorphism $\varphi: F(\mathcal{Z}) / N \rightarrow H, g N \mapsto g^{\iota}$ is well-defined and it satisfies that $\psi \varphi=\iota$. We have obtained the following diagram:

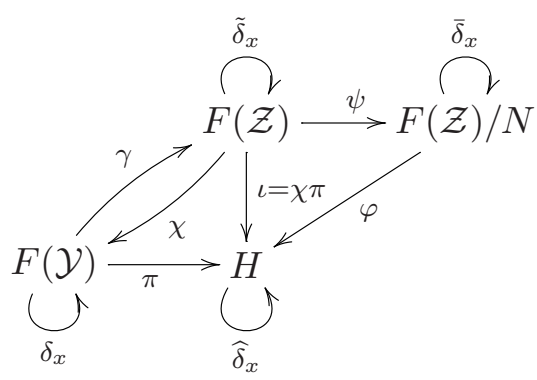

By construction, $F(\mathcal{Z}) / N$ is invariantly $L$-presented by

$$
F(\mathcal{Z}) / N \cong\left\langle\mathcal{Z}|\emptyset|\left\{\tilde{\delta}_{x}\right\}_{\delta_{x} \in \Phi} \mid\left\{\left(y^{-1} y^{\gamma \chi}\right)^{\delta_{x} \gamma}\right\}_{y \in \mathcal{Y} \backslash \mathcal{Z}, \delta_{x} \in \Phi}\right\rangle .
$$

If $[G: H]=\infty$ holds, $|\mathcal{Y} \backslash \mathcal{Z}|$ is infinite. Therefore, the latter $L$-presentation is finite if and only if $[G: H]$ is finite. Our strategy in the proof of Theorem $[$ uses the following

Lemma 5.25 If there exists a finite set $\mathcal{U} \subseteq F(\mathcal{Z})$ with $F(\mathcal{Z}) / N \cong\langle\mathcal{Z}|\emptyset| \tilde{\Phi} \mid \mathcal{U}\rangle$, then $H$ is invariantly finitely L-presented.

Proof. The kernel of $\varphi: F(\mathcal{Z}) / N \rightarrow H$ is generated by the images $r^{\tau \sigma \gamma \psi}=r^{\tau \gamma \psi \bar{\sigma}}$ with $\sigma \in \Phi^{*}$ and $r \in \mathcal{R}$. If $\langle\mathcal{Z}|\emptyset| \tilde{\Phi} \mid \mathcal{U}\rangle$ is an invariant finite $L$-presentation for $F(\mathcal{Z}) / N$, then $H$ is invariantly finitely $L$-presented by $\left\langle\mathcal{Z}|\emptyset| \tilde{\Phi} \mid \mathcal{U} \cup \mathcal{R}^{\tau \gamma}\right\rangle$.

\section{B.5.2 Proofs of Theorem $\mathrm{C}$ and Theorem $\mathrm{D}$}

In this section, we prove Theorem $\mathrm{C}$ and Theorem $\mathrm{D}$;

Proof of Theorem $\mathrm{C}$. Our strategy in the proof of Theorem $\mathrm{C}$ is to construct a normal subgroup $N \unlhd F(\mathcal{Z})$ and to prove that $F(\mathcal{Z}) / N$ is invariantly finitely $L$-presented. Then Lemma 5.25 applies and it shows that $H \leq G$ is invariantly finitely $L$-presented.

Since $G$ is finitely presented, $G / H$ is finitely generated. Moreover, as $G$ splits over $H$, there exists $s_{1}, \ldots, s_{n} \in G$ so that $G / H=\left\langle s_{1} H, \ldots, s_{n} H\right\rangle$ and $S=\left\langle s_{1}, \ldots, s_{n}\right\rangle$ satisfies that $S \cap H=\{1\}$; i.e., $G \cong H \rtimes S$ holds. Because $H$ is finitely generated, there exist $a_{1}, \ldots, a_{m} \in H$ so that $H=\left\langle a_{1}, \ldots, a_{m}\right\rangle$ holds. Then $G=\left\langle a_{1}, \ldots, a_{m}, s_{1}, \ldots, s_{n}\right\rangle$ holds and there exists a finite set of relations $\mathcal{R}$ with $G \cong\left\langle\left\{a_{1}, \ldots, a_{m}, s_{1}, \ldots, s_{n}\right\} \mid \mathcal{R}\right\rangle$. Write $\mathcal{S}=\left\{s_{1}^{ \pm 1}, \ldots, s_{n}^{ \pm 1}\right\}$ and $\mathcal{X}=\left\{a_{1}, \ldots, a_{m}, s_{1}, \ldots, s_{n}\right\}$. Clearly, we can choose a Schreier transversal $\mathcal{T} \subseteq \mathcal{S}^{*}$ whose elements are words over the alphabet $\mathcal{S}$. This yields the Schreier generators

$$
\begin{aligned}
a_{\ell, t}=\gamma\left(t, a_{\ell}\right) & =t a_{\ell}\left(\overline{t a_{\ell}}\right)^{-1}=t a_{\ell} t^{-1} \\
s_{\ell, t} & =\gamma\left(t, s_{\ell}\right)=t s_{\ell}\left(\overline{t s_{\ell}}\right)^{-1}
\end{aligned}
$$

with $t \in \mathcal{T}$. Then $\left\{s_{\ell, t} \mid 1 \leq \ell \leq n, t \in \mathcal{T}\right\} \subseteq \mathcal{S}^{*}$. By Lemma 5.19, the subgroup $H$ is invariantly $L$-presented by $\left\langle\mathcal{Y}|\emptyset|\left\{\delta_{s} \mid s \in \mathcal{S}\right\} \mid \mathcal{R}^{\tau}\right\rangle$ where

$$
\mathcal{Y}=\left\{a_{\ell, t} \mid t \in \mathcal{T}, 1 \leq \ell \leq m\right\} \cup\left\{s_{\ell, t} \neq 1 \mid t \in \mathcal{T}, 1 \leq \ell \leq n\right\}
$$


and $\delta_{s}$ denotes the endomorphism of $F(\mathcal{Y})$ that is induced by conjugation with $s \in \mathcal{S}$. Write $S=\left\langle s_{1}, \ldots, s_{n}\right\rangle \leq F(\mathcal{X})$ and $E=\left\langle a_{1}, \ldots, a_{m}\right\rangle \leq F(\mathcal{X})$. Let $K \unlhd F(\mathcal{X})$ be the kernel of $G$ 's free presentation $F(\mathcal{X}) \rightarrow G$. Then $E K=\langle\mathcal{Y}\rangle$ and $S \cap E K=\left\langle s_{\ell, t} \neq 1\right|$ $1 \leq \ell \leq n, t \in \mathcal{T}\rangle$ are freely generated. For each $s \in \mathcal{S}$, the subgroup $S \cap E K$ is $\delta_{s^{-}}$ invariant since $S \cap E K \unlhd S$ holds. Because $G$ splits over $H$, we have $S \cap H=\{1\}$. Thus the generators $s_{\ell, t} \in S \cap E K$ are contained in the kernel of the free presentation $\pi: F(\mathcal{Y}) \rightarrow H$ which is given by $H$ 's invariant $L$-presentation above. Define $\mathcal{Z}=\left\{a_{1}, \ldots, a_{m}\right\}$ and an embedding

$$
\chi: F(\mathcal{Z}) \rightarrow F(\mathcal{Y}), a_{\ell} \mapsto a_{\ell, 1}
$$

where $1 \in \mathcal{T}$ denotes the trivial element in the Schreier transversal $\mathcal{T}$. For $s \in \mathcal{S}$ and $a_{\ell} \in \mathcal{Z}$, we choose a representative $a_{\ell}^{\chi \delta_{s} \gamma} \in F(\mathcal{Z})$ with

$$
a_{\ell}^{-\chi \delta_{s}}\left(a_{\ell}^{\chi \delta_{s} \gamma}\right)^{\chi} \in \operatorname{ker}(\pi) .
$$

For $s \in \mathcal{S}$, let $\tilde{\delta}_{s} \in F(\mathcal{Z})$ be induced by the map $a_{\ell} \mapsto a_{\ell}^{\chi \delta_{s} \gamma}$ and define $\iota: F(\mathcal{Z}) \rightarrow H$ by $\iota=\chi \pi$. Then Eq. (B.14) yields that $\tilde{\delta}_{s} \iota=\iota \widehat{\delta}_{s}$. In the following, we write $\tilde{\delta}_{t}=\tilde{\delta}_{x_{1}} \cdots \tilde{\delta}_{x_{n}}$ if $t=x_{1} \cdots x_{n} \in \mathcal{S}^{*}$ and each $x_{i} \in \mathcal{S}$. Moreover, we write $X$ for $x^{-1}$ and $T$ for $t^{-1}$. This yields that $a_{\ell, 1}^{\delta_{T}}=t a_{\ell} T=a_{\ell, t}$. Let $\gamma: F(\mathcal{Y}) \rightarrow F(\mathcal{Z})$ be induced by the map

$$
\gamma:\left\{\begin{array}{cll}
a_{\ell, t} & \mapsto a_{\ell}^{\tilde{\delta}_{T}}, & \text { for each } 1 \leq \ell \leq m \text { and } t \in \mathcal{T} \\
s_{\ell, t} & \mapsto 1, & \text { for each } 1 \leq \ell \leq n \text { and } t \in \mathcal{T}
\end{array}\right.
$$

For each $1 \leq \ell \leq m, 1 \leq k \leq n$, and $t \in \mathcal{T}$, this yields

$$
\left(a_{\ell, t}\right)^{\gamma \iota}=a_{\ell}^{\tilde{\delta}_{T}^{\iota}}=a_{\ell}^{\iota \widehat{\delta}_{T}}=a_{\ell}^{\chi \pi \widehat{\delta}_{T}}=a_{\ell, 1}^{\delta_{T} \pi}=a_{\ell, t}^{\pi} \quad \text { and } \quad\left(s_{k, t}\right)^{\gamma \iota}=1^{\iota}=1=\left(s_{k, t}\right)^{\pi} .
$$

Thus $\gamma \iota=\pi$. Define the normal subgroup

$$
N=\left\langle\bigcup_{\tilde{\sigma} \in \tilde{\Phi}^{*}}\left(\left\{\left(y^{-1} y^{\gamma \chi}\right)^{\delta_{s} \gamma}\right\}_{y \in \mathcal{Y} \backslash \mathcal{Z}, s \in \mathcal{S}}\right)^{\tilde{\sigma}}\right\rangle^{F(\mathcal{Y})}
$$

where $\tilde{\Phi}=\left\{\tilde{\delta}_{s} \mid s \in \mathcal{S}\right\}$. For $t \in \mathcal{T}$ and $s \in \mathcal{S}$, we have that

$$
\left(s_{\ell, t}^{-1} s_{\ell, t}^{\gamma \chi}\right)^{\delta_{s} \gamma}=s_{\ell, t}^{-\delta_{s} \gamma}\left(s_{\ell, t}^{\gamma}\right)^{\tilde{\delta}_{s}}=1
$$

as the subgroup $S \cap E K=\left\langle s_{\ell, t} \mid t \in \mathcal{T}, 1 \leq \ell \leq n\right\rangle$ is $\delta_{s}$-invariant and it is contained in the kernel of $\gamma$. This yields that

$$
N=\left\langle\bigcup_{\tilde{\sigma} \in \tilde{\Phi}^{*}}\left(\left\{\left(a_{\ell, t}^{-1} a_{\ell, t}^{\gamma \chi}\right)^{\delta_{s} \gamma}\right\}_{1 \leq \ell \leq m, t \in \mathcal{T} \backslash\{1\}, s \in \mathcal{S}}\right)^{\tilde{\sigma}}\right\rangle^{F(\mathcal{Z})}
$$

For $t \in \mathcal{T}$ and $x \in \mathcal{S}$ with $x t \in \mathcal{T}$, we also have that

$$
\left(a_{\ell, t}^{-1} a_{\ell, t}^{\gamma \chi}\right)^{\delta_{X} \gamma}=a_{\ell, t}^{-\delta_{X} \gamma} a_{\ell, t}^{\gamma \tilde{\delta}_{X}}=a_{\ell, x t}^{-\gamma} a_{\ell, t}^{\gamma \tilde{\delta}_{X}}=a_{\ell}^{-\tilde{\delta}_{T X}} a_{\ell}^{\tilde{\delta}_{T} \tilde{\delta}_{X}}=1 .
$$


It therefore suffices to consider the generators $\left(a_{\ell, t}^{-1} a_{\ell, t}^{\gamma \chi}\right)^{\delta_{X} \gamma} \in N$ with $1 \leq \ell \leq m, t \in \mathcal{T}$, and $x \in \mathcal{S}$ but $x t \notin \mathcal{T}$. Since $G / H \cong S / S \cap E K$ is a finitely presented group, there exists a finite monoid presentation

$$
S / S \cap E K \cong\left\langle\mathcal{S} \mid\left(U_{1}, V_{1}\right), \ldots,\left(U_{p}, V_{p}\right)\right\rangle .
$$

The monoid congruence $\sim$ induced by this presentation is the reflexive, symmetric, and transitive closure of the binary relation $\sim$ that is defined by $x \sim y$ if there exist $A, B \in \mathcal{S}^{*}$ and $1 \leq i \leq p$ so that $x=A U_{i} B$ and $y=A V_{i} B$ hold. Define

$$
M=\left\langle\bigcup_{\tilde{\sigma} \in \tilde{\Phi}^{*}}\left(\left\{\left(a_{\ell}^{-\tilde{\delta}_{U_{i}}} a_{\ell}^{\tilde{\delta}_{V_{i}}}\right)\right\}_{1 \leq \ell \leq m, 1 \leq i \leq p}\right)^{\tilde{\sigma}}\right\rangle^{F(\mathcal{Z})} .
$$

Suppose that $u \sim v$ holds. Then there exist $A_{i}, B_{i}, L_{i} \in \mathcal{S}^{*}$ so that $u=L_{1} \sim \ldots \sim L_{q}=v$ with $L_{i}=A_{i} U_{\ell_{i}} B_{i}$ and $L_{i+1}=A_{i} V_{\ell_{i}} B_{i}$ (or $L_{i}=A_{i} V_{\ell_{i}} B_{i}$ and $L_{i+1}=A_{i} U_{\ell_{i}} B_{i}$ ). Note that

$$
a_{\ell}^{\tilde{\delta}_{A_{i}} \tilde{\delta}_{\ell_{\ell_{i}}} \tilde{\delta}_{B_{i}}}=\left(a_{\ell}^{\tilde{\delta}_{A_{i}}}\right)^{\tilde{\delta}_{U_{i}} \tilde{\delta}_{B_{i}}}=w_{\ell}\left(a_{1}, \ldots, a_{m}\right)^{\tilde{\delta}_{U_{\ell}} \tilde{\delta}_{B_{i}}}=w_{\ell}\left(a_{1}^{\tilde{\delta}_{\ell_{i}}}, \ldots, a_{m} \tilde{\delta}_{\ell_{\ell_{i}}} \tilde{\delta}_{B_{i}}\right.
$$

for some word $w_{\ell}\left(a_{1}, \ldots, a_{m}\right)=a_{\ell}^{\tilde{\delta}_{A_{i}}} \in F(\mathcal{Z})$. The normal subgroup $M$ yields that

$$
\left(a_{\ell}^{\tilde{\delta}_{A_{i}}}\right)^{\tilde{\delta}_{U_{\ell_{i}}}}=w_{\ell}\left(a_{1}^{\tilde{\delta}_{U_{\ell_{i}}}}, \ldots, a_{m} \tilde{\delta}_{\ell_{\ell_{i}}}\right)=w_{\ell}\left(a_{1}^{\tilde{\delta}_{V_{\ell_{i}}}}, \ldots, a_{m}^{\tilde{\delta}_{V_{\ell_{i}}}}\right) \cdot h=a_{\ell}^{\tilde{\delta}_{A_{i}} \tilde{\delta}_{V_{\ell_{i}}}} \cdot h
$$

for some $h \in M$. By construction, $M$ is $\tilde{\Phi}^{*}$-invariant and thus

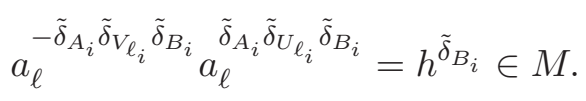

This shows that, if $u \sim v$ holds, we have $a_{\ell}^{-\tilde{\delta}_{u}} a_{\ell}^{\tilde{\delta}_{v}} \in M$. Suppose that, for $t \in \mathcal{T}$ and $x \in \mathcal{S}, x t \notin \mathcal{T}$ holds. Then there exists $u=\overline{x t} \in \mathcal{T}$ with $u \sim x t$. Write $U$ for $u^{-1}$. Since $S \cap E K \unlhd S$ holds, there exists $h \in S \cap E K \subseteq \operatorname{ker}(\gamma)$ so that $x t=h u$. This yields that $a_{\ell, t}^{\delta_{X}}=x t a_{\ell} T X=h u a_{\ell} U h^{-1}=h a_{\ell, u} h^{-1}$ and $a_{\ell, t}^{\delta_{X} \gamma}=a_{\ell, u}^{\gamma}=a_{\ell}^{\tilde{\delta}_{U}}$. Since $u \sim x t$ and $U \sim T X$ hold, we obtain

$$
\left(a_{\ell, t}^{-1} a_{\ell, t}^{\gamma \chi}\right)^{\delta_{X} \gamma}=a_{\ell, u}^{-\gamma} a_{\ell}^{\tilde{\delta}_{T} \tilde{\delta}_{X}}=a_{\ell}^{-\tilde{\delta}_{U}} a_{\ell}^{\tilde{\delta}_{T} \tilde{\delta}_{X}} \in M .
$$

Thus $N \subseteq M$. It suffices to show that $M \subseteq N$ holds. Since $M$ and $N$ are both normal subgroups of $F(\mathcal{Z})$ and both are $\tilde{\Phi}^{*}$-invariant, it suffices to prove that $a_{\ell}^{-\tilde{\delta}_{U_{i}}} a_{\ell} \tilde{\delta}_{V_{i}} \in N=$ $\operatorname{ker}(\psi)$ holds. Since $\tilde{\delta}_{s} \psi=\psi \bar{\delta}_{s}$ and $\chi \gamma=\operatorname{id}_{F(\mathcal{Z})}$ hold, we have that

$$
\begin{aligned}
\left(a_{\ell}^{-\tilde{\delta}_{U_{i}}} a_{\ell}^{\tilde{\delta}_{V_{i}}}\right)^{\psi} & =a_{\ell}^{-\psi \bar{\delta}_{U_{i}}} a_{\ell}^{\psi \bar{\delta}_{V_{i}}}=a_{\ell}^{-\chi \gamma \psi \bar{\delta}_{U_{i}}} a_{\ell}^{\chi \gamma \psi \bar{\delta}_{V_{i}}}=a_{\ell, 1}^{-\gamma \psi \bar{\delta}_{U_{i}}} a_{\ell, 1}^{\gamma \psi \bar{\delta}_{V_{i}}} \\
& =a_{\ell, 1}^{-\delta_{U_{i}} \gamma \psi} a_{\ell, 1}^{\delta_{V_{i}} \gamma \psi}=\left(a_{\ell, 1}^{-\delta_{U_{i}}} a_{\ell, 1}^{\delta_{V_{i}}}\right)^{\gamma \psi} .
\end{aligned}
$$

As $S \cap E K \unlhd S$ and $\mathcal{T} \subseteq S$ hold, there exist $h \in S \cap E K=\left\langle s_{\ell, t} \mid 1 \leq \ell \leq n, t \in \mathcal{T}\right\rangle$ and $t=\overline{U_{i}^{-1}} \in \mathcal{T}$ with $U_{i}^{-1}=h t$. Thus $a_{\ell, 1}^{\delta_{U_{i}}}=U_{i}^{-1} a_{\ell} U_{i}=h t a_{\ell} t^{-1} h^{-1}=h a_{\ell, t} h^{-1}$. 
Since $h \in \operatorname{ker}(\gamma)$ holds, we obtain $\left(a_{\ell, 1}^{\delta_{U_{i}}}\right)^{\gamma}=a_{\ell, t}^{\gamma}$. Since $U_{i} \sim V_{i}$ holds, we also have that $\overline{V_{i}^{-1}}=t$. Similarly, we obtain $\left(a_{\ell, 1}^{\delta_{V_{i}}}\right)^{\gamma}=a_{\ell, t}^{\gamma}$. Thus $a_{\ell, 1}^{-\delta_{U_{i}}} a_{\ell, 1}^{\delta_{V_{i}}} \in \operatorname{ker}(\gamma)$ and so $\left(a_{\ell}^{-\tilde{\delta}_{U_{i}}} a_{\ell}^{\tilde{\delta}_{V_{i}}}\right)^{\psi}=1$ or $a_{\ell}^{-\tilde{\delta}_{U_{i}}} a_{\ell}^{\tilde{\delta}_{V_{i}}} \in N$. Thus $M=N$. This shows that that factor group $F(\mathcal{Z}) / N$ is invariantly finitely $L$-presented and so is our subgroup $H$.

Even if $G / H$ is free, the finite $L$-presentation of $F(\mathcal{Z}) / N$ in the proof of Theorem $\mathrm{C}$ contains the relations of a monoid presentation of the free group. It is not clear whether or not these relations can be omitted as was done in [22]. However, the result in [22] is a consequence of Theorem $\mathrm{C}$ even if these relations are not redundant:

Theorem 5.26 (Benli [22]) Every finitely generated normal subgroup of a finitely presented group is invariantly finitely L-presented if the quotient is infinite cyclic.

Proof. Since the quotient is free, the finitely presented group splits over its finitely generated normal subgroup and thus, by Theorem C, the subgroup is invariantly finitely $L$-presented.

Even if the finitely presented group does not split over its finitely generated subgroup, the subgroup is possibly invariantly finitely $L$-presented:

Theorem 5.27 Every finitely generated normal subgroup of a finitely presented group is invariantly finitely L-presented if the quotient is free abelian with rank two.

Proof. Let $G$ be a finitely presented group and let $H \unlhd G$ be finitely generated so that $G / H \cong \mathbb{Z} \times \mathbb{Z}$ holds. By Lemma 5.25, it suffices to construct a factor group $F(\mathcal{Z}) / N$ which is invariantly finitely $L$-presented. Since $G / H \cong \mathbb{Z} \times \mathbb{Z}$ holds, there exists $t, u \in G$ so that $G / H=\langle t H, u H\rangle$ holds. Moreover, as $H$ is finitely generated, there exist $a_{1}, \ldots, a_{m} \in H$ so that $H=\left\langle a_{1}, \ldots, a_{m}\right\rangle$ holds. Then $G=\left\langle a_{1}, \ldots, a_{m}, t, u\right\rangle$ holds and there exists a finite set of relations $\mathcal{R}$ with $G \cong\left\langle\left\{a_{1}, \ldots, a_{m}, t, u\right\} \mid \mathcal{R}\right\rangle$. We choose as Schreier transversal $\mathcal{T}=\left\{t^{i} u^{j} \mid i, j \in \mathbb{Z}\right\}$. Then, by Lemma 5.19, the subgroup $H$ is invariantly $L$-presented by $\left\langle\mathcal{Y}|\emptyset|\left\{\delta_{u}, \delta_{U}, \delta_{t}, \delta_{T}\right\} \mid \mathcal{R}^{\tau}\right\rangle$ where $\delta_{x}$ denotes the endomorphism of the free group $F(\mathcal{Y})$ that is induced by conjugation with $x \in\left\{u, U=u^{-1}, t, T=t^{-1}\right\}, \tau$ denotes the Reidemeister rewriting, and $\mathcal{Y}=\left\{a_{\ell, i, j}, t_{l, k} \mid i, j, k, l \in \mathbb{Z}, k \neq 0\right\}$ are the following Schreier generators:

$$
\begin{aligned}
a_{\ell, i, j} & =\gamma\left(t^{i} u^{j}, a_{\ell}\right)=t^{i} u^{j} a_{\ell} u^{-j} t^{-i} \\
t_{i, j} & =\gamma\left(t^{i} u^{j}, t\right)=t^{i} u^{j} t u^{-j} t^{-1} t^{-i} \\
u_{i, j} & =\gamma\left(t^{i} u^{j}, u\right)=t^{i} u^{j} u u^{-j} u^{-1} t^{-i}
\end{aligned}
$$

Note that $t_{i, j}=1$ if and only if $j=0$ while $u_{i, j}=1$ for each $i, j \in \mathbb{Z}$. The endomorphisms $\delta_{t}$ and $\delta_{T}$ are induced by the maps

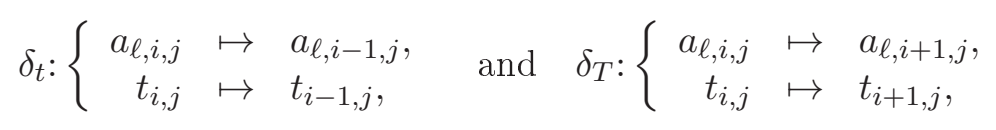


for each $i, j \in \mathbb{Z}$; while $\delta_{u}$ and $\delta_{U}$ are induced by the maps

$$
\delta_{u}:\left\{\begin{array}{rlrl}
a_{\ell, i, j} & \mapsto\left(a_{\ell, i, j-1}\right)^{t_{i-1,-1}^{-1} \cdots t_{0,-1}^{-1},} & & i \geq 0, j \in \mathbb{Z}, \\
a_{\ell,-i, j} & \mapsto\left(a_{\ell,-i, j-1}\right)^{t_{-i,-1} \cdots t_{-1,-1},} & i \geq 0, j \in \mathbb{Z}, \\
t_{i, j} & \mapsto\left(t_{i, j-1} t_{i,-1}^{-1}\right)^{t_{i-1,-1} \cdots t_{0,-1}^{-1},} & i \geq 0, j \in \mathbb{Z}, \\
t_{-i, j} & \mapsto\left(t_{-i, j-1} t_{-i,-1}^{-1}\right)^{t_{-i,-1} \cdots t_{-1,-1}}, & i \geq 0, j \in \mathbb{Z},
\end{array}\right.
$$

and

$$
\delta_{U}:\left\{\begin{array}{rlrl}
a_{\ell, i, j} & \mapsto\left(a_{\ell, i, j+1}\right)^{t_{i-1,1}^{-1} \cdots t_{0,1}^{-1}}, & & i \geq 0, j \in \mathbb{Z}, \\
a_{\ell,-i, j} & \mapsto\left(a_{\ell,-i, j+1}\right)^{t_{-i, 1} \cdots t_{-1,1}}, & & i \geq 0, j \in \mathbb{Z}, \\
t_{i, j} & \mapsto\left(t_{i, j+1} t_{i, 1}^{-1}\right)^{t_{i-1,1}^{-1} \cdots t_{0,1}^{-1}}, & & i \geq 0, j \in \mathbb{Z}, \\
t_{-i, j} & \mapsto\left(t_{-i, j+1} t_{-i, 1}^{-1}\right)^{t_{-i, 1} \cdots t_{-1,1}}, & i \geq 0, j \in \mathbb{Z} .
\end{array}\right.
$$

We will construct an invariant finite $L$-presentation for the subgroup $H$ with generators $\mathcal{Z}=\left\{a_{1}, \ldots, a_{m}\right\} \cup\left\{t_{1}\right\}$. Define an embedding $\chi: F(\mathcal{Z}) \rightarrow F(\mathcal{Y})$ that is induced by the map

$$
\chi:\left\{\begin{aligned}
a_{\ell} & \mapsto a_{\ell, 0,0}, \quad \text { for each } 1 \leq \ell \leq m \\
t_{1} & \mapsto t_{0,1} .
\end{aligned}\right.
$$

Write $\Phi=\left\{\delta_{t}, \delta_{T}, \delta_{u}, \delta_{U}\right\}$. For $y \in \mathcal{Z}$ and $\delta \in \Phi$, choose $y^{\chi \delta \gamma} \in F(\mathcal{Z})$ with

$$
y^{-\chi \delta}\left(y^{\chi \delta \gamma}\right)^{\chi} \in \operatorname{ker}(\pi) .
$$

Define $\iota: F(\mathcal{Z}) \rightarrow H$ by $\iota=\chi \pi$ where $\pi$ denotes the free presentation $\pi: F(\mathcal{Y}) \rightarrow H$ that is given by $H$ 's invariant $L$-presentation above. For each $\delta \in \Phi$, define an endomorphism $\tilde{\delta}: F(\mathcal{Z}) \rightarrow F(\mathcal{Z})$ that is induced by the map $y \mapsto y^{\chi \delta \gamma}$. Then, for each $\delta \in \Phi$ and $y \in \mathcal{Z}$, we obtain

$$
y^{\widehat{\delta}}=y^{\chi \pi \widehat{\delta}}=y^{\chi \delta \pi}=\left(y^{\chi \delta}\right)^{\pi}=\left(y^{\chi \delta \gamma \chi}\right)^{\pi}=\left(y^{\chi \delta \gamma}\right)^{\chi \pi}=y^{\tilde{\delta} \iota}
$$

and thus $\tilde{\delta} \iota=\gamma \widehat{\delta}$. Write $\mathcal{X}=\left\{a_{1}, \ldots, a_{m}, t, u\right\}$ and consider the following subgroups of the free group $F(\mathcal{X})$ : Let $E=\left\langle a_{1}, \ldots, a_{m}\right\rangle$ and $S=\langle t, u\rangle$ be finitely generated subgroups of $F(\mathcal{X})$. Furthermore, let $K \unlhd F(\mathcal{X})$ be the kernel of $G$ 's free presentation. Then $G \cong F(\mathcal{X}) / K$ and $H \cong E K / K$. Moreover, the normal subgroup $E K \unlhd F(\mathcal{X})$ is supplemented by the finitely generated free group $S$; i.e., $F(\mathcal{X})=S E K$ holds. Thus $G / H \cong F(\mathcal{X}) / E K \cong S / S \cap E K$. Since $G / H$ is finitely presented, the free subgroup $S \cap E K$ is finitely generated as a normal subgroup. The Schreier generators $\mathcal{Y}$ yield that the subgroups

$$
E K=\langle\mathcal{Y}\rangle \quad \text { and } \quad S \cap E K=\left\langle t_{i, j} \mid i, j \in \mathbb{Z}, j \neq 0\right\rangle
$$

are freely generated. Moreover, we have that

$$
\begin{aligned}
S \cap E K & =\left\langle t_{i, j+1} t_{i, j}^{-1} \mid i, j \in \mathbb{Z}\right\rangle \\
& =\left\langle\ldots t_{i,-2} t_{i,-3}^{-1}, t_{i,-1} t_{i,-2}^{-1}, t_{i,-1}^{-1}, t_{i, 1}, t_{i, 2} t_{i, 1}^{-1}, t_{i, 3} t_{i, 2}^{-1}, \ldots \mid i \in \mathbb{Z}\right\rangle .
\end{aligned}
$$


The latter subgroup is freely generated as the homomorphism $\psi$ that is induced by the map

$$
\psi: S \cap E K \rightarrow S \cap E K,\left\{\begin{aligned}
t_{i, j} & \mapsto t_{i, j+1} t_{i, j}^{-1}, \quad j<-1 \\
t_{i,-1} & \mapsto t_{i,-1}^{-1} \\
t_{i, 1} & \mapsto t_{i, 1} \\
t_{i, j} & \mapsto t_{i, j} t_{i, j-1}^{-1}, \quad j>1
\end{aligned}\right.
$$

is an automorphism of $S \cap E K$ whose inverse is induced by the map

$$
\psi^{-1}: S \cap E K \rightarrow S \cap E K,\left\{\begin{aligned}
t_{i, j} & \mapsto t_{i, j}^{-1} t_{i, j+1}^{-1} \cdots t_{i,-1}^{-1}, \quad j<-1 \\
t_{i,-1} & \mapsto t_{i,-1}^{-1}, \\
t_{i, 1} & \mapsto t_{i, 1} \\
t_{i, j} & \mapsto t_{i, j} t_{i, j-1} \cdots t_{i, 1}, \quad j>1 .
\end{aligned}\right.
$$

Note that we have

$$
t_{i, j+1} t_{i, j}^{-1}=t^{i} u^{j+1} t u^{-j-1} t^{-1} t^{-i} \cdot\left(t^{i} u^{j} t u^{-j} t^{-1} t^{-i}\right)^{-1}=\left(t_{0,1}\right)^{u^{j} t^{i}} .
$$

In fact, every element in $S \cap E K$ has a unique representation as a word in the basis $\left\{t^{i} u^{j} \cdot t_{0,1} \cdot u^{-j} t^{-i} \mid i, j \in \mathbb{Z}\right\}$ where $t_{0,1}=u t U T$ is a normal generator of $S \cap E K=\left\langle t_{0,1}\right\rangle^{S}$. More precisely, for $i \geq 0$ and $j>0$, we have the following representatives in free subgroup $S \cap E K \leq F(\mathcal{Y})$ :

$$
\begin{aligned}
t_{i, j} & =\left(t_{0,1}^{\delta_{U}^{j-1}} \cdot t_{0,1}^{\delta_{U}^{j-2}} \cdots t_{0,1}\right)^{\delta_{T}^{i}} \\
t_{-i, j} & =\left(t_{0,1}^{\delta_{U}^{j-1}} \cdot t_{0,1}^{\delta_{U}^{j-2}} \cdots t_{0,1}\right)^{\delta_{t}^{i}} \text { and }
\end{aligned} \begin{aligned}
t_{i,-j} & =\left(t_{0,1}^{-\delta_{u}^{j}} \cdot t_{0,1}^{-\delta_{u}^{j-1}} \cdots t_{0,1}^{-\delta_{u}}\right)^{\delta_{T}^{i}} \\
t_{-i,-j} & =\left(t_{0,1}^{-\delta_{u}^{j}} \cdot t_{0,1}^{-\delta_{u}^{j-1}} \cdots t_{0,1}^{-\delta_{u}}\right)^{\delta_{t}^{i}}
\end{aligned}
$$

The Schreier generators $a_{\ell, i, j}$ are conjugates of the generators $a_{\ell, 0,0}$ so that

$$
\begin{aligned}
& a_{\ell, i, j}=\left(a_{\ell, 0,0}\right)^{\delta_{U}^{j} \delta_{T}^{i}} \\
& a_{\ell,-i, j}=\left(a_{\ell, 0,0}\right)^{\delta_{U}^{j} \delta_{t}^{i}} \\
& a_{\ell, i,-j}=\left(a_{\ell, 0,0}\right)^{\delta_{u}^{j} \delta_{T}^{i}} \\
& a_{\ell,-i,-j}=\left(a_{\ell, 0,0}\right)^{\delta_{u}^{j} \delta_{t}^{i}} .
\end{aligned}
$$

In particular, we can choose the following basis $\widehat{\mathcal{Y}}$ for the free subgroup $E K$ :

$$
\widehat{\mathcal{Y}}=\left\{\left(a_{\ell, 0,0}\right)^{\delta_{U}^{j} \delta_{T}^{i}}, \ldots\left(a_{\ell, 0,0}\right)^{\delta_{u}^{j} \delta_{t}^{i}},\left(t_{0,1}\right)^{\delta_{U}^{j} \delta_{T}^{i}}, \ldots,\left(t_{0,1}\right)^{\delta_{u}^{j} \delta_{t}^{i}}\right\}_{i, j \geq 0} .
$$

Define $\gamma: F(\widehat{\mathcal{Y}}) \rightarrow F(\mathcal{Z})$ to be induced by the map

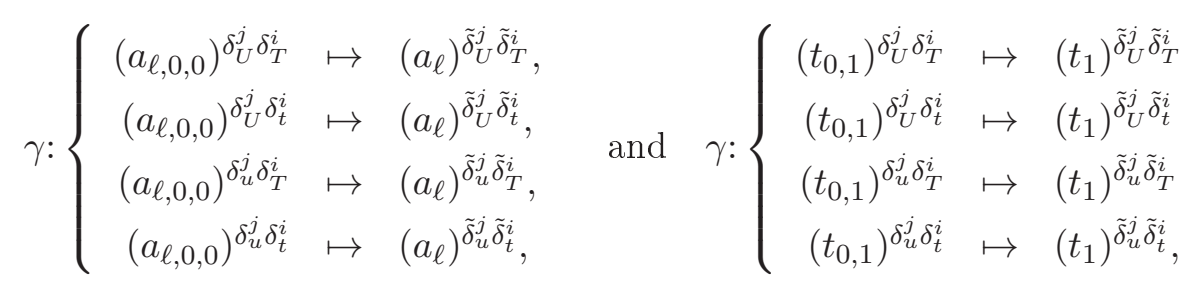


where $i, j \geq 0$. Then $\gamma$ acts on the Schreier generators $\mathcal{Y}$ as follows:

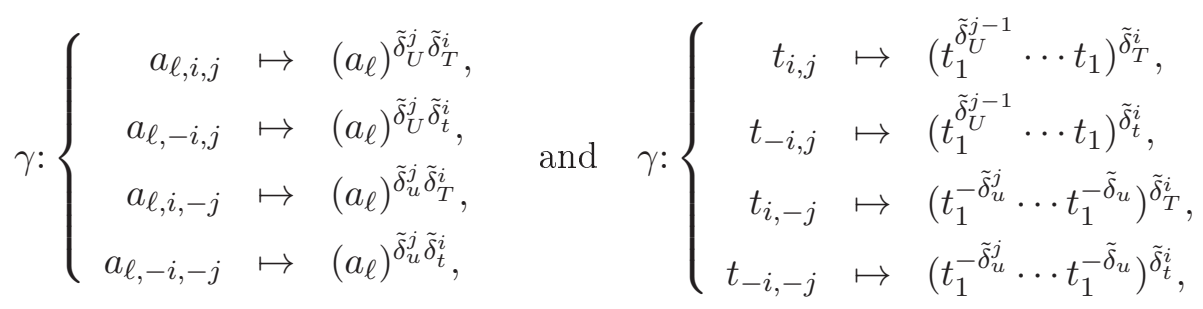

where $i \geq 0$ and $j>0$. For $i \geq 0$ and $j>0$, the element $t_{i, j} \in \mathcal{Y}$ is mapped by $\gamma \iota$ to

$$
\begin{aligned}
t_{i, j}^{\gamma \iota} & =\left(t_{1}^{\tilde{\delta}_{U}^{j-1}} \cdots t_{1}\right)^{\tilde{\delta}_{T}^{i} \iota}=\left(t_{1}^{\tilde{\delta}_{U}^{j-1}} \tilde{\delta}_{T}^{i} \cdots t_{1}^{\tilde{\delta}_{T}^{i}}\right)^{\iota}=t_{1}^{\iota_{U}^{j-1} \widehat{\delta}_{T}^{i}} \cdots t_{1}^{\iota \widehat{\delta}_{T}^{i}} \\
& =\left(t_{1}^{\chi \pi \widehat{\delta}_{U}^{j-1} \widehat{\delta}_{T}^{i}} \cdots t_{1}^{\chi \pi \widehat{\delta}_{T}^{i}}\right)=\left(t_{0,1}^{\delta_{U}^{j-1}} \delta_{T}^{i} \cdots t_{T, 1}^{\delta_{T}^{i}}\right)^{\pi}=\left(t_{i, j}\right)^{\pi}
\end{aligned}
$$

because $\tilde{\delta} \iota=\iota \widehat{\delta}$ holds. Similarly, we obtain that $a_{\ell, i, j}^{\gamma \iota}=a_{\ell, i, j}^{\pi}$ holds. Thus $\gamma \iota=\pi$. Define the normal subgroup

$$
N=\left\langle\bigcup_{\sigma \in \tilde{\Phi}^{*}}\left(\left\{\left(y^{-1} y^{\gamma \chi}\right)^{\delta \gamma}\right\}_{y \in \mathcal{Y} \backslash \mathcal{Z}, \delta \in \Phi}\right)^{\sigma}\right\rangle^{F(\mathcal{Z})} .
$$

We prove that $F(\mathcal{Z}) / N$ is invariantly finitely $L$-presented so that Lemma 5.25 applies. For $i \geq 0$ and $j>0$, it holds that

$$
\begin{aligned}
& \left(t_{i, j}^{-1} t_{i, j}^{\gamma \chi}\right)^{\delta_{T} \gamma}=t_{i+1, j}^{-\gamma} t_{i, j}^{\gamma \tilde{\delta}_{T}}=\left(t_{1}^{\tilde{\delta}_{U}^{j-1}} \cdots t_{1}\right)^{-\tilde{\delta}_{T}^{i+1}}\left(t_{1}^{\tilde{\delta}_{U}^{j-1}} \cdots t_{1}\right)^{\tilde{\delta}_{T}^{i} \tilde{\delta}_{T}}=1, \\
& \left(t_{-i, j}^{-1} t_{-i, j}^{\gamma \chi}\right)^{\delta_{t} \gamma}=t_{-i-1, j}^{-\gamma} t_{-i, j}^{\gamma \tilde{\delta}_{t}}=\left(t_{1}^{\tilde{\delta}_{U}^{j-1}} \cdots t_{1}\right)^{-\tilde{\delta}_{t}^{i+1}}\left(t_{1}^{\tilde{\delta}_{U}^{j-1}} \cdots t_{1}\right)^{\tilde{\delta}_{t}^{i} \tilde{\delta}_{t}}=1, \\
& \left(t_{i,-j}^{-1} t_{i,-j}^{\gamma \chi}\right)^{\delta_{T} \gamma}=t_{i+1,-j}^{-\gamma} t_{i,-j}^{\gamma \tilde{\delta}_{T}}=\left(t_{1}^{-\tilde{\delta}_{u}^{j}} \cdots t_{1}^{-\tilde{\delta}_{u}}\right)^{-\tilde{\delta}_{T}^{i+1}}\left(t_{1}^{-\tilde{\delta}_{u}^{j}} \cdots t_{1}^{-\tilde{\delta}_{u}}\right)^{\tilde{\delta}_{T}^{i}} \tilde{\delta}_{T}=1 \text {, } \\
& \left(t_{-i,-j}^{-1} t_{-i,-j}^{\gamma \chi}\right)^{\delta_{t} \gamma}=t_{-i-1,-j}^{-\gamma} t_{-i,-j}^{\gamma \tilde{\delta}_{t}}=\left(t_{1}^{-\tilde{\delta}_{u}^{j}} \cdots t_{1}^{-\tilde{\delta}_{u}}\right)^{-\tilde{\delta}_{t}^{i+1}}\left(t_{1}^{-\tilde{\delta}_{u}^{j}} \cdots t_{1}^{-\tilde{\delta}_{u}}\right)^{\tilde{\delta}_{t}^{i}} \tilde{\delta}_{t}=1 .
\end{aligned}
$$

For $i=0$, we also have that

$$
\begin{aligned}
\left(t_{0, j}^{-1} t_{0, j}^{\gamma \chi}\right)^{\delta_{U} \gamma} & =\left(t_{0, j+1} t_{0,1}^{-1}\right)^{-\gamma} t_{0, j}^{\gamma \tilde{\delta}_{U}}=\left(t_{1}^{\tilde{\delta}_{U}^{j}} \cdots t_{1}^{\tilde{\delta}_{U}} \cdot t_{1} \cdot t_{1}^{-1}\right)^{-1}\left(t_{1}^{\tilde{\delta}_{U}^{j-1}} \cdots t_{1}\right)^{\tilde{\delta}_{U}}=1, \\
\left(t_{0,-j}^{-1} t_{0,-j}^{\gamma \chi}\right)^{\delta_{u} \gamma} & =\left(t_{0,-j-1} t_{0,-1}^{-1}\right)^{-\gamma} t_{0,-j}^{\gamma \tilde{\delta}_{u}}=\left(t_{1}^{-\tilde{\delta}_{u}^{j+1}} \cdots t_{1}^{-\tilde{\delta}_{u}} \cdot t_{1}^{\tilde{\delta}_{u}}\right)^{-1}\left(t_{1}^{-\tilde{\delta}_{u}^{j}} \cdots t_{1}^{\tilde{\delta}_{u}}\right)^{\tilde{\delta}_{u}}=1 .
\end{aligned}
$$

However, we also need to consider the image $t_{i, j}^{\delta_{U}}=t_{0,1}^{\delta_{T}^{i} \delta_{U}}$ with $i>0$. Notice that in the finitely presented monoid $S / S \cap E K$ the following holds:

$$
\begin{aligned}
& T U=U T \cdot t u T U=U T \cdot(u t U T)^{-1}=U T \cdot t_{0,1}^{-1}, \\
& T u=u T \cdot t U T u=u T \cdot(u t U T)^{\delta_{u}}=u T \cdot t_{0,1}^{\delta_{u}}, \\
& t U=U \cdot(u t U T) \cdot t=U \cdot t_{0,1} \cdot t=U t \cdot t_{0,1}^{\delta_{t}} \text {, } \\
& t u=u \cdot(U t u T) \cdot t=u \cdot t_{0,-1} \cdot t=u t \cdot t_{0,1}^{-\delta_{u} \delta_{t}} .
\end{aligned}
$$

Denote by $\Delta(x): F(\mathcal{Y}) \rightarrow F(\mathcal{Y}), g \mapsto x^{-1} g x$ the inner automorphism of $F(\mathcal{Y})$ that is induced by conjugation with $x \in F(\mathcal{Y})$. Then $\delta \in \Phi=\left\{\delta_{u}, \delta_{U}, \delta_{t}, \delta_{T}\right\}$ satisfy

$$
\begin{aligned}
\delta_{T} \delta_{U} & =\delta_{U} \delta_{T} \cdot \Delta\left(t_{0,1}^{-1}\right), \\
\delta_{T} \delta_{u} & =\delta_{u} \delta_{T} \cdot \Delta\left(t_{0,1}^{\delta_{u}}\right),
\end{aligned} \quad \text { and } \quad \begin{aligned}
\delta_{t} \delta_{U} & =\delta_{U} \delta_{t} \cdot \Delta\left(t_{0,1}^{\delta_{t}}\right), \\
\delta_{t} \delta_{u} & =\delta_{u} \delta_{t} \cdot \Delta\left(t_{0,1}^{-\delta_{u} \delta_{t}}\right) .
\end{aligned}
$$


We prove that $F(\mathcal{Z}) / N$ is invariantly finitely $L$-presented by $\left\langle\left\{a_{1}, \ldots, a_{m}, t_{1}\right\}|\emptyset| \tilde{\Phi} \mid \mathcal{V}\right\rangle$ where the iterated relations in $\mathcal{V}$ are given by

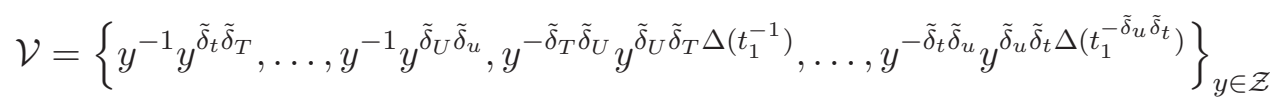

that is, we prove that $M=\left\langle\bigcup_{\tilde{\sigma} \in \tilde{\Phi}} \mathcal{V}^{\tilde{\sigma}}\right\rangle^{F(\mathcal{Z})}$ and $N$ coincide. We first note that

$$
\begin{aligned}
N \ni\left(t_{1,1}^{-1} t_{1,1}^{\gamma \chi}\right)^{\delta_{U} \gamma} & =t_{1,1}^{-\delta_{U} \gamma} t_{1,1}^{\tilde{\gamma}_{\delta_{U}}}=t_{0,1}^{-\delta_{T} \delta_{U} \gamma} t_{1}^{\tilde{\delta}_{T} \tilde{\delta}_{U}}=t_{0,1}^{-\delta_{U} \delta_{T} \cdot \Delta\left(t_{0,1}^{-1}\right) \gamma} t_{1}^{\tilde{\delta}_{T} \tilde{\delta}_{U}} \\
& =t_{0,1}^{-\delta_{U} \delta_{T} \gamma \cdot \Delta\left(t_{1}^{-1}\right)} t_{1}^{\tilde{\delta}_{T} \tilde{\delta}_{U}}=t_{1}^{-\tilde{\delta}_{U} \tilde{\delta}_{T} \cdot \Delta\left(t_{1}^{-1}\right)} t_{1}^{\tilde{\delta}_{T} \tilde{\delta}_{U}} \in \mathcal{V} .
\end{aligned}
$$

Similar computations show that the elements in $\mathcal{V}$ appear a among the normal generators of $N$. Thus $M \subseteq N$. On the other hand, for $i>0$ and $j>0$, we have that

$$
\begin{aligned}
& t_{i, j}^{\delta_{U} \gamma}=\left(t_{0,1}^{\delta_{U}^{j-1}} \cdots t_{0,1}\right)^{\delta_{T}^{i} \delta_{U} \gamma} \\
& =\left(t_{0,1}^{\delta_{U}^{j-1}} \cdots t_{0,1}\right)^{\delta_{T}^{i-1} \delta_{U} \delta_{T} \cdot \Delta\left(t_{0,1}^{-1}\right) \gamma} \\
& =\ldots=\left(t_{U, 1}^{\delta_{U}^{j-1}} \cdots t_{0,1}\right)^{\delta_{U} \delta_{T}^{i} \cdot \Delta\left(t_{0,1}^{-\delta_{T}^{i-1}}\right) \Delta\left(t_{0,1}^{-\delta_{T}^{i-2}}\right) \cdots \Delta\left(t_{0,1}^{-1}\right) \gamma}
\end{aligned}
$$

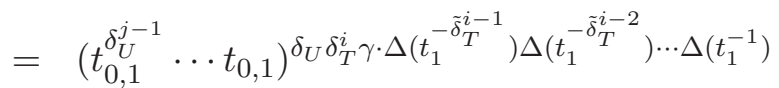

$$
\begin{aligned}
& =\left(t_{0,1}^{\delta_{U}^{j} \delta_{T}^{i} \gamma} \cdots t_{0,1}^{\delta_{U} \delta_{T}^{i} \gamma}\right)^{\Delta\left(t_{1}^{-\tilde{\delta}_{T}^{i-1}}\right) \Delta\left(t_{1}^{-\tilde{\delta}_{T}^{i-2}}\right) \cdots \Delta\left(t_{1}^{-1}\right)}
\end{aligned}
$$

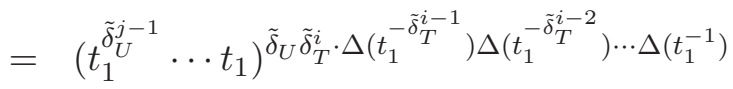

$$
\begin{aligned}
& =\left(t_{1}^{\tilde{\delta}_{U}^{j-1}} \cdots t_{1}\right)^{\tilde{\delta}_{U} \tilde{\delta}_{T} \Delta\left(t_{1}^{-1}\right) \tilde{\delta}_{T}^{i-1} \cdot \Delta\left(t_{1}^{-\tilde{\delta}_{T}^{i-2}}\right) \cdots \Delta\left(t_{1}^{-1}\right)}
\end{aligned}
$$

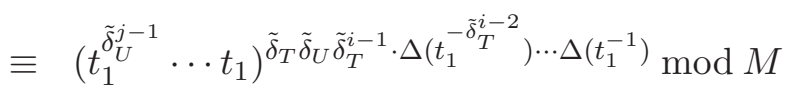

$$
\begin{aligned}
& \equiv \ldots \equiv\left(t_{1}^{\tilde{\delta}_{U}^{j-1}} \cdots t_{1}\right)^{\tilde{\delta}_{T}^{i} \tilde{\delta}_{U}} \bmod M
\end{aligned}
$$

and $\left(t_{1}^{\tilde{\delta}_{U}^{j-1}} \cdots t_{1}\right)^{\tilde{\delta}_{T}^{i} \tilde{\delta}_{U}}=\left(t_{i, j}\right)^{\gamma \tilde{\delta}_{U}}$. Thus $\left(t_{i, j}^{-1} t_{i, j}^{\gamma \chi}\right)^{\delta_{U} \gamma} \in M$. It follows analogously for the other normal generators of $N$ that these are contained in $M$. Thus $F(\mathcal{Z}) / N$ is invariantly finitely $L$-presented and so is our subgroup $H$.

By [70, Theorem 6.1], every finite index subgroup $H$ of an invariantly finitely $L$-presented group $G=\langle\mathcal{X}|\mathcal{Q}| \Phi \mid \mathcal{R}\rangle$ is invariantly finitely $L$-presented whenever the substitutions $\sigma \in \Phi$ induce endomorphisms of the subgroup $H$. This allows us to prove Theorem D using the results in Theorem 5.27 and Theorem 5.26

Proof of Theorem $D \quad$ Let $G$ be a finitely presented group and let $H \unlhd G$ be a finitely generated normal subgroup so that $G / H$ is abelian with torsion-free rank at most two. Since $G$ is finitely generated, $G / H$ is a finitely generated abelian group and so it decomposes into $G / H \cong \mathbb{Z}^{\ell} \times T$ with torsion subgroup $T$ and torsion-free rank $\ell \leq 2$. Denote by $U \leq G$ the full preimage of the torsion subgroup $T$ in $G$. Then $G / U \cong \mathbb{Z}^{\ell}$ and 
$[U: H]<\infty$ hold. If $\ell=0$ holds, $H$ has finite index in $G$ and thus it is invariantly finitely $L$-presented by [70, Theorem 6.1]. If either $\ell=1$ or $\ell=2$ holds, the subgroup $U \unlhd G$ is invariantly finitely $L$-presented by Theorem 5.26 or Theorem 5.27. Each substitution in the $L$-presentation of $U$ is induced by conjugation within the finitely presented group $G$. Since $H$ is a normal subgroup of $G$ each substitution of the finite $L$-presentation of $U$ stabilizes the subgroup $H$. Thus [70, Theorem 6.1] applies to the finite index subgroup $H \unlhd U$ and it shows that $H$ is invariantly finitely $L$-presented.

In the proof of Theorem 5.27, it is essential that the elements $g \in S \cap E K$ have a unique representation in the basis $\left\{t^{i} s^{j} \cdot t_{0,1} \cdot s^{-j} t^{-i} \mid i, j \in \mathbb{Z}\right\}$. This allows us to define the epimorphism $\gamma: F(\mathcal{Y}) \rightarrow F(\mathcal{Z})$ so that it maps conjugates by elements of the Schreier transversal to images of automorphisms which are induced by conjugation with a Schreier transversal. Since $S / S \cap E K$ is finitely presented, we can always choose finitely many Schreier generators $\mathcal{W} \subseteq \mathcal{Y}$ so that $S \cap E K$ is generated, as a normal subgroup, by $\mathcal{W}$. In our proof of Theorem 5.27 the conjugates of these elements in $\mathcal{W}$ by elements of the Schreier transversal from a basis for the subgroup $S \cap E K$. This is no longer possible for $G / H \cong \mathbb{Z} \times \mathbb{Z} \times \mathbb{Z}$ :

Remark 5.28 Consider the notation from the proof of Theorem 5.27. For $G / H \cong$ $\mathbb{Z} \times \mathbb{Z} \times \mathbb{Z}$, we choose as Schreier transversal $\mathcal{T}=\left\{r^{i} s^{j} t^{k} \mid i, j, k \in \mathbb{Z}\right\}$ and we obtain the Schreier generators:

$$
\begin{aligned}
a_{\ell, i, j, k}=\gamma\left(r^{i} s^{j} t^{k}, a_{\ell}\right) & =r^{i} s^{j} t^{k} a_{\ell} t^{-k} s^{-j} r^{-i} \\
s_{i, j, k}=\gamma\left(r^{i} s^{j} t^{k}, s\right) & =r^{i} s^{j}\left(t^{k} s t^{-k} s^{-1}\right) s^{-j} r^{-i} \\
r_{i, j, k}=\gamma\left(r^{i} s^{j} t^{k}, r\right) & =r^{i}\left(s^{j} t^{k} r t^{-k} s^{-j} r^{-1}\right) r^{-i} \\
t_{i, j, k}=\gamma\left(r^{i} s^{j} t^{k}, t\right) & =1
\end{aligned}
$$

where $s_{i, j, k}=1$ if and only if $k=0$ while $r_{i, j, k}=1$ if and only if $(j, k)=(0,0)$. Then

$$
E K=\left\langle a_{\ell, i, j, k}, s_{i, j, o}, r_{i, p, q} \mid 1 \leq \ell \leq m, i, j, k, o, p, q \in \mathbb{Z}, o \neq 0,(p, q) \neq(0,0)\right\rangle
$$

is freely generated and so is

$$
S \cap E K=\left\langle s_{i, j, o}, r_{i, p, q} \mid i, j, o, p, q \in \mathbb{Z}, o \neq 0,(p, q) \neq(0,0)\right\rangle .
$$

Since $G / H \cong S / S \cap E K \cong \mathbb{Z} \times \mathbb{Z} \times \mathbb{Z}$ is finitely presented, the subgroup $S \cap E K$ is finitely generated as a normal subgroup of $S$. In particular, we have that

$$
S / S \cap E K=\langle r, s, t \mid \underbrace{t s t^{-1} s^{-1}}_{=s_{0,0,1}}, \underbrace{t r t^{-1} r^{-1}}_{=r_{0,0,1}}, \underbrace{s r s^{-1} r^{-1}}_{=r_{0,1,0}}\rangle
$$

so that $S \cap E K=\left\langle s_{0,0,1}, r_{0,0,1}, r_{0,1,0}\right\rangle^{S}$ holds. The normal generators of $S \cap E K$ satisfy

$$
\begin{aligned}
& r^{i} s^{j} t^{k} \cdot s_{0,0,1} \cdot t^{-k} s^{-j} r^{-i}=s_{i, j, k+1} \cdot s_{i, j, k}^{-1} \\
& r^{i} s^{j} t^{k} \cdot r_{0,0,1} \cdot t^{-k} s^{-j} r^{-i}=r_{i, j, k+1} \cdot r_{i, j, k}^{-1}, \\
& r^{i} s^{j} t^{k} \cdot r_{0,1,0} \cdot t^{-k} s^{-j} r^{-i}=s_{i, j, k} \cdot r_{i, j+1, k} \cdot s_{i+1, j, k}^{-1} \cdot r_{i, j, k}^{-1} .
\end{aligned}
$$


It can be seen easily (e.g. using GAP) that

$$
U=\left\{s_{i, j, k+1} s_{i, j, k}^{-1}, r_{i, j, k+1} r_{i, j, k}^{-1}, s_{i, j, k} r_{i, j+1, k} s_{i+1, j, k}^{-1} r_{i, j, k}^{-1}\right\}_{i, j, k \in \mathbb{Z}}
$$

is not a basis for $S \cap E K$. Therefore the ideas in the proof of Theorem 5.27 do not apply.

\section{Acknowledgments}

I am grateful to Laurent Bartholdi for valuable comments and suggestions.

René Hartung, Mathematisches Institut, Georg-August Universität Zu Göttingen, Bunsenstrasse 3-5, 37073 Göttingen, Germany

Email: $\quad$ rhartung@uni-math.gwdg.de 
$\Gamma_{\text {Appendix }}$

\title{
Coset Enumeration for Certain Infinitely Presented Groups
}

\begin{abstract}
We describe an algorithm that computes the index of a finitely generated subgroup in a finitely $L$-presented group provided that this index is finite. This algorithm shows that the subgroup membership problem for finite index subgroups in a finitely $L$-presented group is decidable. As an application, we consider the low-index subgroups of some self-similar groups including the Grigorchuk group, the twisted twin of the Grigorchuk group, the Grigorchuk super-group, and the Hanoi 3-group.
\end{abstract}

Keywords. Coset enumeration; recursive presentations; self-similar groups; Grigorchuk group; low-index subgroups.

\section{C.1 Introduction}

Many algorithmic problems are unsolvable for finitely presented groups in general. For instance, there is no algorithm which allows to decide if a group given by a finite presentation is trivial [95]. However, the coset enumeration process introduced by Todd \& Coxeter [133] and investigated by various others, see [76] or the historical notes in Chapter 5.9 of [129], computes the index of a finitely generated subgroup in a finitely presented group provided that this index is finite. Therefore, the Todd-Coxeter method allows one to prove that a finitely presented group is trivial.

Coset enumeration is one of the most important tools for investigating finitely presented groups; but, if the subgroup has infinite index, this process will not terminate. Even if the subgroup has finite index, there is no upper bound on the complexity of coset enumeration. Therefore, even proving a finitely presented group being trivial is computationally a challenging problem [76, 104]. 
For this reason, solving algorithmic problems for infinitely presented groups seems entirely infeasible. However, an interesting family of recursively presented groups was recently shown to be applicable for computer investigations. Examples of such groups arise as subgroups of the automorphism group of a regular tree. A famous example is the Grigorchuk group $\mathfrak{G}$ which plays a prominent role in the area of Burnside problems [53]. The group $\mathfrak{G}$ is finitely generated and it admits a recursive presentation whose relations are given recursively by the action of a finitely generated free monoid of endomorphisms acting on finitely many relations [96]. Infinite presentations of this type are called finite L-presentations in honor of Lysënok's latter result for the Grigorchuk group $\mathfrak{G}$; see Section C.2 or [6] for a definition.

Finite $L$-presentations are 'natural' generalizations of finite presentations and, as the concept is quite general, they found their application in various aspects of group theory; see, for instance, [18, 85]. A finite $L$-presentation of a group allows to compute its lower central series quotients [9] and the Dwyer quotients of its Schur multiplier [66]. The Dwyer quotients often exhibit periodicities which yield detailed information on the structure of the Schur multiplier in general.

In this paper, we describe a coset enumeration process for computing the index of a finitely generated subgroup in a finitely $L$-presented group provided that this index is finite. In order to achieve this method, we show in Section C.3 that finitely many relations are sufficient to compute an upper bound on the index using coset enumeration for finitely presented groups. It then remains to either prove that this upper bound is sharp or to improve the bound otherwise. In Section C.4 we show that the latter problem is algorithmically decidable in general. In particular, we show that there exists an algorithm which decides whether or not a map from the free group over the $L$-presentations generators into a finite group induces a homomorphism from the $L$-presented group.

Similar to coset enumeration for finitely presented groups, our method for finitely $L$-presented groups allows straightforward applications including a membership test for finite index subgroups. In particular, our method allows us to compute the number of subgroups with small index for some self-similar groups in Section C.5. Our explicit computations correct the counts obtained in [11,12], and hence we provide a further step towards Problem 6.1 raised in 58].

We have implemented our coset enumeration method and its applications in the computer algebra system GAP [50]. Computer experiments with this implementation demonstrate that our method works reasonably well in practice.

In a forthcoming paper, we prove a variant of the Reidemeister-Schreier theorem for finitely $L$-presented groups which shows that each finite index subgroup of a finitely $L$-presented group is finitely $L$-presented itself.

\section{C.2 Preliminaries}

We briefly recall the notion of a finite $L$-presentation as introduced in [6]. For this purpose, let $F$ be a finitely generated free group over the alphabet $\mathcal{X}$. Furthermore, let 
$\mathcal{Q}, \mathcal{R} \subset F$ and $\Phi \subset \operatorname{End}(F)$ be finite subsets. Then the quadruple $\langle\mathcal{X}|\mathcal{Q}| \Phi \mid \mathcal{R}\rangle$ is a finite L-presentation. It defines the finitely L-presented group

$$
G=\left\langle\mathcal{X} \mid \mathcal{Q} \cup \bigcup_{\sigma \in \Phi^{*}} \mathcal{R}^{\sigma}\right\rangle
$$

where $\Phi^{*}$ denotes the free monoid of endomorphisms generated by $\Phi$; that is, the closure of $\{$ id $\} \cup \Phi$ under composition of endomorphisms. We will also write $G=\langle\mathcal{X}|\mathcal{Q}| \Phi \mid \mathcal{R}\rangle$ for the finitely $L$-presented group in Eq. (C.1).

Clearly, every finitely presented group $\langle\mathcal{X} \mid \mathcal{R}\rangle$ is finitely $L$-presented by $\langle\mathcal{X}|\emptyset|$ $\emptyset|\mathcal{R}\rangle$. Therefore, finite $L$-presentations generalize the concept of finite presentations. Other examples of finitely $L$-presented groups are various self-similar groups or branch groups [6]. For instance, the Grigorchuk group satisfies the following

Theorem 2.1 (Lysënok, 1985) The Grigorchuk group $\mathfrak{G}$ is finitely L-presented by

$$
\left\langle\{a, b, c, d\}\left|\left\{a^{2}, b^{2}, c^{2}, d^{2}, b c d\right\}\right|\{\sigma\} \mid\left\{(a d)^{4},(a d a c a c)^{4}\right\}\right\rangle,
$$

where $\sigma$ is the endomorphism of the free group over the alphabet $\{a, b, c, d\}$ induced by the mapping $a \mapsto a c a, b \mapsto d, c \mapsto b$, and $d \mapsto c$.

Proof. For a proof, we refer to [96].

Finite $L$-presentations are recursive presentations which are 'natural' generalizations of finite presentations. They were used by various authors to construct groups with interesting properties; see, for instance, [18, 85. Furthermore, every free group in a variety of groups that satisfies finitely many identities is finitely $L$-presented [6]; e.g., the free Burnside group $B(n, m)$ of exponent $m$ on $n$ generators is finitely $L$-presented by

$$
\left\langle\left\{a_{1}, \ldots, a_{n}\right\} \cup\{t\}|\{t\}| \Sigma \mid\left\{t^{m}\right\}\right\rangle,
$$

where the endomorphisms $\Sigma=\left\{\sigma_{x} \mid x \in\left\{a_{1}^{ \pm 1}, \ldots, a_{n}^{ \pm 1}\right\}\right\}$ are induced by the mappings

$$
\sigma_{x}:\left\{\begin{aligned}
a_{i} & \mapsto a_{i}, \quad \text { for each } 1 \leq i \leq n \\
t & \mapsto t x,
\end{aligned}\right.
$$

for each $x \in\left\{a_{1}^{ \pm 1}, \ldots, a_{n}^{ \pm 1}\right\}$.

\section{C.3 Coset Enumeration for Finitely $L$-Presented Groups}

Let $G=\langle\mathcal{X}|\mathcal{Q}| \Phi \mid \mathcal{R}\rangle$ be a finitely $L$-presented group and let $\mathcal{U} \leq G$ be a finitely generated subgroup with finite index in $G$. In this section, we show that coset enumeration for finitely presented groups yields an upper bound on the index $[G: \mathcal{U}]$. In Section C.4. it then remains to prove (or disprove) that this upper bound is sharp. 
Let $\left\{g_{1}, \ldots, g_{n}\right\}$ be a generating set for the subgroup $\mathcal{U}$. We assume that the generators of $\mathcal{U}$ are given as words over the alphabet $\mathcal{X} \cup \mathcal{X}^{-}$. Denote the free group over $\mathcal{X}$ by $F$ and let $K$ be the normal subgroup

$$
K=\left\langle\mathcal{Q} \cup \bigcup_{\sigma \in \Phi^{*}} \mathcal{R}^{\sigma}\right\rangle^{F}
$$

so that $G \cong F / K$ holds. Then the subgroup $E=\left\langle g_{1}, \ldots, g_{n}\right\rangle \leq F$ satisfies that $\mathcal{U} \cong$ $E K / K$. Hence, we are to compute the index $[G: \mathcal{U}]=[F: E K]$.

For an element $\sigma \in \Phi^{*}$, we denote by $\|\sigma\|$ the usual word-length in the generating set $\Phi$ of the free monoid $\Phi^{*}$. Define $\Phi^{(i)}=\left\{\sigma \in \Phi^{*} \mid\|\sigma\| \leq i\right\}$, for each $i \in \mathbb{N}_{0}$. Then, as $\mathcal{Q}$, $\Phi$, and $\mathcal{R}$ are finite sets, the normal subgroup

$$
K_{i}=\left\langle\mathcal{Q} \cup \bigcup_{\sigma \in \Phi^{(i)}} \mathcal{R}^{\sigma}\right\rangle^{F}
$$

is finitely generated as normal subgroup. We obtain $K=\bigcup_{i \geq 0} K_{i}$ and also $E K=$ $\bigcup_{i \geq 0} E K_{i}$. Consider the ascending chain of subgroups

$$
E K_{0} \leq E K_{1} \leq \ldots \leq E K_{\ell} \leq E K_{\ell+1} \leq \ldots \leq E K \leq F .
$$

Then the following lemma is straightforward.

Lemma 3.2 The subgroup EK has finite index in $F$ if and only if there exists $\ell \in \mathbb{N}$ such that $E K_{\ell}$ has finite index in $F$. In that case, there exists $\ell^{\prime} \in \mathbb{N}$ such that $E K_{\ell^{\prime}}=E K$.

Proof. Obviously, if $\left[F: E K_{\ell}\right]$ is finite for some $\ell \in \mathbb{N}$, then the subgroup $E K$ has finite index in $F$. On the other hand, if $[F: E K]$ is finite, then, as $F$ is finitely generated, the subgroup $E K$ is finitely generated. Let $\left\{u_{1}, \ldots, u_{n}\right\}$ be a generating set of $E K$. Since $E K=\bigcup_{i>0} E K_{i}$ holds, there exists a positive integer $\ell \in \mathbb{N}$ such that $\left\{u_{1}, \ldots, u_{n}\right\} \subseteq E K_{\ell}$ and thus $E K_{\ell}=E K$.

Note that the index $\left[F: E K_{\ell}\right]$ is the index of the subgroup $\mathcal{U}$ in the finitely presented covering group

$$
G_{\ell}=\left\langle\mathcal{X} \mid\left\{q, r^{\sigma} \mid q \in \mathcal{Q}, r \in \mathcal{R}, \sigma \in \Phi^{(\ell)}\right\}\right\rangle .
$$

By Lemma 3.2, there exists a positive integer $\ell \in \mathbb{N}$ so that the subgroup $\mathcal{U}$ has finite index in $G_{\ell}$. In this case, coset enumeration for finitely presented groups computes the index $\left[G_{\ell}: \mathcal{U}\right]$. Although we do not know this integer $\ell$ a priori, we can use the following firsthand approach to find such an integer: Starting with $\ell=1$, we attempt to prove finiteness of $\left[G_{\ell}: \mathcal{U}\right]$ using coset enumeration for finitely presented groups. If this attempt does not succeed within a previously defined time limit, we increase the integer $\ell$ and the time limit. We continue this process until eventually the index $\left[G_{\ell}: \mathcal{U}\right]$ is proved to be finite. In theory, Lemma 3.2 guarantees that this process will terminate. Computer experiments with the implementation of our method in GAP show that this firsthand approach works reasonably well in practice. In particular, our implementation allows to compute the index of all subgroups considered in [7, 11, 58] and Chapter VIII of [38]. 
Suppose that the integer $\ell \in \mathbb{N}$ is chosen so that $n=\left[G_{\ell}: \mathcal{U}\right]$ is finite and that the coset enumeration for finitely presented groups has terminated and has computed a permutation representation $\varphi_{\ell}: F \rightarrow \mathcal{S}_{n}$ for the group's action on the right cosets $E K_{\ell} \backslash F$. Then the index $[G: \mathcal{U}]=[F: E K]$ divides the index $\left[G_{\ell}: \mathcal{U}\right]=\left[F: E K_{\ell}\right]$, and hence $\left[G_{\ell}: \mathcal{U}\right]$ is an upper bound on $[G: \mathcal{U}]$. It therefore remains to either prove that $[F: E K]=\left[F: E K_{\ell}\right]$ holds, or to increase the integer $\ell$ otherwise. The permutation representation $\varphi_{\ell}: F \rightarrow \mathcal{S}_{n}$ is called valid, if $[F: E K]=\left[F: E K_{\ell}\right]$ holds.

Clearly, a permutation representation $\varphi_{\ell}: F \rightarrow \mathcal{S}_{n}$ is valid if and only if every relation $r \in F$ of the group presentation is contained in the kernel of $\varphi_{\ell}$. Therefore, if the group $G=F / K$ were finitely presented, only finitely many relations need to be considered to prove validity of $\varphi_{\ell}$. However, for finitely $L$-presented groups, even checking validity of a permutation representation $\varphi_{\ell}$ involves possibly infinitely many relations. In Section C.4. we prove that the latter problem is decidable in general.

\section{C.4 Deciding Validity of a Permutation Representation}

In this section, we describe our algorithm for deciding whether or not a permutation representation $\varphi: F \rightarrow \mathcal{S}_{n}$, as considered in Section C.3, is valid. This is equivalent to checking whether a coset-table for $\mathcal{U}$ in $G_{\ell}$ obtained by the methods of Section C.3 defines the given subgroup $\mathcal{U} \leq G$.

Let $\varphi: F \rightarrow \mathcal{S}_{n}$ be a permutation representation as in Section C.3 and let $\Phi^{*}$ be the free monoid generated by a finite set $\Phi \subseteq \operatorname{End}(F)$. For two endomorphisms $\sigma \in \Phi^{*}$ and $\delta \in \Phi^{*}$, we say that $\delta$ reduces to $\sigma$ with respect to $\varphi$ if there exists a homomorphism $\pi: \operatorname{im}(\sigma \varphi) \rightarrow \operatorname{im}(\delta \varphi)$ such that $\sigma \varphi \pi=\delta \varphi$. In this case, we will write $\delta \sim \varphi \sigma$. Note that $\leadsto$ is a reflexive and transitive relation on the endomorphisms $\Phi^{*}$. The following lemma gives an equivalent definition for $\delta \leadsto \varphi \sigma$.

Lemma 4.3 Let $\delta, \sigma \in \operatorname{End}(F)$ be given. Then $\delta$ reduces to $\sigma$ with respect to $\varphi$ if and only if $\operatorname{ker}(\sigma \varphi) \leq \operatorname{ker}(\delta \varphi)$ holds.

Proof. Assume that $\delta \sim \varphi \sigma$ holds. Then, by definition, there exists a homomorphism $\pi: \operatorname{im}(\sigma \varphi) \rightarrow \operatorname{im}(\delta \varphi)$ such that $\sigma \varphi \pi=\delta \varphi$. Let $g \in \operatorname{ker}(\sigma \varphi)$. Then we have that $g^{\delta \varphi}=$ $g^{\sigma \varphi \pi}=\left(g^{\sigma \varphi}\right)^{\pi}=1$ and hence, we obtain $g \in \operatorname{ker}(\delta \varphi)$. Suppose that $\operatorname{ker}(\sigma \varphi) \leq \operatorname{ker}(\delta \varphi)$ holds. Then we have the isomorphisms $F / \operatorname{ker}(\sigma \varphi) \rightarrow \operatorname{im}(\sigma \varphi), g \operatorname{ker}(\sigma \varphi) \mapsto g^{\sigma \varphi}$ and $F / \operatorname{ker}(\delta \varphi) \rightarrow \operatorname{im}(\delta \varphi), g \operatorname{ker}(\delta \varphi) \mapsto g^{\delta \varphi}$. We further have the natural homomorphism $F / \operatorname{ker}(\sigma \varphi) \rightarrow F / \operatorname{ker}(\delta \varphi), g \operatorname{ker}(\sigma \varphi) \mapsto g \operatorname{ker}(\delta \varphi)$. This yields the existence of a homomorphism $\pi: \operatorname{im}(\sigma \varphi) \rightarrow \operatorname{im}(\delta \varphi)$ such that $g^{\sigma \varphi \pi}=g^{\delta \varphi}$.

A finite generating set for the kernel $\operatorname{ker}(\sigma \varphi)$ is given by the Schreier theorem [95, Proposition 3.7] and hence, it is straightforward to check whether or not $\delta \leadsto_{\varphi} \sigma$ holds. The definition $\delta \hookrightarrow_{\varphi} \sigma$ also yields the following immediate consequence.

Lemma 4.4 There is no infinite set of endomorphisms of $F$ such that for each pair $(\sigma, \delta)$ from this set, neither $\sigma \sim_{\varphi} \delta$ nor $\delta \sim_{\varphi} \sigma$ hold. 
Proof. Obviously, for every endomorphism $\sigma \in \operatorname{End}(F)$, it holds that $\sigma \sim_{\varphi} \sigma$. By the universal property of the free group $F$, a homomorphism $\sigma \varphi: F \rightarrow \mathcal{S}_{n}$ is uniquely defined by the images $x_{1}^{\sigma \varphi}, \ldots, x_{n}^{\sigma \varphi}$ of the elements $x_{1}, \ldots, x_{n}$ of a basis of $F$. Since $\operatorname{im}(\varphi)$ is a finite group, there are only finitely many homomorphisms $F \rightarrow \operatorname{im}(\varphi)$ and therefore $\operatorname{Hom}(F, \operatorname{im}(\varphi))$ is finite. Hence, an infinite set of endomorphisms will contain endomorphisms $\sigma$ and $\delta$ with $x_{i}^{\sigma \varphi}=x_{i}^{\delta \varphi}$, for each $1 \leq i \leq n$. In this case, $\sigma \leadsto_{\varphi} \delta$ obviously holds.

An element $\sigma \in \Phi^{*}$ is called a $\Phi$-descendant of $\delta \in \Phi^{*}$ if there exists $\psi \in \Phi$ such that $\sigma=\psi \delta$. Thereby, the free monoid $\Phi^{*}$ obtains the structure of a $|\Phi|$-regular rooted tree with its root being the identity map id: $F \rightarrow F$. We can further endow the monoid $\Phi^{*}$ with a length-plus-(from the right)-lexicographic ordering $\prec$ by choosing an arbitrary ordering on the finite set $\Phi$. More precisely, we define $\sigma \prec \delta$ if $\|\sigma\|<\|\delta\|$ or, otherwise, if $\sigma=\sigma_{1} \cdots \sigma_{n}$ and $\delta=\delta_{1} \cdots \delta_{n}$, with $\sigma_{i}, \delta_{i} \in \Phi$, and there exists a positive integer $1 \leq k \leq n$ such that $\sigma_{i}=\delta_{i}$, for $k<i \leq n$, and $\sigma_{k} \prec \delta_{k}$. Since $\Phi$ is finite, the obtained ordering $\prec$ is a well-ordering on the monoid $\Phi^{*}$, see [129], and therefore there is no infinite $\prec$-descending series of endomorphisms in $\Phi^{*}$.

Our algorithm that decides validity of a permutation representation $\varphi: F \rightarrow \mathcal{S}_{n}$ is displayed in Algorithm C.1 below. We need to prove the following

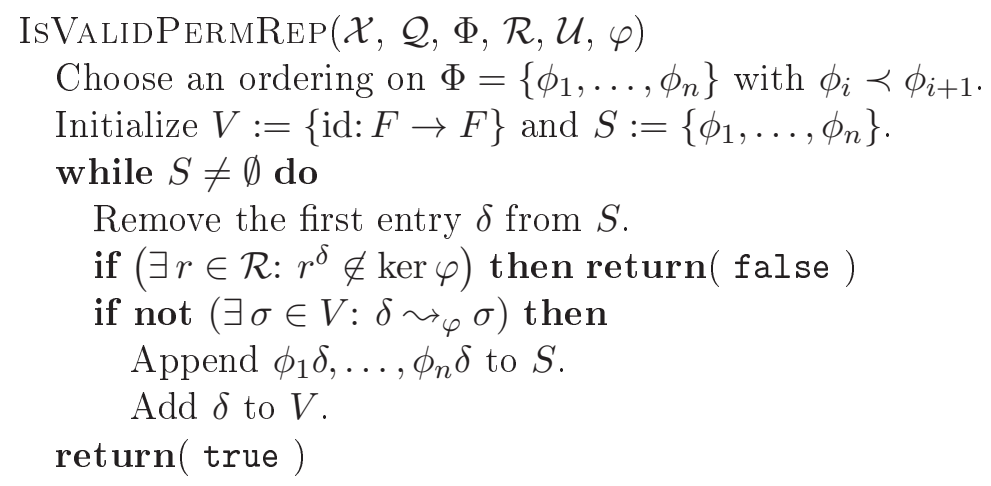

Algorithm C.1: Deciding validity of a permutation representation

Theorem 4.5 The algorithm IsVALIDPERMREP returns true if and only if the permutation representation $\varphi: F \rightarrow \mathcal{S}_{n}$ is valid.

Proof. The ordering $\prec$ on $\Phi$ can be extended to an ordering on $\Phi^{*}$ as described above. By construction, the stack $S$ is ordered with respect to $\prec$. Since $F$ is finitely generated, the set of homomorphisms $\operatorname{Hom}\left(F, \mathcal{S}_{n}\right)$ is finite. Thus, in particular, the set $\{\delta \varphi \mid \delta \in$ $V\} \subseteq \operatorname{Hom}\left(F, \mathcal{S}_{n}\right)$ is finite and therefore the algorithm IsVALidPermReP can add only finitely many endomorphisms to the set $V$. Thus, for every $\Phi$-descendant $\delta$ in the stack $S$, there will eventually exist an element $\sigma \in V$ such that $\delta \leadsto_{\varphi} \sigma$. Therefore, the algorithm IsVALIDPERMREP is guaranteed to terminate and it returns either true or 
false. Clearly, if the algorithm returned false, then it found a relation $r^{\delta}$ which yields a coincidence, and hence the permutation representation $\varphi: F \rightarrow \mathcal{S}_{n}$ is not valid.

Suppose that the algorithm returned true. By the constructions of Section C.3, the fixed relations in $\mathcal{Q}$ of the $L$-presentation $\langle\mathcal{X}|\mathcal{Q}| \Phi \mid \mathcal{R}\rangle$ are already contained in the kernel of the permutation representation $\varphi$. Therefore, it suffices to prove that every relation of the form $r^{\sigma_{1}}$, with $r \in \mathcal{R}$ and $\sigma_{1} \in \Phi^{*}$, is contained in the kernel of $\varphi$. By construction, there exists $\delta \in V$ maximal subject to the existence of $w \in \Phi^{*}$ such that $\sigma_{1}=w \delta$. If $\|w\|=0$, then $\sigma_{1}=\delta$ is contained in $V$ and therefore $r^{\delta} \in \operatorname{ker} \varphi$, as the algorithm did not return false. Otherwise, there exist $\psi \in \Phi$ and $v \in \Phi^{*}$ such that $w \delta=v \psi \delta$. Since $\psi \delta \notin V$, there exists an element $\varepsilon \in V$ with $\varepsilon \prec \psi \delta$, by construction, such that $\psi \delta$ reduces to $\varepsilon$ with respect to $\varphi$. Thus, by definition, there exists a homomorphism $\pi: \operatorname{im}(\varepsilon \varphi) \rightarrow \operatorname{im}(\psi \delta \varphi)$ such that $\psi \delta \varphi=\varepsilon \varphi \pi$. In particular, we obtain that $r^{\sigma_{1} \varphi}=r^{w \delta \varphi}=r^{v \psi \delta \varphi}=r^{v \varepsilon \varphi \pi}$. As $\pi$ is a homomorphism, it suffices to prove that $r^{v \varepsilon} \in \operatorname{ker} \varphi$. Note that, since $\varepsilon \prec \psi \delta$, we have that $v \varepsilon \prec v \psi \delta=\sigma_{1}$. Continuing this rewriting process with the element $\sigma_{2}=v \varepsilon$ yields a descending sequence $\sigma_{1} \succ \sigma_{2} \succ \ldots$ in the monoid $\Phi^{*}$. As the ordering $\prec$ is a well-ordering, this process terminates with an element $\sigma_{n} \in V$. Since the algorithm did not return false, we have that $r^{\sigma_{n}} \in \operatorname{ker} \varphi$ which proves the assertion.

Note that, if the algorithm IsVALidPERMReP found a coincidence, this can be used to update the coset-table, and thus another application of coset enumeration for finitely presented groups can be avoided. Moreover, the Algorithm C.1 yields the following

Theorem 4.6 Let $G$ be finitely L-presented by $\langle\mathcal{X}|\mathcal{Q}| \Phi \mid \mathcal{R}\rangle$ and denote the free group over $\mathcal{X}$ by $F$. There exists an algorithm which decides whether or not a homomorphism $\varphi: F \rightarrow \mathcal{S}_{n}$ induces a homomorphism $G \rightarrow \mathcal{S}_{n}$.

If $\Phi^{*}=\{\sigma\}^{*}$ is generated by a single element $\sigma \in \operatorname{End}(F)$, then there will exist positive integers $0 \leq i<j$ such that $\sigma^{j} \leadsto \varphi \sigma^{i}$. In this case, the algorithm IsVALIDPERmREP simplifies to the following

Corollary 4.7 Let $0 \leq i<j$ be positive integers such that $\sigma^{j} \sim_{\varphi} \sigma^{i}$. Then we have $\left[F: E K_{\ell}\right]=[F: E K]$ if and only if

$$
\left\{q, r^{\sigma^{k}} \mid q \in \mathcal{Q}, r \in \mathcal{R}, 0 \leq k<j\right\} \subseteq \operatorname{ker} \varphi .
$$

We consider the following

Example 4.8 Let $G$ denote the Basilica Group [60]. Then $G$ is finitely L-presented by $\left\langle\{a, b\}|\emptyset|\{\sigma\} \mid\left\{\left[a, a^{b}\right]\right\}\right\rangle$, where $\sigma$ is induced by the mapping $a \mapsto b^{2}$ and $b \mapsto a$; see [16]. We consider the subgroup $\mathcal{U}=\left\langle a^{3}, b, a b a\right\rangle$. A coset enumeration for finitely presented groups yields that the subgroup $\mathcal{U}$ has index 3 in the finitely presented covering group

$$
G_{0}=\left\langle\{a, b\} \mid\left\{\left[a, a^{b}\right]\right\}\right\rangle .
$$

Furthermore, we obtain the permutation representation $\varphi: F \rightarrow \mathcal{S}_{3}$ for the group's action on the cosets $E K_{0} \backslash F$. This permutation representation is induced by the mapping

$$
a \mapsto(1,2,3) \quad \text { and } \quad b \mapsto(2,3) .
$$


We now obtain the images

$$
\begin{aligned}
a^{\sigma \varphi} & =(), & b^{\sigma \varphi} & =(1,2,3), \\
a^{\sigma^{2} \varphi} & =(1,3,2), & b^{\sigma^{2} \varphi} & =(), \\
a^{\sigma^{3} \varphi} & =(), & b^{\sigma^{3} \varphi} & =(1,3,2) .
\end{aligned}
$$

Clearly, the mapping $a^{\sigma \varphi} \mapsto a^{\sigma^{3} \varphi}$ and $b^{\sigma \varphi} \mapsto b^{\sigma^{3} \varphi}$ induces a homomorphism $\pi: \operatorname{im}(\sigma \varphi) \rightarrow$ $\operatorname{im}\left(\sigma^{3} \varphi\right)$, and hence we have $\sigma^{3} \leadsto \varphi \sigma$. By Corollary 4.7, it therefore suffices to prove that

$$
\left(\left[a, a^{b}\right]\right)^{\varphi}=(), \quad\left(\left[a, a^{b}\right]\right)^{\sigma \varphi}=(), \quad \text { and } \quad\left(\left[a, a^{b}\right]\right)^{\sigma^{2} \varphi}=()
$$

hold. This yields that $[G: \mathcal{U}]=3$.

\section{C.5 Further Applications}

The permutation representation $\varphi: F \rightarrow \mathcal{S}_{n}$ for a finite index subgroup $E K / K \leq F / K$ yields various algorithmic applications. For instance, an element $w \in F$ is contained in the given subgroup $E K$ if and only if it stabilizes the trivial coset $E K 1$. This can be easily be checked using the permutation representation $\varphi$. In particular, we obtain

Theorem 5.9 The subgroup membership problem for finite index subgroups in a finitely $L$-presented group is decidable.

Moreover, having computed permutation representations $\varphi_{1}$ and $\varphi_{2}$ for two finite index subgroups $U$ and $V$ of a finitely $L$-presented group, one can compute a generating set for the intersection $U \cap V$. Thus, in particular, our method allows one to compute the core of a finite index subgroup. For example, the core of the subgroup $\mathcal{U}$ in Example 4.8 is given by

$$
H=\left\langle b^{2}, a^{3}, a^{2} b a^{-1} b^{-1}, a b a b^{-1}, a b^{2} a^{-1}, b a^{2} b^{-1} a^{-1}, b a b a^{-2}\right\rangle .
$$

Since $H$ has finite index in $G$, our method allows to compute a permutation representation for the core $H$ and we obtain $G / H \cong \mathcal{S}_{3}$.

\section{Low-Index Subgroups of Finitely $L$-Presented Groups}

The coset enumeration process for finitely presented groups was used in [40] to describe a low-index subgroup algorithm that computes all subgroups of a finitely presented group up to a given index. This algorithm also yields a method for computing all subgroups with small index in a finitely $L$-presented group. In this section, we will describe this method for finitely $L$-presented groups and we use this algorithm to investigate some selfsimilar groups. In particular, our implementation in the computer algebra system GAP allows us to determine the number of subgroups with index at most 64 in the Grigorchuk group.

Let $G=F / K$ be a finitely $L$-presented group and let $n$ be a positive integer. Using the low-index subgroup algorithm for finitely presented groups [40], see also Chapter 5.6 
of [129], we obtain the list of subgroups with index at most $n$ in the finitely presented covering group $G_{\ell}=F / K_{\ell}$. Since the covering group $G_{\ell}$ naturally maps onto $G$, every subgroup $E K_{\ell} / K_{\ell}$ with index $n$ in $G_{\ell}$ maps to a subgroup of the finitely $L$-presented group $G$. The index of this image $E K / K$ in $G$ divides the index $n=\left[F: E K_{\ell}\right]$. On the other hand, every subgroup $E K / K$ with index $n$ in the finitely $L$-presented group $F / K$ has a full preimage $E K / K_{\ell}$ in the finitely presented group $G_{\ell}$ with index $n$. Thus the list of subgroups with index at most $n$ in a finitely $L$-presented group $G$ can be obtained from the list of subgroups of a finitely presented covering group $G_{\ell}$ by removing duplicate images. Our solution to the subgroup membership problem can be used to remove duplicate images in $G$.

As an application, we consider some interesting self-similar groups and we determine the number of subgroups with small index. We first consider the Grigorchuk group $\mathfrak{G}$ : its lattice of normal subgroups is well-understood [7] while its lattice of subgroups with finite index is widely unknown [58]. It is well known [58] that the Grigorchuk group has seven subgroups of index two. In [116], it was shown that the subgroups of index two are the only maximal subgroups of $\mathfrak{G}$. Our low-index subgroup algorithm allows us to determine the number of subgroups with index at most 64 in the group $\mathfrak{G}$ and thereby, it corrects the counts in Section 7.4 of [12] and in Section 4.1 of [11. The following list summarizes the number of subgroups $(\leq)$ and the number of normal subgroups $(\unlhd)$ among them:

\begin{tabular}{cccccccc}
\hline index & 1 & 2 & 4 & 8 & 16 & 32 & 64 \\
\hline$\leq$ & 1 & 7 & 31 & 183 & 1827 & 22931 & 378403 \\
$\unlhd$ & 1 & 7 & 7 & 7 & 5 & 3 & 3 \\
\hline
\end{tabular}

The Grigorchuk super-group $\tilde{\mathfrak{G}}$ was introduced in [11]. It contains the Grigorchuk group as an infinite index subgroup. Little is known about its subgroup lattice. The twisted twin $\overline{\mathfrak{G}}$ of the Grigorchuk group was introduced in [14]. Similarly, little is known about the subgroup lattice of the twisted twin $\overline{\mathfrak{G}}$. Our low-index subgroup algorithm allows us to determine the number of subgroups with index at most 16 in both groups. Their subgroup counts are:

\begin{tabular}{crrrr}
\hline index & \multicolumn{2}{c}{$\leq^{\tilde{\mathfrak{G}}}$} & \multicolumn{2}{c}{$\overline{\mathfrak{G}}$} \\
& \multicolumn{1}{c}{$\leq$} & \multicolumn{1}{c}{$\leq$} & $\unlhd$ \\
\hline 1 & 1 & 1 & 1 & 1 \\
2 & 15 & 15 & 15 & 15 \\
4 & 147 & 35 & 147 & 35 \\
8 & 2163 & 43 & 1963 & 43 \\
16 & 52403 & 55 & 46723 & 47 \\
\hline
\end{tabular}

As both groups are 2-groups, the only maximal subgroups with finite index are the subgroups with index two; though the question of determining all maximal subgroups of $\tilde{\mathfrak{G}}$ and $\overline{\mathfrak{G}}$ has not been addressed in this paper.

Finally, we consider the Basilica group and the Hanoi-3 group [59] with its $L$ presentation from [15]. The following list also includes the number of maximal subgroups 
$(\max )$ :

\begin{tabular}{|c|c|c|c|c|c|c|}
\hline \multirow{2}{*}{ index } & \multicolumn{3}{|c|}{ Hanoi-3 } & \multicolumn{3}{|c|}{ Basilica } \\
\hline & $\leq$ & $\unlhd$ & $\max$ & $\leq$ & $\unlhd$ & $\max$ \\
\hline 1 & 1 & 1 & 1 & 1 & 1 & 1 \\
\hline 2 & 7 & 7 & 7 & 3 & 3 & 3 \\
\hline 3 & 12 & 0 & 12 & 7 & 4 & 7 \\
\hline 4 & 59 & 7 & 4 & 19 & 7 & 0 \\
\hline 5 & 15 & 0 & 15 & 11 & 6 & 11 \\
\hline 6 & 136 & 4 & 0 & 39 & 13 & 0 \\
\hline 7 & 21 & 0 & 21 & 15 & 8 & 15 \\
\hline 8 & 335 & 13 & 0 & 163 & 19 & 0 \\
\hline 9 & 225 & 0 & 0 & 115 & 13 & 9 \\
\hline 10 & 153 & 3 & 0 & 83 & 19 & 0 \\
\hline 11 & 33 & 0 & 33 & 23 & 12 & 23 \\
\hline 12 & 2872 & 12 & 0 & 355 & 31 & 0 \\
\hline 13 & 39 & 0 & 39 & 27 & 14 & 27 \\
\hline 14 & 297 & 3 & 0 & 115 & 25 & 0 \\
\hline 15 & 450 & 0 & 0 & 77 & 24 & 0 \\
\hline 16 & 1855 & 13 & 0 & 1843 & 47 & 0 \\
\hline 17 & 51 & 0 & 51 & 35 & 18 & 35 \\
\hline 18 & 5001 & 3 & 0 & 1047 & 44 & 0 \\
\hline 19 & 57 & 0 & 57 & 39 & 20 & 39 \\
\hline 20 & 1189 & 9 & 0 & 939 & 45 & 0 \\
\hline 21 & 756 & 0 & 0 & 105 & 32 & 0 \\
\hline 22 & 531 & 3 & 0 & 223 & 37 & 0 \\
\hline 23 & 69 & 0 & 69 & 47 & 24 & 47 \\
\hline 24 & 52220 & 23 & 0 & 4723 & 87 & 0 \\
\hline 25 & 225 & 0 & 75 & 411 & 31 & 25 \\
\hline 26 & 783 & 3 & 0 & 315 & 43 & 0 \\
\hline 27 & 5616 & 0 & 27 & 736 & 49 & 0 \\
\hline 28 & 2301 & 9 & 0 & & & \\
\hline 29 & 87 & 0 & 87 & & & \\
\hline 30 & 15462 & 3 & 0 & & & \\
\hline 31 & 93 & 0 & 93 & & & \\
\hline 32 & 9119 & 25 & 0 & & & \\
\hline
\end{tabular}

The largest abelian quotient $H / H^{\prime}$ of the Hanoi-3 group $H$ is 2-elementary abelian of rank 3. Thus, by the Feit-Thompson theorem [47], there are no normal subgroups with odd index in the Hanoi-3 group.

\section{Acknowledgments}

I am grateful to Laurent Bartholdi for valuable comments and suggestions. 
René Hartung, Mathematisches Institut, Georg-August Universität Zu Göttingen, Bunsenstrasse 3-5, 37073 Göttingen, Germany

Email: $\quad$ rhartung@uni-math.gwdg.de

June 2010 (revised May 2011). 


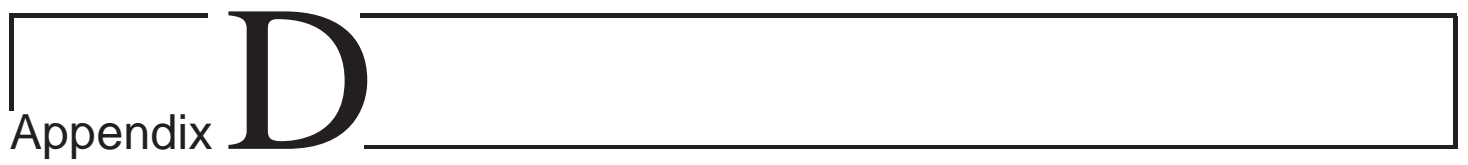

\title{
A Reidemeister-Schreier Theorem for Finitely $L$-Presented Groups
}

\begin{abstract}
We prove a variant of the well-known Reidemeister-Schreier Theorem for finitely $L$-presented groups. More precisely, we prove that each finite index subgroup of a finitely $L$-presented group is itself finitely $L$-presented. Our proof is constructive and it yields a finite $L$-presentation for the subgroup. We further study conditions on a finite index subgroup of an invariantly finitely $L$-presented group to be invariantly $L$-presented itself.
\end{abstract}

Keywords. Reidemeister-Schreier Theorem; infinite presentations; recursive presentations; self-similar groups; Basilica group; Grigorchuk group; finite index subgroups;

Mathematics Subject Classification 2010: 20F05, 20E07, 20-04

\section{D.1 Introduction}

Group presentations play an important role in computational group theory. In particular finite presentations have been subject to extensive research in computational group theory dating back to the early days of computer-algebra-systems [32, 88, 102, 129]. Group presentations, on the one hand, provide an effective description of the group. On the other hand, a description of a group by its generators and relations leads to various decision problems which are known to be unsolvable in general [95]. For instance, the word problem of a finitely presented group is unsolvable [27,113]. However, various total and partial algorithms for finitely presented groups are known [129]. For instance, the coset-enumeration process introduced by Todd and Coxeter [133] enumerates the cosets of a subgroup in a finitely presented group. If the subgroup has finite index, cosetenumeration terminates and it computes a permutation representation for the group's action on the cosets. Coset-enumeration is a partial algorithm as the process will not 
terminate if the subgroup has infinite index. However, finite presentations often allow total algorithms that compute factor groups of special type (including abelian quotients, nilpotent quotients [107] and, in general, solvable quotients [93]).

Beside quotient and subgroup methods, the well-known theorem by Reidemeister [119] and Schreier 124 allows one to compute a presentation for a subgroup. The Reidemeister-Schreier Theorem explicitly shows that a finite index subgroup of a finitely presented group is itself finitely presented. A similar result can be shown for finite index ideals in finitely presented semi-groups [31. In practice, a permutation representation for the group's action on the cosets allows one to compute the Schreier generators of the subgroup and the Reidemeister rewriting. The Reidemeister rewriting can be used to rewrite the relations of the group to relations of the subgroup [72, 95, 129]. A method for computing a finite presentation for a finite index subgroup can be applied in the investigation of the structure of a group by its finite index subgroups [77].

Even though finitely presented groups have been studied for a long time, most groups are not finitely presented because there are uncountably many two-generator groups [103] but only countably many finite presentations [6]. A generalization of finite presentations are finite L-presentations which were introduced in [6]; however, there are still only countably many finite $L$-presentations. It is known that various examples of self-similar or branch groups (including the Grigorchuk group [53] and its twisted twin [14]) are finitely $L$-presented but not finitely presented [6]. Finite $L$-presentations are possibly infinite presentations with finitely many generators whose relations (up to finitely many exceptions) are obtained by iteratively applying finitely many substitutions to a finite set of relations; see [6] or Section D.2 for a definition. A finite $L$-presentation is invariant if the substitutions which generate the relations induce endomorphisms of the group. In fact, invariant finite $L$-presentations are finite presentations in the universe of groups with operators [87,111] in the sense that the operator domain of the group generates the possibly infinitely many relations out of a finite set of relations.

Finite $L$-presentations allow computer algorithms to be applied in the investigation of the groups they define. For instance, they allow to compute the lower central series quotients [9], the Dwyer quotients of the group's Schur multiplier [66], and even a cosetenumeration process exists for finitely $L$-presented groups [67]. It is the aim of this paper to prove the following variant of the well-known Reidemeister-Schreier Theorem:

Theorem 1.1 Each finite index subgroup of a finitely L-presented group is finitely Lpresented.

If the finite index subgroup in Theorem 1.1 is normal and invariant under the substitutions (i.e., a normal and admissible subgroup in the notion of Krull \& Noether [87,111]), an easy argument gives a finite $L$-presentation for the subgroup; furthermore, if the group is invariantly finitely $L$-presented, so is the subgroup. However, more work is needed if the subgroup is not invariant under the substitutions. Under either of two extra conditions (the subgroup is leaf-invariant, see Definition 5.20, or it is normal and weakly leaf-invariant, see Definition (7.36), we show that the subgroup is invariantly finitely $L$ presented as soon as the group is. We have not been able to get rid of these extra 
assumptions. In particular, it is not clear whether a finite index subgroup of an invariantly finitely $L$-presented group is always invariantly finitely $L$-presented. We show that the methods presented in this paper will (in general) fail to compute invariant $L$ presentations for the subgroup even if the group is invariantly $L$-presented. However, we are not aware of a method to prove that a given subgroup does not admit an invariant finite $L$-presentation at all.

Our proof of Theorem 1.1 is constructive and it yields a finite $L$-presentation for the subgroup. These finite $L$-presentations can be applied in the investigation of the underlying groups as the methods in [77] suggest for finitely presented groups. Notice that Theorem 1.1 was already posed in Proposition 2.9 of [6]. The proof we explain in this paper follows the sketch given in [6], but fixes a gap as the $L$-presentation of the group in Theorem 1.1 is possibly non-invariant. Even if the $L$-presentation is assumed to be invariant, the considered subgroup cannot be assumed to be invariant under the substitutions.

This paper is organized as follows: In Section D.2, we recall the notion of a finite $L$-presentation and we recall basic group theoretic constructions which preserve the property of being (invariantly) finitely $L$-presented. Then, in Section D.3. we recall the well-known Reidemeister-Schreier process. Before we prove Theorem 1.1 in Section D.6. we construct, in Section D.4, a counter-example to the original proof of Theorem 1.1 in [6]. Then, in Section D.5, we introduce the stabilizing subgroups which are the main tools in our proof of Theorem 1.1. In Section D.7, we study conditions on the finite index subgroup of an invariantly $L$-presented group to be invariantly $L$-presented itself. We conclude this paper by considering two examples of subgroup $L$-presentations in Section D.8 including the normal closure of a generator $d$ of the Grigorchuk group $\mathfrak{G}$ as in [11, 58. We fix a mistake in the generating set of the normal closure $D=\langle d\rangle^{\mathfrak{G}}$ using our Reidemeister-Schreier Theorem for finitely $L$-presented groups. In particular we show, in the style of [77], how these computational methods can be applied in the investigation of self-similar groups.

\section{D.2 Preliminaries}

In the following, we briefly recall the notion of a finite $L$-presentation and the notion a finitely $L$-presented group as introduced in [6]. Moreover, we recall some basic constructions for finite $L$-presentations.

A finite L-presentation is a group presentation of the form

$$
\left\langle\mathcal{X} \mid \mathcal{Q} \cup \bigcup_{\sigma \in \Phi^{*}} \mathcal{R}^{\sigma}\right\rangle,
$$

where $\mathcal{X}$ is a finite alphabet, $\mathcal{Q}$ and $\mathcal{R}$ are finite subsets of the free group $F$ over $\mathcal{X}$, and $\Phi^{*} \subseteq \operatorname{End}(F)$ denotes the free monoid of endomorphisms which is finitely generated by $\Phi$. We also write $\langle\mathcal{X}|\mathcal{Q}| \Phi \mid \mathcal{R}\rangle$ for the finite $L$-presentation in Eq. (D.1) and $G=\langle\mathcal{X}|\mathcal{Q}| \Phi \mid \mathcal{R}\rangle$ for the group it defines. 
A group which admits a finite $L$-presentation is finitely $L$-presented. An $L$-presentation of the form $\langle\mathcal{X}|\emptyset| \Phi \mid \mathcal{R}\rangle$ is ascending and an $L$-presentation $\langle\mathcal{X}|\mathcal{Q}| \Phi \mid \mathcal{R}\rangle$ is invariant (and the group it presents is invariantly L-presented), if each endomorphism $\varphi \in \Phi$ induces an endomorphism of the group; that is, if the normal subgroup $\left\langle\mathcal{Q} \cup \bigcup_{\sigma \in \Phi^{*}} \mathcal{R}^{\sigma}\right\rangle^{F} \leq F$ is $\varphi$-invariant. Each ascending $L$-presentation is invariant and each invariant $L$-presentation $\langle\mathcal{X}|\mathcal{Q}| \Phi \mid \mathcal{R}\rangle$ admits an ascending $L$-presentation $\langle\mathcal{X}|\emptyset| \Phi \mid \mathcal{Q} \cup \mathcal{R}\rangle$ which defines the same group. On the other hand, we have the following

Proposition 2.2 There are finite L-presentations that are not invariant.

Proof. The group $B=\left\langle\{a, b, t\} \mid\left\{a^{t} a^{-4}, b^{t^{-1}} b^{-2},\left[a, b^{t^{i}}\right] \mid i \in \mathbb{Z}\right\}\right\rangle$ is a met-abelian, infinitely related group with trivial Schur multiplier [18]. By introducing a stable letter $u$, this group admits the finite $L$-presentation

$$
\left\langle\{a, b, t, u\}\left|\left\{u b^{-1}\right\}\right|\{\sigma, \delta\} \mid\left\{a^{t} a^{-4}, b^{t^{-1}} b^{-2},[a, u]\right\}\right\rangle,
$$

where $\sigma$ is the free group homomorphism induced by the map $\sigma: a \mapsto a, b \mapsto b, t \mapsto t$, and $u \mapsto u^{t}$, while $\delta$ is the free group homomorphism induced by the map $\delta: a \mapsto a, b \mapsto b$, $t \mapsto t$, and $u \mapsto u^{t^{-1}}$. This finite $L$-presentation is not invariant [64.

Another non-invariant $L$-presentation can be given for the free product $\mathbb{Z}_{2} * \mathbb{Z}_{2}=\langle\{a, b\}|$ $\left.\left\{a^{2}, b^{2}\right\}\right\rangle$ : it is finitely $L$-presented by $\left\langle\{a, b\}\left|\left\{a^{2}\right\}\right|\{\sigma\} \mid\left\{b^{2}\right\}\right\rangle$ where $\sigma$ is induced by the map $a \mapsto a b$ and $b \mapsto b^{2}$. If this latter $L$-presentation were invariant, the ascending finite $L$-presentation $\left\langle\{a, b\}|\emptyset|\{\sigma\} \mid\left\{a^{2}, b^{2}\right\}\right\rangle$ would also define $\mathbb{Z}_{2} * \mathbb{Z}_{2}$. In this case $\left(a^{2}\right)^{\sigma}=a b a b$ is a relation and, since $a^{2}=b^{2}=1$ in the group, the generators $a$ and $b$ commute. Thus the latter ascending finite $L$-presentation defines a quotient of the 2-elementary abelian group $\mathbb{Z}_{2} \times \mathbb{Z}_{2}$. In particular, it defines a finite group. Hence, $\left\langle\{a, b\}|\emptyset|\{\sigma\} \mid\left\{a^{2}, b^{2}\right\}\right\rangle$ is not a finite $L$-presentation for $\mathbb{Z}_{2} * \mathbb{Z}_{2}$ and so $\left\langle\{a, b\}\left|\left\{a^{2}\right\}\right|\left\{\sigma^{2}\right\} \mid\left\{b^{2}\right\}\right\rangle$ is not an invariant $L$-presentation.

We are not aware of a method to decide whether or not a given (non-ascending) finite $L$-presentation is invariant. In particular, we have no answer to the following

Question 2.3 Is there a finitely L-presented group so that each of its finite L-presentation is not invariant?

The class of finitely $L$-presented groups contains all finitely presented groups:

Proposition 2.4 Each finitely presented group $\langle\mathcal{X} \mid \mathcal{R}\rangle$ is finitely L-presented by the invariant (ascending) finite L-presentation $\langle\mathcal{X}|\emptyset| \emptyset \mid \mathcal{R}\rangle$.

Therefore, (invariant or ascending) finite $L$-presentations generalize the concept of finite presentations. Examples of finitely $L$-presented, but not finitely presented, groups are various self-similar or branch groups [6] including the Grigorchuk group [53, 56, 96] and its twisted twin [14]. However, the concept of a finite $L$-presentation is quite general so that other examples of infinitely presented groups are finitely $L$-presented [18, 85. 
Various group theoretic constructions that preserve the property of being finitely $L$-presented have been studied in [6]. For completeness, we recall some of these constructions in the remainder of this section.

Proposition 2.5 (Bartholdi [6, Proposition 2.7]) Let $G=\langle\mathcal{X}|\mathcal{Q}| \Phi \mid \mathcal{R}\rangle$ be a finitely L-presented group and let $H=\langle\mathcal{Y} \mid \mathcal{S}\rangle$ be finitely presented. The group $K$ which satisfies the short exact sequence $1 \rightarrow G \rightarrow K \rightarrow H \rightarrow 1$ is finitely L-presented.

Proof. We recall the constructions from [6]: Let $\delta: H \rightarrow K$ be a section of $H$ to $K$ and identify $G$ with its image in $K$. Each relation $r \in \mathcal{S}$ of the finitely presented group $H$ lifts, through the section $\delta$, to an element $g_{r} \in G$. As the group $G$ is normal in $K$, each generator $t \in \mathcal{Y}$ of the finitely presented group $H$ acts, via $\delta$, on the subgroup $G$. Thus we have $x^{\sigma(t)}=g_{x, t} \in G$ for each $x \in \mathcal{X}$ and $t \in \mathcal{Y}$. If $\mathcal{X} \cap \mathcal{Y}=\emptyset$, we consider the finite $L$-presentation

$$
\left\langle\mathcal{X} \cup \mathcal{Y}\left|\mathcal{Q} \cup\left\{r g_{r}^{-1} \mid r \in \mathcal{S}\right\} \cup\left\{x^{t} g_{x, t}^{-1} \mid x \in \mathcal{X}, t \in \mathcal{Y}\right\}\right| \widehat{\Phi} \mid \mathcal{R}\right\rangle,
$$

where the endomorphisms $\Phi$ of $G$ 's $L$-presentation are extended to endomorphisms $\widehat{\Phi}=$ $\{\widehat{\sigma} \mid \sigma \in \Phi\}$ of the free group $F(\mathcal{X} \cup \mathcal{Y})$ by

$$
\widehat{\sigma}: F(\mathcal{X} \cup \mathcal{Y}) \rightarrow F(\mathcal{X} \cup \mathcal{Y}),\left\{\begin{array}{lll}
x & \mapsto x^{\sigma}, & \text { for each } x \in \mathcal{X} \\
y & \mapsto y, & \text { for each } y \in \mathcal{Y}
\end{array}\right.
$$

Then the finite $L$-presentation in Eq. (D.2) is a presentation for $K$; see [6].

As each finite group is finitely presented, Proposition 2.5 yields the immediate

Corollary 2.6 Each finite extension of a finitely L-presented group is finitely L-presented.

The construction in the proof of Proposition 2.5 gives a finite $L$-presentation for $K$ which is not ascending - even if the group $G$ we started with has an ascending $L$-presentation. We therefore ask the following

Question 2.7 Is every finite extension of an invariantly (finitely) L-presented group invariantly (finitely) L-presented?

We do not have an answer to this question in general; though we suspect its answer is negative, see Remark 7.43. Given endomorphisms $\Phi$ of the normal subgroup $G$ in Proposition 2.5, one problem is to construct endomorphisms of the finite extension $K$ which restrict to $\Phi$. This does not seem to be possible in general.

A finite $L$-presentation for a free product of two finitely $L$-presented groups is given by the following improved version of [6, Proposition 2.6].

Proposition 2.8 The free product of two finitely L-presented groups is finitely L-presented. If both finitely L-presented groups are invariantly L-presented, so is their free product. 
Proof. Although a proof of the first statement can be found in [6], we summarize its construction for our proof of the second statement. For this purpose, let $G=\langle\mathcal{X}|\mathcal{Q}| \Phi \mid \mathcal{R}\rangle$ and $H=\langle\mathcal{Y}|\mathcal{S}| \Psi \mid \mathcal{T}\rangle$ be finitely $L$-presented groups. Suppose that $\mathcal{X} \cap \mathcal{Y}=\emptyset$ holds. Then, by [6], the free product $G * H$ is finitely $L$-presented by $\langle\mathcal{X} \cup \mathcal{Y}|\mathcal{Q} \cup \mathcal{S}| \widehat{\Phi} \cup \widehat{\Psi}|$ $R \cup \mathcal{T}\rangle$ where the endomorphisms in $\Phi$ and $\Psi$ are extended to endomorphisms in $\widehat{\Phi}$ and $\widehat{\Psi}$ of the free group $F(\mathcal{X} \cup \mathcal{Y})$ over $\mathcal{X} \cup \mathcal{Y}$ as follows: for each $\sigma \in \Phi$, we let

$$
\widehat{\sigma}: F(\mathcal{X} \cup \mathcal{Y}) \rightarrow F(\mathcal{X} \cup \mathcal{Y}),\left\{\begin{aligned}
x & \mapsto x^{\sigma}, & \text { for each } x \in \mathcal{X} \\
y & \mapsto y, & \text { for each } y \in \mathcal{Y}
\end{aligned}\right.
$$

and, accordingly, for each $\delta \in \Psi$.

Suppose that the $L$-presentations of $G$ and $H$ are invariant. As an invariant $L$-presentation $\langle\mathcal{X}|\mathcal{Q}| \Phi \mid \mathcal{R}\rangle$ can be considered as an ascending $L$-presentation $\langle\mathcal{X}|\emptyset| \Phi|$ $\mathcal{Q} \cup \mathcal{R}\rangle$, we can consider $\mathcal{Q}$ and $\mathcal{S}$ to be empty. Then the construction above shows that the free product $G * H$ is ascendingly finitely $L$-presented and thus it is invariantly finitely $L$-presented.

We further have the following improved version of [6, Proposition 2.9]:

Proposition 2.9 Let $N \unlhd G$ be a normal subgroup of a finitely L-presented group $G=$ $\langle\mathcal{X}|\mathcal{Q}| \Phi \mid \mathcal{R}\rangle$. If $N$ is finitely generated as a normal subgroup, the factor group $G / N$ is finitely L-presented. If, furthermore, $G$ is invariantly L-presented and the normal subgroup $N$ is invariant under the induced endomorphisms $\Phi, G / N$ is invariantly Lpresented.

Proof. Let $N=\left\langle g_{1}, \ldots, g_{n}\right\rangle^{G}$ be a finite normal generating set of the normal subgroup $N$. We consider the normal generators $g_{1}, \ldots, g_{n}$ as elements of the free group $F$ over $\mathcal{X}$. By [6], the $L$-presentation $\left\langle\mathcal{X}\left|\mathcal{Q} \cup\left\{g_{1}, \ldots, g_{n}\right\}\right| \Phi \mid \mathcal{R}\right\rangle$ is a finite $L$-presentation for the factor group $G / N$.

Suppose that $G$ is given by an invariant $L$-presentation $\langle\mathcal{X}|\mathcal{Q}| \Phi \mid \mathcal{R}\rangle$. Then $G=\langle\mathcal{X}|\emptyset| \Phi \mid \mathcal{Q} \cup \mathcal{R}\rangle$. As $N^{\sigma} \subseteq N$, each $\sigma \in \Phi^{*}$ induces an endomorphism of the $L$-presented factor group $G / N$. Thus the images $g_{1}^{\sigma}, \ldots, g_{n}^{\sigma}$ are consequences of the relations of $G / N$ 's finite $L$-presentation above. Hence, we have that $G / N \cong\langle\mathcal{X}|$ $\left.\left\{g_{1}, \ldots, g_{n}\right\}|\Phi| \mathcal{R} \cup \mathcal{Q}\right\rangle=\left\langle\mathcal{X}|\emptyset| \Phi \mid \mathcal{Q} \cup \mathcal{R} \cup\left\{g_{1}, \ldots, g_{n}\right\}\right\rangle$.

If $G$ is invariantly $L$-presented and $N$ is a normal $\Phi$-invariant subgroup, then, in the notion of Krull \& Noether [87,111, the group $G$ is a group with operator domain $\Phi$ and the normal subgroup $N$ is an admissible subgroup. Proposition 2.8 and Proposition 2.9] yield the following straightforward

Corollary 2.10 Let $G$ and $H$ be finitely L-presented groups and let $F$ be a finitely generated group with embeddings $\psi: F \rightarrow G$ and $\phi: F \rightarrow H$. Then the amalgamated free product $G *_{F} H$ is finitely L-presented.

For further group theoretic constructions which preserve the property of being finitely $L$-presented were refer to [6]. 


\section{D.3 The Reidemeister-Schreier Process}

In the following, we briefly recall the Reidemeister-Schreier process as, for instance, outlined in [95, 129]. For this purpose, let $G$ be a group given by a group presentation $\langle\mathcal{X} \mid \mathcal{K}\rangle$ where $\mathcal{X}$ is a (finite) alphabet which defines the free group $F$ and $\mathcal{K} \subseteq F$ is a (possibly infinite) set of relations. Denote the normal closure of $\mathcal{K}$ in $F$ by $K=\langle\mathcal{K}\rangle^{F}$. Then $G=F / K$.

Let $H \leq G$ be a subgroup with finite index that is given by its generators $g_{1}, \ldots, g_{n}$ and let $\mathcal{T} \subseteq F$ be a Schreier transversal for $H$ in $G$ (i.e., a transversal for $H$ in $G$ so that every initial segment of an element of $\mathcal{T}$ itself belongs to $\mathcal{T}$, see [95]; note that we always act by multiplication from the right). We consider the generators of $H$ as words over the alphabet $\mathcal{X}$ and thus as elements of the free group $F$. Then the subgroup $U=\left\langle g_{1}, \ldots, g_{n}\right\rangle$ satisfies that $H \cong U K / K$. In the style of [95], we define the Schreier map $\gamma: \mathcal{T} \times \mathcal{X} \rightarrow F$ by $\gamma(t, x)=t x(\overline{t x})^{-1}$ where $\overline{t x}$ denotes the unique element $s \in \mathcal{T}$ from the Schreier transversal so that $U K s=U K t x$ holds. The Schreier Theorem (as, for instance, in [95, Proposition I.3.7]) shows that the subgroup $U K \leq F$ is freely generated by the Schreier generating set

$$
\mathcal{Y}=\{\gamma(t, x) \neq 1 \mid t \in \mathcal{T}, x \in \mathcal{X}\}
$$

In particular, it shows that each finite index subgroup of a finitely generated group is finitely generated. We consider the set $\mathcal{Y}$ as an alphabet and we denote by $F(\mathcal{Y})$ the free group over $\mathcal{Y}$. The Reidemeister rewriting $\tau$ is a map $\tau: F \rightarrow F(\mathcal{Y})$ given by

$$
\tau\left(y_{1} \cdots y_{n}\right)=\gamma\left(1, y_{1}\right) \cdot \gamma\left(\overline{y_{1}}, y_{2}\right) \cdots \gamma\left(\overline{y_{1} \cdots y_{n-1}}, y_{n}\right)
$$

where each $y_{i} \in \mathcal{X} \cup \mathcal{X}^{-}$. In general, the Reidemeister rewriting $\tau$ is not a group homomorphism. However, we have the following

Lemma 3.11 For $V \leq U K$, the restriction $\tau: V \rightarrow F(\mathcal{Y})$ is a homomorphism.

Proof. Let $g, h \in V$ be given. Write $g=g_{1} \cdots g_{n}$ and $h=h_{1} \cdots h_{m}$ with each $h_{i}, g_{j} \in$ $\mathcal{X} \cup \mathcal{X}^{-}$. Then, as $\overline{g_{1} \cdots g_{n}}=\bar{g}=1$ holds, we obtain that

$$
\tau(g h)=\gamma\left(1, g_{1}\right) \cdots \gamma\left(\overline{g_{1} \cdots g_{n-1}}, g_{n}\right) \cdot \gamma\left(1, h_{1}\right) \cdots \gamma\left(\overline{h_{1} \cdots h_{m-1}}, h_{m}\right)=\tau(g) \tau(h)
$$

while we already have $\tau(1)=1$ by definition.

By Schreier's theorem, the Reidemeister rewriting $\tau: U K \rightarrow F(\mathcal{Y})$ gives an isomorphism of free groups. A group presentation for the subgroup $H \cong U K / K$ is given by the following well-known theorem of Reidemeister [119] and Schreier [124]; see also [95, Section II.4].

Theorem 3.12 (Reidemeister-Schreier Theorem) Let $H$ be a subgroup of $G$. If $\tau$ denotes the Reidemeister-Schreier rewriting, $\mathcal{T}$ denotes a Schreier transversal for $H$ in $G$, and if $\langle\mathcal{X} \mid \mathcal{K}\rangle$ is a presentation for $G$, the subgroup $H$ is presented by

$$
H \cong\left\langle\mathcal{Y} \mid\left\{\tau\left(t r t^{-1}\right) \mid r \in \mathcal{K}, t \in \mathcal{T}\right\}\right\rangle .
$$


Proof. We recall the proof for completeness: Note that $H \cong U K / K \cong \tau(U K) / \tau(K)$ holds. By Schreier's theorem, we have $\tau(U K)=F(\mathcal{Y})$. It therefore suffices to determine a normal generating set for $\tau(K)$. As $\mathcal{K}$ is a normal generating set for $K \unlhd F$, a generating set for $\tau(K)$ is given by $\tau(K)=\left\langle\left\{\tau\left(g r g^{-1}\right) \mid r \in \mathcal{K}, g \in F\right\}\right\rangle$. Let $r \in \mathcal{K}$ and $g \in$ $F$ be given. Consider the relation $\tau\left(g r g^{-1}\right)$. Since $\mathcal{T}$ is a transversal for $U K$ in $F$, $g \in F$ can be written as $g=u t$ with $t \in \mathcal{T}$ and $u \in U K$. This yields $\tau\left(g r g^{-1}\right)=$ $\left.\tau(u \operatorname{urt})^{-1} u^{-1}\right)$. For $r \in \mathcal{K}$, we have that $\operatorname{trt}^{-1} \in U K$. By Lemma 3.11, we obtain that $\tau\left(g r g^{-1}\right)=\tau\left(u t r t^{-1} u^{-1}\right)=\tau(u) \tau\left(\operatorname{trt}^{-1}\right) \tau(u)^{-1}$. Therefore the relation $\tau\left(g_{r g}{ }^{-1}\right)$ is a consequence of $\tau\left(t r t^{-1}\right)$. Hence, it suffices to consider the normal generating set $\tau(K)=\left\langle\left\{\tau\left(t r t^{-1}\right) \mid r \in \mathcal{K}, t \in \mathcal{T}\right\}\right\rangle^{F(\mathcal{Y})}$ for $\tau(K)$.

If $H$ is a finite index subgroup of a finitely presented group $G$, there exist a finite set of relations $\mathcal{K}$ and a finite Schreier transversal $\mathcal{T}$ so that the subgroup $H$ is finitely presented by Theorem 3.12. This latter result for finitely presented groups is well-known and it is often simply referred to the Reidemeister-Schreier Theorem for finitely presented groups. In this paper, we prove a variant of the Reidemeister-Schreier Theorem for finitely $L$ presented groups using the ideas of Theorem 3.12 .

\section{D.4 A Typical Example of a Subgroup $L$-Presentation}

Before proving Theorem 1.1, we first consider an example of a finite $L$-presentation for a finite index subgroup of a finitely $L$-presented group. For this purpose we consider a subgroup of the Basilica group [60]. The Basilica group satisfies the following

Proposition 4.13 (Bartholdi \& Virág [16]) The Basilica group $G$ is invariantly finitely L-presented by $G \cong\left\langle\{a, b\}|\emptyset|\{\sigma\} \mid\left\{\left[a, a^{b}\right]\right\}\right\rangle$ where $\sigma$ is the free group homomorphism that is induced by the map $a \mapsto b^{2}$ and $b \mapsto a$.

The substitution $\sigma$ in Proposition 4.13 induces an endomorphism of $G$. The group $G$ will often provide an exclusive (counter-) example throughout this paper.

Consider the subgroup $H=\left\langle a, b a b^{-1}, b^{3}\right\rangle$ of the Basilica group. Then coset-enumeration for finitely $L$-presented groups 67] shows that $H$ is a normal subgroup of $G$ with index 3. A Schreier generating set for the subgroup $H$ is given by $\left\{a, b a b^{-1}, b^{2} a b^{-2}, b^{3}\right\}$. Write $x_{1}=a, x_{2}=b a b^{-1}, x_{3}=b^{2} a b^{-2}$, and $x_{4}=b^{3}$. Denote the free group over $\{a, b\}$ by $F$ and let $E$ denote the free group over $\left\{x_{1}, x_{2}, x_{3}, x_{4}\right\}$. For each $n \in \mathbb{N}_{0}$, we define $a_{n}=\left(2^{n}+2\right) / 3$ and $b_{n}=\left(2^{n}+1\right) / 3$. Then the $\sigma$-images of the relation $r=\left[a, a^{b}\right]$ can be rewritten with the Reidemeister rewriting $\tau: F \rightarrow E$. Their images have the form

$$
\tau\left(r^{\sigma^{2 n}}\right)= \begin{cases}{\left[x_{1}^{2^{n}}, x_{4}^{-a_{n}} x_{3}^{2^{n}} x_{4}^{a_{n}}\right],} & \text { if } n \text { is even, } \\ {\left[x_{1}^{2^{n}}, x_{4}^{-b_{n}} x_{2}^{2^{n}} x_{4}^{b_{n}}\right],} & \text { if } n \text { is odd, }\end{cases}
$$

and

$$
\tau\left(r^{\sigma^{2 n+1}}\right)=\left\{\begin{array}{cl}
x_{4}^{-b_{n+1}} x_{2}^{-2^{n}} x_{4}^{-b_{n+1}} x_{3}^{2^{n}} x_{4}^{b_{n+1}} x_{2}^{-2^{n}} x_{4}^{b_{n+1}} x_{1}^{2^{n}}, & \text { if } n \text { is even } \\
x_{4}^{-a_{n+1}} x_{3}^{-2^{n}} x_{4}^{-a_{n+1}+1} x_{2}^{2^{n}} x_{4}^{a_{n+1}-1} x_{3}^{-2^{n}} x_{4}^{a_{n+1}} x_{1}^{2^{n}}, & \text { if } n \text { is odd }
\end{array}\right.
$$


Note that $\tau\left(r^{\sigma^{2 n}}\right) \in[E, E]$ while $\tau\left(r^{\sigma^{2 n+1}}\right) \notin[E, E]$. Therefore, the images $\tau\left(r^{\sigma^{i}}\right)$ split into two classes which are recursive images of the endomorphism

$$
\widehat{\sigma}: E \rightarrow E, \begin{cases}x_{1} & \mapsto x_{1}^{2}, \\ x_{2} & \mapsto x_{3}^{2}, \\ x_{3} & \mapsto x_{4} x_{2}^{2} x_{4}^{-1}, \\ x_{4} & \mapsto x_{4}^{2}\end{cases}
$$

in the sense that $\widehat{\sigma}$ satisfies

$$
\tau\left(r^{\sigma^{2 n}}\right)=\left[x_{1}, x_{4}^{-1} x_{3} x_{4}\right]^{\widehat{\sigma}^{n}} \text { and } \tau\left(r^{\sigma^{2 n+1}}\right)=\left(x_{4}^{-1} x_{2}^{-1} x_{4}^{-1} x_{3} x_{4} x_{2}^{-1} x_{4} x_{1}\right)^{\widehat{\sigma}^{n}},
$$

for each $n \in \mathbb{N}_{0}$. In Section D.8, we show that a finite $L$-presentation for the subgroup $H$ is given by

$$
H \cong\left\langle\left\{x_{1}, \ldots, x_{4}\right\}|\emptyset|\{\widehat{\sigma}, \delta\} \mid\left\{\left[x_{1}, x_{4}^{-1} x_{3} x_{4}\right], x_{4}^{-1} x_{2}^{-1} x_{4}^{-1} x_{3} x_{4} x_{2}^{-1} x_{4} x_{1}\right\}\right\rangle
$$

where the endomorphism $\delta$ is induced by the map

$$
\delta: E \rightarrow E, \begin{cases}x_{1} & \mapsto x_{2}, \\ x_{2} & \mapsto x_{3}, \\ x_{3} & \mapsto x_{4} x_{1} x_{4}^{-1}, \\ x_{4} & \mapsto x_{4} .\end{cases}
$$

These subgroup $L$-presentations are typical for finite index subgroups of a finitely $L$ presented group. Besides, the subgroup $H$ and its subgroup $L$-presentation provide a counter-example to the original proof of Theorem 1.1 in [6] as there is no endomorphism $\varepsilon$ of the free group $E$ such that $\tau\left(r^{\sigma^{n+1}}\right)=\left(\tau\left(r^{\sigma^{n}}\right)\right)^{\varepsilon}$ holds for each $n \in \mathbb{N}_{0}$. A reason for the failure of the proof in [6] is that the subgroup $H$ is not $\sigma$-invariant but $\sigma^{2}$-invariant. Therefore, the method suggested in the proof of [6, Proposition 2.9] will fail to compute a finite $L$-presentation for $H$.

\section{D.5 Stabilizing Subgroups}

In this section, we introduce the stabilizing subgroups. These subgroups will be central to what follows.

Let $G=\langle\mathcal{X}|\mathcal{Q}| \Phi \mid \mathcal{R}\rangle$ be a finitely $L$-presented group and let $H \leq G$ be a finite index subgroup which is generated by $g_{1}, \ldots, g_{n}$. Denote the free group over $\mathcal{X}$ by $F$ and let $K=\left\langle\mathcal{Q} \cup \bigcup_{\sigma \in \Phi^{*}} \mathcal{R}^{\sigma}\right\rangle^{F}$. We consider the generators $g_{1}, \ldots, g_{n}$ of the subgroup $H$ as words over the alphabet $\mathcal{X} \cup \mathcal{X}^{-}$. Then the subgroup $U=\left\langle g_{1}, \ldots, g_{n}\right\rangle \leq F$ satisfies $H \cong U K / K$. The group $F$ acts on the right-cosets $U K \backslash F$ by multiplication from the right. Let $\pi: F \rightarrow \operatorname{Sym}(U K \backslash F)$ be a permutation representation for the group's action on $U K \backslash F$. Such a permutation representation can be computed with the coset-enumeration process from [67. The kernel of the permutation representation $\pi$ is the normal core, Core $_{F}(U K)$, of $U K$ in $F$; i.e., it is the largest normal subgroup of $F$ that is contained in $U K$.

The following definition introduces the stabilizing subgroups of $H$. These subgroups will be central to our proof of Theorem 1.1 in Section D.6. 
Definition 5.14 Let $G=\langle\mathcal{X}|\mathcal{Q}| \Phi \mid \mathcal{R}\rangle$ be a finitely L-presented group and let $H \leq G$ be a finite index subgroup which admits the permutation representation $\pi: F \rightarrow$ $\operatorname{Sym}(U K \backslash F)$ as above. The stabilizing subgroup of $H$ is

$$
\tilde{L}=\bigcap_{\sigma \in \Phi^{*}}(\sigma \pi)^{-1}\left(\operatorname{Stab}_{\operatorname{Sym}(U K \backslash F)}(U K 1)\right)=\bigcap_{\sigma \in \Phi^{*}} \sigma^{-1}(U K) .
$$

The stabilizing core of $H$ is

$$
L=\bigcap_{\sigma \in \Phi^{*}} \operatorname{ker}(\sigma \pi)
$$

For $\sigma \in \Phi^{*}$, we denote by $\|\sigma\|$ the usual word-length in the generating set $\Phi$ of the free monoid $\Phi^{*}$. The free monoid $\Phi^{*}$ has the structure of a $|\Phi|$-regular tree with its root being the identity map id: $F \rightarrow F$. We can further endow the monoid $\Phi^{*}$ with a lengthplus-(from the right)-lexicographic ordering $\prec$ by choosing an arbitrary ordering on the finite generating set $\Phi$. We then define $\sigma \prec \delta$ if $\|\sigma\|<\|\delta\|$ or, otherwise, if $\sigma=\sigma_{1} \cdots \sigma_{n}$ and $\delta=\delta_{1} \cdots \delta_{n}$, with each $\sigma_{i}, \delta_{j} \in \Phi$, and there exists a positive integer $1 \leq k \leq n$ such that $\sigma_{i}=\delta_{i}$ for each $k<i \leq n$, and $\sigma_{k} \prec \delta_{k}$. Since $\Phi$ is finite, the constructed ordering $\prec$ is a well-ordering on $\Phi^{*}[129$. Thus, there is no infinite descending sequences $\sigma_{1} \succ \sigma_{2} \succ \ldots$ in $\Phi^{*}$.

We consider a variation of the algorithm Is VALIDPERMREP from [67] in Algorithm D.1 below. If $\pi: F \rightarrow \operatorname{Sym}(U K \backslash F)$ denotes a permutation representation as in Defini-

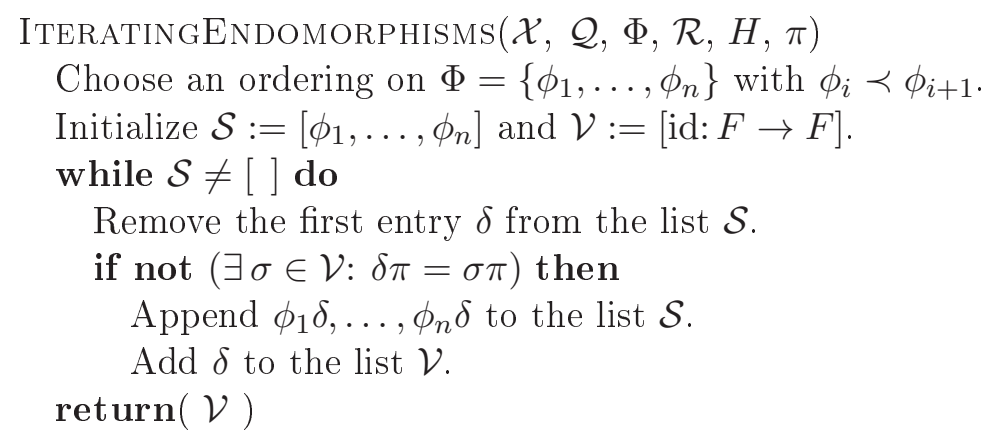

Algorithm D.1: Computing a finite set of endomorphisms $\mathcal{V} \subseteq \Phi^{*}$.

tion 5.14, the algorithm IteratingEndomorphisms returns a finite image of a section of the map $\Phi^{*} \rightarrow \operatorname{Hom}(F, \operatorname{Sym}(U K \backslash F))$ defined by $\sigma \mapsto \sigma \pi$; see Lemma 5.15 and Lemma 5.17 below. More precisely, we have the following

Lemma 5.15 The algorithm ITERATINGENDOMORPHISMS terminates and it returns a finite set of endomorphisms $\mathcal{V} \subseteq \Phi^{*}$ satisfying the following property: For each $\sigma \in \Phi^{*}$ there exists a unique $\tau \in \mathcal{V}$ so that $\sigma \pi=\tau \pi$. The element $\tau \in \mathcal{V}$ is minimal with respect to the ordering $\prec$ constructed above.

Proof. Let $\mathcal{X}$ be a basis of the free group $F$. A homomorphism $\psi: F \rightarrow \operatorname{Sym}(U K \backslash F)$ is uniquely defined by the image of this basis. Since $U K \backslash F$ is finite, the symmetric group 
$\operatorname{Sym}(U K \backslash F)$ is finite. Moreover, as $F$ is finitely generated, the set of homomorphisms $\operatorname{Hom}(F, \operatorname{Sym}(U K \backslash F))$ is finite. Therefore, the algorithm IteratingEndomorPhisms can add only finitely many elements to $\mathcal{V}$ and the stack $\mathcal{S}$ will eventually be reduced. Thus the algorithm terminates.

The ordering $\prec$ on $\Phi$ extends to a total well-ordering on the free monoid $\Phi^{*}$ as described above. The elements in the stack $\mathcal{S}$ are always ordered with respect to $\prec$. They further always succeed those elements in $\mathcal{V}$. In particular, the elements $\sigma \in \mathcal{V} \subseteq \Phi^{*}$ are $\prec$-minimal representatives of the composed homomorphism $\sigma \pi: F \rightarrow \operatorname{Sym}(U K \backslash F)$.

Let $\sigma \in \Phi^{*}$ be given and write $\sigma_{1}=\sigma$. There exists $w \in \Phi^{*}$ minimal subject to the existence of $\delta \in \mathcal{V}$ so that $\sigma_{1}=w \delta$. If $\|w\|=0$ holds, then $\sigma_{1} \in \mathcal{V}$ and the claim is proved. Otherwise, there exists $\psi \in \Phi$ so that $\sigma_{1}=v \psi \delta$ for some $v \in \Phi^{*}$ and $\psi \delta \notin \mathcal{V}$. Our algorithm yields the existence of $\varepsilon \in \mathcal{V}$ so that $\varepsilon \prec \psi \delta$ and $\psi \delta \pi=\varepsilon \pi$. We also have that $\sigma_{2}=v \varepsilon \prec v \psi \delta=\sigma_{1}$. This rewriting process yields a descending sequence $\sigma_{1} \succ \sigma_{2} \succ \ldots$ in $\Phi^{*}$. As $\prec$ is a well-ordering, there exists $\sigma_{n} \in \mathcal{V}$ so that $\sigma_{1} \succ \sigma_{2} \succ \ldots \succ \sigma_{n}$ and $\sigma \pi=\sigma_{1} \pi=\sigma_{n} \pi$. The element $\tau=\sigma_{n}$ is unique.

If $\pi: F \rightarrow \operatorname{Sym}(U K \backslash F)$ is a permutation representation for an infinite index subgroup $U K \leq F$, we cannot ensure finiteness of the set $\mathcal{V}$ and termination of the algorithm. In the remainder, we always consider finite index subgroups $U K \leq F$ only.

For finite $L$-presentations $\langle\mathcal{X}|\mathcal{Q}| \Phi \mid \mathcal{R}\rangle$ with $\Phi=\{\sigma\}$, finiteness of the set $\left\{\sigma^{\ell} \pi \mid \ell \in \mathbb{N}_{0}\right\} \subseteq \operatorname{Hom}(F, \operatorname{Sym}(U K \backslash F))$ yields the following

Corollary 5.16 If $\Phi=\{\sigma\}$, there exist integers $0 \leq i<j$ with $\sigma^{j} \pi=\sigma^{i} \pi$.

The set $\mathcal{V} \subseteq \Phi^{*}$ returned by Algorithm D.1 satisfies the following

Lemma 5.17 The set $\mathcal{V}$ can be considered as a subtree of $\Phi^{*}$. The image of the finite set $\mathcal{V}$ and the image of the monoid $\Phi^{*}$ in $\operatorname{Hom}(F, \operatorname{Sym}(U K \backslash F))$ coincide.

Proof. The identity mapping id: $F \rightarrow F$ is contained in $\mathcal{V}$ and it represents the root of $\mathcal{V}$ and $\Phi^{*}$. Let $\sigma \in \mathcal{V}$ be given. Then either $\sigma \in \Phi$ or there exists $\psi \in \Phi$ and $\delta \in \Phi^{*}$ so that $\sigma=\psi \delta$. In the first case, id: $F \rightarrow F$ is a unique parent of $\sigma \in \Phi$. Otherwise, if $\sigma=\psi \delta$, we need to show that $\delta \in \mathcal{V}$ holds. Our algorithm IteratingEndomorphisms only adds elements from the stack $\mathcal{S}$ to $\mathcal{V}$. At some stage of the algorithm we had $\sigma=\psi \delta \in \mathcal{S}$. The latter element is added to the stack $\mathcal{S}$ as a child of the element $\delta$ and thus $\delta \in \mathcal{V}$. The second statement follows immediately from Algorithm D.1 and Lemma 5.15.

We define a binary relation $\sim$ on the free monoid $\Phi^{*}$ by defining $\sigma \sim \delta$ if and only if the unique element $\sigma_{n} \in \Phi^{*}$ in Lemma 5.15 coincides for both $\sigma$ and $\delta$. Thus $\sigma \sim \delta$ if and only if $\sigma \pi=\delta \pi$. This definition yields the immediate

Lemma 5.18 The relation $\sigma \sim \delta$ is an equivalence relation. Each equivalence class is represented by a unique element in $\mathcal{V}$ which is minimal with respect to the total and well-ordering $\prec$.

Recall that $\pi: F \rightarrow \operatorname{Sym}(U K \backslash F)$ is a permutation representation for the group's action on the right-cosets $U K \backslash F$. If $\mathcal{T}$ is a transversal for $U K$ in $F, \sigma \sim \delta$ implies that $U K t \cdot g^{\sigma}=U K t \cdot g^{\delta}$ for each $t \in \mathcal{T}$ and $g \in F$. We therefore obtain the following 
Lemma 5.19 If $\sigma \in \Phi^{*}$ satisfies $\sigma \pi=\pi$, the subgroup $U K$ is $\sigma$-invariant. There are $\sigma$-invariant subgroups $U K$ that do not satisfy $\sigma \pi=\pi$.

Proof. The first statement holds in general for a group acting on a set: As $\sigma \pi=\pi$, we have $U K t \cdot g^{\sigma}=U K t g$ for each $t \in \mathcal{T}$ and $g \in F$. If $g \in U K$, then $U K 1 \cdot g^{\sigma}=U K 1 \cdot g=$ $U K 1$ and so $g^{\sigma} \in U K$. The index-2 subgroup $H=\left\langle a, b^{2}, b a b^{-1}\right\rangle$ of the Basilica group satisfies $(U K)^{\sigma} \subseteq U K$ and $\sigma \pi \neq \pi$. This (and similar results in the remainder of this paper) can be easily verified with a computer algebra system such as GAP.

The latter observation motivates the following

Definition 5.20 Let $G=\langle\mathcal{X}|\mathcal{Q}| \Phi \mid \mathcal{R}\rangle$ be a finitely L-presented group and let $H \leq G$ be a finite index subgroup with permutation representation $\pi$ as above. The $\pi$-leafs $\Psi \subseteq \Phi^{*} \backslash \mathcal{V}$ of $\mathcal{V}$ are

$$
\Psi=\{\psi \delta \mid \psi \in \Phi, \delta \in \mathcal{V}, \psi \delta \notin \mathcal{V}, \psi \delta \pi=\pi\} .
$$

The subgroup $H$ is leaf-invariant if $\Psi=\{\psi \delta \mid \psi \in \Phi, \delta \in \mathcal{V}, \psi \delta \notin \mathcal{V}\}$ holds.

For a finitely $L$-presented group $\langle\mathcal{X}|\mathcal{Q}| \Phi \mid \mathcal{R}\rangle$, the generating set $\Phi$ of $\Phi^{*}$ is finite. Moreover, the equivalence $\sim$ yields finitely many equivalence classes. Hence, the set of $\pi$-leafs $\Psi$ of $\mathcal{V}$ is finite. We obtain the following

Lemma 5.21 If $H$ is a leaf-invariant subgroup of $G$, each $\pi$-leaf $\psi \delta \in \Psi$ induces an endomorphism of UK. Moreover, each $\sigma \in \Phi^{*}$ can be written as $\sigma=v \sigma$ with $v \in \mathcal{V}$ and $\sigma \in \Psi^{*}$.

Proof. We again follow the ideas of Algorithm D.1. By Lemma 5.19, the condition $\psi \sigma \pi=\pi$ implies $\psi \sigma$-invariance of $U K$ and hence $\Psi^{*} \subseteq \operatorname{End}(U K)$. Write $W=\{\psi \delta \mid \psi \in$ $\Phi, \delta \in \mathcal{V}, \psi \delta \notin \mathcal{V}\}$ and let $\sigma \in \Phi^{*}$ be given. Write $\sigma_{1}=\sigma$. There exists $w \in \Phi^{*}$ minimal subject to the existence of $\delta \in \mathcal{V}$ so that $\sigma_{1}=w \delta$. If $\|w\|=0$, then $\sigma_{1}=\delta$ id with $\delta \in \mathcal{V}$ and id $\in \Psi^{*}$. Otherwise, there exists $\psi \in \Phi$ and $\sigma_{2} \in \Phi^{*}$ so that $\sigma_{1}=\sigma_{2} \psi \delta$ and $\psi \delta \notin \mathcal{V}$. Then $\psi \delta \in W$. Since $H$ is leaf-invariant, we have $W=\Psi$ and hence $\psi \delta \in \Psi$. Therefore $\psi \delta$ induces an endomorphism of $U K$. Clearly $\sigma_{2} \prec \sigma_{1}$. Rewriting the prefix $\sigma_{2}$ as above yields a descending sequence $\sigma_{1} \succ \sigma_{2} \ldots$ in $\Phi^{*}$. As $\prec$ is a well-ordering, we eventually have $\sigma_{1} \succ \sigma_{2} \succ \ldots \succ \sigma_{n}$ with $\sigma_{n} \in \mathcal{V}$ and $\sigma=\sigma_{1}=\sigma_{n} \delta$ for some $\delta \in \Psi^{*}$.

If the finite $L$-presentation $\langle\mathcal{X}|\mathcal{Q}| \Phi \mid \mathcal{R}\rangle$ satisfies $\Phi=\{\sigma\}$ and if there exists a minimal positive integer $0<j$ so that $\sigma^{j} \pi=\pi$, the set

$$
W=\{\psi \delta \mid \psi \in \Phi, \delta \in \mathcal{V}, \psi \delta \notin \mathcal{V}\}
$$

in the proof of Lemma 5.21 above becomes $W=\left\{\sigma^{j}\right\}$. Note the following

Remark 5.22 The condition $\sigma^{j} \pi=\sigma^{0} \pi$ is essential for the $\sigma^{j-0}$-invariance of the subgroup. For instance, the subgroup $H=\left\langle a, b a b^{-1}, b^{-1} a^{2} b, b^{4}, b^{2} a b^{-2}\right\rangle$ of the Basilica group satisfies $\sigma^{4} \pi=\sigma^{3} \pi$ but it is not $\sigma$-invariant.

The stabilizing subgroup $\tilde{L}$ introduced in Definition 5.14 satisfies the following 
Proposition 5.23 Let $\mathcal{V} \subseteq \Phi^{*}$ be the finite set returned by Algorithm D.1. The stabilizing subgroup $\tilde{L}$ satisfies that

$$
\tilde{L}=\bigcap_{\sigma \in \mathcal{V}}(\sigma \pi)^{-1}\left(\operatorname{Stab}_{\operatorname{Sym}(U K \backslash F)}(U K 1)\right)=\bigcap_{\sigma \in \mathcal{V}} \sigma^{-1}(U K) .
$$

The stabilizing subgroup $\tilde{L}$ is $\Phi$-invariant (i.e., we have $\tilde{L}^{\psi} \subseteq \tilde{L}$ for each $\psi \in \Phi$ ). It is contained in the subgroup $U K$ and it has finite index in $F$. The stabilizing subgroup $\tilde{L}$ is the largest $\Phi^{*}$-invariant subgroup of $U K$. It is not necessarily normal in $F$.

Proof. By Lemma 5.17, the sets $\left\{\sigma \pi \mid \sigma \in \Phi^{*}\right\}$ and $\{\sigma \pi \mid \sigma \in \mathcal{V}\}$ coincide and thus we have

$$
\tilde{L}=\bigcap_{\sigma \in \Phi^{*}}(\sigma \pi)^{-1}\left(\operatorname{Stab}_{\operatorname{Sym}(U K \backslash F)}(U K 1)\right)=\bigcap_{\sigma \in \mathcal{V}}(\sigma \pi)^{-1}\left(\operatorname{Stab}_{\operatorname{Sym}(U K \backslash F)}(U K 1)\right) .
$$

Since $(\sigma \pi)^{-1}\left(\operatorname{Stab}_{\operatorname{Sym}(U K \backslash F)}(U K 1)\right)=\sigma^{-1}(U K)$, we have $\tilde{L}=\bigcap_{\sigma \in \mathcal{V}} \sigma^{-1}(U K)$. For $\psi \in$ $\Phi$, we have

$$
\psi^{-1}(\tilde{L})=\bigcap_{\sigma \in \Phi^{*}}(\sigma \psi)^{-1}(U K) \supseteq \bigcap_{\sigma \in \Phi^{*}} \sigma^{-1}(U K)=\tilde{L}
$$

since the first intersection is over a smaller set than the second one. Thus $\psi(\tilde{L}) \subseteq \tilde{L}$. Since $\sigma=$ id $\in \Phi^{*}$, we have $\tilde{L} \subseteq U K$. Because the stabilizing subgroup $\tilde{L}$ is the intersection of finitely many finite index subgroups $(\sigma \pi)^{-1}\left(\operatorname{Stab}_{\operatorname{Sym}(U K \backslash F)}(U K 1)\right.$ of $F$, it has finite index in $F$. If $N \leq U K$ is $\Phi^{*}$ invariant, we have $N \subseteq \sigma^{-1}(N) \subseteq \sigma^{-1}(U K)$ for each $\sigma \in \Phi^{*}$. Hence $N \subseteq \bigcap_{\sigma \in \Phi^{*}} \sigma^{-1}(U K)=\tilde{L}$.

The stabilizing subgroup $\tilde{L}=\left\langle a, b a b^{-1}, b^{-1} a^{2} b, b^{2} a b^{-2}, b^{3} a^{-1} b, b^{-1} a b^{3}\right\rangle$ of the subgroup $H=\left\langle a, b a b^{-1}, b^{-1} a^{-2} b, b^{2} a b^{-2}, b^{3} a^{-1} b, b^{-1} a b^{3}\right\rangle$ of the Basilica group is not normal in $F$.

The stabilizing subgroup $\tilde{L}$ always satisfies that $\tilde{L} \subseteq U K$. Conditions for equality are given by the following

Lemma 5.24 The following conditions are equivalent:

(i) $\tilde{L}=U K$,

(ii) $(U K)^{\psi} \subseteq U K$ for all $\psi \in \mathcal{V}$, and

(iii) $(U K)^{\delta} \subseteq U K$ for all $\delta \in \Phi^{*}$.

Proof. We have that $\tilde{L}=\bigcap_{\sigma \in \Phi^{*}} \sigma^{-1}(U K)=\bigcap_{\sigma \in \mathcal{V}} \sigma^{-1}(U K)$. Therefore $\tilde{L}=\bigcap_{\sigma \in \Phi^{*}} \sigma^{-1}(U K)=U K$ if and only if $U K \subseteq \tilde{L} \subseteq \sigma^{-1}(U K)$ and so $(U K)^{\sigma} \subseteq U K$ for all $\sigma \in \Phi^{*}$. Similarly, we have $(U K)^{\psi} \subseteq U K$, for all $\psi \in \mathcal{V}$, if and only if $(U K)^{\sigma} \subseteq U K$, for all $\sigma \in \Phi^{*}$.

In the style of [67], we define a binary relation $\leadsto \pi$ on the free monoid $\Phi^{*}$ as follows: For $\sigma, \delta \in \Phi^{*}$ we define $\sigma \leadsto \pi \delta$ if and only if there exists a homomorphism $\gamma: \operatorname{im}(\delta \pi) \rightarrow$ $\operatorname{im}(\sigma \pi)$ so that $\sigma \pi=\delta \pi \gamma$ holds. It is known [67] that it is decidable whether or not $\sigma \sim_{\pi} \delta$ holds. This yields that 
Lemma 5.25 Let $\mathcal{V} \subseteq \Phi^{*}$ be the finite set returned by Algorithm D.1. Then there exists a subset $\tilde{\mathcal{V}} \subseteq \mathcal{V}$ with the following property: For each $\sigma \in \Phi^{*}$ there exists a unique element $\delta \in \tilde{\mathcal{V}}$ so that $\sigma \sim_{\pi} \delta$ and $\delta$ is minimal with respect to the ordering $\prec$ in Lemma 5.15.

Proof. This is straightforward as the set $\mathcal{V}$ returned by Algorithm D.1 is an upper bound on $\tilde{\mathcal{V}}$ because $\sigma \sim \delta$ implies both $\sigma \leadsto_{\pi} \delta$ or $\delta \sim_{\pi} \sigma$.

Again, the set $\tilde{\mathcal{V}}$ in Lemma 5.25 can be considered a subtree of $\Phi^{*}$ or even as a subtree of $\mathcal{V}$. The binary relation $\sim_{\pi}$ is reflexive and transitive but not necessarily symmetric. The equivalence relation $\sim$ and the relation $\sim \pi$ are related by the following

Lemma 5.26 Let $\pi: F \rightarrow \operatorname{Sym}(U K \backslash F)$ be a permutation representation as above. For $\sigma, \delta \in \Phi^{*}$, we have the following

(i) We have $\sigma \sim_{\pi} \delta$ and $\delta \hookrightarrow_{\pi} \sigma$ if and only if the homomorphism $\gamma: \operatorname{im}(\delta \pi) \rightarrow \operatorname{im}(\sigma \pi)$ with $\sigma \pi=\delta \pi \gamma$ is bijective.

(ii) If $\sigma \sim \delta$, then $\sigma \sim_{\pi} \delta$ and $\delta \hookrightarrow_{\pi} \sigma$. The converse is not necessarily true.

(iii) If $k>0$ is minimal so that $\sigma^{k} \sim \mathrm{id}$, there exists a minimal positive integer $\ell$ so that $\ell \mid k$ and $\sigma^{\ell} \hookrightarrow_{\pi}$ id. If $\Phi=\{\sigma\}$, the set $\tilde{\mathcal{V}}$ from Lemma 5.25 becomes $\tilde{\mathcal{V}}=\left\{\mathrm{id}, \sigma, \ldots, \sigma^{\ell-1}\right\}$.

(iv) If $\ell$ is a minimal positive integer such that id $\sim_{\pi} \sigma^{\ell}$, there exists a minimal integer $k \geq \ell$ so that $\sigma^{k} \sim \mathrm{id}$. If $\Phi=\{\sigma\}$, the set $\mathcal{V}$ returned by Algorithm D.1 becomes $\mathcal{V}=\left\{\mathrm{id}, \sigma, \ldots, \sigma^{k-1}\right\}$ while $\tilde{\mathcal{V}}=\left\{\mathrm{id}, \sigma, \ldots, \sigma^{\ell-1}\right\}$.

(v) The subgroup $H=\left\langle a, b^{2}, b a b^{-1}\right\rangle$ of the Basilica group satisfies $\sigma \sim_{\pi}$ id but there is no positive integer $\ell>0$ so that $\sigma^{\ell} \sim$ id holds.

Proof. If the homomorphism $\gamma: \operatorname{im}(\delta \pi) \rightarrow \operatorname{im}(\sigma \pi)$ with $\sigma \pi=\delta \pi \gamma$ is bijective, we obtain $\sigma \pi \gamma^{-1}=\delta \pi$ and thus $\delta \leadsto_{\pi} \sigma$. On the other hand, suppose that both $\delta \leadsto_{\pi} \sigma$ and $\sigma \leadsto_{\pi} \delta$ hold. Then there are homomorphisms $\gamma: \operatorname{im}(\sigma \pi) \rightarrow \operatorname{im}(\delta \pi)$ and $\tau: \operatorname{im}(\delta \pi) \rightarrow \operatorname{im}(\sigma \pi)$ so that $\delta \pi=\sigma \pi \gamma$ and $\sigma \pi=\delta \pi \tau$. This yields $\delta \pi=\sigma \pi \gamma=\delta \pi \tau \gamma$ and $\sigma \pi=\delta \pi \tau=\sigma \pi \gamma \tau$. Hence $\gamma$ and $\tau$ are isomorphisms.

Since $\sigma \sim \delta$ implies $\sigma \pi=\delta \pi$, we immediately obtain both $\sigma \sim_{\pi} \delta$ and $\delta \sim_{\pi} \sigma$. The subgroup $H=\left\langle a, b a b^{-1}, b^{3}\right\rangle$ of the Basilica group admits the permutation representation $\pi: a \mapsto(), b \mapsto(1,2,3)$. We have $\sigma^{2} \pi: a \mapsto(), b \mapsto(1,3,2)$ and therefore $\sigma^{2} \leadsto_{\pi}$ id and id $\sim_{\pi} \sigma^{2}$. Though $\sigma^{2} \pi \neq \pi$.

Suppose that $k \in \mathbb{N}$ is minimal so that $\sigma^{k} \sim$ id and so $\sigma^{k} \pi=\pi$. Then $\operatorname{im}(\pi) \supseteq$ $\operatorname{im}(\sigma \pi) \supseteq \ldots \supseteq \operatorname{im}\left(\sigma^{k} \pi\right)=\operatorname{im}(\pi)$. There exists a minimal integer $0<\ell \leq k$ such that $\sigma^{\ell} \sim_{\pi}$ id. Hence, there exists a homomorphism $\gamma: \operatorname{im}(\pi) \rightarrow \operatorname{im}\left(\sigma^{\ell} \pi\right)$ with $\sigma^{\ell} \pi=\pi \gamma$. The homomorphism $\gamma$ is onto and, since $\operatorname{im}(\pi)=\operatorname{im}\left(\sigma^{\ell} \pi\right)$ is finite, $\gamma$ is bijective. As $\ell \leq k$ holds, we can write $k=s \ell+t$ for some $0 \leq t<\ell$ and $s \in \mathbb{N}$. This yields that $\pi=\sigma^{k} \pi=\sigma^{t} \sigma^{s \ell} \pi=\sigma^{t} \pi \gamma^{s}$ and so $\pi \gamma^{-s}=\sigma^{t} \pi$. If $t>0$, the latter yields that $\sigma^{t} \sim_{\pi}$ id which contradicts the minimality of $\ell$. Thus $t=0$ and $\ell \mid k$. If $\Phi=\{\sigma\}$, the 
set $\left\{\mathrm{id}, \sigma, \ldots, \sigma^{\ell-1}\right\}$ is an upper bound on the set $\tilde{\mathcal{V}}$ from Lemma 5.25 because $\sigma^{\ell} \sim_{\pi}$ id holds. By the minimal choice of $\ell$, we obtain that $\tilde{\mathcal{V}}=\left\{\mathrm{id}, \sigma, \ldots, \sigma^{\ell-1}\right\}$.

Suppose that id $\sim_{\pi} \sigma^{\ell}$ holds. There exists a homomorphism $\gamma: \operatorname{im}\left(\sigma^{\ell} \pi\right) \rightarrow \operatorname{im}(\pi)$ with $\sigma^{\ell} \pi \gamma=\pi$. Since $\gamma$ is a surjective map from a subgroup $\operatorname{im}\left(\sigma^{\ell} \pi\right) \leq \operatorname{im}(\pi)$ onto $\operatorname{im}(\pi), \gamma$ is bijective and hence, we also have that $\sigma^{\ell} \sim_{\pi}$ id. Suppose that the automorphism $\gamma$ of the finite group $\operatorname{im}(\pi)$, has finite order $n$. Write $k=n \ell$. Then $\sigma^{k} \pi=\sigma^{n \ell} \pi=\pi \gamma^{n}=\pi$ and so $\sigma^{k} \sim$ id and $k$ is minimal. If $\Phi=\{\sigma\}$ holds, then, by the minimal choice of $k$, we obtain $\mathcal{V}=\left\{\right.$ id, $\left.\sigma, \ldots, \sigma^{k-1}\right\}$ for the set $\mathcal{V}$ returned by Algorithm D.1 while $\mathcal{V}=\left\{\right.$ id $\left., \sigma, \ldots, \sigma^{\ell-1}\right\}$ by the minimality of $\ell$.

The permutation representation $\pi: F \rightarrow \operatorname{Sym}(U K \backslash F)$ of the subgroup $H=$ $\left\langle a, b^{2}, b a b^{-1}\right\rangle$ is induced by the map $a \mapsto()$ and $b \mapsto(1,2)$. Therefore, $H$ satisfies that $\sigma \leadsto_{\pi} \operatorname{id},|\operatorname{im}(\pi)|=2$, and $|\operatorname{im}(\sigma \pi)|=1$. In particular, for each $\ell \geq 1$, we have $\left|\operatorname{im}\left(\sigma^{\ell} \pi\right)\right|=1$. Thus there is no integer $\ell$ so that $\sigma^{\ell} \sim$ id holds. However, we have $\sigma^{2} \pi=\sigma \pi$ so that the set $\mathcal{V}=\left\{\mathrm{id}, \sigma, \sigma^{2}\right\}$ returned by Algorithm D.1 is still finite.

The stabilizing core $L$ introduced in Definition 5.14 satisfies the following

Proposition 5.27 Let $\mathcal{V} \subseteq \Phi^{*}$ be the finite set returned by Algorithm D.1. The stabilizing core L satisfies that

$$
L=\bigcap_{\sigma \in \mathcal{V}} \operatorname{ker}(\sigma \pi) .
$$

The stabilizing core $L$ is the largest $\Phi$-invariant subgroup of $U K$ which is normal in $F$ and thus $L=\operatorname{Core}_{F}(\tilde{L})$. It is finitely generated, has finite index in $F$, and it contains all iterated relations $\mathcal{R}$ of $G$ 's L-presentation $\langle\mathcal{X}|\mathcal{Q}| \Phi \mid \mathcal{R}\rangle$. We have $L \subseteq \tilde{L} \subseteq U K \subseteq F$ and $L \subseteq \operatorname{Core}_{F}(U K) \subseteq U K \subseteq F$.

Proof. By Lemma 5.17, the sets $\left\{\sigma \pi \mid \sigma \in \Phi^{*}\right\}$ and $\{\sigma \pi \mid \sigma \in \mathcal{V}\}$ coincide and thus we have

$$
L=\bigcap_{\sigma \in \Phi^{*}} \operatorname{ker}(\sigma \pi)=\bigcap_{\sigma \in \mathcal{V}} \operatorname{ker}(\sigma \pi) .
$$

The stabilizing core $L$ is normal in $F$ because it is the intersection of normal subgroups. Since $L \subseteq \operatorname{ker}(\pi)=\operatorname{Core}_{F}(U K)$ holds, the stabilizing core $L$ is contained in $U K$. Since $\sigma^{-1}(\operatorname{ker}(\pi))=\operatorname{ker}(\sigma \pi)$, we have that $L=\bigcap_{\sigma \in \Phi^{*}} \sigma^{-1}(\operatorname{ker}(\pi))$. For any $\psi \in \Phi^{*}$, we obtain

$$
\psi^{-1}(L)=\bigcap_{\sigma \in \Phi^{*}}(\sigma \psi)^{-1}(\operatorname{ker}(\pi)) \supseteq \bigcap_{\sigma \in \Phi^{*}} \sigma^{-1}(\operatorname{ker}(\pi))=L
$$

as the first intersection is over a small set of indices. Thus $L$ is $\Phi^{*}$-invariant. Let $N \leq U K$ be a $\Phi^{*}$-invariant subgroup which is normal in $F$. Then $N \leq \operatorname{Core}_{F}(U K)=\operatorname{ker}(\pi)$ and so $N \subseteq \sigma^{-1}(N) \subseteq \sigma^{-1}(\operatorname{ker}(\pi))$ for each $\sigma \in \Phi^{*}$. Thus $N \subseteq \bigcap_{\sigma \in \Phi^{*}} \sigma^{-1}(\operatorname{ker}(\pi))=L$. The stabilizing core $L$ has finite index in $F$ because it is the intersection of finitely many finite index subgroups $\operatorname{ker}(\sigma \pi)$ with $\sigma \in \mathcal{V}$.

The stabilizing core $L$ is finitely generated as a finite index subgroup of a finitely generated free group $F$. Let $r \in \mathcal{R}$ be an iterated relator of $G$ 's $L$-presentation $\langle\mathcal{X}|\mathcal{Q}|$ 
$\Phi|\mathcal{R}\rangle$. Then, for each $\sigma \in \mathcal{V}$, the image $r^{\sigma}$ is a relator of $G$. Thus $r \in \operatorname{ker}(\sigma \pi)$ and $r \in L$.

As $L$ is a $\Phi$-invariant subgroup of $U K$, we have $L \subseteq \tilde{L}$ by Proposition 5.23. Moreover, $L \subseteq \operatorname{ker}(\pi)=\operatorname{Core}_{F}(U K)$.

Since the stabilizing core $L$ contains the iterated relations $\mathcal{R}$ of the $L$-presentation, it also contains the normal closure $\left\langle\bigcap_{\sigma \in \Phi^{*}} \mathcal{R}^{\sigma}\right\rangle^{F}$. We obtain the immediate

Corollary 5.28 If $G=\langle\mathcal{X}|\mathcal{Q}| \Phi \mid \mathcal{R}\rangle=\langle\mathcal{X}|\emptyset| \Phi \mid \mathcal{Q} \cup \mathcal{R}\rangle$ is invariantly L-presented, we have $K \subseteq L \subseteq \tilde{L} \subseteq U K \subseteq F$. The subgroup $H \cong U K / K \leq F / K$ contains the $\Phi$-invariant normal subgroup $L / K$. The index $[U K / K: L / K]=[U K: L]$ is finite.

The subgroup $H$ in Corollary 5.28 is a finite extension of $L / K$. Since the stabilizing core $L$ is the largest $\Phi$-invariant subgroup which is normal in $F$, the stabilizing subgroup $\tilde{L}$ is normal in $F$ if and only if $L=\tilde{L}$ holds. More precisely, we have the following

Lemma 5.29 We have $\tilde{L}=L$ if and only if $\tilde{L} \subseteq \operatorname{Core}_{F}(U K)$.

Proof. We have $L \subseteq \tilde{L}$ and $\tilde{L}^{\sigma} \subseteq \tilde{L}$ for each $\sigma \in \Phi^{*}$. If $L=\tilde{L}$, then $\tilde{L}=L \subseteq$ $\operatorname{Core}_{F}(U K)$. If $\tilde{L} \subseteq \operatorname{Core}_{F}(U K)=\operatorname{ker}(\pi)$, then $\tilde{L} \subseteq \sigma^{-1}(\tilde{L}) \subseteq \sigma^{-1}(\operatorname{ker}(\pi))$ for all $\sigma \in \Phi^{*}$. Thus $\tilde{L} \subseteq \bigcap_{\sigma \in \Phi^{*}} \sigma^{-1}(\operatorname{ker}(\pi))=L$.

If $U K \unlhd F$ is a normal subgroup, then $\tilde{L} \subseteq U K=\operatorname{Core}_{F}(U K)$. Hence, we obtain the immediate

Corollary 5.30 If $U K \unlhd F$, then $L=\tilde{L}$.

Note the following

Remark 5.31 There are subgroups that satisfy $\operatorname{Core}_{F}(U K) \subset \tilde{L}$. For instance, the subgroup $H=\left\langle a, b^{2}, b a^{2} b^{-1}, b a b^{-2} a^{-1} b^{-1}\right\rangle$ of the Basilica group is $\Phi$-invariant (and hence $\tilde{L}=U K$ by Lemma 5.24) but not normal in $G$.

There are subgroups that satisfy $\tilde{L} \subset \mathrm{Core}_{F}(U K)$. For instance, the subgroup $H=$ $\left\langle a^{2}, b, a b a^{-1}\right\rangle$ of the Basilica group has index 2 in $G$ (and thus it is normal in $G$ ); though the subgroup $H$ is not $\sigma$-invariant.

There are subgroups that neither satisfy $\tilde{L} \subseteq \operatorname{Core}_{F}(U K)$ nor $\operatorname{Core}_{F}(U K) \subseteq \tilde{L}$. For instance, the subgroup $H=\left\langle a, b a b^{-1}, b^{-1} a^{2} b, b^{2} a b^{2}, b^{3} a^{-1} b\right\rangle$ of the Basilica group satisfies $[F: \tilde{L}]=\left[F: \operatorname{Core}_{F}(U K)\right]$ and $\tilde{L} \neq \operatorname{Core}_{F}(U K)$.

\section{D.6 The Reidemeister-Schreier Theorem}

In this section, we finally prove our variant of the Reidemeister-Schreier Theorem in Theorem 1.1. For this purpose, let $G=\langle\mathcal{X}|\mathcal{Q}| \Phi \mid \mathcal{R}\rangle$ be a finitely $L$-presented group and let $H \leq G$ be a finite index subgroup given by its generators $g_{1}, \ldots, g_{n}$. We consider the generators $g_{1}, \ldots, g_{n}$ as elements of the free group $F$ over $\mathcal{X}$. Denote the normal closure of the relations of $G$ by $K=\left\langle\mathcal{Q} \cup \bigcup_{\sigma \in \Phi^{*}} \mathcal{R}^{\sigma}\right\rangle^{F}$ and let $U=\left\langle g_{1}, \ldots, g_{n}\right\rangle \leq$ 
$F$. Then $H \cong U K / K$. If $\mathcal{T} \subseteq F$ denotes a Schreier transversal for $U K$ in $F$, the Reidemeister-Schreier Theorem in Section D.3 shows that the subgroup $H$ admits the group presentation

$$
H \cong\left\langle\mathcal{Y} \mid\left\{\tau\left(t q t^{-1}\right) \mid t \in \mathcal{T}, q \in \mathcal{Q}\right\} \cup \bigcup_{\sigma \in \Phi^{*}}\left\{\tau\left(t r^{\sigma} t^{-1}\right) \mid t \in \mathcal{T}, r \in \mathcal{R}\right\}\right\rangle,
$$

where $\tau$ denotes the Reidemeister rewriting. We will construct a finite $L$-presentation from the group presentation in Eq. (D.7). First, we note the following

Theorem 6.32 Let $G=\langle\mathcal{X}|\mathcal{Q}| \Phi \mid \mathcal{R}\rangle$ be invariantly finitely L-presented. Each $\Phi$-invariant normal subgroup with finite index in $G$ is invariantly finitely L-presented.

Proof. Let $G=\langle\mathcal{X}|\mathcal{Q}| \Phi \mid \mathcal{R}\rangle$ be an invariantly finitely $L$-presented group and let $H \unlhd G$ be a $\Phi$-invariant normal subgroup with finite index in $G$. Every invariantly $L$-presented group can be considered as an ascendingly $L$-presented group. Thus, we may consider $\mathcal{Q}=\emptyset$. Consider the notation introduced above. As $G$ is invariantly $L$ presented, we have $K^{\sigma} \subseteq K$ for each $\sigma \in \Phi^{*}$. Since the subgroup $H$ is $\Phi$-invariant, we also $(U K)^{\sigma} \subseteq U K$ for each $\sigma \in \Phi^{*}$. Then Lemma 5.24 shows that $\tilde{L}=U K$. Moreover, as $U K \unlhd F$, we have $L=\tilde{L}$ and thus $U K=\tilde{L}=L$. Let $t \in \mathcal{T}$ be given. As $U K \unlhd F$, the map $\delta_{t}: U K \rightarrow U K, g \mapsto t g t^{-1}$ defines an automorphism of $U K$. The Reidemeister rewriting $\tau: U K \rightarrow F(\mathcal{Y})$ is an isomorphism of free groups and therefore the endomorphisms $\Phi \cup\left\{\delta_{t} \mid\right.$ $t \in \mathcal{T}\}$ of $U K$ translate to endomorphisms $\widehat{\Phi} \cup\left\{\widehat{\delta}_{t} \mid t \in \mathcal{T}\right\}$ of the free group $F(\mathcal{Y})$. Consider the invariant finite $L$-presentation

$$
\left\langle\mathcal{Y}|\emptyset| \widehat{\Phi} \cup\left\{\widehat{\delta}_{t} \mid t \in \mathcal{T}\right\} \mid\{\tau(r) \mid r \in \mathcal{R}\}\right\rangle .
$$

In order to prove that the finite $L$-presentation in Eq. (D.8) defines the subgroup $H$, it suffices to prove that each relation of the presentation in Eq. (D.7) is a consequence of the relations of the $L$-presentation in Eq. (D.8) and vice versa. For $t \in \mathcal{T}, r \in \mathcal{R}$, and $\sigma \in \Phi^{*}$, we consider the relation $\tau\left(t r^{\sigma} t^{-1}\right)$ of the group presentation in Eq. (D.7). Clearly, this relation is a relation in the finite $L$-presentation in Eq. (D.8) because there exists $\widehat{\sigma} \in \widehat{\Phi}^{*}$ so that $(\tau(r))^{\widehat{\sigma}}=\tau\left(r^{\sigma}\right)$. Then $(\tau(r))^{\widehat{\sigma} \delta_{t}}=\tau\left(t r^{\sigma} t^{-1}\right)$. On the other hand, consider the relation $\tau(r)^{\widehat{\sigma}}$ of the finite $L$-presentation in Eq. (D.8) where $r \in \mathcal{R}$ and $\widehat{\sigma} \in\left(\widehat{\Phi} \cup\left\{\widehat{\delta}_{t} \mid t \in \mathcal{T}\right\}\right)^{*}$. Write $\Psi=\widehat{\Phi} \cup\left\{\widehat{\delta}_{t} \mid t \in \mathcal{T}\right\}$. Since $1 \in \mathcal{T}$ and id $\in \Phi^{*}$, we can write each image of an element $\widehat{\delta} \in \Psi$ as $\tau(g)^{\widehat{\delta}}=\tau\left(t g^{\delta} t^{-1}\right)$ for some $t \in \mathcal{T}$ and $\delta \in \Phi^{*}$ where $t$ or $\delta$ is possibly trivial. Since $\widehat{\sigma} \in \Psi^{*}$, we can write $\widehat{\sigma}=\widehat{\sigma}_{1} \cdots \widehat{\sigma}_{n}$ with each $\widehat{\sigma}_{i} \in \Psi$. The image $\tau(r)^{\widehat{\sigma}}$ has the form

$$
\tau(r)^{\widehat{\sigma}}=\tau\left(t_{n} \cdots t_{2}^{\sigma_{3} \cdots \sigma_{n}} t_{1}^{\sigma_{2} \sigma_{3} \cdots \sigma_{n}} \cdot r^{\sigma_{1} \sigma_{2} \cdots \sigma_{n}} \cdot t_{1}^{-\sigma_{2} \sigma_{3} \cdots \sigma_{n}} t_{2}^{-\sigma_{3} \cdots \sigma_{n}} \cdots t_{n}^{-1}\right) .
$$

Since $\mathcal{T}$ is a transversal for $U K$ in $F$, we can write $t_{n} \cdots t_{2}^{\sigma_{3} \cdots \sigma_{n}} t_{1}^{\sigma_{2} \sigma_{3} \cdots \sigma_{n}}=u t$ with $t \in \mathcal{T}$ and $u \in U K$. This yields that $\tau(r)^{\widehat{\sigma}}=\tau\left(u t r^{\sigma_{1} \sigma_{2} \cdots \sigma_{n}} t^{-1} u^{-1}\right)=$ $\tau(u) \tau\left(t r^{\sigma_{1} \sigma_{2} \cdots \sigma_{n}} t^{-1}\right) \tau(u)^{-1}$, which is a consequence of $\tau\left(t r^{\sigma_{1} \sigma_{2} \cdots \sigma_{n}} t^{-1}\right)$. The latter relation $\tau\left(t r^{\sigma_{1} \sigma_{2} \cdots \sigma_{n}} t^{-1}\right)$ is a relation of the group presentation in Eq. (D.7). In summary, each relation of the group presentation in Eq. (D.7) is a consequence of the finite $L$-presentation in Eq. (D.8) and vice versa. 
In order to prove Reidemeister-Schreier Theorem 1.1 for finitely $L$-presented groups, we need to consider finite index subgroups that are not normal. For this purpose, we need to construct the relations $\tau\left(t r^{\sigma} t^{-1}\right)$ with $t \in \mathcal{T}, r \in \mathcal{R}$, and $\sigma \in \Phi^{*}$ in Eq. (D.7). The overall strategy in this paper is to construct the relations as iterated images of the form $\tau\left(s r s^{-1}\right)^{\widehat{\sigma}}$ for $s \in \mathcal{T}$ and some $\widehat{\sigma} \in \widehat{\Phi}^{*}$. If the subgroup $H$ is normal as in Proposition 6.34, the conjugation action $\delta_{t}: U K \rightarrow U K$ enables us to first construct the image $\tau\left(r^{\sigma}\right)=\tau(r)^{\widehat{\sigma}}$ and then to consider the conjugates $\tau\left(r^{\sigma}\right)^{\widehat{\delta}_{t}}=\tau\left(t r^{\sigma} t^{-1}\right)$. However, in general, it is not sufficient to take as iterated relations those $\tau\left(t r t^{-1}\right)^{\sigma}=\tau\left(t^{\sigma} r^{\sigma} t^{-\sigma}\right)$, with $t \in \mathcal{T}$ and $r \in \mathcal{R}$, as $\sigma$ may not be invertible over $\left\{t^{\sigma} \mid t \in \mathcal{T}\right\}$. More precisely, we have the following

Remark 6.33 Let $H=\left\langle a, b^{2}, b a^{3} b^{-1}, b a b^{-2} a^{-1} b^{-1}, b a^{-1} b^{-2} a b^{-1}\right\rangle$ be a subgroup of the Basilica group $G$. The subgroup $H$ is $\sigma$-invariant and thus we can consider the iterated images $\left\{\tau(r)^{\widehat{\sigma}} \mid r \in \mathcal{R}, \sigma \in \Phi^{*}\right\}$. A Schreier transversal $\mathcal{T}$ for $H$ in $G$ is given by $\mathcal{T}=$ $\left\{1, b, b a, b a^{2}, b a b, b a^{2} b\right\}$. We have $\mathcal{T}^{\sigma}=\left\{1, a, a b^{2}, a b^{4}, a b^{2} a, a b^{4} a\right\}$. Note that $\mathcal{T}^{\sigma} \subseteq U K$ holds. Thus we cannot ensure that the iterated images $\left\{\tau\left(t r t^{-1}\right)^{\widehat{\sigma}} \mid r \in \mathcal{R}, t \in \mathcal{T}, \sigma \in \Phi^{*}\right\}$ contain all relations in $E q$. (D.7). As the subgroup $H$ is not normal in $G$, we cannot consider the conjugate action as well. However, an invariant finite L-presentation for the subgroup $H$ can be computed with Theorem 7.35 as the subgroup $H$ is leaf-invariant (see Section D.7 below).

In the following, we use Theorem 6.32 to prove our variant of the Reidemeister-Schreier Theorem for invariantly finitely $L$-presented groups first.

Proposition 6.34 Every finite index subgroup of an invariantly finitely L-presented group is finitely L-presented.

Proof. Let $H$ be a finite index subgroup of an invariantly finitely $L$-presented group $G=$ $F / K$. By Corollary [5.28, the subgroup $H \cong U K / K$ contains a normal subgroup $L / K$ with finite index in $G$ that is $\Phi$-invariant. By Theorem 6.32, the subgroup $L / K \leq F / K$ is finitely $L$-presented. The subgroup $H$ is a finite extension of a finitely $L$-presented group and thus, by Corollary [2.6, the subgroup $H$ is finitely $L$-presented.

Recall that we do not have a method to construct an invariant $L$-presentation for a finite extension of an invariantly $L$-presented group. Therefore, we cannot ensure invariance of the finite $L$-presentation obtained from Corollary 5.28, In Section D.7, we study conditions on a subgroup of an invariantly $L$-presented group that ensure the invariance of the subgroup $L$-presentation. First, we complete our proof of Theorem 1.1;

Proof of Theorem 1.1. Let $G=\langle\mathcal{X}|\mathcal{Q}| \Phi \mid \mathcal{R}\rangle$ be a finitely $L$-presented group and let $H$ be a finite index subgroup of $G$. Denote the free group over $\mathcal{X}$ by $F$. Define the normal subgroups $K=\left\langle\mathcal{Q} \cup \bigcup_{\sigma \in \Phi^{*}} \mathcal{R}^{\sigma}\right\rangle^{F}$ and $M=\left\langle\bigcup_{\sigma \in \Phi^{*}} \mathcal{R}^{\sigma}\right\rangle^{F}$. Let $U \leq F$ be generated by the generators of $H$ so that $H \cong U K / K$ holds. Then we have $M \unlhd K \unlhd F$ and $G=F / K$. Further, the group $J=F / M$ is invariantly finitely $L$-presented by $\langle\mathcal{X}|\emptyset| \Phi \mid \mathcal{R}\rangle$ and it naturally maps onto $G$. The subgroup $U K / M \leq F / M$ has finite index in $J$ as $[F: U K]$ is finite. By Proposition [6.34, the subgroup $U K / M$ of the invariantly finitely 
$L$-presented group $J=F / M$ is finitely $L$-presented. The exact sequence $1 \rightarrow K / M \rightarrow$ $U K / M \rightarrow U K / K \rightarrow 1$ yields that $H \cong U K / K \cong(U K / M) /(K / M)$ where the kernel $K / M$ is finitely generated as a normal subgroup by the image of the fixed relations in $\mathcal{Q}$. By Proposition 2.9, $H$ is finitely $L$-presented as a factor group of the finitely $L$-presented group $U K / M$ whose kernel is finitely generated as a normal subgroup.

\section{D.7 Invariant Subgroup $L$-Presentations}

The algorithms in [9,66] are much more efficient on invariant $L$-presentations. Therefore, we study conditions on a subgroup of an invariantly $L$-presented group to be invariantly $L$-presented itself. By Theorem 6.32, each $\Phi$-invariant normal subgroup $H$ of an invariantly finitely $L$-presented group $G=\langle\mathcal{X}|\mathcal{Q}| \Phi \mid \mathcal{R}\rangle$ is invariantly finitely $L$-presented as soon as $[G: H]$ is finite.

Consider the notion introduced in Section D.6 and let $\pi: F \rightarrow \operatorname{Sym}(U K \backslash F)$ be a permutation representation as usual. Recall that the subgroup $H$ is leaf-invariant, if the $\pi$-leafs

$$
\Psi=\{\psi \delta \mid \psi \in \Phi, \delta \in \mathcal{V}, \psi \delta \notin \mathcal{V}, \psi \delta \pi=\pi\},
$$

of $\mathcal{V}$ satisfy $\Psi=\{\psi \delta \mid \psi \in \Phi, \delta \in \mathcal{V}, \psi \delta \notin \mathcal{V}\}$. This definition yields the following

Theorem 7.35 Each leaf-invariant, finite index subgroup of an invariantly finitely Lpresented group is invariantly finitely L-presented.

Proof. Let $G=\langle\mathcal{X}|\mathcal{Q}| \Phi \mid \mathcal{R}\rangle$ be invariantly finitely $L$-presented and let $H \leq G$ be a leaf-invariant finite index subgroup of $G$. Clearly, we can consider $\mathcal{Q}=\emptyset$ in the following. The $\pi$-leafs $\Psi$ satisfy $\Psi=\{\psi \delta \mid \psi \in \Phi, \delta \in \mathcal{V}, \psi \delta \notin \mathcal{V}\}$. By Lemma 5.21, each $\pi$-leaf $\psi \delta \in \Psi \subseteq \Phi^{*}$ defines an endomorphism of the subgroup UK. Moreover, Lemma 5.21 shows that each $\sigma \in \Phi^{*}$ can be written as $\sigma=\vartheta \delta$ with $\vartheta \in \mathcal{V}$ and $\delta \in \Psi^{*}$. Consider the finite $L$-presentation

$$
\left\langle\mathcal{Y}|\emptyset|\{\widehat{\psi \delta} \mid \psi \delta \in \Psi\} \mid\left\{\tau\left(t r^{\vartheta} t^{-1}\right) \mid \vartheta \in \mathcal{V}, r \in \mathcal{R}, t \in \mathcal{T}\right\}\right\rangle
$$

where $\mathcal{Y}$ denotes the Schreier generators of $U K, \widehat{\psi \sigma}$ denotes the endomorphism of the free group $F(\mathcal{Y})$ induced by the endomorphisms $\psi \sigma$ of $U K$, and $\mathcal{T}$ is a Schreier transversal for $U K$ in $F$. For $t \in \mathcal{T}, \sigma \in \Phi^{*}$, and $r \in \mathcal{R}$, the relation $\tau\left(t r^{\sigma} t^{-1}\right)$ of the group presentation in Eq. (D.7) can be obtained from the $L$-presentation in Eq. (D.9) as follows: Since each $\sigma \in \Phi^{*}$ can be written as $\sigma=\vartheta \delta$ with $\vartheta \in \mathcal{V}$ and $\delta \in \Psi^{*}$, we claim that the relation $\tau\left(t r^{\sigma} t^{-1}\right)$ is a consequence of the image $\tau\left(t r^{\vartheta} t^{-1}\right)^{\widehat{\delta}}$. The latter image satisfies that $\tau\left(t r^{\vartheta} t^{-1}\right)^{\widehat{\delta}}=\tau\left(t^{\delta} r^{\vartheta \delta} t^{-\delta}\right)=\tau\left(t^{\delta} r^{\sigma} t^{-\delta}\right)$. As $\delta \in \Psi^{*}$, we can write $\delta=\delta_{1} \cdots \delta_{n}$ with each $\delta_{i} \in \Psi$. Recall that $\delta_{i} \pi=\pi$ holds. Thus the right-coset $U K 1$ satisfies that $U K 1 \cdot t^{\delta_{i}}=U K 1 \cdot t=U K t$ and therefore $U K t^{\delta_{1} \cdots \delta_{n}}=U K t$. Hence, there exists $u \in U K$ so that $t^{\delta}=u t$ and we obtain

$$
\tau\left(t r^{\vartheta} t^{-1}\right)^{\widehat{\delta}}=\tau\left(t^{\delta} r^{\sigma} t^{-\delta}\right)=\tau\left(u t r^{\sigma} t^{-1} u^{-1}\right)=\tau(u) \tau\left(t r^{\sigma} t^{-1}\right) \tau(u)^{-1}
$$


which is a consequence of $\tau\left(t r^{\sigma} t^{-1}\right)$ and vice versa. Similarly, every relation of the $L$ presentation in Eq. (D.9) is a consequence of the relations in Eq. (D.7). Therefore, the invariant finite $L$-presentation in Eq. (D.9) defines the leaf-invariant finite index subgroup $H$.

For finite $L$-presentations $\langle\mathcal{X}|\mathcal{Q}| \Phi \mid \mathcal{R}\rangle$ with $\Phi=\{\sigma\}$, the leaf-invariance of the subgroup $H$ yields the existence of a positive integer $j$ so that $\sigma^{j} \pi=\pi$ holds. If we assume the positive integer $j$ to be minimal, then $\mathcal{V}=\left\{\right.$ id $\left., \sigma, \ldots, \sigma^{j-1}\right\}$ and $\Psi=\left\{\sigma^{j}\right\}$. In this case, the invariant finite $L$-presentation in Eq. (D.9) becomes

$$
H \cong\left\langle\mathcal{Y}|\emptyset|\left\{\widehat{\sigma^{j}}\right\} \mid\left\{\tau\left(t r^{\sigma^{i}} t^{-1}\right) \mid t \in \mathcal{T}, r \in \mathcal{R}, 0 \leq i<j\right\}\right\rangle .
$$

Note that the subgroup $H$ in Theorem 7.35 is not necessarily normal in $G$. However, leaf-invariance of a subgroup is a restrictive condition on the subgroup. We try to weaken this condition with the following

Definition 7.36 Let $G=\langle\mathcal{X}|\mathcal{Q}| \Phi \mid \mathcal{R}\rangle$ be a finitely L-presented group and let $H \leq G$ be a finite index subgroup with permutation representation $\pi$. The subgroup $H$ is weakly leaf-invariant, if

$$
\Psi=\left\{\psi \delta \mid \psi \in \Phi, \delta \in \tilde{\mathcal{V}}, \psi \delta \notin \tilde{\mathcal{V}}, \psi \delta \sim_{\pi} \mathrm{id}\right\}
$$

satisfies $\Psi=\{\psi \delta \mid \psi \in \Phi, \delta \in \tilde{\mathcal{V}}, \psi \delta \notin \tilde{\mathcal{V}}\}$.

The notion of a weakly leaf-invariant subgroup is less restrictive than leaf-invariance as the low-index subgroups of the Basilica group suggest: Among the 4956 low-index subgroups of the Basilica group with index at most 20 there are 2539 weakly leaf-invariant subgroups; only 156 of these subgroups are leaf-invariant. More precisely, Table D.1 shows the number of subgroups $(\leq)$ that are normal $(\unlhd)$, maximal (max), leaf-invariant (l.i.), weakly leaf-invariant (w.l.i.), and the number of subgroups that are weakly leafinvariant and normal $(\unlhd+$ w.l.i.). For finite $L$-presentations $\langle\mathcal{X}|\mathcal{Q}| \Phi \mid \mathcal{R}\rangle$ with $\Phi=\{\sigma\}$, each leaf-invariant subgroup is weakly leaf-invariant by Lemma 5.26, (iii). On the other hand, a weakly leaf-invariant subgroup with $\Phi=\{\sigma\}$ such that id $\sim_{\pi} \sigma^{\ell}$ holds, is leaf-invariant by Lemma 5.26, (iv). There are subgroups of a finitely $L$-presented group that are weakly leaf-invariant but not leaf-invariant; see Lemma 5.26, (v). If $\Phi$ contains more than one generator, we may ask the following

Question 7.37 Is every leaf-invariant subgroup weakly leaf-invariant?

The problem is that Definitions 5.20 and 7.36 depend on the minimal sets $\mathcal{V}$ and $\tilde{\mathcal{V}}$ which satisfy $\tilde{\mathcal{V}} \subseteq \mathcal{V}$ but which may differ in general. We do not have an answer to this question. Moreover, the sets $\mathcal{V}$ and $\tilde{\mathcal{V}}$ in the Definitions 5.20 and 7.36 may also depend on choice of the ordering $\prec$ in Algorithm D.1, However we have the following

Lemma 7.38 The conditions leaf-invariance and weak leaf-invariance do not depend on the choice of the ordering $\prec$ in Algorithm D.1. 
Table D.1: Subgroups of the Basilica group with index at most 20.

\begin{tabular}{ccccccc}
\hline index & $\leq$ & $\unlhd$ & $\max$ & l.i. & w.l.i & $\unlhd+$ w.l.i \\
\hline 1 & 1 & 1 & 1 & 1 & 1 & 1 \\
2 & 3 & 3 & 3 & 0 & 3 & 3 \\
3 & 7 & 4 & 7 & 4 & 4 & 4 \\
4 & 19 & 7 & 0 & 0 & 19 & 7 \\
5 & 11 & 6 & 11 & 6 & 6 & 6 \\
6 & 39 & 13 & 0 & 0 & 14 & 12 \\
7 & 15 & 8 & 15 & 8 & 8 & 8 \\
8 & 163 & 19 & 0 & 0 & 139 & 19 \\
9 & 115 & 13 & 9 & 49 & 52 & 13 \\
10 & 83 & 19 & 0 & 0 & 22 & 18 \\
11 & 23 & 12 & 23 & 12 & 12 & 12 \\
12 & 355 & 31 & 0 & 0 & 98 & 28 \\
13 & 27 & 14 & 27 & 14 & 14 & 14 \\
14 & 115 & 25 & 0 & 0 & 30 & 24 \\
15 & 77 & 24 & 0 & 24 & 24 & 24 \\
16 & 1843 & 47 & 0 & 0 & 1531 & 43 \\
17 & 35 & 18 & 35 & 18 & 18 & 18 \\
18 & 1047 & 44 & 0 & 0 & 366 & 40 \\
19 & 39 & 20 & 39 & 20 & 20 & 20 \\
20 & 939 & 45 & 0 & 0 & 158 & 42 \\
\hline
\end{tabular}

Proof. We prove this lemma by constructing the set $\mathcal{V}$ returned by Algorithm D.1 (the set $\tilde{\mathcal{V}}$ from Lemma 5.25) independently from the ordering $\prec$ provided that the subgroup is (weakly) leaf-invariant. Let $\pi: F \rightarrow \operatorname{Sym}(U K \backslash F)$ be the permutation representation as usual and assume that the subgroup is leaf-invariant. For each $j \geq 0$, we write $\Phi^{(j)}=\left\{\sigma \in \Phi^{*} \mid\|\sigma\|=j\right\}$. Define $\mathcal{W}_{0}=\{\mathrm{id}\}$ and recursively $\mathcal{W}_{n+1}=\left\{\sigma \in \Phi \mathcal{W}_{n} \mid\right.$ $\sigma \pi \neq \pi\} \subseteq \Phi^{(n+1)}$. Let $\mathcal{W}=\bigcup_{n>0} \mathcal{W}_{n}$. Clearly, the construction of $\mathcal{W}$ does not depend on the ordering $\prec$ in Algorithm D.1. We show that the sets $\mathcal{W}$ and $\mathcal{V}$ coincide. Write $\mathcal{S}_{j}=\mathcal{V} \cap \Phi^{(j)}$ and $\mathcal{T}_{j}=\mathcal{W} \cap \Phi^{(j)}$. Then $\mathcal{S}_{0}=\{\operatorname{id}\}=\mathcal{T}_{0}$. In order to prove that $\mathcal{W}=\mathcal{V}$ holds, it suffices to show that $\mathcal{S}_{j}=\mathcal{T}_{j}$ for each $j \geq 0$. Suppose that, for $n \in \mathbb{N}_{0}$, we have $\mathcal{S}_{j}=\mathcal{T}_{j}$ for all $j<n$ while $\mathcal{S}_{n} \neq \mathcal{T}_{n}$. If $\sigma \in \mathcal{S}_{n}=\mathcal{V} \cap \Phi^{(n)}$, it is contained in $\mathcal{V}$ and hence it satisfies $\sigma \pi \neq \pi$. Moreover, we have $\sigma \in \Phi \mathcal{S}_{n-1}=\Phi \mathcal{T}_{n-1}$ and thus $\sigma \in \mathcal{T}_{n}$. If $\sigma \in \mathcal{T}_{n}=\mathcal{W} \cap \Phi^{(n)}$ but $\sigma \notin \mathcal{S}_{n}$, then $\sigma=\psi \delta$ with $\psi \in \Phi$ and $\delta \in \mathcal{T}_{n-1}=\mathcal{S}_{n-1} \subseteq \mathcal{V}$. Note that $\sigma$ satisfies $\sigma=\psi \delta$ with $\delta \in \mathcal{V}, \psi \in \Phi$, and $\sigma=\psi \delta \notin \mathcal{V}$. Hence $\sigma$ is a $\pi$-leaf. Since the subgroup $H$ is leaf-invariant we have $\sigma \pi=\pi$. This is a contradiction to $\sigma \in \mathcal{T}_{n}$.

For proving the statement for weak leaf-invariance, the same arguments as above and the construction $\tilde{\mathcal{S}}_{0}=\{\operatorname{id}\}$ and $\tilde{\mathcal{S}}_{n}=\left\{\sigma \in \Phi \tilde{\mathcal{S}}_{n} \mid \sigma \not_{\pi_{\pi}}\right.$ id $\}$ apply. 
The subgroup $J=\left\langle x_{1}, x_{2}, x_{3}, x_{4} x_{1} x_{4}^{-1}, x_{4}^{3}\right\rangle$ of the subgroup $H$ in Section D.4 is weakly leaf-invariant but it is not leaf-invariant. The notion of a weakly leaf-invariant subgroup yields the following

Lemma 7.39 A normal subgroup $U K \unlhd F$ is $\sigma$-invariant if and only if $\sigma \sim_{\pi}$ id.

Proof. $\quad$ Since $U K \unlhd F$, we have $U K=\operatorname{Core}_{F}(U K)=\operatorname{ker}(\pi)$. Thus $\operatorname{im}(\pi) \cong F / \operatorname{ker}(\pi)=$ $F / U K$. If $U K$ is $\sigma$-invariant, then $\sigma$ induces an endomorphism $\bar{\sigma}: F / U K \rightarrow F / U K$ and, as $F / U K \cong \operatorname{im}(\pi)$, it induces an endomorphism $\gamma: \operatorname{im}(\pi) \rightarrow \operatorname{im}(\pi)$ so that the diagram

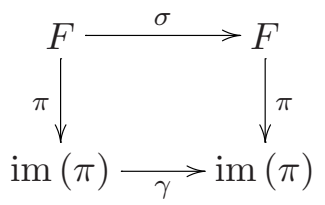

commutes. Thus $\sigma \sim_{\pi}$ id. If, on the other hand, $\sigma \pi=\pi \gamma$ holds for a homomorphism $\gamma: \operatorname{im}(\pi) \rightarrow \operatorname{im}(\sigma \pi)$, each $g \in U K=\operatorname{ker}(\pi)$ satisfies $1=1^{\gamma}=\left(g^{\pi}\right)^{\gamma}=g^{\pi \gamma}=g^{\sigma \pi}=$ $\left(g^{\sigma}\right)^{\pi}$. Hence $g^{\sigma} \in \operatorname{ker}(\pi)=U K$ and thus, $U K$ is $\sigma$-invariant.

Lemma 7.39 yields that a $\Phi$-invariant normal subgroup is weakly leaf-invariant. However, there exist subgroups which are weakly leaf-invariant but not $\Phi$-invariant (e.g. the subgroup $H=\left\langle a, b a b^{-1}, b^{3}\right\rangle$ of the Basilica group in Section D.4 satisfies $\sigma^{2} \sim_{\pi}$ id but not $\sigma \gg_{\pi}$ id; thus, it is weakly leaf-invariant but not $\Phi$-invariant). The condition $U K \unlhd F$ in Lemma 7.39 is necessary, as we have the following

Remark 7.40 The condition $U K \unlhd F$ in Lemma 7.39 is necessary, as the subgroup $H=\left\langle a, b^{2}, b a^{3} b^{-1}, b a b^{-2} a^{-1} b^{-1}, b a^{-1} b^{-2} a b^{-1}\right\rangle$ of the Basilica group $G$ is not normal in $G$, it satisfies $(U K)^{\sigma} \subseteq U K$; however, it does not satisfy $\sigma \sim_{\pi}$ id.

On the other hand, the subgroup $H=\left\langle a, b a b, b a^{-1} b, b^{4}\right\rangle$ of the Basilica group $G$ satisfies $\sigma \leadsto \pi$ id but it does not satisfy $(U K)^{\sigma} \subseteq U K$ as $\left[F: \operatorname{Core}_{F}(U K)\right]=[F: \tilde{L}]=8 \neq$ $4=[F: U K]$.

A weakly leaf-invariant normal subgroup satisfies the following variant of our Reidemeister-Schreier Theorem:

Theorem 7.41 A weakly leaf-invariant normal subgroup which has finite index in an invariantly finitely L-presented group is invariantly finitely L-presented.

Proof. Let $G=\langle\mathcal{X}|\mathcal{Q}| \Phi \mid \mathcal{R}\rangle$ be invariantly finitely $L$-presented and let $H \cong U K / K$ be a finite index normal subgroup of $G$. As usual, we may consider $\mathcal{Q}=\emptyset$ as $G$ is invariantly $L$-presented. Let $\tilde{\mathcal{V}} \subseteq \mathcal{V}$ be the set from Lemma 5.25. Since $H$ is weakly leafinvariant, the weak-leafs $\Psi$ in Definition 7.36 satisfy $\Psi=\{\psi \delta \mid \psi \in \Phi, \delta \in \tilde{\mathcal{V}}, \psi \delta \notin \tilde{\mathcal{V}}\}$. By Lemma 7.39, each $\psi \delta \in \Psi$ induces an endomorphism of the normal subgroup $U K \unlhd F$. Let $\mathcal{T}$ be a Schreier transversal for $U K$ in $F$ and let $\mathcal{Y}$ denote the Schreier generators of the subgroup $U K$. Then each endomorphism $\psi \delta \in \Psi$ of $U K$ translates to an endomorphism $\widehat{\psi \delta}$ of the free group $F(\mathcal{Y})$. Consider the invariant finite $L$-presentation

$$
\left\langle\mathcal{Y}|\emptyset|\{\widehat{\psi \delta} \mid \psi \delta \in \Psi\} \cup\left\{\widehat{\delta}_{t} \mid t \in \mathcal{T}\right\} \mid\left\{\tau\left(r^{\sigma}\right) \mid r \in \mathcal{R}, \sigma \in \tilde{\mathcal{V}}\right\}\right\rangle,
$$


where $\delta_{t}$ denotes the endomorphism of $U K$ which is induced by conjugation by $t \in \mathcal{T}$. The finite $L$-presentation in Eq. (D.10) defines the normal subgroup $H$. This statement follows with the same techniques as above; in particular, it follows from rewriting the presentation in Eq. (D.7).

The subgroup $H$ in Section D.4 is a normal subgroup satisfying $\sigma^{2} \sim_{\pi}$ id. Hence, Theorem 7.41 shows that this subgroup is invariantly finitely $L$-presented. Even noninvariant $L$-presentations may give rise to invariant subgroup $L$-presentations as the following shows:

Remark 7.42 There are non-invariant L-presentation $G=\langle\mathcal{X}|\mathcal{Q}| \Phi \mid \mathcal{R}\rangle$ and finite index subgroups $H \leq G$ that satisfy $(U K)^{\sigma} \subseteq U K$ for each $\sigma \in \Phi^{*}$. For instance, the finite L-presentation of Baumslag's group $G$ in 64 is non-invariant (see the proof of Proposition (2.2) while its index-3 subgroup $H=\left\langle a^{3}, b, t\right\rangle$ satisfies $(U K)^{\sigma} \subseteq U K$ for each $\sigma \in \Phi$. The subgroup $H$ even admits an invariant $L$-presentation over the generators $x=a^{3}$ and $y=a^{2} t a^{-2}$ given by

$$
\left\langle\{x, y\}|\emptyset|\left\{\delta_{t}, \delta_{t^{2}}\right\} \mid\left\{y^{-1} x y x^{-4}\right\}\right\rangle
$$

where $\delta_{t}$ is induced by the map $x \mapsto x$ and $y \mapsto x y x^{-3}$ and $\delta_{t^{2}}$ is induced by the map $x \mapsto x$ and $y \mapsto x y x^{-2}$.

The finite $L$-presentations for finite index subgroups in Proposition 6.34, Theorem 7.35, and Theorem 7.41, are derived from the group's $L$-presentation $\langle\mathcal{X}|\mathcal{Q}| \Phi \mid \mathcal{R}\rangle$ by restricting to those endomorphisms in $\Phi^{*}$ which restrict to the subgroup. However, there are subgroups of an invariantly $L$-presented group so that no endomorphism from $\Phi^{*}$ restricts to the subgroup. In this case the finite $L$-presentation for the finite index subgroup needs to be constructed as a finite extension of the finitely $L$-presented stabilizing core $L$ as in the proof of Theorem 1.1. The following remark gives an example of a subgroup of the invariantly finitely $L$-presented Basilica group so that no endomorphism from $\Phi^{*}$ restricts to the subgroup:

Remark 7.43 Let $H=\left\langle b^{2}, a^{3}, a b^{2} a^{-1}, a^{-1} b^{2} a, b a b^{-1} a\right\rangle$ denote a subgroup of the Basilica group $G$. Then $H$ is a normal subgroup with index 6 in $G$. We are not able to find an invariant finite L-presentation for $H$.

The subgroup $H$ admits the permutation representation $\pi: F \rightarrow \operatorname{Sym}(U K \backslash F)$. We have

$$
\pi:\left\{\begin{aligned}
a & \mapsto(1,2,3)(4,6,5) \\
b & \mapsto(1,4)(2,5)(3,6)
\end{aligned} \quad \text { and } \quad \sigma \pi:\left\{\begin{aligned}
a & \mapsto() \\
b & \mapsto(1,2,3)(4,6,5)
\end{aligned}\right.\right.
$$

as well as

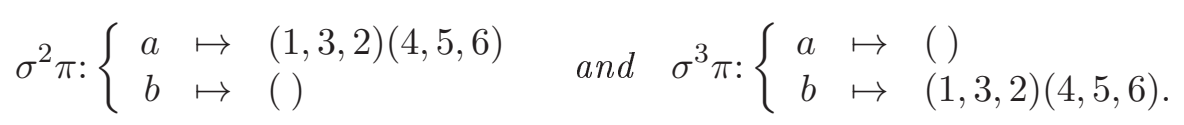

Clearly, $\sigma^{3} \sim_{\pi} \sigma$ but, for each $0<\ell<3$, we do not have $\sigma^{\ell} \leadsto_{\pi}$ id. The homomorphism $\gamma: \operatorname{im}(\sigma \pi) \rightarrow \operatorname{im}\left(\sigma^{3} \pi\right)$ with $\sigma^{3} \pi=\sigma \pi \gamma$ is bijective. Suppose there existed $\sigma^{n} \in \Phi^{*}$ so 
that the subgroup UK is $\sigma^{n}$-invariant. By Lemma 7.39, the normal subgroup UK is $\sigma^{n}$ invariant if and only if $\sigma^{n} \sim_{\pi}$ id holds. Clearly $n>3$. Since $\sigma^{n} \sim_{\pi}$ id, there exists a homomorphism $\psi: \operatorname{im}(\pi) \rightarrow \operatorname{im}\left(\sigma^{n} \pi\right)$ so that $\sigma^{n} \pi=\pi \psi$. We obtain $\pi \psi=\sigma^{n} \pi=$ $\sigma^{n-3} \sigma^{3} \pi=\sigma^{n-3} \sigma \pi \gamma=\sigma^{n-2} \pi \gamma$. Iterating this rewriting process eventually yields a positive integer $0 \leq \ell<3$ so that $\pi \psi=\sigma^{n} \pi=\sigma^{\ell} \pi \gamma^{m}$ for some $m \in \mathbb{N}$. As $\gamma$ is bijective, this yields that $\sigma^{\ell} \pi=\pi \psi \gamma^{-m}$ and hence $\sigma^{\ell} \leadsto \pi \mathrm{id}$; a contradiction. Thus there is no positive integer $n \in \mathbb{N}$ so that $\sigma^{n} \sim_{\pi} \mathrm{id}$. Hence, no substitution in $\Phi^{*}$ restricts to the subgroup UK.

Our method to compute a finite $L$-presentation for the subgroup $H$ in Remark 7.43 is therefore given by our explicit proof of Theorem 1.1. If the subgroup $H$ in Remark 7.43 admits an invariant finite $L$-presentation, the substitutions may not be related to the substitutions $\Phi$ of the finite $L$-presentation $\langle\mathcal{X}|\mathcal{Q}| \Phi \mid \mathcal{R}\rangle$ of the Basilica group in Proposition 4.13. It is neither clear to us whether $H$ admits an invariant finite $L$ presentation at all nor do we know how to possibly prove that $H$ does not admit such invariant finite $L$-presentation.

\section{D.8 Examples of Subgroup $L$-Presentations}

In this section, we consider the subgroup $H=\left\langle a, b a b^{-1}, b^{3}\right\rangle$ of the Basilica group $G$ as in Section D.4. We demonstrate how our methods apply to this subgroup and, in particular, how to compute the $L$-presentation in Section D.4.

Coset-enumeration for finitely $L$-presented groups [67] allows us to compute the permutation representation $\pi: F \rightarrow \operatorname{Sym}(U K \backslash F)$ for the group's action on the right-cosets. A Schreier transversal for $H$ in $G$ is given by $\mathcal{T}=\left\{1, b, b^{2}\right\}$ and we have

$$
\pi: F \rightarrow \mathcal{S}_{n},\left\{\begin{aligned}
a & \mapsto() \\
b & \mapsto(1,2,3) .
\end{aligned}\right.
$$

Moreover, $H$ is a normal subgroup with index 3 in $G$ and it satisfies $\sigma^{2} \sim_{\pi}$ id. By Lemma 5.26, there exists an integer $k \geq 2$ so that $\sigma^{k} \sim \mathrm{id}$; we can verify that $\sigma^{4} \pi=\pi$ holds. Thus $\sigma^{4} \sim$ id. In particular, the subgroup $H$ is (weakly) leaf-invariant and normal. Therefore the following techniques apply to this subgroup:

- As the subgroup $H$ is a finite index subgroup of an invariantly finitely $L$-presented group $G$, the general methods of Proposition 6.34 and Theorem 6.32 apply.

- As the subgroup $H$ is leaf-invariant, the methods in Theorem 7.35 apply.

- As the subgroup $H$ is weakly leaf-invariant and normal, the methods in Theorem 7.41 apply.

We demonstrate these different techniques for the subgroup $H$. First, we consider the general method from Proposition 6.34. Note that the stabilizing subgroup $L$ and stabilizing core $\tilde{L}$ coincide by Corollary 5.30. The stabilizing subgroups $L=\tilde{L}$ have index 9 
in $F$ and a Schreier generating set for $L=\tilde{L}$ is given by

$$
\begin{array}{lll}
x_{1}=a^{3} & x_{4}=a b a b^{-1} a^{-2} & x_{7}=a^{2} b a b^{-1} \\
x_{2}=b a b^{-1} a^{-1} & x_{5}=a b^{2} a^{-1} b^{-2} & x_{8}=a^{2} b^{2} a^{-2} b^{-2}
\end{array} \quad x_{10}=b^{2} a^{2} b a^{-2} .
$$

Let $F$ denote the free group over $\{a, b\}$ and let $E$ denote the free group over $\left\{x_{1}, \ldots, x_{10}\right\}$. The Reidemeister rewriting $\tau: F \rightarrow E$ allows us to rewrite the iterated relation $r=\left[a, a^{b}\right]$. We obtain $\tau(r)=x_{1}^{-1} x_{10}^{-1} x_{6} x_{10}^{-1} x_{9} x_{3}$. Furthermore, the rewriting $\tau$ allows us to translate the substitution $\sigma$ of the Basilica group to an endomorphism of the free group $E$. The homomorphism $\widehat{\sigma}: E \rightarrow E$ is induced by the map

$$
\begin{array}{rlrl}
x_{1} & \mapsto x_{3}^{2}, & x_{6} & \mapsto x_{8} x_{9}, \\
x_{2} & \mapsto x_{5}, & \mapsto x_{3} x_{2} x_{5} x_{6}, \\
x_{3} & \mapsto x_{1}, & \mapsto x_{3} x_{2} x_{4} x_{10}^{-1} x_{8}^{-1}, \\
x_{4} & \mapsto x_{6} x_{2}^{-1} x_{3}^{-1}, & x_{9} & \mapsto x_{8} x_{10} x_{8} x_{10}, \\
x_{5} & \mapsto x_{8}^{-1}, & x_{10} & \mapsto x_{8} x_{10} x_{7} x_{3}^{-1} .
\end{array}
$$

Similarly, the conjugation actions $\delta_{a}$ and $\delta_{b}$ which are induced by conjugation with $a$ and $b$, respectively, translate to endomorphisms $\widehat{\delta}_{a}$ and $\widehat{\delta}_{b}$ of the free group $E$. By Proposition 6.34, the stabilizing subgroups $L=\tilde{L}$ are finitely $L$-presented by

$$
M=L / K \cong\left\langle\left\{x_{1}, \ldots, x_{10}\right\}|\emptyset|\left\{\widehat{\sigma}, \widehat{\delta}_{a}, \widehat{\delta}_{b}\right\} \mid\left\{x_{1}^{-1} x_{10}^{-1} x_{6} x_{10}^{-1} x_{9} x_{3}\right\}\right\rangle .
$$

The subgroup $H$ satisfies the short exact sequence $1 \rightarrow M \rightarrow H \rightarrow \mathbb{Z}_{3} \rightarrow 1$ with a cyclic group $\mathbb{Z}_{3}=\left\langle\alpha \mid \alpha^{3}=1\right\rangle$ of order 3. Corollary 2.6 yields the following finite $L$-presentation for the subgroup $H$ :

$$
\left\langle\left\{\alpha, x_{1}, \ldots, x_{10}\right\}\left|\left\{\alpha^{3} x_{1}^{-1}\right\} \cup\left\{\left(x_{i}^{-1}\right)^{\alpha} x_{i}^{\delta_{a}}\right\}_{1 \leq i \leq 10}\right| \tilde{\Psi} \mid\left\{x_{1}^{-1} x_{10}^{-1} x_{6} x_{10}^{-1} x_{9} x_{3}\right\}\right\rangle .
$$

where the substitutions $\widehat{\Psi}=\left\{\widehat{\sigma}, \widehat{\delta}_{a}, \widehat{\delta}_{b}\right\}$ of $M$ 's finite $L$-presentation are dilated to endomorphisms $\tilde{\Psi}=\left\{\widetilde{\sigma}, \widetilde{\delta}_{a}, \widetilde{\delta}_{b}\right\}$ of the free group over $\left\{\alpha, x_{1}, \ldots, x_{10}\right\}$ as in the proof of Proposition 2.5 .

Secondly, the subgroup $H$ is (weakly) leaf-invariant and normal. Therefore, the methods in Section D.7 apply. First, we consider the construction in Theorem 7.35 for leaf-invariant subgroups: A Schreier generating set for the subgroup $U K$ is given by $x_{1}=a, x_{2}=b a b^{-1}, x_{3}=b^{2} a b^{-2}$, and $x_{4}=b^{3}$. Since $\sigma^{4} \pi=\pi$, the subgroup $H$ is $\sigma^{4}$-invariant and its suffices to rewrite the relation $r=[a, b]$ and its images $\tau\left(t^{\sigma^{i}} t^{-1}\right)$, $0 \leq i<4$. These have the form:

\begin{tabular}{lccc}
\hline$i$ & $t=1$ & $t=b$ & $t=b^{2}$ \\
\hline 0 & $x_{1}^{-1} x_{4}^{-1} x_{3}^{-1} x_{4} x_{1} x_{4}^{-1} x_{3} x_{4}$ & $x_{2}^{-1} x_{1}^{-1} x_{2} x_{1}$ & $x_{3}^{-1} x_{2}^{-1} x_{3} x_{2}$ \\
1 & $x_{4}^{-1} x_{2}^{-1} x_{4}^{-1} x_{3} x_{4} x_{2}^{-1} x_{4} x_{1}$ & $x_{4}^{-1} x_{3}^{-1} x_{1} x_{3}^{-1} x_{4} x_{2}$ & $x_{1}^{-1} x_{4}^{-1} x_{2} x_{4} x_{1}^{-1} x_{3}$ \\
2 & $x_{1}^{-2} x_{4}^{-1} x_{2}^{-2} x_{4} x_{1}^{2} x_{4}^{-1} x_{2}^{2} x_{4}$ & $x_{2}^{-2} x_{4}^{-1} x_{3}^{-2} x_{4} x_{2}^{2} x_{4}^{-1} x_{3}^{2} x_{4}$ & $x_{3}^{-2} x_{1}^{-2} x_{3}^{2} x_{1}^{2}$ \\
3 & $x_{4}^{-2} x_{3}^{-2} x_{4}^{-1} x_{2}^{2} x_{4} x_{3}^{-2} x_{4}^{2} x_{1}^{2}$ & $x_{4}^{-1} x_{1}^{-2} x_{4}^{-2} x_{3}^{2} x_{4}^{2} x_{1}^{-2} x_{4} x_{2}^{2}$ & $x_{4}^{-1} x_{2}^{-2} x_{4}^{-1} x_{1}^{2} x_{4} x_{2}^{-2} x_{4} x_{3}^{2}$ \\
\hline
\end{tabular}


Denote the set of relations above by $\mathcal{S}$. The endomorphism $\sigma^{4}$ translates, via $\tau$, to an endomorphism of the free group over $\left\{x_{1}, \ldots, x_{4}\right\}$ which is induced by the map

$$
\widehat{\sigma^{4}}:\left\{\begin{aligned}
x_{1} & \mapsto x_{1}^{4} \\
x_{2} & \mapsto x_{4} x_{2}^{4} x_{4}^{-1} \\
x_{3} & \mapsto x_{4}^{2} x_{3}^{4} x_{4}^{-2} \\
x_{4} & \mapsto x_{4}^{4}
\end{aligned}\right.
$$

By Theorem 7.35, an $L$-presentation for the subgroup $H$ is given by

$$
H \cong\left\langle\left\{x_{1}, \ldots, x_{4}\right\}|\emptyset| \widehat{\sigma^{4}}\right\}|\mathcal{S}\rangle .
$$

Finally, the subgroup $H$ is weakly leaf-invariant and normal. Therefore, the methods in Theorem 7.41 apply. As $\sigma^{2} \sim_{\pi}$ id, it suffices to consider the relations $\tau(r), \tau\left(r^{\sigma}\right)$, and their images under the substitutions $\widehat{\sigma^{2}}$ and $\widehat{\delta_{b}}$ (because a Schreier transversal is given by $\left.\mathcal{T}=\left\{1, b, b^{2}\right\}\right)$. The substitutions $\widehat{\sigma^{2}}$ and $\widehat{\delta_{b}}$ are induced by the maps

$$
\widehat{\sigma^{2}}:\left\{\begin{aligned}
x_{1} & \mapsto x_{1}^{2}, \\
x_{2} & \mapsto x_{3}^{2}, \\
x_{3} & \mapsto x_{4} x_{2}^{2} x_{4}^{-1}, \quad \text { and } \quad \widehat{\delta_{b}}:\left\{\begin{array}{rl}
x_{1} & \mapsto x_{2}, \\
x_{4} & \mapsto x_{4}^{2},
\end{array} x_{3} \mapsto x_{3},\right. \\
x_{3} & \mapsto x_{4} x_{4}^{-1}, \\
x_{4} & \mapsto x_{4} .
\end{aligned}\right.
$$

Theorem 7.41 yields the finite $L$-presentation

$$
H \cong\left\langle\left\{x_{1}, \ldots, x_{4}\right\}|\emptyset|\left\{\widehat{\sigma^{2}}, \widehat{\delta_{b}}\right\} \mid\left\{\tau(r), \tau\left(r^{\sigma}\right)\right\}\right\rangle
$$

for the subgroup $H$ as in Section D.4.

\section{D.8.1 An Application to the Grigorchuk Group}

As a finite $L$-presentation of a group allows the application of computer algorithms, we may use our constructive proof of Theorem 1.1 to investigate the structure of a selfsimilar group by its finite index subgroups as in [77]. As an application, we consider the Grigorchuk group, see [53], $\mathfrak{G}=\langle a, b, c, d\rangle$ and its normal subgroup $D=\langle d\rangle^{\mathfrak{G}}$. We show that the subgroup $D=\langle d\rangle^{\mathfrak{G}}$ has a minimal generating set with 8 elements and thereby we correct a mistake in [11, 58$]$.

The Grigorchuk group $\mathfrak{G}$ satisfies the well-known

Proposition 8.44 (Lysënok [96]) The group $\mathfrak{G}$ is invariantly finitely L-presented by $\left.\mathfrak{G} \cong\left\langle\{a, b, c, d\}\left|\left\{a^{2}, b^{2}, c^{2}, d^{2}, b c d\right\}\right|\{\sigma\}\right|\left\{(a d)^{4},(\text { adacac })^{4}\right\}\right\rangle$, where $\sigma$ is the endomorphism of the free group over $\{a, b, c, d\}$ induced by the mapping $a \mapsto a c a, b \mapsto d, c \mapsto b$, and $d \mapsto c$.

It was claimed in [11, Section 4.2] and in [58, Section 6] that the normal subgroup $D=$ $\langle d\rangle^{\mathfrak{G}}$ is generated by $\left\{d, d^{a}, d^{a c}, d^{a c a}\right\}$. In the following, we show that the Reidemeister Schreier Theorem 1.1 allows us to prove that a generating set for $D=\langle d\rangle^{\mathfrak{G}}$ contains 
at least 8 elements. The coset-enumeration for finitely $L$-presented groups [67] and the solution to the subgroup membership problem for finite index subgroups [67] show that the subgroup

$$
H=\left\langle d, d^{a}, d^{a c}, d^{a c a}, d^{a c a c}, d^{a c a c a}, d^{a c a c a c}, d^{a c a c a c a}\right\rangle
$$

has index 16 in $\mathfrak{G}$. It is a normal subgroup of $\mathfrak{G}$ so that $\mathfrak{G} / H$ is a dihedral group of order 16. In particular, the subgroup $H$ and the normal subgroup $D=\langle d\rangle^{\mathfrak{G}}$ coincide. A permutation representation $\pi: F \rightarrow \mathcal{S}_{n}$ for the group's action on the right-cosets $U K \backslash F$ is given by

$$
\pi: F \rightarrow \mathcal{S}_{16},\left\{\begin{aligned}
a & \mapsto(1,2)(3,5)(4,6)(7,9)(8,10)(11,13)(12,14)(15,16) \\
b & \mapsto(1,3)(2,4)(5,7)(6,8)(9,11)(10,12)(13,15)(14,16) \\
c & \mapsto(1,3)(2,4)(5,7)(6,8)(9,11)(10,12)(13,15)(14,16) \\
d & \mapsto() .
\end{aligned}\right.
$$

Our variant of the Reidemeister-Schreier Theorem and the techniques introduced in Section D.7 enable us to compute a subgroup $L$-presentation for $D$. For this purpose, we first note that $\sigma^{3} \sim_{\pi}$ id. Hence, the normal core $D=\operatorname{Core}_{F}(U K)=\operatorname{ker}(\pi)$ is $\sigma^{3}$-invariant. The core $\operatorname{Core}_{F}(U K)$ is a free group with rank 49 and a Schreier transversal for $D$ in $\mathfrak{G}$ is given by

$$
1, a, b, a b, b a, a b a, b a b,(a b)^{2},(b a)^{2}, a(b a)^{2}, b(a b)^{2},(a b)^{3},(b a)^{3}, a(b a)^{3}, b(a b)^{3},(a b)^{4} .
$$

A finite $L$-presentation with generators $d_{0}=d, d_{1}=d^{a}, d_{2}=d^{a c}, d_{3}=d^{a c a}, d_{4}=d^{a c a c}$, $d_{5}=d^{a c a c a}, d_{6}=d^{\text {acacac }}$, and $d_{7}=d^{\text {acacaca }}$ is given by

$$
D \cong\left\langle\left\{d_{0}, \ldots, d_{7}\right\}|\emptyset|\left\{\widehat{\sigma}, \delta_{a}, \delta_{b}\right\} \mid \mathcal{R}\right\rangle,
$$

where the iterated relations are

$$
\mathcal{R}=\left\{d_{0}^{2},\left[d_{1}, d_{0}\right],\left[d_{1}, d_{4}\right],\left[d_{7}, d_{3} d_{4}\right]^{4},\left[d_{7} d_{0}, d_{3} d_{4}\right],\left(d_{3} d_{7} d_{4} d_{0}\right)^{2},\left(d_{7} d_{4}^{d_{3}} d_{0} d_{3}^{d_{4}}\right)^{2}\right\}
$$

and the endomorphisms $\left\{\widehat{\sigma}, \delta_{a}, \delta_{b}\right\}$ are induced by the maps

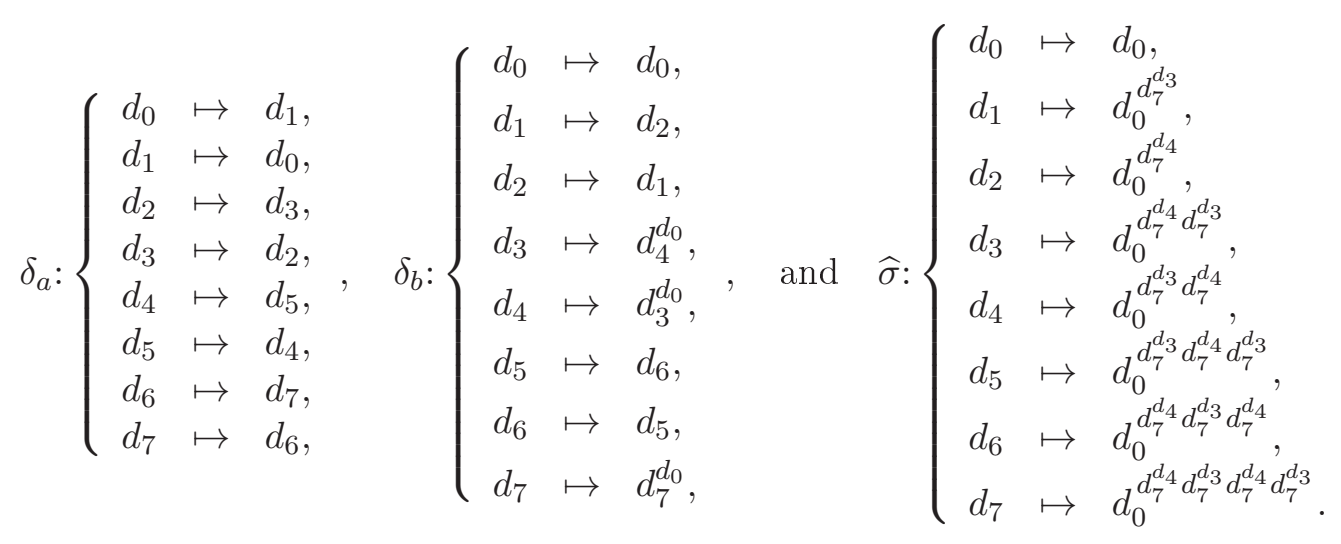

The latter $L$-presentation of the normal subgroup $D$ allows us to compute the abelianization $D /[D, D]$ using the methods from [9]. These computations yield that $D /[D, D] \cong$ 
$\left(\mathbb{Z}_{2}\right)^{8}$ is 2-elementary abelian of rank 8 . Hence, the normal subgroup $D$ has a minimal generating set of length at least 8 . Because a generating set with 8 generators was already given in Eq. (D.11), a minimal generating set of $D$ has precisely 8 elements. In particular, this shows that $D \neq\left\langle d, d^{a}, d^{a c}, d^{a c a}\right\rangle$. The latter mistake could have been detected also by computing the abelianization of the image of $D=\langle d\rangle^{\mathfrak{G}}$ in a finite quotient of $\mathfrak{G}$ (e.g. the quotient $G / \operatorname{Stab}(n)$ for $n \geq 4$ ) by hand or using a computer algebra system such as GAP.

\section{Acknowledgments}

I am grateful to Laurent Bartholdi and to the referee for valuable comments and suggestions.

René Hartung, Mathematisches Institut, Georg-August Universität Zu Göttingen, Bunsenstrasse 3-5, 37073 Göttingen, Germany

Email: rhartung@uni-math.gwdg.de

June 2011 (revised October 2011) 


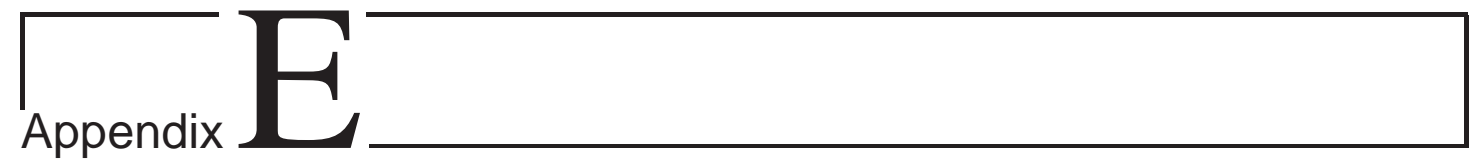

\title{
Approximating the Schur Multiplier of Certain Infinitely Presented Groups via Nilpotent Quotients
}

\begin{abstract}
We describe an algorithm for computing successive quotients of the Schur multiplier $M(G)$ for a group $G$ given by an invariant finite $L$-presentation. As application, we investigate the Schur multipliers of various self-similar groups such as the Grigorchuk super-group, the generalized Fabrykowski-Gupta groups, the Basilica group and the Brunner-Sidki-Vieira group.
\end{abstract}

Keywords. Schur multiplier; recursive presentations; Grigorchuk group; selfsimilar groups;

\section{E.1 Introduction}

The Schur multiplier $M(G)$ of a group $G$ can be defined as the second homology group $H_{2}(G, \mathbb{Z})$. It was introduced by Schur and is, for instance, relevant in the theory of central group extensions. In combinatorial group theory, the Schur multiplier found its applications due to the Hopf formula: if $F$ is a free group and $R$ is a normal subgroup of $F$ so that $G \cong F / R$, then the Schur multiplier of $G$ is isomorphic to the factor group $\left(R \cap F^{\prime}\right) /[R, F]$. For further details on the Schur multiplier we refer to [121, Chapter 11].

The Hopf formula yields that every finitely presentable group has a finitely generated Schur multiplier. This is used in [56] for proving that the Grigorchuk group is not finitely presentable: its Schur multiplier is infinitely generated 2-elementary abelian. This answers the questions in [19] and [127. There are various examples of self-similar groups other than the Grigorchuk group for which it is not known whether their Schur multiplier is finitely generated or whether the groups are finitely presented. 
The first aim of this paper is to introduce an algorithm for investigating the Schur multiplier of self-similar groups with a view towards its finite generation. Let $G$ be a group with a presentation $G \cong F / R$. Then $G / \gamma_{c} G \cong F / R \gamma_{c} F$, where $\gamma_{c} G$ denotes the $c$-th term of the lower central series of $G$. We identify $M(G)$ with $\left(R \cap F^{\prime}\right) /[R, F]$ and $M\left(G / \gamma_{c} G\right)$ with $\left(R \gamma_{c} F \cap F^{\prime}\right) /\left[R \gamma_{c} F, F\right]$ and define

$$
\varphi_{c}: M(G) \rightarrow M\left(G / \gamma_{c} G\right), g[R, F] \mapsto g\left[R \gamma_{c} F, F\right]
$$

Then $\varphi_{c}$ is a homomorphism of abelian groups. We describe an effective method to determine the Dwyer quotients $M_{c}(G)=M(G) / \operatorname{ker} \varphi_{c}$, for $c \in \mathbb{N}$, provided that $G$ is given by an invariant finite $L$-presentation, see [6,9] or Section E.2 below. Every finitely presented group and many self-similar groups can be described by a finite invariant $L$ presentation. An implementation of our algorithm is available in the NQL-package 65] of the computer algebra system GAP; see [50.

We have applied our algorithm to various examples of self-similar groups: the Grigorchuk super-group $\tilde{\mathfrak{G}}$, see [11, the Basilica group $\Delta$, see [60,61], the Brunner-Sidki-Vieira group BSV, see [29], and some generalized Fabrykowski-Gupta groups $\Gamma_{d}$, see [45, 57]. As a result, we observed that the sequence $\left(M_{1}(G), \ldots, M_{c}(G), M_{c+1}(G), \ldots\right)$ exhibits a periodicity in $c$ in all these cases. Based on this, we propose the following conjecture.

\section{Conjecture I}

- $M_{c}(\tilde{\mathfrak{G}})$ is 2-elementary abelian of rank $2\left\lfloor\log _{2}(c)\right\rfloor+2\left\lfloor\log _{2} \frac{c}{3}\right\rfloor+5$, for $c \geq 4$.

- $M_{c}(\Delta)$ has the form $\mathbb{Z}^{2} \times \mathcal{A}_{c}$, where $\mathcal{A}_{c}$ is an abelian 2-group of rank $\left\lfloor\log _{2} \frac{c}{3}\right\rfloor$ and exponent $2^{2\left\lfloor\frac{c-6}{2}\right\rfloor+2}$, for $c \geq 6$.

- $M_{c}(\mathrm{BSV})$ has the form $\mathbb{Z}^{2} \times \mathcal{B}_{c}$, where $\mathcal{B}_{c}$ is an abelian 2-group of rank $\left\lfloor\log _{2} \frac{c}{5}\right\rfloor+$ $\left\lfloor\log _{2} \frac{c}{9}\right\rfloor+3$ and exponent $2^{2\left\lfloor\frac{c-4}{2}\right\rfloor+1}$, for $c \geq 4$.

- For a prime power $d$, the group $M_{c}\left(\Gamma_{d}\right)$ has exponent d for c large enough; its rank is an increasing function in $c$ which exhibits a periodic pattern.

In particular, all of these groups have an infinitely generated Schur multiplier and are therefore not finitely presentable.

Further details on the periodicities and the computational evidence for them are given in Section E.6.

\section{E.2 Preliminaries}

In the following, we recall the basic notion of invariant and finite $L$-presentations and the basic theory of the Schur multiplier of a group. Let $F$ be a finitely generated free group over the alphabet $\mathcal{X}$. Further suppose that $\mathcal{Q}, \mathcal{R} \subset F$ are finite subsets of the free 
group $F$ and $\Phi \subset \operatorname{End}(F)$ is a finite set of endomorphisms of $F$. Then the quadruple $\langle\mathcal{X}|\mathcal{Q}| \Phi \mid \mathcal{R}\rangle$ is a finite L-presentation. It defines the finitely L-presented group

$$
G=\left\langle\mathcal{X} \mid \mathcal{Q} \cup \bigcup_{\varphi \in \Phi^{*}} \mathcal{R}^{\varphi}\right\rangle
$$

where $\Phi^{*}$ denotes the free monoid generated by $\Phi$; that is, the closure of $\Phi \cup\{$ id $\}$ under composition. A finite $L$-presentation $\langle\mathcal{X}|\mathcal{Q}| \Phi \mid \mathcal{R}\rangle$ is invariant if every endomorphism $\varphi \in \Phi$ induces an endomorphism of $G$; that is, if the normal closure of $\mathcal{Q} \cup \bigcup_{\varphi \in \Phi^{*}} \mathcal{R}^{\varphi}$ in $F$ is $\varphi$-invariant. For example, every finite $L$-presentation of the form $\langle\mathcal{X}|\emptyset| \Phi|$ $\mathcal{R}\rangle$ is invariant. Clearly, invariant finite $L$-presentations generalize finite presentations since every finitely presented group $\langle\mathcal{X} \mid \mathcal{R}\rangle$ is finitely $L$-presented by $\langle\mathcal{X}|\emptyset|\{$ id $\}|$ $\mathcal{R}\rangle$. Further examples of invariantly $L$-presented groups are several self-similar groups including the Grigorchuk group [53], the Basilica group [60,61], and the Brunner-SidkiVieira group [29].

In the remainder of this section, we recall the basic theory of the Schur multiplier of a group $G$. Recall that, in general, the Schur multiplier of a finitely presented group is not computable; see [52]. But, for instance, if $G$ is finite, then $M(G)$ can be deduced from a finite presentation of $G$ with the Hopf formula and the Reidemeister-Schreier algorithm. A more effective algorithm for finite permutation groups is described in 82]. Recently, Eick and Nickel [44] described an algorithm for computing the Schur multiplier of a polycyclic group given by a polycyclic presentation.

Let $F$ be a free group and $R$ be a normal subgroup of $F$ so that $G \cong F / R$. Then the Hopf formula gives

$$
M(G) \cong\left(R \cap F^{\prime}\right) /[R, F]
$$

Suppose that $N$ is a normal subgroup of $G$ and let $S$ be a normal subgroup of $F$ so that $S R / R$ corresponds to $N$. Then Blackburn and Evens [24] determined the exact sequence

$$
1 \rightarrow(R \cap[S, F]) /([R, F] \cap[S, F]) \rightarrow M(G) \rightarrow M(G / N) \rightarrow\left(N \cap G^{\prime}\right) /[N, G] \rightarrow 1 .
$$

Applying this sequence to the lower central series term $N=\gamma_{c} G$ yields the exact sequence

$$
1 \rightarrow\left(R \cap \gamma_{c+1} F\right) /\left([R, F] \cap \gamma_{c+1} F\right) \rightarrow M(G) \stackrel{\varphi_{c}}{\rightarrow} M\left(G / \gamma_{c} G\right) \rightarrow \gamma_{c} G / \gamma_{c+1} G \rightarrow 1 .
$$

This gives a filtration $M(G) \geq \operatorname{ker} \varphi_{1} \geq \operatorname{ker} \varphi_{2} \geq \ldots$, called the Dwyer-filtration, of the Schur multiplier of $G$. Note that, if $G$ has a maximal nilpotent quotient of class $c$, then

$$
\bigcap_{c \in \mathbb{N}_{0}} \operatorname{ker} \varphi_{c} \cong\left(R \cap \gamma_{c+1} F\right)[R, F] /[R, F]
$$

However, even if the group $G$ is residually nilpotent, the group $F /[R, F]$ is not necessarily residually nilpotent; see [98] and [25]. Thus the Dwyer-kernel $\bigcap_{c \in \mathbb{N}} \operatorname{ker} \varphi_{c}$ is possibly non-trivial.

We note that the Schur multiplier $M\left(G / \gamma_{c} G\right)$ can be computed with the algorithm in [44] while the isomorphism type of $\gamma_{c} G / \gamma_{c+1} G$ can be computed with the nilpotent 
quotient algorithm in [9]. Therefore, the sequence $M(G) \rightarrow M\left(G / \gamma_{c} G\right) \rightarrow \gamma_{c} G / \gamma_{c+1} G \rightarrow$ 1 allows to determine the size of $M_{c}(G)$ provided that $M\left(G / \gamma_{c} G\right)$ is finite. However, the algorithm described here determines the structure of $M_{c}(G)$ even if the Schur multiplier $M\left(G / \gamma_{c} G\right)$ is infinite.

\section{E.3 Adjusting an Invariant $L$-Presentation}

In order to prove the following theorem, we explicitly describe an algorithm for modifying an invariant $L$-presentation. The resulting $L$-presentation enables us to read off a generating set for the Schur multiplier in Section E.4 Our algorithm generalizes the explicit computations in [56].

Theorem 3.1 Let $\langle\mathcal{X}|\mathcal{Q}| \Phi \mid \mathcal{R}\rangle$ be an invariant finite L-presentation which defines the group $G=F / R$. Then $G$ admits an invariant finite L-presentation $\left\langle\mathcal{X}\left|\mathcal{Q}^{\prime} \cup \mathcal{B}\right| \Phi \mid \mathcal{R}^{\prime}\right\rangle$ with $\mathcal{Q}^{\prime}, \mathcal{R}^{\prime} \subset F^{\prime}$ and $\mathcal{B} \subset F$ satisfying $|\mathcal{B}|=|\mathcal{X}|-\mathfrak{h}\left(G / G^{\prime}\right)$, where $\mathfrak{h}\left(G / G^{\prime}\right)$ denotes the torsion-free rank of $G / G^{\prime}$.

Proof. Since $\langle\mathcal{X}|\mathcal{Q}| \Phi \mid \mathcal{R}\rangle$ is an invariant $L$-presentation, every endomorphism $\varphi \in \Phi$ induces an endomorphism of the group $G$. Thus we have $R^{\varphi} \subseteq R$, for every $\varphi \in \Phi^{*}$. In particular, every image of a relator in $\mathcal{Q} \cup \mathcal{R}$ is a consequence; that is, $\mathcal{Q}^{\varphi} \subset R$ and $\mathcal{R}^{\varphi} \subset R$, for every $\varphi \in \Phi^{*}$.

Write $n=\operatorname{rk}(F)$. Then the abelianization $\pi: F \rightarrow \mathbb{Z}^{n}$ maps every $x \in F$ to its corresponding exponent vector $a_{x} \in \mathbb{Z}^{n}$. Clearly, $\operatorname{ker} \pi=F^{\prime}$ and, since $F^{\prime}$ is fullyinvariant, every $\varphi \in \Phi$ induces an endomorphism of the free abelian group $\mathbb{Z}^{n}$. Therefore, the exponent vector of $x^{\varphi}$ is the image $a_{x} M_{\varphi}$ for some matrix $M_{\varphi} \in \mathbb{Z}^{n \times n}$. Now, the normal subgroup $R F^{\prime}$ maps onto

$$
U=\left\langle a_{q}, a_{r} M_{\varphi} \mid q \in \mathcal{Q}, r \in \mathcal{R}, \varphi \in \Phi^{*}\right\rangle \leq \mathbb{Z}^{n}
$$

As every subgroup of $\mathbb{Z}^{n}$ is generated by at most $n$ elements, the subgroup $U$ is finitely generated. In the following, we may use the spinning algorithm from [9] and Hermite normal form computations to compute a basis for the subgroup $U$ while modifying the $L$-presentation simultaneously.

Let $B$ be a basis of $\left\langle a_{q} \mid q \in \mathcal{Q}\right\rangle$. Then every element $u \in B$ is a $\mathbb{Z}$-linear combination of elements in $\left\{a_{q} \mid q \in \mathcal{Q}\right\}$. Hence, for every $u \in B$, there exists a word $r_{u}$ in the relators in $\mathcal{Q}$ such that $a_{r_{u}}=u$. Define $\mathcal{B}=\left\{r_{u} \mid u \in B\right\}$. Then, for every $q \in \mathcal{Q}$, it holds that $a_{q} \in\langle B\rangle$ as $B$ is a basis and hence, there exists a word $w_{q}$ in the $r_{u}$ 's so that $a_{w_{q}}=a_{q}$. Define $\mathcal{Q}^{\prime}=\left\{q w_{q}^{-1} \mid q \in \mathcal{Q}\right\}$. Then the exponent vector of each element in $\mathcal{Q}^{\prime}$ vanishes and hence $\mathcal{Q}^{\prime} \subset F^{\prime}$. Moreover, the invariant and finite $L$-presentation

$$
\left\langle\mathcal{X}\left|\mathcal{Q}^{\prime} \cup \mathcal{B}\right| \Phi \mid \mathcal{R}\right\rangle
$$

still defines the group $G$ as we only applied Tietze transformations to the given $L$ presentation. 
It remains to force the elements of $\mathcal{R}$ into the derived subgroup $F^{\prime}$. For this purpose, we will use the spinning algorithm from [9] as follows: Initialize $\mathcal{R}^{\prime}=\emptyset$. As long as $\mathcal{R}$ is non-empty, we take an element $r \in \mathcal{R}$ and remove it from $\mathcal{R}$. Then either $a_{r} \in\langle B\rangle$ or $a_{r} \notin\langle B\rangle$ holds. If $a_{r} \in\langle B\rangle$, then there exists a word $w_{r}$ in the $r_{u}$ 's such that $a_{w_{r}}=a_{r}$ and hence, $r w_{r}^{-1} \in F^{\prime}$. In this case we just add $r w_{r}^{-1}$ to $\mathcal{R}^{\prime}$. Note that, for every $\varphi \in \Phi^{*}$, the word $\left(w_{r}^{-1}\right)^{\varphi}$ is a consequence and hence, we can replace the relator $r^{\varphi}$ in the $L$-presentation by $\left(r w_{r}^{-1}\right)^{\varphi}$. The invariant and finite $L$-presentation

$$
\left\langle\mathcal{X}\left|\mathcal{Q}^{\prime} \cup \mathcal{B}\right| \Phi \mid \mathcal{R}^{\prime} \cup \mathcal{R}\right\rangle
$$

still defines the group $G$.

If, on the other hand, $a_{r} \notin\langle B\rangle$ holds, we enlarge the current basis $B$ and modify the set $\mathcal{B}$. Let $B^{\prime}$ be a basis for $\left\langle B \cup\left\{a_{r}\right\}\right\rangle$. Then every $v \in B^{\prime}$ is a $\mathbb{Z}$-linear combination of the elements in $B \cup\left\{a_{r}\right\}$ and hence, there exists a word $\tilde{r}_{v}$ in $\mathcal{B} \cup\{r\}$ such that $a_{\tilde{r}_{v}}=v$. Define $\mathcal{B}=\left\{\tilde{r}_{v} \mid v \in B^{\prime}\right\}$. Then, by construction, either $|\mathcal{B}|=|B|+1$ or $|\mathcal{B}|=|B|$ holds. In the latter case, there is an element $u \in B$ so that $u \in\left\langle(B \backslash\{u\}) \cup\left\{a_{r}\right\}\right\rangle$ holds. Thus, there exists a word $w_{u}$ in the elements of $\mathcal{B}$ such that $a_{w_{u}}=u$ and hence, $r_{u} w_{u}^{-1} \in F^{\prime}$. In this case, we add $r_{u} w_{u}^{-1}$ to $\mathcal{Q}^{\prime}$ and add the images $\left\{r_{u}^{\varphi} \mid \varphi \in \Phi\right\}$ to $\mathcal{R}$. This yields an invariant and finite $L$-presentation $\left\langle\mathcal{X}\left|\mathcal{Q}^{\prime} \cup \mathcal{B}\right| \Phi \mid \mathcal{R}^{\prime} \cup \mathcal{R}\right\rangle$, with $\mathcal{Q}^{\prime}, \mathcal{R}^{\prime} \subset F^{\prime}$, which still defines the group $G$.

As ascending chains of subgroups in $\mathbb{Z}^{n}$ terminate, eventually every exponent vector of an element in $\mathcal{R}$ is contained in the subgroup $\langle B\rangle$ and hence, the algorithm described above eventually terminates. Clearly, the basis $B$ is then a basis for the subgroup $U$ in (E.2). As shown in [9], the abelian quotient $G / G^{\prime}$ is isomorphic to the factor $\mathbb{Z}^{n} / U$. Its torsion-free rank is $n-|B|$ as claimed above.

In the following example, we recall the explicit computations in [56] for the Grigorchuk group $\mathfrak{G}$.

Example 3.2 Consider the Grigorchuk group $\mathfrak{G}$ with its invariant L-presentation

$$
\left.\mathfrak{G} \cong\left\langle\{a, b, c, d\}\left|\left\{a^{2}, b^{2}, c^{2}, d^{2}, b c d\right\}\right|\{\sigma\}\right|\left\{(a d)^{4},(\text { adacac })^{4}\right\}\right\rangle
$$

where $\sigma$ is the free group endomorphism induced by the mapping

$$
\sigma:\left\{\begin{array}{rll}
a & \mapsto c & c^{a} \\
b & \mapsto & d \\
c & \mapsto b \\
d & \mapsto c .
\end{array}\right.
$$

As the exponent vectors $(2,0,0,0),(0,1,1,1),(0,0,2,0)$, and $(0,0,0,2)$ of the relations $a^{2}, b c d, c^{2}$, and $d^{2}$, respectively, are $\mathbb{Z}$-linearly independent forming a basis for the subgroup $U$ in (E.2), we can modify this presentation so that the relations become

$$
a^{2}, c^{2}, d^{2}, b c d, b^{2}(b c d)^{-2} c^{2} d^{2}, \sigma^{k}\left((a d)^{4} a^{-4} d^{-4}\right), \sigma^{k}\left((a d a c a c)^{4} a^{-12} c^{-8} d^{-4}\right),
$$


for every $k \in \mathbb{N}_{0}$. Since the L-presentation is invariant, the images $\sigma^{k}\left(a^{-4} d^{-4}\right)$ and $\sigma^{k}\left(a^{-12} c^{-8} d^{-4}\right)$ are consequences. Hence, the invariant finite L-presentation

$$
\left\langle\{a, b, c, d\}\left|\left\{b^{2}(b c d)^{-2} c^{2} d^{2}\right\} \cup\left\{a^{2}, c^{2}, d^{2}, b c d\right\}\right|\{\sigma\} \mid \mathcal{R}^{\prime}\right\rangle,
$$

where $\mathcal{R}^{\prime}=\left\{(a d)^{4} a^{-4} d^{-4},(\text { adacac })^{4} a^{-12} c^{-8} d^{-4}\right\}$, defines the Grigorchuk group $\mathfrak{G}$ and, as $\mathfrak{G} / \mathfrak{G}^{\prime} \cong \mathbb{Z}_{2}^{3}$, it has the form as claimed in Theorem 3.1.

\section{E.4 A Generating Set for the Schur Multiplier}

Let $G$ be a finitely generated group. We will use the results of Theorem 3.1 and the Hopf formula to give a generating set for the Schur multiplier of $G$ if $G$ is invariantly finitely $L$-presented. Suppose that $F$ is a finitely generated free group and $R$ is a normal subgroup of $F$ so that $G \cong F / R$. Then $F /[R, F]$ is a central extension of $R /[R, F]$ by the group $G$ and the subgroup $R /[R, F]$ contains $\left(R \cap F^{\prime}\right) /[R, F]$. By the Hopf formula, the latter subgroup is isomorphic to the Schur multiplier of $G$. Further, the subgroup $R /[R, F]$ decomposes as follows.

Proposition 4.3 Let $G \cong F / R$ with a finitely generated free group $F$. Then we have that

$$
R /[R, F] \cong \mathbb{Z}^{\mathrm{rk}(F)-\mathfrak{h}\left(G / G^{\prime}\right)} \oplus M(G) .
$$

Proof. The factor $R F^{\prime} / F^{\prime}$ is free abelian with torsion-free $\operatorname{rank} \operatorname{rk}(F)-\mathfrak{h}\left(G / G^{\prime}\right)$. Since $R F^{\prime} / F^{\prime} \cong R /\left(R \cap F^{\prime}\right)$ is free abelian, the subgroup $\left(R \cap F^{\prime}\right) /[R, F]$ has a free abelian complement of $\operatorname{rank} \operatorname{rk}(F)-\mathfrak{h}\left(G / G^{\prime}\right)$ and thus, the central subgroup $R /[R, F]$ decomposes as claimed above.

As $R /[R, F]$ is central in $F /[R, F]$, it is generated by the images of the normal generators of $R$. Thus, in particular, if $R$ is finitely generated as normal subgroup (that is, if $G$ is finitely presentable), then $R /[R, F]$ is a finitely generated abelian group and so is its subgroup $\left(R \cap F^{\prime}\right) /[R, F]$.

If $G$ is finite, then $R /[R, F]$ is an abelian subgroup with finite index in $F /[R, F]$. A finite presentation for $F /[R, F]$ can be obtained from a finite presentation of $G$. Then the Reidemeister-Schreier algorithm yields a finite presentation for $R /[R, F]$ from which the isomorphism type of $M(G)$ is obtained easily.

If $G$ is polycyclic, then it is finitely presentable and hence, the group $F /[R, F]$ is an extension of a finitely generated abelian group by a polycyclic group. In particular, $F /[R, F]$ is polycyclic in this case. A consistent polycyclic presentation for $F /[R, F]$ can be computed with the algorithm in [44. This polycyclic presentation enables us to read off the isomorphism type of $R /[R, F]$ and, by Proposition 4.3, the isomorphism type of $M(G)$. If $G$ is finitely generated and nilpotent of class $c$, then $F /[R, F]$ is nilpotent of class at most $c+1$. If $G$ is given by a weighted nilpotent presentation, then the algorithm in [107] computes a weighted nilpotent presentation for $F /[R, F]$.

We now consider the case of an invariantly finitely $L$-presented group $G$. Even though its Schur multiplier is not computable in general, the following theorem yields a generating set for $M(G)$ as subgroup of $R /[R, F]$. 
Theorem 4.4 Let $\left\langle\mathcal{X}\left|\mathcal{Q}^{\prime} \cup \mathcal{B}\right| \Phi \mid \mathcal{R}^{\prime}\right\rangle$ be an invariant finite L-presentation of $G$ as provided by Theorem [3.1. Further let $\pi: F \rightarrow F /[R, F], x \mapsto \bar{x}$ denote the natural homomorphism. Then we have that

$$
M(G) \cong\left\langle\bar{q}, \overline{r^{\varphi}} \mid q \in \mathcal{Q}^{\prime}, r \in \mathcal{R}^{\prime}, \varphi \in \Phi^{*}\right\rangle .
$$

Proof. Clearly, $R /[R, F]$ is generated by the images of $\mathcal{Q}^{\prime} \cup \mathcal{B} \cup \bigcup_{\varphi \in \Phi^{*}}\left(\mathcal{R}^{\prime}\right)^{\varphi}$. As the relators in $\mathcal{Q}^{\prime} \cup \mathcal{R}^{\prime}$ are contained in $F^{\prime}$, it holds that

$$
\left\{\bar{q}, \overline{r^{\varphi}} \mid q \in \mathcal{Q}^{\prime}, r \in \mathcal{R}^{\prime}, \varphi \in \Phi^{*}\right\} \subseteq\left(R \cap F^{\prime}\right) /[R, F] .
$$

We are left with the relators in $\mathcal{B}$. Recall that we have $|\mathcal{B}|=\operatorname{rk}(F)-\mathfrak{h}\left(G / G^{\prime}\right)$. Hence, the images $\{\bar{r} \mid r \in \mathcal{B}\}$ generate a free abelian complement to the Schur multiplier $\left(R \cap F^{\prime}\right) /[R, F]$ in $R /[R, F]$. Therefore, the images in (E.4) necessarily generate $(R \cap$ $\left.F^{\prime}\right) /[R, F]$.

As the group $G$ in Theorem 4.4 is invariantly $L$-presented, for every endomorphism $\varphi \in \Phi$, we have $R^{\varphi} \subseteq R$ and $[R, F]^{\varphi} \subseteq[R, F]$. Therefore, every $\varphi \in \Phi$ also induces an endomorphism of $F /[R, F]$ which fixes the subgroup $R /[R, F]$. Further, as $F^{\prime}$ is fullyinvariant, every such $\varphi$ induces an endomorphism $\bar{\varphi}$ of $\left(R \cap F^{\prime}\right) /[R, F]$. This yields that

$$
M(G) \cong\left\langle\bar{q}, \bar{r}^{\bar{\varphi}} \mid q \in \mathcal{Q}^{\prime}, r \in \mathcal{R}^{\prime}, \varphi \in \Phi^{*}\right\rangle
$$

and hence, the free monoid $\Phi^{*}$ induces a $\Phi^{*}$-module structure on the Schur multiplier $M(G)$ in a natural way:

Lemma 4.5 Let $\langle\mathcal{X}|\mathcal{Q}| \Phi \mid \mathcal{R}\rangle$ be an invariant finite L-presentation. Then the Schur multiplier $M(G)$ is finitely generated as a $\Phi^{*}$-module.

In particular, the Schur multiplier $M(G)$ has the form $A \oplus \bigoplus_{\Phi^{*}} B$ with finitely generated abelian groups $A$ and $B$; see [6].

We proceed with Example 3.2 by describing a generating set for the Schur multiplier of the Grigorchuk group as provided by Theorem 4.4, cf. [56].

Example 4.6 Consider the invariant finite L-presentation of the Grigorchuk group $\mathfrak{G}$ as determined in Example 3.2. Then the images of

$$
b^{2}(b c d)^{-2} c^{2} d^{2}, \sigma^{k}\left((a d)^{4} a^{-4} d^{-4}\right), \sigma^{k}\left((\text { adacac })^{4} a^{-12} c^{-8} d^{-4}\right), \quad \text { with } k \in \mathbb{N}_{0},
$$

in $F /[R, F]$, generate the subgroup $\left(R \cap F^{\prime}\right) /[R, F]$. The images in $F /[R, F]$ of the relations $a^{2}, c^{2}, d^{2}$, and bcd generate a free abelian complement to the Schur multiplier $\left(R \cap F^{\prime}\right) /[R, F]$ in $R /[R, F]$.

\section{E.5 Approximating the Schur Multiplier}

We finally describe our algorithm for approximating the Schur multiplier of an invariantly finitely $L$-presented group $G$. Let $\langle\mathcal{X}|\mathcal{Q}| \Phi \mid \mathcal{R}\rangle$ be an invariant finite $L$-presentation 
defining the group $F / R$ so that $G \cong F / R$. Then $G$ is finitely generated and hence, its lower central series quotient $G / \gamma_{c} G$ is polycyclic. The nilpotent quotient algorithm in [9] computes a weighted nilpotent presentation for $G / \gamma_{c} G$ together with the natural homomorphism $\pi: F \rightarrow G / \gamma_{c} G$. In [107], Nickel described a covering-algorithm which, given a weighted nilpotent presentation for $G / \gamma_{c} G$ and the homomorphism $\pi$, computes a polycyclic presentation for $F /\left[R \gamma_{c} F, F\right]$ together with the natural homomorphism $\bar{\pi}: F \rightarrow$ $F /\left[R \gamma_{c} F, F\right]$. The homomorphism $\bar{\pi}$ induces the homomorphism $\varphi_{c}: M(G) \rightarrow M\left(G / \gamma_{c} G\right)$ as follows: By Theorem [3.1, the group $G$ has an invariant finite $L$-presentation of the form

$$
\left\langle\mathcal{X}\left|\mathcal{Q}^{\prime} \cup \mathcal{B}\right| \Phi \mid \mathcal{R}^{\prime}\right\rangle, \quad \text { with } \mathcal{Q}^{\prime}, \mathcal{R}^{\prime} \subset F^{\prime}
$$

and $|\mathcal{B}|=|\mathcal{X}|-\mathfrak{h}\left(G / G^{\prime}\right)$. Now, by Theorem 4.4, the images of $\mathcal{Q}^{\prime} \cup \bigcup_{\varphi \in \Phi^{*}}\left(\mathcal{R}^{\prime}\right)^{\varphi}$ in $F /[R, F]$ generate the subgroup $\left(R \cap F^{\prime}\right) /[R, F]$. Similarly, the their images in $F /\left[R \gamma_{c} F, F\right]$ generate the subgroup $\left(R \cap F^{\prime}\right)\left[R \gamma_{c} F, F\right] /\left[R \gamma_{c} F, F\right]$. Since $\left[R \gamma_{c} F, F\right]=$ $[R, F] \gamma_{c+1} F$, we have that

$$
\left(R \cap F^{\prime}\right)\left[R \gamma_{c} F, F\right] /\left[R \gamma_{c} F, F\right]=\left(R \gamma_{c+1} F \cap F^{\prime}\right) /\left[R \gamma_{c} F, F\right] .
$$

The latter subgroup is contained in $\left(R \gamma_{c} F \cap F^{\prime}\right) /\left[R \gamma_{c} F, F\right]$ which is isomorphic to the Schur multiplier $M\left(G / \gamma_{c} G\right)$.

As the group $G$ is invariantly $L$-presented, every $\varphi \in \Phi$ induces an endomorphism $\tilde{\varphi}$ of $R \gamma_{c} F /\left[R \gamma_{c} F, F\right]$. This yields, that the image of $M(G)$ in $M\left(G / \gamma_{c} G\right)$ has the form

$$
\left\langle q^{\bar{p}},\left(r^{\bar{\pi}}\right)^{\tilde{\varphi}} \mid q \in \mathcal{Q}^{\prime}, r \in \mathcal{R}^{\prime}, \varphi \in \Phi^{*}\right\rangle .
$$

This can be used to investigate the $\Phi^{*}$-module structure of $M(G)$ by considering the finitely generated Dwyer quotients $M_{c}(G)$. In our algorithm, we use Hermite normal form computations in a spinning algorithm for computing a finite generating set of the subgroup in (E.6). We summarize our algorithm as follows: Write $G=F / R$.

DwyerQuotient $(G, c)$

Compute an invariant finite $L$-presentation as in Theorem 3.1 .

Compute a weighted nilpotent presentation for $G / \gamma_{c} G$ together with the natural homomorphism $F \rightarrow G / \gamma_{c} G$.

Compute a polycyclic presentation for the group $F /\left[R \gamma_{c} F, F\right]$ together with the natural homomorphism $F \rightarrow F /\left[R \gamma_{c} F, F\right]$.

Translate each $\varphi \in \Phi$ to an endomorphism of the group $F /\left[R \gamma_{c} F, F\right]$ and restrict this endomorphism to $\left(R \gamma_{c+1} F \cap F^{\prime}\right) /\left[R \gamma_{c} F, F\right]$.

Use the spinning algorithm to compute a finite generating set for the image $\left(R \gamma_{c+1} F \cap F^{\prime}\right) /\left[R \gamma_{c} F, F\right]$.

\section{E.6 Applications}

The algorithm described in the first part is available in the NQL-package [65] of the computer algebra system GAP; see [50]. We parallelized the algorithm in [9] to enlarge 
the possible depths in the lower central series reached in this section. We show the successful application of our algorithm to the following invariantly finitely $L$-presented testbed groups studied in [6] and [9]:

- The Grigorchuk group $\mathfrak{G}$, see [53], with its invariant finite $L$-presentation from [96]; see also [56] and Example 3.2.

- the twisted twin $\overline{\mathfrak{G}}$ of the Grigorchuk group, see [14], with its invariant finite $L$ presentation from [14];

- the Grigorchuk super-group $\tilde{\mathfrak{G}}$, see [11, with its invariant finite $L$-presentation from [6];

- the Basilica group $\Delta$, see [60,61], with its invariant finite $L$-presentation from [16]; and

- the Brunner-Sidki-Vieira group BSV, see [29], with its invariant finite $L$-presentation from [6].

In Section E.6, we further applied our algorithm to several generalized FabrykowskiGupta groups: an infinite family of finitely $L$-presented groups $\Gamma_{p}$ introduced in [57]. Invariant finite $L$-presentations for these groups were computed in [9].

\section{Aspects of the Implementation of our Algorithm in GAP}

Table E.1 shows some performance data of the implementation of our algorithm in the NQL-package of the computer-algebra-system GAP. All timings displayed below have been obtained on an Intel Pentium Core 2 Quad with clock speed $2.83 \mathrm{GHz}$ using a single core. We applied our algorithm with a time limit of two hours. Then the computations have been stopped and the total time used to compute a weighted nilpotent presentation for the quotient $G / \gamma_{c} G$ and the total time to compute the Dwyer quotient $M_{c}(G)$ have been listed. Every application completed within 1 GB of memory.

Table E.1: Performance data of our implementation in GAP

\begin{tabular}{cccc}
\hline \multirow{2}{*}{$G$} & $c$ & \multicolumn{2}{c}{ Time (h:min) for } \\
& & $G / \gamma_{c+1} G$ & $M_{c+1}(G)$ \\
\hline $\mathfrak{G}$ & 90 & $1: 47$ & $0: 07$ \\
$\overline{\mathfrak{G}}$ & 54 & $1: 44$ & $0: 09$ \\
$\tilde{\mathfrak{G}}$ & 44 & $1: 32$ & $0: 13$ \\
$\Delta$ & 42 & $1: 31$ & $0: 16$ \\
$\mathrm{BSV}$ & 35 & $1: 10$ & $0: 21$ \\
$\Gamma_{3}$ & 75 & $1: 46$ & $0: 04$ \\
\hline
\end{tabular}

\begin{tabular}{cccc}
\hline \multirow{3}{*}{$G$} & $c$ & \multicolumn{2}{c}{ Time (h:min) for } \\
& & $G / \gamma_{c+1} G$ & $M_{c+1}(G)$ \\
\hline$\Gamma_{4}$ & 71 & $1: 50$ & $0: 07$ \\
$\Gamma_{5}$ & 55 & $1: 40$ & $0: 04$ \\
$\Gamma_{7}$ & 46 & $1: 40$ & $0: 03$ \\
$\Gamma_{8}$ & 56 & $1: 54$ & $0: 06$ \\
$\Gamma_{9}$ & 61 & $1: 44$ & $0: 06$ \\
$\Gamma_{11}$ & 35 & $1: 54$ & $0: 02$ \\
\hline
\end{tabular}

We note that for the results shown in the remainder of this section we used a parallel version of the algorithm for computing $G / \gamma_{c+1} G$. 


\section{On the Dwyer Quotients of the Testbed-Groups}

The Dwyer quotient $M_{c}(G)=M(G) / \operatorname{ker} \varphi_{c}$ is a finitely generated abelian group and hence, it can be described by its abelian invariants or, if the group is $p$-elementary abelian, by its $p$-rank. Here the list $\left(c_{1}, \ldots, c_{n}\right)$ stands for the group $\mathbb{Z}_{c_{1}} \oplus \cdots \oplus \mathbb{Z}_{c_{n}}$. For abbreviation, we will write $a^{[\ell]}$ if the term $a$ occurs in $\ell$ consecutive places in a list. In the following we summarize our computational results for the testbed groups.

The Grigorchuk group $\mathfrak{G}$ was shown in 53 to be an explicit counter-example to the general Burnside problem: it is a finitely generated infinite 2-torsion group. Furthermore, the Grigorchuk group is a first example of a group with an intermediate word-growth. In [96], Lysënok determined a first $L$-presentation for the group $\mathfrak{G}$; see Example 3.2 . Even though it was already proposed in $[53$ that the Grigorchuk group $\mathfrak{G}$ is not finitely presentable, a proof was not derived until [56] where Grigorchuk explicitly computed the Schur multiplier of $\mathfrak{G}$ : it is infinitely generated 2-elementary abelian. We have computed the Dwyer quotients $M_{c}(\mathfrak{G})$, for $1 \leq c \leq 301$. These quotients are 2-elementary abelian with the following 2-ranks

$$
1,2,3^{[3]}, 5^{[6]}, 7^{[12]}, 9^{[24]}, 11^{[48]}, 13^{[96]}, 15^{[110]} .
$$

This suggests the following conjecture.

Conjecture A The Grigorchuk group $\mathfrak{G}$ satisfies

$$
M_{c}(\mathfrak{G}) \cong\left\{\begin{array}{cl}
\mathbb{Z}_{2} \text { or }\left(\mathbb{Z}_{2}\right)^{2}, & \text { if } c=1 \text { or } c=2, \text { respectively } \\
\left(\mathbb{Z}_{2}\right)^{2 m+3}, & \text { if } c \in\left\{3 \cdot 2^{m}, \ldots, 3 \cdot 2^{m+1}-1\right\}
\end{array}\right\},
$$

with $m \in \mathbb{N}_{0}$.

Further experiments suggest that the Schur multiplier of the Grigorchuk group $\mathfrak{G}$ has the $\{\sigma\}^{*}$-module structure, as given by Lemma 4.5, of the form $\mathbb{Z}_{2} \oplus\left(\mathbb{Z}_{2}[\sigma]\right)^{2}$ where $\sigma$ fixes the first component.

The twisted twin $\overline{\mathfrak{G}}$ of the Grigorchuk group was introduced in [14]. It is invariantly finitely $L$-presented by

$$
\left\langle\{a, b, c, d\}\left|\left\{a^{2}, b^{2}, c^{2}, d^{2}\right\}\right|\{\bar{\sigma}\} \mid\left\{\left[d^{a}, d\right],\left[d, c^{a} b\right],\left[d,\left(c^{a} b\right)^{c}\right],\left[d,\left(c^{a} b\right)^{c}\right],\left[c^{a} b, c b^{a}\right]\right\}\right\rangle
$$

where $\tilde{\sigma}$ is the free group endomorphism induced by the mapping

$$
\tilde{\sigma}:\left\{\begin{array}{rlr}
a & \mapsto c^{a} \\
b & \mapsto d \\
c & \mapsto b^{a} \\
d & \mapsto c .
\end{array}\right.
$$

We have computed the Dwyer quotients $M_{c}(\overline{\mathfrak{G}})$, for $1 \leq c \leq 144$. These quotients are 2-elementary abelian with the following 2-ranks

$$
2,5,7,8^{[2]}, 11^{[2]}, 12^{[4]}, 15^{[4]}, 16^{[8]}, 19^{[8]}, 20^{[16]}, 23^{[16]}, 24^{[32]}, 27^{[32]}, 28^{[17]} \text {. }
$$

This suggests the following conjecture. 
Conjecture B The twisted twin $\overline{\mathfrak{G}}$ of the Grigorchuk group satisfies

$$
M_{c}(\overline{\mathfrak{G}}) \cong\left\{\begin{array}{cl}
\left(\mathbb{Z}_{2}\right)^{2},\left(\mathbb{Z}_{2}\right)^{5}, \text { or }\left(\mathbb{Z}_{2}\right)^{7}, & \text { if } c=1, c=2, \text { or } c=3, \text { resp. } \\
\left(\mathbb{Z}_{2}\right)^{4(m+1)+4}, & \text { if } c \in\left\{2^{m+2}, \ldots, 2^{m+2}+2^{m+1}-1\right\} \\
\left(\mathbb{Z}_{2}\right)^{4(m+1)+7}, & \text { if } c \in\left\{2^{m+2}+2^{m+1}, \ldots, 2^{m+3}-1\right\}
\end{array}\right\},
$$

with $m \in \mathbb{N}_{0}$.

Further experiments suggest that the Schur multiplier of $\overline{\mathfrak{G}}$ has the $\{\bar{\sigma}\}^{*}$-module structure, as given by Lemma 4.5 , of the form $\left(\mathbb{Z}_{2}[\bar{\sigma}]\right)^{4}$; for a proof see [14].

The Grigorchuk super-group $\tilde{\mathfrak{G}}$ was introduced in [11]. It contains the Grigorchuk group $\mathfrak{G}$ as an infinite-index subgroup and it is another example of a group with an intermediate word-growth. In [6], it was shown that $\tilde{\mathfrak{G}}$ admits the invariant finite $L$ presentation $\langle\{\tilde{a}, \tilde{b}, \tilde{c}, \tilde{d}\}|\emptyset|\{\tilde{\sigma}\} \mid \mathcal{R}\rangle$ where

$$
\mathcal{R}=\left\{a^{2},[\tilde{b}, \tilde{c}],\left[\tilde{c}, \tilde{c}^{a}\right],\left[\tilde{c}, \tilde{d}^{a}\right],\left[\tilde{d}, \tilde{d}^{a}\right],\left[\tilde{c}^{a \tilde{b}},\left(\tilde{c}^{a \tilde{b}}\right)^{a}\right],\left[\tilde{c}^{a \tilde{b}},\left(\tilde{d}^{a \tilde{b}}\right)^{a}\right],\left[\tilde{d}^{a \tilde{b}},\left(\tilde{d}^{a \tilde{b}}\right)^{a}\right]\right\}
$$

and $\tilde{\sigma}$ is the free group endomorphism induced by the mapping

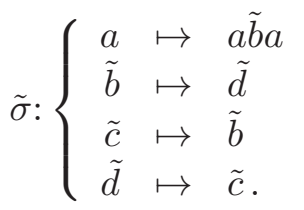

The Schur multiplier of the group $\tilde{\mathfrak{G}}$ is still unknown. We have computed the Dwyer quotients $M_{c}(\tilde{\mathfrak{G}})$, for $1 \leq c \leq 232$. These quotients are 2-elementary abelian with the following 2-ranks

$$
3,6,7,9^{[2]}, 11^{[2]}, 13^{[4]}, 15^{[4]}, 17^{[8]}, 19^{[8]}, 21^{[16]}, 23^{[16]}, 25^{[32]}, 27^{[32]}, 29^{[64]}, 31^{[41]} .
$$

This suggests the following conjecture.

Conjecture $\mathbf{C}$ The Grigorchuk super-group $\tilde{\mathfrak{G}}$ satisfies

$$
M_{c}(\tilde{\mathfrak{G}}) \cong\left\{\begin{array}{cl}
\left(\mathbb{Z}_{2}\right)^{3},\left(\mathbb{Z}_{2}\right)^{6}, \text { or }\left(\mathbb{Z}_{2}\right)^{7}, & \text { if } c=1,2, \text { or } 3, \text { respectively } \\
\left(\mathbb{Z}_{2}\right)^{4 m+5}, & \text { if } c \in\left\{2 \cdot 2^{m}, \ldots, 3 \cdot 2^{m}-1\right\} \\
\left(\mathbb{Z}_{2}\right)^{4 m+7}, & \text { if } c \in\left\{3 \cdot 2^{m}, \ldots, 2 \cdot 2^{m+1}-1\right\}
\end{array}\right\},
$$

with $m \in \mathbb{N}$.

Further experiments suggest that the Schur multiplier of the Grigorchuk super-group has the $\{\tilde{\sigma}\}^{*}$-module structure, as given by Lemma 4.5 , of the form $\left(\mathbb{Z}_{2}\right)^{3} \oplus\left(\mathbb{Z}_{2}[\tilde{\sigma}]\right)^{4}$, where $\tilde{\sigma}$ cyclically permutes the first component.

The Basilica group $\Delta$ was introduced in [60,61] as a torsion-free group defined by a three-state automaton. Bartholdi and Virág [16] computed the following invariant finite $L$-presentation:

$$
\Delta \cong\left\langle\{a, b\}|\emptyset|\{\sigma\} \mid\left\{\left[a, a^{b}\right]\right\}\right\rangle
$$


where $\sigma$ is the free group endomorphism induced by the mapping

$$
\sigma:\left\{\begin{array}{rll}
a & \mapsto b^{2} \\
b & \mapsto a
\end{array}\right.
$$

We have computed the Dwyer quotients $M_{c}(\Delta)$, for $1 \leq c \leq 103$. These quotients satisfy the following conjecture.

Conjecture D The Basilica group $\Delta$ satisfies

$$
M_{c}(\Delta) \cong \mathbb{Z}^{2} \oplus \bigoplus_{\ell \in \mathbb{N}} \mathcal{A}_{\ell}(c), \quad \text { for each } c \geq 2,
$$

where the groups $\mathcal{A}_{\ell}(c)$ are given as follows:

$$
\mathcal{A}_{1}(c)=\left\{\begin{array}{cl}
0, & \text { if } c \in\{1, \ldots, 5\} \\
\mathbb{Z}_{2^{2(m+1)}}, & \text { if } c \in\{2 m+6,2 m+7\}
\end{array}\right\}
$$

and

$$
\mathcal{A}_{\ell}(c)=\left\{\begin{array}{cl}
0, & \text { if } c \in\left\{1, \ldots, 3 \cdot 2^{\ell+1}-1\right\} \\
\mathbb{Z}_{2^{2 m+1}}, & \text { if } c \in\left\{(3+m) \cdot 2^{\ell+1}, \ldots,(3+m) \cdot 2^{\ell+1}+2^{\ell-1}-1\right\} \\
\mathbb{Z}_{2^{2 m+2}}, & \text { if } c \in\left\{(3+m) \cdot 2^{\ell+1}+2^{\ell-1}, \ldots,(4+m) \cdot 2^{\ell+1}-1\right\}
\end{array}\right\}
$$

with $m \in \mathbb{N}_{0}$. Hence, the Basilica group $\Delta$ is not finitely presentable.

The Brunner-Sidki-Vieira group BSV was introduced in [29] as a just-non-solvable, torsion-free group acting on the binary tree. The authors also gave the following invariant finite $L$-presentation:

$$
\mathrm{BSV} \cong\left\langle\{a, b\}|\emptyset|\{\varepsilon\} \mid\left\{\left[b, b^{a}\right],\left[b, b^{a^{3}}\right]\right\}\right\rangle
$$

where $\varepsilon$ is the free group endomorphism induced by the mapping

$$
\varepsilon:\left\{\begin{aligned}
a & \mapsto a^{2} \\
b & \mapsto a^{2} b^{-1} a^{2}
\end{aligned}\right.
$$

We have computed the Dwyer quotients $M_{c}(\mathrm{BSV})$, for $1 \leq c \leq 53$. These quotients satisfy the following conjecture.

Conjecture E The Brunner-Sidki-Vieira group BSV satisfies

$$
M_{c}(\mathrm{BSV}) \cong \mathbb{Z}^{2} \oplus \mathcal{A}(c) \oplus \bigoplus_{\ell \in \mathbb{N}} \mathcal{B}_{\ell}(c) \oplus \bigoplus_{\ell \in \mathbb{N}} \mathcal{C}_{\ell}(c), \quad \text { for each } c \geq 2
$$

where the groups $\mathcal{A}(c), \mathcal{B}_{\ell}(c)$, and $\mathcal{C}_{\ell}(c)$ are given as follows:

$$
\mathcal{A}(c)=\left\{\begin{array}{cl}
0, & \text { if } c \in\{1, \ldots, 3\} \\
\mathbb{Z}_{2^{2 m+1}}, & \text { if } c \in\{2 m+4,2 m+5\}
\end{array}\right\}
$$


with $m \in \mathbb{N}_{0}$. Additionally, for each $\ell \in \mathbb{N}$, we have

$$
\mathcal{B}_{\ell}(c)=\left\{\begin{array}{cl}
0, & \text { if } c \in\left\{1, \ldots, 5 \cdot 2^{\ell-1}-1\right\} \\
\mathbb{Z}_{2^{4 m+1}}, & \text { if } c \in\left\{2^{\ell+2} m+5 \cdot 2^{\ell-1}, \ldots, 2^{\ell+2} m+6 \cdot 2^{\ell-1}-1\right\} \\
\mathbb{Z}_{2^{4 m+2}}, & \text { if } c \in\left\{2^{\ell+2} m+6 \cdot 2^{\ell-1}, \ldots, 2^{\ell+2} m+10 \cdot 2^{\ell-1}-1\right\} \\
\mathbb{Z}_{2^{4 m+4}}, & \text { if } c \in\left\{2^{\ell+2} m+10 \cdot 2^{\ell-1}, \ldots, 2^{\ell+2} m+13 \cdot 2^{\ell-1}-1\right\}
\end{array}\right\}
$$

and

$$
\mathcal{C}_{\ell}(c)=\left\{\begin{array}{cl}
0, & \text { if } c \in\left\{1, \ldots, 9 \cdot 2^{\ell-1}-1\right\} \\
\mathbb{Z}_{2^{4 m+1},} & \text { if } c \in\left\{2^{\ell+2} m+9 \cdot 2^{\ell-1}, \ldots, 2^{\ell+2} m+12 \cdot 2^{\ell-1}-1\right\} \\
\mathbb{Z}_{2^{4 m+2}}, & \text { if } c \in\left\{2^{\ell+2} m+12 \cdot 2^{\ell-1}, \ldots, 2^{\ell+2} m+14 \cdot 2^{\ell-1}-1\right\} \\
\mathbb{Z}_{2^{4 m+3},}, & \text { if } c \in\left\{2^{\ell+2} m+14 \cdot 2^{\ell-1}, \ldots, 2^{\ell+2} m+16 \cdot 2^{\ell-1}-1\right\} \\
\mathbb{Z}_{2^{4 m+4}}, & \text { if } c \in\left\{2^{\ell+2} m+16 \cdot 2^{\ell-1}, \ldots, 2^{\ell+2} m+17 \cdot 2^{\ell-1}-1\right\}
\end{array}\right\}
$$

with $m \in \mathbb{N}_{0}$. Hence, the Brunner-Sidki-Vieira group BSV is not finitely presentable.

\section{On the Dwyer Quotients of some Fabrykowski-Gupta Groups}

The Fabrykowski-Gupta group $\Gamma_{3}$ was introduced in [45] as an example of a group with an intermediate word-growth. For every positive integer $d$, Grigorchuk [57] described a generalization $\Gamma_{d}$ of the Fabrykowski-Gupta group $\Gamma_{3}$. A rather longish invariant finite $L$-presentation was computed in [9]. Further, it was shown that, if $d$ is not a prime-power, the group $\Gamma_{d}$ has a maximal nilpotent quotient. This latter quotient is isomorphic to the maximal nilpotent quotient of the wreath product $\mathbb{Z}_{d} \imath \mathbb{Z}_{d}$. We therefore consider only those groups $\Gamma_{d}$ which admit a 'rich' lower central series; that is, the index $d$ is a prime-power.

Let $d \in\{3,5,7,11\}$ be a prime. Then the Dwyer quotients $M_{c}\left(\Gamma_{d}\right)$ are $d$-elementary abelian with the following $d$-ranks.

\begin{tabular}{cc}
\hline$d$ & $\operatorname{rk}\left(M_{c}\left(\Gamma_{d}\right)\right)$ \\
\hline 3 & $0^{[2]}, 1^{[3]}, 2^{[0]}, 3^{[9]}, 4^{[1]}, 5^{[26]}, 6^{[4]}, 7^{[77]}, 8^{[13]}, 9^{[12]}$ \\
5 & $0^{[1]}, 1^{[4]}, 2^{[2]}, 3^{[20]}, 4^{[10]}, 5^{[100]} 6^{[2]}$ \\
7 & $0^{[1]}, 1^{[2]}, 2^{[6]}, 3^{[2]}, 4^{[14]}, 5^{[42]}, 6^{[14]}, 7^{[34]}$ \\
11 & $0^{[1]}, 1^{[2]}, 2^{[2]}, 3^{[2]}, 4^{[10]}, 5^{[2]}, 6^{[22]}, 7^{[22]}, 8^{[22]}, 9^{[27]}$ \\
\hline
\end{tabular}

As noted by Laurent Bartholdi and Olivier Siegenthaler, there is a pattern in the ranks of the Dwyer quotients $M_{c}\left(\Gamma_{d}\right)$. For example, it may holds that

$$
M_{c}\left(\Gamma_{5}\right) \cong\left\{\begin{array}{cl}
0, & \text { if } c=0 \\
\mathbb{Z}_{5}^{2 m+1}, & \text { if } c \in\left\{2+\frac{3}{2}\left(5^{m}-1\right), \ldots, 1+\frac{3}{2}\left(5^{m}-1\right)+4 \cdot 5^{m}\right\} \\
\mathbb{Z}_{5}^{2 m+2}, & \text { if } c \in\left\{2+\frac{3}{2}\left(5^{m}-1\right)+4 \cdot 5^{m}, \ldots, 1+\frac{3}{2}\left(5^{m+1}-1\right)\right\}
\end{array}\right\}
$$

for $m \in \mathbb{N}_{0}$. This suggests the following conjecture.

Conjecture $\mathbf{F}$ Let $d$ be a prime. Then the Schur multiplier of $\Gamma_{d}$, modulo the Dwyerkernel, is infinitely generated d-elementary abelian. 
Finally, we summarize our results for $M_{c}\left(\Gamma_{d}\right)$ for $d \in\{4,8,9\}$. The abelian invariants of the Dwyer quotients $M_{c}\left(\Gamma_{d}\right)$ are as follows.

\begin{tabular}{|c|c|}
\hline$d$ & $M_{c}\left(\Gamma_{d}\right)$ \\
\hline 4 & $\begin{array}{r}(1)^{[1]}(2)^{[1]}(2,2)^{[1]}(2,4)^{[4]}(2,2,2,4)^{[1]} \\
(2,2,2,2,4)^{[4]}(2,2,2,4,4)^{[16]}(2,2,2,2,4,4)^{[1]}(2,2,2,2,2,4,4)^{[3]} \\
(2,2,2,2,2,2,4,4)^{[16]}(2,2,2,2,2,4,4,4)^{[64]}(2,2,2,2,2,2,4,4,4)^{[5]} \\
(2,2,2,2,2,2,2,4,4,4)^{[11]}(2,2,2,2,2,2,2,2,4,4,4)^{[26]}\end{array}$ \\
\hline 8 & $\begin{array}{r}(1)^{[1]}(8)^{[2]}(4,8)^{[3]}(2,4,8)^{[4]}(2,8,8)^{[1]}(2,2,8,8)^{[2]} \\
\left.(2,2,2,8,8)^{[2]}(2,2,4,8,8)^{[2]}(2,4,4,8,8)^{[2]}(2,4,8,8,8)^{[2]}\right] \\
(2,8,8,8,8)^{[8]}(2,2,8,8,8,8)^{[4]}(2,4,8,8,8,8)^{[20]}(2,2,4,8,8,8,8)^{[32]} \\
(2,2,8,8,8,8,8)^{[7]}(2,2,2,8,8,8,8,8)^{[16]}(2,2,2,2,8,8,8,8,8)^{[16]} \\
(2,2,2,4,8,8,8,8,8)^{[16]}(2,2,4,4,8,8,8,8,8)^{[3]}\end{array}$ \\
\hline 9 & $\begin{array}{r}(1)^{[1]}(9)^{[2]}(3,9)^{[2]}(3,3,9)^{[4]}(3,9,9)^{[2]} \\
(9,9,9)^{[2]}(3,9,9,9)^{[2]}(3,3,9,9,9)^{[4]}(3,9,9,9,9)^{[2]} \\
(9,9,9,9,9)^{[12]}(3,9,9,9,9,9)^{[18]}(3,3,9,9,9,9,9)^{[36]} \\
(3,9,9,9,9,9,9)^{[18]}(9,9,9,9,9,9,9)^{[17]}(3,9,9,9,9,9,9,9)^{[12]}\end{array}$ \\
\hline
\end{tabular}

Again, these computational results suggest that the groups $\Gamma_{d}$ are not finitely presentable. Further, the exponent of $M_{c}\left(\Gamma_{d}\right)$ is most likely the index $d$ itself.

\section{Acknowledgments}

I would like to thank Bettina Eick, Laurent Bartholdi, and Olivier Siegenthaler for many helpful discussions. I am grateful to the referee for valuable comments and suggestions.

René Hartung, Institute of Computational Mathematics, University of Braunschweig, Pockelsstrasse 14, 38106 Braunschweig, Germany

Email: $\quad$ r.hartung@tu-bs.de

June 2009 (accepted April 2010). 


\section{Bibliography}

[1] S. I. Adyan. Algorithmic unsolvability of problems of recognition of certain properties of groups. Dokl. Akad. Nauk SSSR (N.S.), 103:533-535, 1955.

[2] S. I. Adyan. Finitely presented groups and algorithms. Dokl. Akad. Nauk SSSR (N.S.), 117:9-12, 1957.

[3] S. I. Adyan. Unsolvability of some algorithmic problems in the theory of groups. Trudy Moskov. Mat. Obšč., 6:231-298, 1957.

[4] S. I. Adyan and V. G. Durnev. Algorithmic problems for groups and semigroups. Uspekhi Mat. Nauk, 55(2(332)):3-94, 2000.

[5] S. V. Alešin. Finite automata and the Burnside problem for periodic groups. Mat. Zametki, 11:319-328, 1972.

[6] L. Bartholdi. Endomorphic presentations of branch groups. J. Algebra, 268(2):419443, 2003.

[7] L. Bartholdi. Lie algebras and growth in branch groups. Pacific J. Math., 218(2):241-282, 2005.

[8] L. Bartholdi. FR - Computations with functionally recursive groups, 2009. A refereed GAP 4 package, see [50].

[9] L. Bartholdi, B. Eick, and R. Hartung. A nilpotent quotient algorithm for certain infinitely presented groups and its applications. Internat. J. Algebra Comput., 18(8):1321-1344, 2008.

[10] L. Bartholdi and R. I. Grigorchuk. Lie methods in growth of groups and groups of finite width. In Computational and geometric aspects of modern algebra (Edinburgh, 1998), volume 275 of London Math. Soc. Lecture Note Ser., pages 1-27. Cambridge Univ. Press, Cambridge, 2000.

[11] L. Bartholdi and R. I. Grigorchuk. On parabolic subgroups and Hecke algebras of some fractal groups. Serdica Math. J., 28(1):47-90, 2002. 
[12] L. Bartholdi, R. I. Grigorchuk, and Z. Šuniḱ. Branch groups. In Handbook of algebra, Vol. 3, pages 989-1112. North-Holland, Amsterdam, 2003.

[13] L. Bartholdi and F. Pochon. On growth and torsion of groups. Groups Geom. Dyn., 3(4):525-539, 2009.

[14] L. Bartholdi and O. Siegenthaler. The twisted twin of the Grigorchuk group. Internat. J. Algebra Comput., 24(4):465-488, 2010.

[15] L. Bartholdi, O. Siegenthaler, and P. Zalesskii. The congruence subgroup problem for branch groups. Preprint, 2010.

[16] L. Bartholdi and B. Virág. Amenability via random walks. Duke Math. J., 130(1):39-56, 2005.

[17] G. Baumslag. Wreath products and finitely presented groups. Math. Z., 75:22-28, $1960 / 1961$.

[18] G. Baumslag. A finitely generated, infinitely related group with trivial multiplicator. Bull. Austral. Math. Soc., 5:131-136, 1971.

[19] G. Baumslag. Topics in combinatorial group theory. Lectures in Mathematics ETH Zürich. Birkhäuser Verlag, Basel, 1993.

[20] G. Baumslag, W. W. Boone, and B. H. Neumann. Some unsolvable problems about elements and subgroups of groups. Math. Scand., 7:191-201, 1959.

[21] G. Baumslag and D. Solitar. Some two-generator one-relator non-Hopfian groups. Bull. Amer. Math. Soc., 68:199-201, 1962.

[22] M. Benli. Indicable groups and endomorphic presentations. Glasgow Math. J., 2011. Available on CJO 2011 doi:10.1017/S0017089511000632.

[23] M. Bezem and J. W. Klop. Abstract reduction systems. In Term rewriting systems, volume 55 of Cambridge Tracts Theoret. Comput. Sci., pages 7-23. Cambridge Univ. Press, Cambridge, 2003.

[24] N. Blackburn and L. Evens. Schur multipliers of p-groups. J. Reine Angew. Math., 309:100-113, 1979.

[25] V. V. Bludov. On residually torsion-free nilpotent groups. J. Group Theory, 12(4):579-590, 2009.

[26] O. Bogopolski and E. Ventura. A recursive presentation for Mihailova's subgroup. Groups Geom. Dyn., 4(3):407-417, 2010.

[27] W. W. Boone. The word problem. Ann. of Math. (2), 70:207-265, 1959. 
[28] W. Bosma, J. Cannon, and C. Playoust. The Magma algebra system. I. The user language. J. Symbolic Comput., 24(3-4):235-265, 1997. Computational algebra and number theory (London, 1993).

[29] A. M. Brunner, S. Sidki, and A. C. Vieira. A just nonsolvable torsion-free group defined on the binary tree. J. Algebra, 211(1):99-114, 1999.

[30] W. Burnside. On unsettled question in the theory of discontinuous groups. Quart. J. Pure Appl. Math., 33:230-238, 1902.

[31] C. M. Campbell, E. F. Robertson, N. Ruškuc, and R. M. Thomas. ReidemeisterSchreier type rewriting for semigroups. Semigroup Forum, 51(1):47-62, 1995.

[32] J. J. Cannon, L. A. Dimino, G. Havas, and J. M. Watson. Implementation and analysis of the Todd-Coxeter algorithm. Math. Comp., 27:463-490, 1973.

[33] T. Ceccherini-Silberstein, F. Scarabotti, and F. Tolli. The top of the lattice of normal subgroups of the Grigorchuk group. J. Algebra, 246(1):292-310, 2001.

[34] C. R. J. Clapham. An embedding theorem for finitely generated groups. Proc. London Math. Soc. (3), 17:419-430, 1967.

[35] M. Conder and P. Dobcsányi. Applications and adaptations of the low index subgroups procedure. Math. Comp., 74(249):485-497 (electronic), 2005.

[36] H. S. M. Coxeter and W. O. J. Moser. Generators and relations for discrete groups. Second edition. Ergebnisse der Mathematik und ihrer Grenzgebiete, Neue Folge, Band 14. Springer-Verlag, Berlin, 1965.

[37] M. M. Day. Amenable semigroups. Illinois J. Math., 1:509-544, 1957.

[38] P. de la Harpe. Topics in geometric group theory. Chicago Lectures in Mathematics. University of Chicago Press, Chicago, IL, 2000.

[39] M. Dehn. Über unendliche diskontinuierliche Gruppen. Math. Ann., 71(1):116-144, 1911.

[40] A. Dietze and M. Schaps. Determining subgroups of a given finite index in a finitely presented group. Canad. J. Math., 26:769-782, 1974.

[41] P. Dobcsányi. LowX - Version 0.11, 2007. Available as http://web.cs.dal.ca/ peter/software/lowx/lowx-0.11.tar.gz.

[42] B. Eick and R. Hartung. Investigating some self-similar groups via nilpotent quotients. Unpublished manuscript. Accepted for the proceedings of Groups St Andrews 2009, 2010. 
[43] B. Eick and M. Horn. Computing polycyclic quotients of finitely (L-)presented groups via Groebner bases. In Proceedings of the Third international congress conference on Mathematical software, ICMS'10, pages 50-53, Berlin, Heidelberg, 2010. Springer-Verlag.

[44] B. Eick and W. Nickel. Computing the Schur multiplicator and the nonabelian tensor square of a polycyclic group. J. Algebra, 320(2):927-944, 2008.

[45] J. Fabrykowski and N. Gupta. On groups with sub-exponential growth functions. J. Indian Math. Soc. (N.S.), 49(3-4):249-256 (1987), 1985.

[46] J. Fabrykowski and N. Gupta. On groups with sub-exponential growth functions. II. J. Indian Math. Soc. (N.S.), 56(1-4):217-228, 1991.

[47] W. Feit and J. G. Thompson. Solvability of groups of odd order. Pacific J. Math., 13:775-1029, 1963.

[48] H. Felsch. Programmierung der Restklassenabzählung einer Gruppe nach Untergruppen. Numer. Math., 3:250-256, 1961.

[49] G. A. Fernández-Alcober and A. Zugadi-Reizabal. GGS-groups: order of congruence quotients and Hausdorff dimension. Preprint. Available as arxiv.org/abs/1108.2289, 2011.

[50] The GAP Group. GAP - Groups, Algorithms, and Programming, Version 4.4.10, 2007.

[51] E. S. Golod. On nil-algebras and finitely approximable p-groups. Izv. Akad. Nauk SSSR Ser. Mat., 28:273-276, 1964.

[52] C. M. Gordon. Some embedding theorems and undecidability questions for groups. In Combinatorial and geometric group theory (Edinburgh, 1993), volume 204 of London Math. Soc. Lecture Note Ser., pages 105-110. Cambridge Univ. Press, Cambridge, 1995.

[53] R. I. Grigorchuk. On Burnside's problem on periodic groups. Funktsional. Anal. $i$ Prilozhen., 14(1):53-54, 1980.

[54] R. I. Grigorchuk. On the Milnor problem of group growth. Dokl. Akad. Nauk SSSR, 271(1):30-33, 1983.

[55] R. I. Grigorchuk. An example of a finitely presented amenable group that does not belong to the class EG. Mat. Sb., 189(1):79-100, 1998.

[56] R. I. Grigorchuk. On the system of defining relations and the Schur multiplier of periodic groups generated by finite automata. In Groups St. Andrews 1997 in Bath, I, volume 260 of London Math. Soc. Lecture Note Ser., pages 290-317. Cambridge Univ. Press, Cambridge, 1999. 
[57] R. I. Grigorchuk. Just infinite branch groups. In New horizons in pro-p groups, volume 184 of Progr. Math., pages 121-179. Birkhäuser Boston, Boston, MA, 2000.

[58] R. I. Grigorchuk. Solved and unsolved problems around one group. In Infinite groups: geometric, combinatorial and dynamical aspects, volume 248 of Progr. Math., pages 117-218. Birkhäuser, Basel, 2005.

[59] R. I. Grigorchuk and Z. Šunik. Asymptotic aspects of Schreier graphs and Hanoi Towers groups. C. R. Math. Acad. Sci. Paris, 342(8):545-550, 2006.

[60] R. I. Grigorchuk and A. Żuk. On a torsion-free weakly branch group defined by a three state automaton. Internat. J. Algebra Comput., 12(1-2):223-246, 2002. International Conference on Geometric and Combinatorial Methods in Group Theory and Semigroup Theory (Lincoln, NE, 2000).

[61] R. I. Grigorchuk and A. Żuk. Spectral properties of a torsion-free weakly branch group defined by a three state automaton. In Computational and statistical group theory (Las Vegas, NV/Hoboken, NJ, 2001), volume 298 of Contemp. Math., pages 57-82. Amer. Math. Soc., Providence, RI, 2002.

[62] N. Gupta and S. Sidki. On the Burnside problem for periodic groups. Math. Z., 182(3):385-388, 1983.

[63] R. Hartung. An infinite polycyclic quotient algorithm for finitely $L$-presented groups. Unpublished manuscript, 2008.

[64] R. Hartung. A nilpotent quotient algorithm for finitely L-presented groups. Diploma thesis, Technische Universität Braunschweig, 2008.

[65] R. Hartung. NQL - Nilpotent quotients of L-presented groups, 2009. A refereed GAP 4 package, see [50].

[66] R. Hartung. Approximating the Schur multiplier of certain infinitely presented groups via nilpotent quotients. LMS J. Comput. Math., 13:260-271, 2010. Preprint available as arxiv.org/abs/1106.1098.

[67] R. Hartung. Coset enumeration for certain infinitely presented groups. Internat. J. Algebra Comput., 21(8):1369-1380, 2011. Preprint available as arxiv.org/abs/1106.0259.

[68] R. Hartung. Investigating self-similar groups using their finite $L$-presentation. Preprint available as arxiv.org/abs/1204.4279, 2012.

[69] R. Hartung. A note on invariantly finitely $L$-presented groups. Preprint available as arxiv.org/abs/1205.0143, 2012.

[70] R. Hartung. A Reidemeister-Schreier theorem for finitely $L$-presented groups. Internat. Elec. J. Algebra, 11:125-159, 2012. Preprint available as arxiv.org/abs/1108.2403. 
[71] R. Hartung and G. Traustason. Refined solvable presentations for polycyclic groups. International Journal of Group Theory, 1(2):1-17, 2012. Preprint available as arxiv.org/abs/1102.1650.

[72] G. Havas. A Reidemeister-Schreier program. In Proceedings of the Second International Conference on the Theory of Groups (Australian Nat. Univ., Canberra, 1973), pages 347-356. Lecture Notes in Math., Vol. 372, Berlin, 1974. Springer.

[73] G. Havas, P. E. Kenne, J. S. Richardson, and E. F. Robertson. A Tietze transformation program. In Computational group theory (Durham, 1982), pages 69-73. Academic Press, London, 1984.

[74] G. Havas and M. F. Newman. Application of computers to questions like those of Burnside. In Burnside groups (Proc. Workshop, Univ. Bielefeld, Bielefeld, 1977), volume 806 of Lecture Notes in Math., pages 211-230. Springer, Berlin, 1980.

[75] G. Havas and C. Ramsay. ACE - Version 3, 1999. Available as http://www.csee.uq.edu.au/ havas/ace3.tar.gz.

[76] G. Havas and C. Ramsay. Proving a group trivial made easy: a case study in coset enumeration. Bull. Austral. Math. Soc., 62(1):105-118, 2000.

[77] G. Havas and E. F. Robertson. Application of computational tools for finitely presented groups. In Computational support for discrete mathematics (Piscataway, NJ, 1992), volume 15 of DIMACS Ser. Discrete Math. Theoret. Comput. Sci., pages 29-39. Amer. Math. Soc., Providence, RI, 1994.

[78] G. Havas and E. F. Robertson. Central factors of deficiency zero groups. Comm. Algebra, 24(11):3483-3487, 1996.

[79] G. Havas and E. F. Robertson. The $F^{a, b, c}$ conjecture. I. Irish Math. Soc. Bull., (56):75-80, 2005.

[80] G. Havas, E. F. Robertson, and D. C. Sutherland. The $F^{a, b, c}$ conjecture is true. II. J. Algebra, 300(1):57-72, 2006.

[81] G. Higman. Subgroups of finitely presented groups. Proc. Roy. Soc. Ser. A, 262:455-475, 1961.

[82] D. F. Holt. The calculation of the Schur multiplier of a permutation group. In Computational group theory (Durham, 1982), pages 307-319. Academic Press, London, 1984 .

[83] D. F. Holt and S. Rees. The isom-quotpic package, 1997.

[84] G. Huet. Confluent reductions: abstract properties and applications to term rewriting systems. J. Assoc. Comput. Mach., 27(4):797-821, 1980. 
[85] I. Kapovich and D. T. Wise. On the failure of the co-Hopf property for subgroups of word-hyperbolic groups. Israel J. Math., 122:125-147, 2001.

[86] D. E. Knuth and P. B. Bendix. Simple word problems in universal algebras. In Computational Problems in Abstract Algebra (Proc. Conf., Oxford, 1967), pages 263-297. Pergamon, Oxford, 1970.

[87] W. Krull. Über verallgemeinerte endliche Abelsche Gruppen. M. Z., 23:161-196, 1925.

[88] J. Leech. Coset enumeration on digital computers. Proc. Camb. Philos. Soc., $59: 257-267,1963$.

[89] C. R. Leedham-Green. A soluble group algorithm. In Computational group theory (Durham, 1982), pages 85-101. Academic Press, London, 1984.

[90] C. R. Leedham-Green, C. E. Praeger, and L. H. Soicher. Computing with group homomorphisms. J. Symbolic Comput., 12(4-5):527-532, 1991. Computational group theory, Part 2.

[91] A. Lindenmayer. Cellular automata, formal languages and developmental systems. In Logic, methodology and philosophy of science, IV (Proc. Fourth Internat. Congr., Bucharest, 1971), pages 677-691. Studies in Logic and Foundations of Math., Vol. 74. North-Holland, Amsterdam, 1973.

[92] E. H. Lo. A polycyclic quotient algorithm. In Groups and computation, II (New Brunswick, NJ, 1995), volume 28 of DIMACS Ser. Discrete Math. Theoret. Comput. Sci., pages 159-167. Amer. Math. Soc., Providence, RI, 1997.

[93] E. H. Lo. A polycyclic quotient algorithm. J. Symbolic Comput., 25(1):61-97, 1998.

[94] J. Lockhart. Decision problems in classes of group presentations with uniformly solvable word problem. Arch. Math. (Basel), 37(1):1-6, 1981.

[95] R. C. Lyndon and P. E. Schupp. Combinatorial group theory. Springer-Verlag, Berlin, 1977. Ergebnisse der Mathematik und ihrer Grenzgebiete, Band 89.

[96] I. G. Lysënok. A set of defining relations for the Grigorchuk group. Mat. Zametki, 38(4):503-516, 634, 1985.

[97] W. Magnus, A. Karrass, and D. Solitar. Combinatorial group theory: Presentations of groups in terms of generators and relations. Interscience Publishers [John Wiley \& Sons, Inc.], New York-London-Sydney, 1966.

[98] R. V. Mikhaĭlov. Residual nilpotence and residual solvability of groups. Mat. Sb., 196(11):109-126, 2005.

[99] C. F. Miller, III. Decision problems for groups - survey and reflections. In $\mathrm{Al}$ gorithms and classification in combinatorial group theory (Berkeley, CA, 1989), volume 23 of Math. Sci. Res. Inst. Publ., pages 1-59. Springer, New York, 1992. 
[100] J. Milnor. A note on curvature and fundamental group. J. Differential Geometry, $2: 1-7,1968$.

[101] V. Nekrashevych. Self-similar groups, volume 117 of Mathematical Surveys and Monographs. American Mathematical Society, Providence, RI, 2005.

[102] J. Neubüser. An elementary introduction to coset table methods in computational group theory. In Groups-St. Andrews 1981 (St. Andrews, 1981), volume 71 of London Math. Soc. Lecture Note Ser., pages 1-45. Cambridge Univ. Press, Cambridge, 1982 .

[103] B. H. Neumann. Some remarks on infinite groups. J. Lond. Math. Soc., 12:120-127, 1937.

[104] B. H. Neumann. Proofs. Math. Intelligencer, 2(1):17-24, 1979/80.

[105] M. F. Newman and E. A. O'Brien. Application of computers to questions like those of Burnside. II. Internat. J. Algebra Comput., 6(5):593-605, 1996.

[106] M. H. A. Newman. On theories with a combinatorial definition of "equivalence.". Ann. of Math. (2), 43:223-243, 1942.

[107] W. Nickel. Computing nilpotent quotients of finitely presented groups. In $G e-$ ometric and computational perspectives on infinite groups (Minneapolis, $M N$ and New Brunswick, NJ, 1994), volume 25 of DIMACS Ser. Discrete Math. Theoret. Comput. Sci., pages 175-191. Amer. Math. Soc., Providence, RI, 1996.

[108] A. C. Niemeyer. ANUSQ - Soluble quotient algorithm for finitely presented groups, 1994. A GAP3 package, see [50].

[109] A. C. Niemeyer. A finite soluble quotient algorithm. J. Symbolic Comput., 18(6):541-561, 1994.

[110] A. C. Niemeyer. Computing finite soluble quotients. In Computational algebra and number theory (Sydney, 1992), volume 325 of Math. Appl., pages 75-82. Kluwer Acad. Publ., Dordrecht, 1995.

[111] E. Noether. Hyperkomplexe Größen und Darstellungstheorie. M. Z., 30:641-692, 1929.

[112] P. S. Novikov. Unsolvability of the conjugacy problem in the theory of groups. Izv. Akad. Nauk SSSR. Ser. Mat., 18:485-524, 1954.

[113] P. S. Novikov. Ob algoritmičeskoŭ nerazrešimosti problemy toždestva slov v teorii grupp. Trudy Mat. Inst. im. Steklov. no. 44. Izdat. Akad. Nauk SSSR, Moscow, 1955 .

[114] A. Y. Ol'shanskii and M. V. Sapir. Non-amenable finitely presented torsion-bycyclic groups. Electron. Res. Announc. Amer. Math. Soc., 7:63-71 (electronic), 2001. 
[115] S. M. H. W. Perlo-Freeman. Infinite Systems of Reductions and Knuth-Bendix Orderings. Dissertation, University of Warwick, 1996.

[116] E. L. Pervova. Everywhere dense subgroups of a group of tree automorphisms. Tr. Mat. Inst. Steklova, 231(Din. Sist., Avtom. i Beskon. Gruppy):356-367, 2000.

[117] W. Plesken. Towards a soluble quotient algorithm. J. Symbolic Comput., 4(1):111$122,1987$.

[118] M. O. Rabin. Recursive unsolvability of group theoretic problems. Ann. of Math. (2), 67:172-194, 1958.

[119] K. Reidemeister. Knoten und Gruppen. Abhandlungen Hamburg, 5:7-23, 1926.

[120] E. F. Robertson. Tietze transformations with weighted substring search. J. Symbolic Comput., 6(1):59-64, 1988.

[121] D. J. S. Robinson. A course in the theory of groups, volume 80 of Graduate Texts in Mathematics. Springer-Verlag, New York, second edition, 1996.

[122] A. V. Rozhkov. Centralizers of elements in a group of tree automorphisms. Izv. Ross. Akad. Nauk Ser. Mat., 57(6):82-105, 1993.

[123] A. V. Rozhkov. Lower central series of a group of tree automorphisms. Mat. Zametki, 60(2):225-237, 319, 1996.

[124] O. Schreier. Die Untergruppen der freien Gruppen. Abhandlungen Hamburg, 5:161$183,1927$.

[125] D. Segal. Polycyclic groups, volume 82 of Cambridge Tracts in Mathematics. Cambridge University Press, Cambridge, 1983.

[126] D. Segal. Decidable properties of polycyclic groups. Proc. London Math. Soc. (3), 61(3):497-528, 1990.

[127] V. Sergiescu. A quick introduction to Burnside's problem. In Group theory from a geometrical viewpoint (Trieste, 1990), pages 622-629. World Sci. Publ., River Edge, NJ, 1991.

[128] S. Sidki. On a 2-generated infinite 3-group: the presentation problem. J. Algebra, 110(1):13-23, 1987.

[129] C. C. Sims. Computation with finitely presented groups, volume 48 of Encyclopedia of Mathematics and its Applications. Cambridge University Press, Cambridge, 1994.

[130] C. C. Sims. The Knuth-Bendix procedure for strings and large rewriting systems. In Computational and statistical group theory (Las Vegas, NV/Hoboken, NJ, 2001), volume 298 of Contemp. Math., pages 115-124. Amer. Math. Soc., Providence, RI, 2002 . 
[131] Z. Šunić. Hausdorff dimension in a family of self-similar groups. Geom. Dedicata, 124:213-236, 2007.

[132] H. Tietze. Über die topologischen Invarianten mehrdimensionaler Mannigfaltigkeiten. Monatsh. Math. Phys., 19(1):1-118, 1908.

[133] J. Todd and H. Coxeter. A practical method for enumerating cosets of a finite abstract group. Proceedings of the Edinburgh Mathematical Society (Series 2), 5(1):26-34, 1936.

[134] A. C. Vieira. On the lower central series and the derived series of the Gupta-Sidki 3-group. Comm. Algebra, 26(4):1319-1333, 1998.

[135] J. W. Wamsley. Computing soluble groups. In Group theory (Proc. Miniconf., Australian Nat. Univ., Canberra, 1975), pages 118-125. Lecture Notes in Math., Vol. 573. Springer, Berlin, 1977. 


\section{Curriculum Vitæ}

\section{René Hartung}

Title:

Diplom Mathematiker (Dipl.-Math.)

Date of birth: September 8, 1981

Nationality: German

Address: $\quad$ Georg-August Universität zu Göttingen, Bunsenstraße 3-5, 37073 Göttingen, Germany

\section{Education}

07/2008 Diplom in Mathematik, Technische Universität Braunschweig Thesis: A nilpotent quotient algorithm for finitely L-presented groups

04/2004 Vordiplom in Mathematik, Technische Universität Braunschweig

10/2003 Vordiplom in Physik, Technische Universität Braunschweig

07/2000 Abitur, Werner-von-Siemens Landesgymnasium, Magdeburg

\section{Publications}

(1) A Reidemeister Schreier theorem for finitely $L$-presented groups. Int. Electron. J. Algebra. 11 (2012), p. 125-159.

Preprint available as arxiv.org/abs/1108.2403.

(2) Coset enumeration for certain infinitely presented groups. Internat. J. Algebra Comput. 21 (2011), no. 8., p. 1369-1380.

DOI 10.1142/S0218196711006637. Preprint available as arxiv.org/abs/1106.0259.

(3) Approximating the Schur multiplier of certain infinitely presented groups via nilpotent quotients. LMS J. Comput. Math. 13 (2010), p. 260-271. DOI 10.1112/S1461157009000229. Preprint available as arxiv.org/abs/1106. 1098.

(4) A nilpotent quotient algorithm for certain infinitely presented groups and its applications. With L. Bartholdi and B. Eick. Internat. J. Algebra Comput. 18 (2008), no. 8, p. 1321-1344.

DOI 10.1142/S0218196708004871. Preprint available as arxiv .org/abs/0706. 3131.

(5) Refined solvable presentations for polycyclic groups. With G. Traustason. International Journal of Group Theory, 1(2):1-17, 2012.

Preprint available as arxiv.org/abs/1102.1650. 